\title{
Modernization of Technical Requirements for Licensing of Advanced Non-Light Water Reactors: Selection and Evaluation of Licensing Basis Events
}

Wayne L Moe, Amir Afzali

March 2020

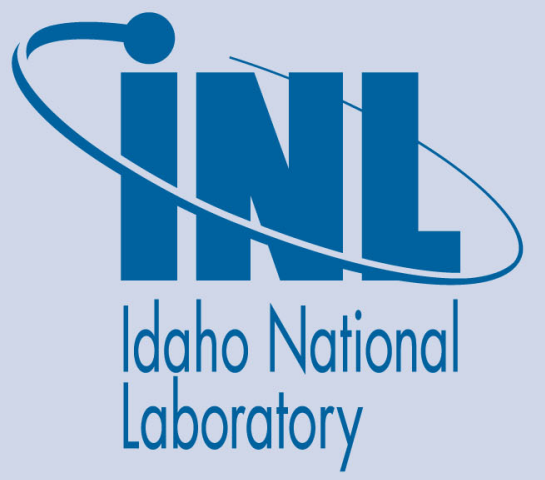

The INL is a U.S. Department of Energy National Laboratory operated by Battelle Energy Alliance 


\section{Modernization of Technical Requirements for Licensing of Advanced Non-Light Water Reactors: Selection and Evaluation of Licensing Basis Events}

Wayne L Moe, Amir Afzali

March 2020

Idaho National Laboratory Idaho Falls, Idaho 83415

http://www.inl.gov

Prepared for the

U.S. Department of Energy

Under DOE Idaho Operations Office

Contract DE-AC07-05ID14517 


\section{$\Delta$ \\ Southern Company}

Modernization of Technical Requirements

for Licensing of Advanced Non-Light Water Reactors:

Selection and Evaluation of Licensing Basis Events

Document Number

SC-29980-100 Rev 1

March 2020

Prepared for:

U.S. Department of Energy (DOE)

Office of Nuclear Energy

Under DOE Idaho Operations Office

Contract DE-AC07-05ID14517 


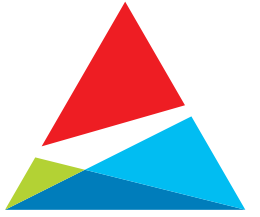 \\ Southern Company}

Modernization of Technical Requirements

for Licensing of Advanced Non-Light Water Reactors:

Selection and Evaluation of Licensing Basis Events

Document Number

SC-29980-100 Rev 1

Issued by:

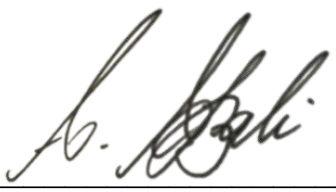

Amir Afzali, Next Generation Licensing and Policy Director Southern Company Services

Date 


\section{Disclaimer}

This report was prepared as an account of work sponsored by an agency of the United States (U.S.) Government. Neither the U.S. Government nor any agency thereof, nor any of their employees, nor Southern Company, Inc., nor any of its employees, nor any of its subcontractors, nor any of its sponsors or co-funders, makes any warranty, expressed or implied, or assumes any legal liability or responsibility for the accuracy, completeness, or usefulness of any information, apparatus, product, or process disclosed, or represents that its use would not infringe privately owned rights. Reference herein to any specific commercial product, process, or service by trade name, trademark, manufacturer or otherwise, does not necessarily constitute or imply its endorsement, recommendation, or favoring by the U.S. Government or any agency thereof. The views and opinions of authors expressed herein do not necessarily state or reflect those of the U.S. Government or any agency thereof. 


\begin{abstract}
This report, "Modernization of Technical Requirements for Licensing of Advanced Non-Light Water Reactors: Selection and Evaluation of Licensing Basis Events," represents a key element in the development of a methodology for the efficient licensing of advanced non-light water reactors (non-LWRs). It is the result of a Licensing Modernization Project (LMP) led by Southern Company and cost-shared by the U.S. Department of Energy (DOE). The LMP will result in detailed proposals for establishing licensing technical requirements to facilitate riskinformed and performance-based design and licensing of advanced non-LWRs. Such a methodology acknowledges enhancements in safety achievable with advanced designs and reflects more recent states of knowledge regarding safety and design innovation, creating an opportunity for reduced regulatory complexity with increased levels of safety. The project builds on best practices as well as previous activities through DOE and industry-sponsored advanced reactor licensing initiatives.
\end{abstract}

The LMP objective is to assist the Nuclear Regulatory Commission (NRC) in developing regulatory guidance for licensing advanced non-LWR plants.

This report presents a modern, technology-inclusive, risk-informed, and performance-based (TIRIPB) approach to identifying Licensing Basis Events (LBEs), which cover a spectrum of events considered in the design and licensing of a nuclear power plant. A key licensing outcome of this approach is the structured selection of Design Basis Accidents (DBAs) that are traditionally analyzed in Chapter 15 of the license application.

Development of the LBE selection approach begins with a review of the relevant regulatory policy and available guidance for selecting LBEs. From this review, desirable attributes of an LBE selection and evaluation process are defined and used to develop the proposed approach. This report describes the methodology for selecting and evaluating LBEs and sets forth issues for resolution in order to facilitate an effective safety design approach leading to license applications for advanced non-LWRs.

This report builds on the development and subsequent NRC staff and Advisory Committee on Reactor Safeguards (ACRS) reviews of an LBE white paper for DOE's Next Generation Nuclear Plant (NGNP), a modular high-temperature gas-cooled reactor (mHTGR), which was derived from earlier precedents of the MHTGR (a specific prismatic mHTGR developed by DOE) and the Pebble Bed Modular Reactor. The proposed LBE method is intended for use with the full spectrum of advanced non-LWR concepts currently under consideration for development. The technology-inclusive capabilities of the proposed method are demonstrated using example LBEs from the MHTGR and the Power Reactor Innovative Small Module. The information in this report is intended to serve as the basis for interactions with the NRC staff leading to the development of regulatory guidance for the preparation of license applications. 


\section{Table of Contents}

Disclaimer iii

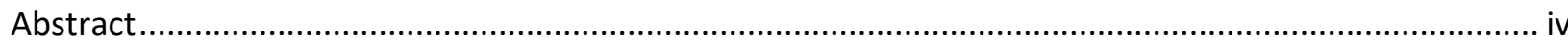

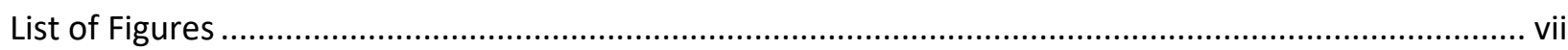

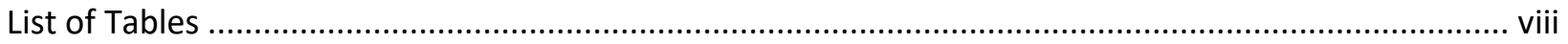

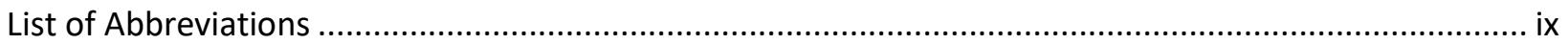

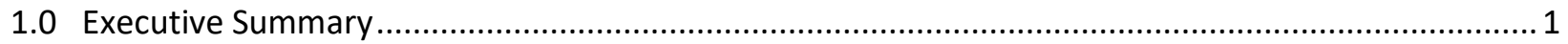

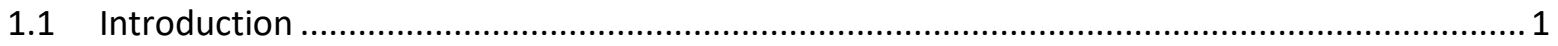

1.2 Definition of Licensing Basis Events ................................................................................. 1

1.3 LMP Approach to Selecting and Evaluating LBEs ............................................................. 2

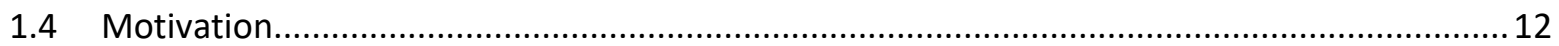

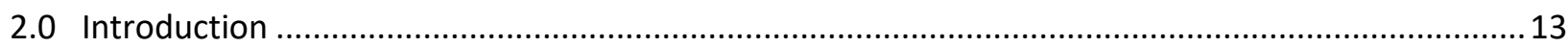

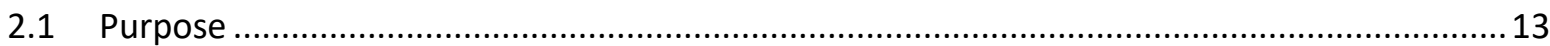

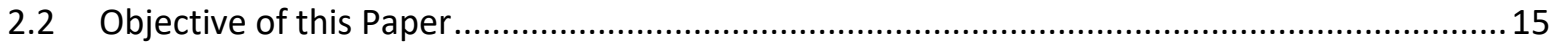

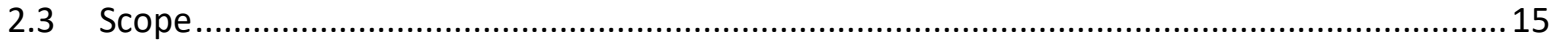

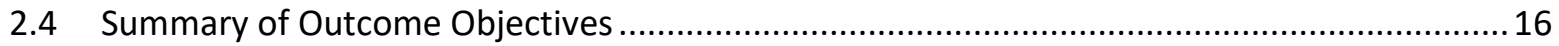

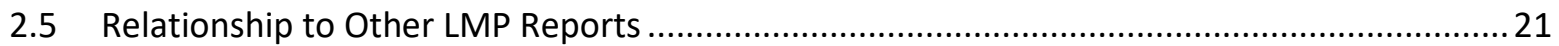

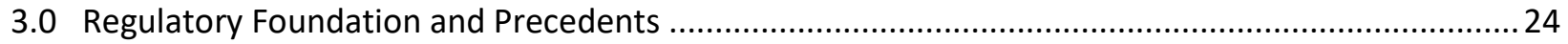

3.1 Regulatory Foundation and Precedent Review Summary ................................................. 24

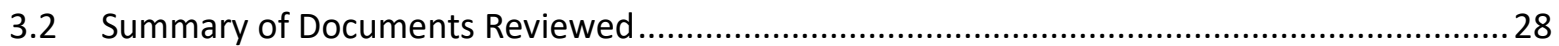

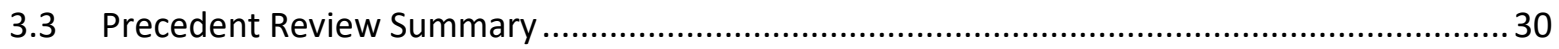

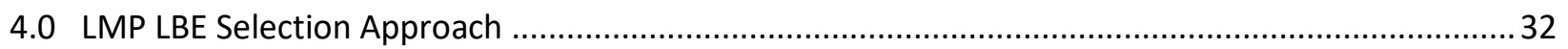

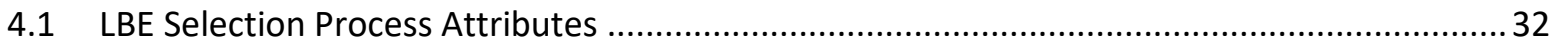

4.2 Review of Previous LBE Selection Approaches ….............................................................. 33

4.2.1 Interpretation of 10 CFR 20 and 10 CFR 50 Annual Exposure Limits.........................36

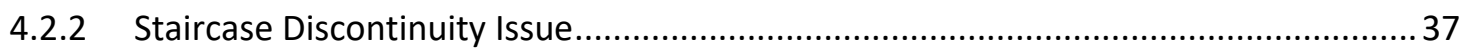

4.2.3 Plant-Year Versus Reactor-Year Frequency Basis ....................................................... 40

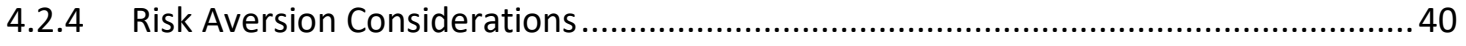

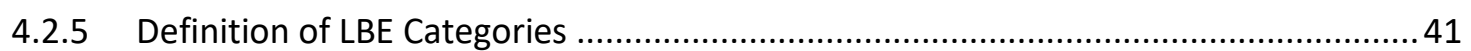

4.2.6 Risk Evaluation of LBEs and Integrated Risk Assessment .......................................... 42

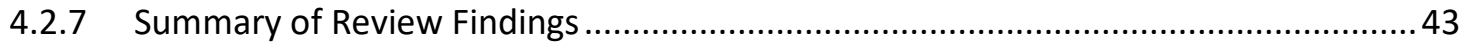

4.3 LMP Revisions to NGNP Frequency-Consequence Evaluation Criteria ................................... 44

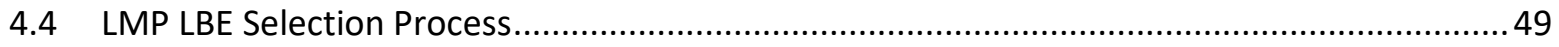




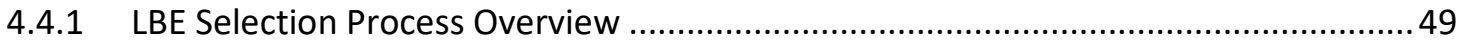

4.4.2 Evolution of LBEs Through Design and Licensing Stages .........................................58

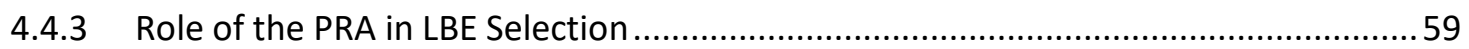

4.4.4 Structuring PRA Event Sequence Logic for LBE Selection ........................................ 62

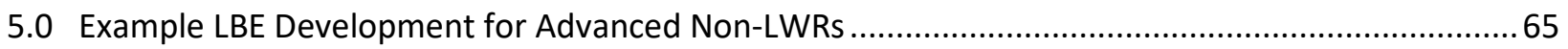

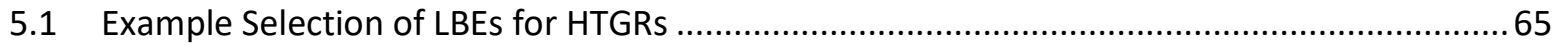

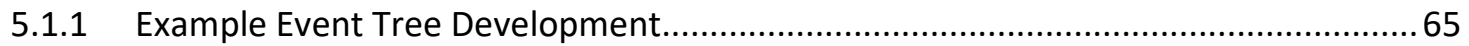

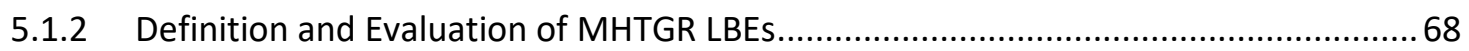

5.1.3 Definition of MHTGR DBAs for Chapter 15 Evaluation ........................................... 72

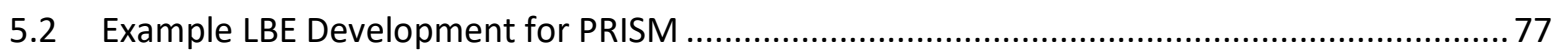

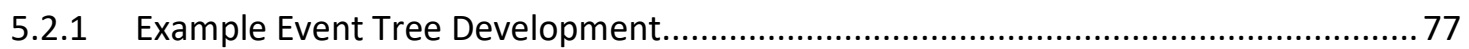

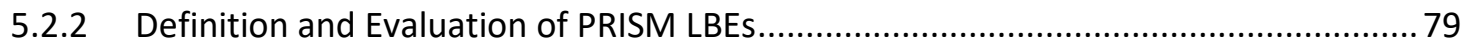

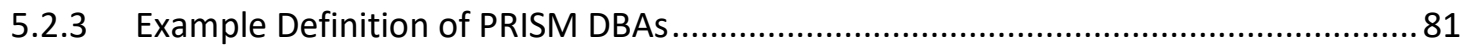

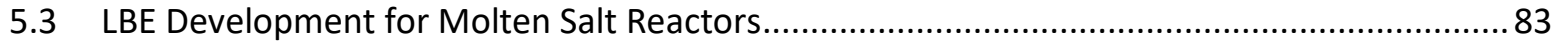

5.3.1 LBEs for Fluoride Salt-Cooled High Temperature Reactors .....................................83

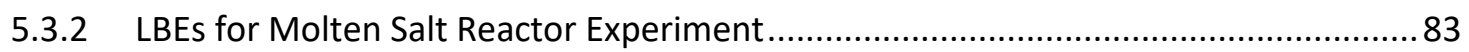

5.4 Example LBE Development in LMP Table Top Exercises .....................................................84

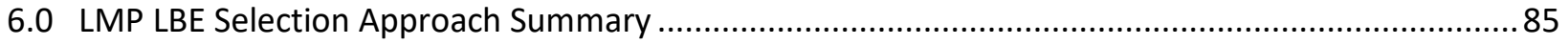

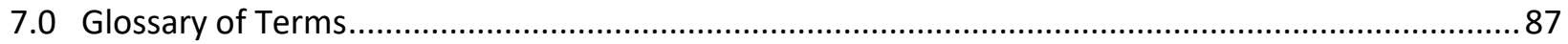

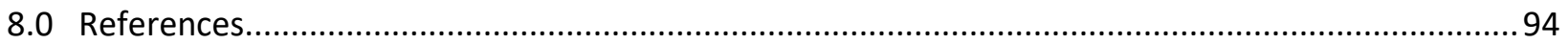

Appendix A-Regulatory Foundation and Precedents ..........................................................................

Appendix B-Technical Basis of the LMP Frequency-Consequence Target .......................................... B-1

Appendix C-LMP Documentation and Frequently Asked Questions ................................................... 


\section{List of Figures}

Figure 1-1. Process for Selecting and Evaluating Licensing Basis Events ................................................ 4

Figure 1-2. Frequency-Consequence Target Proposed for LMP …................................................. 8

Figure 2-1. Elements of TI-RIPB Licensing Modernization Methodology ................................................. 14

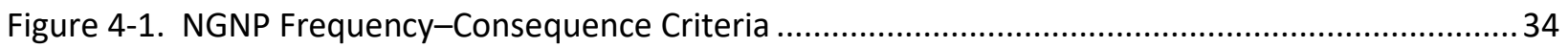

Figure 4-2. NUREG-1860 Frequency-Consequence Criteria............................................................... 35

Figure 4-3. Part of NGNP Frequency-Consequence Criteria Illustrating Staircase Issue ........................ 38

Figure 4-4. Frequency Versus Consequence Limit Line Proposed by Farmer ${ }^{[68]}$..................................... 41

Figure 4-5. Frequency-Consequence Evaluation Criteria Proposed for LMP ......................................... 46

Figure 4-6. Comparison of LMP and NGNP Frequency-Consequence Criteria .................................... 47

Figure 4-7. Comparison of LMP and NUREG-1860 Frequency-Consequence Criteria ............................. 48

Figure 4-8. Process for Selecting and Evaluating Licensing Basis Events .............................................50

Figure 4-9. Flow Chart for Initial PRA Model Development..................................................................60

Figure 5-1. Event Tree for MHTGR Very Small Leaks in Helium Pressure Boundary .................................66

Figure 5-2. Event Tree for MHTGR Loss of Offsite Power and Turbine Trip .........................................67

Figure 5-3. Event Tree for MHTGR Steam Generator Tube Rupture …...............................................68

Figure 5-4. Comparison of MHTGR LBE Frequencies and Consequences Frequency-Dose Criteria.......... 71

Figure 5-5. MHTGR Safety Functions Including Those Required to Meet 10 CFR 50.34 Limits ................. 73

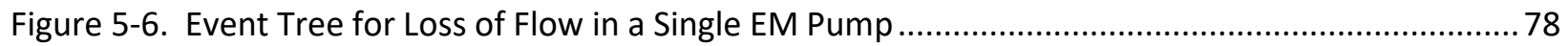

Figure 5-7. Comparison of PRISM LBE Frequencies and Consequences and LMP FrequencyConsequence Targets 


\section{List of Tables}

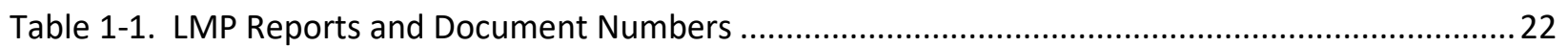

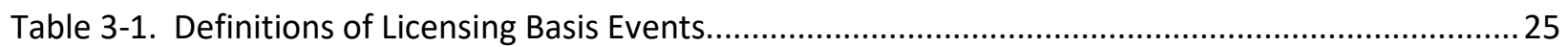

Table 3-2. Documents Reviewed for Regulatory Bases and Precedents ................................................28

Table 4-1. Examples of Required Safety Functions Identified in LMP Demonstrations...........................63

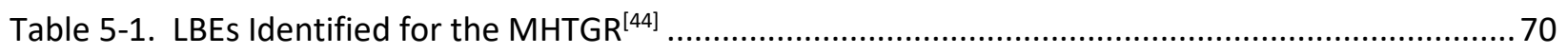

Table 5-2. Evaluation of Core Heat Removal SSCs for DBE-11 ............................................................ 73

Table 5-3. Evaluation of MHTGR SSCs for Core Heat Removal Safety Function ..................................... 74

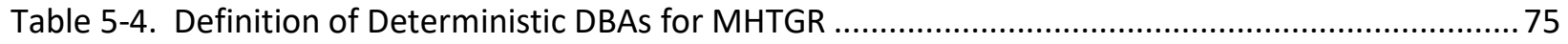

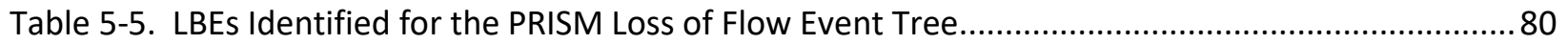

Table 5-6. Evaluation of SSCs Limiting Dose Release for PRISM DBE-1C.................................................. 81

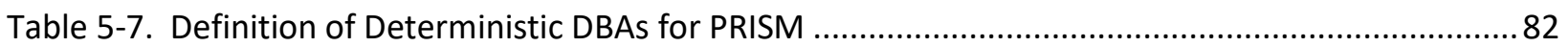




\section{List of Abbreviations}

\begin{tabular}{|c|c|c|c|}
\hline \multirow[t]{2}{*}{ ACRS } & Advisory Committee on Reactor & SSCs & Treatment SSCs \\
\hline & Safeguards & NST SSCS & Non-Safety-Related with No Special \\
\hline AOO & Anticipated Operational Occurrence & & Treatment SSCs \\
\hline ATWS & anticipated transient without scram & NTTF & Near Term Task Force \\
\hline BDBE* & Beyond Design Basis Event & PAG & Protective Action Guide \\
\hline BOP & balance-of-plant & PB & performance-based \\
\hline CDF & core damage frequency & PBMR & Pebble Bed Modular Reactor \\
\hline CFR & Code of Federal Regulations & PHA & process hazard analysis \\
\hline DBA & Design Basis Accident & POS & Plant Operating State \\
\hline DBE* & Design Basis Event & PRA & probabilistic risk assessment \\
\hline DBEHL & Design Basis External Hazard Level & PRISM & Power Reactor Innovative Small \\
\hline DID & Defense-in-Depth & & Module \\
\hline DOE & Department of Energy & PSF & PRA Safety Function \\
\hline$E A B$ & Exclusion Area Boundary & $\mathrm{QHO}$ & Quantitative Health Objectives \\
\hline ECA & Energy Conversion Area & RAI & Request for Additional Information \\
\hline EM & electromagnetic & RB & reactor building passive heat sink \\
\hline EPBE & Emergency Planning Basis Event & RCCS & Reactor Cavity Cooling System \\
\hline ES & Event Sequence & $\mathrm{RCS}$ & Reactivity Control System \\
\hline $\mathrm{F}-\mathrm{C}$ & Frequency-Consequence Target & RFDC & Required Functional Design Criteria \\
\hline Target & & RG & Regulatory Guide \\
\hline \multirow[t]{2}{*}{ FHR } & fluoride-salt-cooled, high-temperature & $\mathrm{RI}$ & risk-informed \\
\hline & reactor & RIPB & risk-informed and performance-based \\
\hline FMEA & failure modes and effects analysis & RIPB-DM & Risk-Informed and Performance-Based \\
\hline FSF & Fundamental Safety Function & & Integrated Decision-Making \\
\hline GDC & General Design Criteria & RPS & Reactor Protection System \\
\hline HAZOP & hazard and operability study & RSF & Required Safety Function \\
\hline HPB & helium pressure boundary & RSS & Reserve Shutdown System \\
\hline HTGR & high temperature gas-cooled reactor & RVACS & Reactor Vessel Auxiliary Cooling System \\
\hline HTS & Heat Transport System & SAP & Safety Assessment Principle \\
\hline IAEA & International Atomic Energy Agency & SBO & station blackout \\
\hline IE & Initiating Event & SCS & Shutdown Cooling System \\
\hline $\mathrm{LBE}^{*}$ & Licensing Basis Event & SG & steam generator \\
\hline LMP & Licensing Modernization Project & SGACS & Steam Generator Auxiliary Cooling \\
\hline LOHS & loss of heat sink & & System \\
\hline LOF & loss of primary forced flow & SR & Safety-Related \\
\hline LRF & large release frequency & SRDC & Safety-Related Design Criteria \\
\hline LWR & light water reactor & SRP & Standard Review Plan \\
\hline \multirow[t]{2}{*}{ MHTGR } & a specific prismatic mHTGR developed & SR SSCS & Safety-Related SSCs \\
\hline & by DOE & SSC & structures, systems, and components \\
\hline \multirow[t]{2}{*}{ mHTGR* } & modular high-temperature gas-cooled & TEDE & total effective dose equivalent \\
\hline & reactor & TI-RIPB* & technology-inclusive, risk-informed, \\
\hline MSRE & Molten Salt Reactor Experiment & & and performance-based \\
\hline MST & Mechanistic Source Term & TLST* & Top Level Safety Target \\
\hline NGNP & Next Generation Nuclear Plant & U.K. & United Kingdom \\
\hline NRC & Nuclear Regulatory Commission & U.S. & United States \\
\hline
\end{tabular}

*These terms have special meanings defined in this document. 


\subsection{EXECUTIVE SUMMARY}

\subsection{Introduction}

This report represents a key element in the development of a methodology for the efficient licensing of advanced non-light water reactors (non-LWRs). It is the result of a project led by Southern Company and cost-shared by the U.S. Department of Energy (DOE). This Licensing Modernization Project (LMP) will result in detailed proposals for establishing licensing technical requirements to facilitate efficient design and licensing of advanced non-LWRs. This report presents a modern, technology-inclusive, risk-informed, and performance-based (TI-RIPB) approach to identifying a full set of Licensing Basis Events (LBEs) used in the design and licensing of advanced non-LWRs. A key licensing outcome of this process is the structured, systematic, and reproducible process for the selection of LBEs, including Design Basis Accidents (DBAs), for advanced non-LWR plants. Additional LMP reports are planned to address other RIPB decisions within the licensing framework.

\subsection{Definition of Licensing Basis Events}

As the term is used in this document, LBEs are defined broadly to include all events used to support the safety aspects of the design" and to meet licensing requirements. They cover a comprehensive spectrum of events from normal operation to rare, off-normal events. There are four categories of LBEs:

- Anticipated Operational Occurrences (AOOs) - AOOs encompass anticipated event sequences expected to occur one or more times during the life of a nuclear power plant, which may include one or more reactor modules. Event sequences with mean frequencies of $1 \times 10^{-2} /$ plant-year and greater are classified as AOOs. AOOs take into account the expected response of all SSCs within the plant, regardless of safety classification.

- Design Basis Events (DBEs) - DBEs encompass infrequent event sequences that are not expected to occur in the life of a nuclear power plant, which may include one or more reactor modules, but are less likely than AOOs. Event sequences with mean frequencies of $1 \times 10^{-4} /$ plant-year to $1 \times 10^{-2} /$ plant-year are classified as DBEs. DBEs take into account the expected response of all SSCs within the plant regardless of safety classification.

- Beyond Design Basis Events (BDBEs) - BDBEs are rare event sequences that are not expected to occur in the life of a nuclear power plant, which may include one or more reactor modules, but are less likely than a DBE. Event sequences with mean frequencies of $5 \times 10^{-7} /$ plant-year to $1 \times 10^{-4} /$ plant-year are classified as BDBEs. BDBEs take into account the expected response of all SSCs within the plant regardless of safety classification.

- Design Basis Accidents - The DBAs for Chapter 15, "Accident Analyses," of the license application are postulated event sequences that are used to set design criteria and

\footnotetext{
${ }^{*}$ LBEs do not cover all events used to support the design, only those that meet safety requirements. There are other events considered in the design that do not necessarily impact safety performance but are used to ensure protection of the investment and to meet plant reliability, availability, and capacity factor targets.
} 
performance objectives for the design of Safety-Related (SR) SSCs. DBAs are derived from DBEs based on the capabilities and reliabilities of SR SSCs needed to mitigate and prevent event sequences, respectively. DBAs are derived from the DBEs by prescriptively assuming that only SR SSCs are available to mitigate postulated event sequence consequences to within the 10 CFR 50.34 dose limits.

The events evaluated within these categories are used to support various regulatory decisions associated with the design, operation, and siting of non-LWR plants.

\subsection{LMP Approach to Selecting and Evaluating LBEs}

The TI-RIPB approach to selecting and evaluating LBEs is designed to ensure that an appropriate set of events is identified for each reactor design and technology to support design and licensing decisions. The objective of this approach is the identification of the most risksignificant events ${ }^{*}$ for each design and technology to ensure that the appropriate limiting events can be selected for the DBAs. This is essential to ensure that risk insights are appropriately reflected in the design and licensing decisions. These decisions include not only the selection of the DBAs, but also the events to be considered in the formulation of design criteria for structures, systems, and components, and licensing requirements for protection against external hazards, siting evaluations, and emergency planning.

The AOOs, DBEs, and BDBEs are evaluated individually to support the tasks of assessing the performance of SSCs with respect to safety functions modeled in the PRA in response to Initiating Events (IEs) and event sequences and collectively demonstrate that the integrated risk of a multi-reactor module plant design meets the NRC Safety Goals and associated risk management targets. An important outcome of the selection and evaluation of LBEs is to identify design features of the plant that are necessary and sufficient to ensure that risk goals are achieved and licensing requirements are met. The AOOs and DBEs are used to establish the design bases for the plant, and the BDBEs are considered with the AOOs and DBEs to meet design risk targets and to support the evaluation of Defense-in-Depth (DID) adequacy. The use of these insights in the derivation of performance targets and design criteria for SSCs, including the radionuclide barriers, is a topic of a separate LMP report on SSC safety classification.

Performance requirements derived from the SSC classification process can be used in conjunction with Advanced Reactor Design Criteria (ARDC) to develop reactor design-specific Principal Design Criteria (PDC). The key licensing outcome is the systematic derivation of the DBAs and other events needed to formulate licensing requirements. The LBEs and associated design features included to address them also support performing environmental reviews and establishing necessary siting constraints, operational limits, and appropriate emergency planning requirements.

\footnotetext{
${ }^{*}$ In order to support a technology-inclusive approach, it is necessary to define risk significance in terms of technology inclusive risk metrics rather than LWR risk metrics such as core damage frequencies. The metrics used in the LMP methodology include the NRC Safety Goal Quantitative Health Objectives and the frequencies and consequences of LBEs. An approach to define risk significant LBEs is described in this report. Risk-significant SSCs are defined in the LMP report on SSC safety classification and performance criteria.
} 
On the basis of the lessons learned from a regulatory precedent review described in this report and the objectives of the LMP, the process for selecting LBEs for advanced non-LWRs should be:

- Systematic and reproducible

- Sufficiently complete

- Available for timely input to design decisions

- Risk-informed and performance-based

- Reactor technology-inclusive

- Consistent with applicable regulatory requirements

Figure 1-1 provides a flow chart indicating the tasks to identify and evaluate LBEs in concert with the design evolution. These tasks are intended to be carried out by the design and design evaluation teams responsible for establishing the key elements of the safety case and preparing a license application. The process is used to prepare an appropriate licensing document (e.g., licensing topical report) that documents the derivation of the LBEs, which would be reviewed by the regulator as part of the license review. The design and design evaluation teams are responsible for selecting the LBEs and justifying their selections. The regulator is responsible to review the design, the LBE selections, and their derivations. Although the NRC is expected to review the entire LBE selection and evaluation process, the specific tasks with increased regulatory involvement are indicated in the figure. 


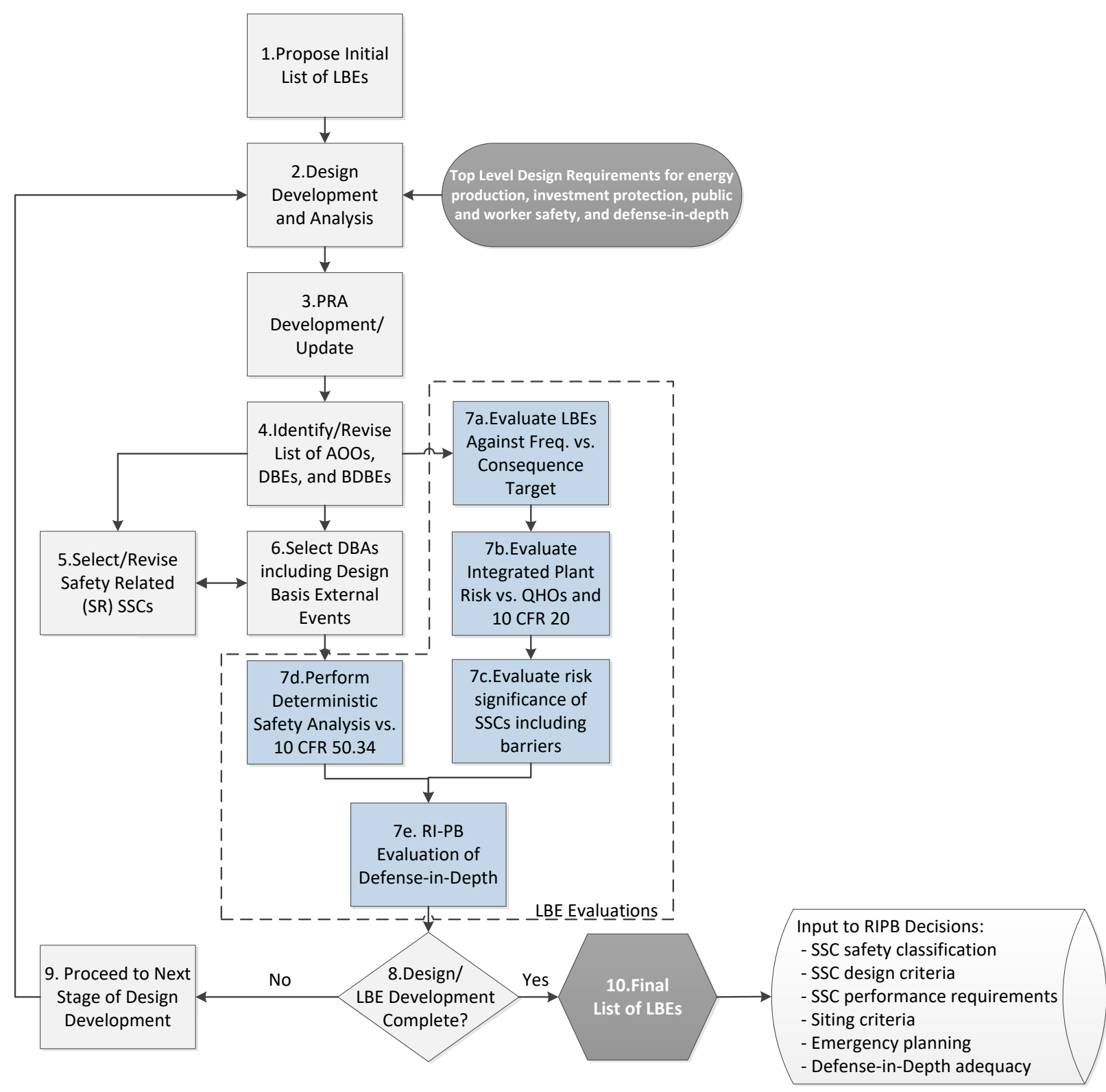

Figure 1-1. Process for Selecting and Evaluating Licensing Basis Events

The process is implemented in the following LBE selection and evaluation tasks:

\section{Task 1: Propose Initial List of LBEs}

During design development, it is necessary to select an initial set of LBEs which may not be complete but are necessary to develop the basic elements of the safety design. These events are to be selected deterministically and may be supported by qualitative risk insights based on all relevant and available experience, including prior experience from the design and licensing of reactors. The initial selection of events can also be supported by analysis techniques such as failure modes and effects analyses (FMEAs), hazard and operability studies (HAZOPs), and 
Master Logic Diagrams. In many cases, the designer may also have an initial assessment regarding which SSCs may be classified as Safety-Related to meet the safety design objectives for the reactor design. This classification would also be deterministically based and may be supported by qualitative risk insights using the same information utilized for the initial selection of LBEs.

\section{Task 2: Design Development and Analysis}

Design development is performed in phases and often includes a pre-conceptual, conceptual, preliminary, and final design phase and may include iterations within phases. Design development and analysis includes definition of the elements of the safety design approach, the design features to meet the top-level design requirements for energy production and investment protection, and analyses to develop sufficient understanding to perform a PRA and the deterministic safety analyses. The subsequent Tasks 3 through 10 may be repeated for each design phase or iteration until the list of LBEs becomes stable and is finalized. Because the selection of deterministic DBAs requires the selection of SR SSCs, this process also yields the selection of SR SSCs that are needed for the deterministic safety analysis in Task 7d.

\section{Task 3: Probabilistic Risk Assessment Development/Update}

The PRA may be introduced at any stage of design; however, the benefits of incorporation of risk insights into the design favor early introduction. A PRA model is developed and then updated as appropriate for each phase of the design. Prior to the first introduction of the PRA, it is necessary to develop a technically sound understanding of the potential failure modes of the reactor concept, how the plant would respond to such failure modes, and how protective strategies can be incorporated into formulating the safety design approach. The incorporation of safety analysis methods appropriate to early stages of design, such as FMEA and process hazard analysis (PHA), provide early stage evaluations that are systematic, reproducible, and as complete as the current stage of design permits. The PRA models the response of each SSC in the plant that performs a function to prevent or mitigate a release of radioactive material from any radionuclide source within the scope of the PRA. These SSC functions are defined in the LMP methodology as PRA Safety Functions (PSFs).

Developers are encouraged to begin developing the PRA early to support all design phases. However, developers have flexibility regarding when to introduce and develop the PRA to improve upon the initial risk management strategies or intentionally conservative analyses and related design features. If undertaken during the early design phases, the PRA is of limited scope, comprises a coarse level of detail, and makes use of engineering judgment much more than would a completed PRA that meets applicable PRA standards. The scope and level of detail of the PRA are enhanced as the design matures and siting information (or site envelope) is defined. For modular reactor designs, the event sequences modeled in the PRA should include event sequences involving single or multiple reactor modules or radionuclide sources. This approach provides useful risk insights into the design to ensure that event sequences involving multiple reactor modules are not risk-significant. The PRA process exposes sources of uncertainty encountered and provides estimates of the frequencies and doses for each LBE, including a quantification of the impacts of uncertainties using quantitative uncertainty analyses and supported by sensitivity analyses. 


\section{Task 4: Identify/Revise List of AOOs, DBEs, and BDBEs}

The event sequences modeled and evaluated in the PRA are grouped into event sequence families, each having a similar IE, challenge to the PSFs, plant response, end state, and mechanistic source term ${ }^{*}$ if there is a radiological release. Each of these families is assigned to an LBE category based on mean event sequence frequency of occurrence per plant-year summed over all the event sequences in the LBE family. The event sequence families from this task may confirm or revise the initial events identified in Task 1.

AOOs are off-normal events that are expected to occur in the life of the plant with frequencies exceeding $10^{-2} /$ plant-year, where a plant may be comprised of multiple reactor modules. DBEs are less frequent events that may occur in a plant with frequencies between $10^{-4}$ to $10^{-2} /$ plant-year. BDBEs are rare events with frequencies less than $10^{-4} /$ plant-year but with upper bound frequencies greater than $5 \times 10^{-7} /$ plant-year. LBEs could involve release of radioactive material and could involve two or more reactor modules or radionuclide sources. For LBEs with no radiological release, it is important to identify challenges to SSCs, including barriers that are responsible for preventing or mitigating a release of radioactive material. Such insights are important inputs to the subsequent task of identifying the Required Safety Functions (RSFs). The evaluation of the consequences of LBEs is supported by mechanistic source terms.

Event sequences with upper $95^{\text {th }}$ percentile frequencies less than $5 \times 10^{-7} /$ plant-year are retained in the PRA results and used to confirm that there are no cliff-edge effects. They are also taken into account in the RIPB evaluation of DID in Task 7e.

Tasks 5 and 6 are performed together rather than sequentially. Examples are provided in this report for high temperature gas-cooled reactors (HTGRs), sodium cooled fast reactors (SFRs), and molten salt reactors (MSRs) to illustrate how the tasks were implemented for these reactors.

\section{Task 5a: Identify Required Safety Functions}

In Task 5a, the full set of DBEs are examined to identify the PSFs that are necessary and sufficient to meet the F-C Target for all DBEs and high-consequence BDBEs, and to conservatively ensure that 10 CFR 50.34 dose requirements can be met. These PSFs are defined in the LMP methodology as Required Safety Functions (RSFs). High consequence BDBEs are those with consequences that exceed 10 CFR 50.34 dose criteria. For the DBEs, these PSFs, when fulfilled, are responsible for mitigating the consequences within the F-C Target. RSFs for any high-consequence BDBEs are responsible for preventing them from increasing in frequency into the DBE region and outside the F-C Target by exhibiting sufficient reliability performance to keep the BDBE frequency sufficiently low.

\section{Task 5b: Select/Revise Safety-Related SSCs}

For each of these RSFs identified in Task 5a, a decision is made on which set of SSCs is selected to perform these RSFs among those found to be available on each DBE. As a result of this selection, each DBEs is protected by a set of SR SSCs to perform each RSF. Structures and

\footnotetext{
* Designers and applicants may wish to consider "Accident Source Terms and Siting for Small Modular Reactors and Non-Light Water Reactors" (SECY-16-0012) for guidance on application of mechanistic source terms.
} 
physical barriers that are necessary to protect any SR SSCs in performing their RSFs in response to any design basis external event are also classified as SR. SR SSCs are also selected for any $\mathrm{RSF}$ associated with any high-consequence BDBEs in which the reliability of the SSC is necessary to keep the event in the BDBE frequency region. The remaining SSCs that are not classified as SR are considered in other evaluation tasks including Tasks 7b, 7c, 7d, and 7e. Those SSCs not classified as SR are further subdivided to identity additional safety significant SSCs that meet risk significance and DID adequacy criteria as explained more fully in the companion reports on SSC safety classification ${ }^{[6]}$ and DID evaluation. ${ }^{[7]}$ Performance targets and design criteria for both safety-related and non-safety-related SSCs are developed and described more fully in the SSC safety classification report.

\section{Task 6: Select DBAs and Design Basis External Hazard Levels}

For each DBE identified in Task 4, a deterministic DBA is defined that includes the RSF challenges represented in the DBE but assumes that the RSFs are performed exclusively by SR SSCs, and all non-safety-related SSCs that perform these same functions are assumed to be unavailable. These DBAs are then used in DBA analysis of the license application for supporting the conservative deterministic safety analysis.

NRC Regulatory Guide (RG) 1.203, “Transient and Accident Analysis Methods,” provides additional discussion of developing appropriate evaluation models for analyzing DBAs. The selection of conservative assumptions to be used in the DBA analysis will be informed by the quantitative uncertainty analysis of consequences that will be performed for the corresponding DBEs. In view of the fact that advanced non-LWRs will employ a diverse combination of inherent, passive, and active design features to perform the RSFs across layers of defense, and, taking into account the fact that the reactor safety design approach will be subjected to an evaluation of DID adequacy, the application of a single failure criterion is not deemed to be necessary.

A set of Design Basis External Hazard Levels (DBEHLs) will be selected to form an important part of the design and licensing basis. This will determine the design basis seismic events and other external events that the SR SSCs will be required to withstand. When supported by available methods, data, design, site information, and supporting guides and standards, these DBEHLs will be informed by a probabilistic external hazards analysis and will be included in the PRA after the design features that are incorporated to withstand these hazards are defined. Other external hazards not supported by a probabilistic hazard analysis will be covered by DBEHLs that are determined using traditional deterministic methods.

In many cases, it is expected that the initial selection of SR SSCs and selection of the DBAs will be based on a PRA that includes internal events but has not yet been expanded to address external hazards. With the understanding that SR SSCs are required to be capable of performing their RSFs in response to external events within the DBEHL, there will be no new DBAs introduced by external hazards. Some design basis external events such as external floods or seismic events may impact multiple reactor modules concurrently; therefore, a design objective would be to prevent a substantial release for such events. 
The codes and thermal hydraulic models used within the PRA will be subject to the technical adequacy requirements in the supporting PRA standards, whereas the codes and models used in DBA analysis are expected to satisfy RG 1.203 requirements for evaluation models.

\section{Task 7: Perform LBE Evaluations}

The deterministic and probabilistic safety evaluations that are performed for the full set of LBEs are covered in the following five sub-tasks:

\section{Task 7a: Evaluate LBEs against Frequency-Consequence Target}

In this task, the results of the PRA which have been organized into LBEs will be evaluated against an F-C Target as shown in Figure 1-2. The figure does not define specific acceptance criteria for the analysis of LBEs but rather serves as a tool to focus the attention of the designer and those reviewing the design and related operational programs to the most significant events and possible means to address those events. The NRC's Advanced Reactor Policy Statement includes expectations that advanced reactors should provide enhanced margins of safety. The safety margin between the design-specific PRA results and the F-C Target provides one useful and practical demonstration of how the design fulfills the NRC's expectations for enhanced safety.

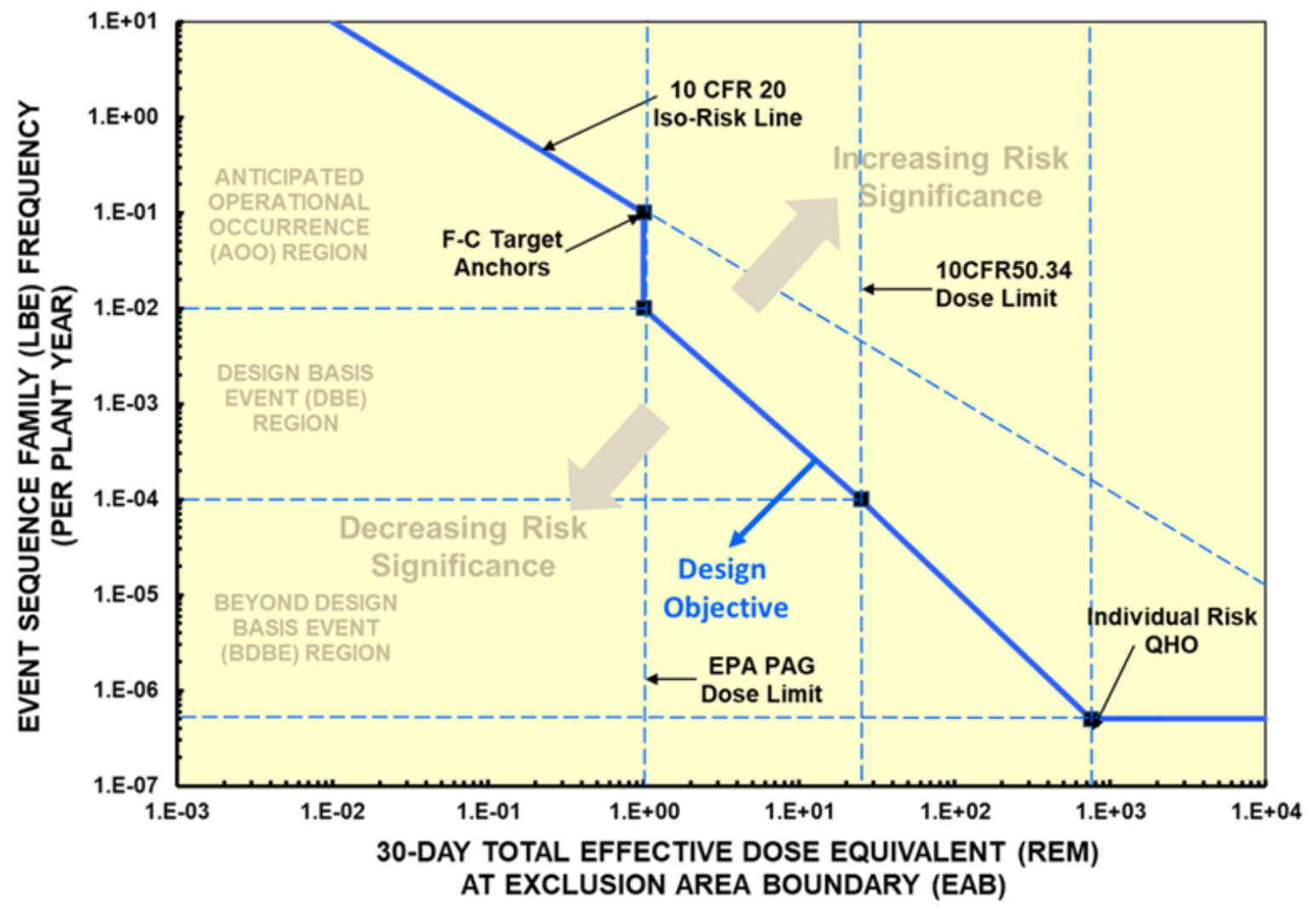

Figure 1-2. Frequency-Consequence Target Proposed for LMP 
These margins also are useful in the evaluation of DID adequacy in Task $7 \mathrm{~d}$. The evaluations in this task are performed for each LBE separately. The mean values of the frequencies are used to classify the LBEs into AOOs, DBEs, and BDBE categories. However, when the uncertainty bands defined by the $5^{\text {th }}$ percentile and $95^{\text {th }}$ percentile of the frequency estimates straddles a frequency boundary, the LBE is evaluated in both LBE categories. An LBE with mean frequency above $10^{-2} /$ plant-year and $5^{\text {th }}$ percentile less than $10^{-2} /$ plant-year is evaluated as an AOO and DBE. An LBE with a mean frequency less than $10^{-4} /$ plant-year with a $95^{\text {th }}$ percentile above $10^{-4} /$ plant-year is evaluated as a BDBE and a DBE. An event sequence family with a mean frequency less than $5 \times 10^{-7} /$ plant year but with a $95^{\text {th }}$ percentile frequency estimate above $5 \times 10^{-7} /$ plant-year is evaluated as a BDBE. Uncertainties about the mean values are used to help evaluate the results against the frequency-consequence criteria and to identify the margins against the criteria. The uncertainties about these means are considered as part of the RIPB DID evaluation in Task $7 \mathrm{e}$.

DBE doses are evaluated against the F-C Target based on the mean estimates of consequence. This approach is based on the fact that the use of a conservative dose evaluation is appropriate for the deterministic safety analysis in Task 7a but is not consistent with the way in which uncertainties are addressed in risk-informed decision-making in general, where mean estimates supported by a robust uncertainty analysis are generally used to support risk significance determinations. When evaluating risk significance, comparing risks against safety goal Quantitative Health Objectives (QHOs), and evaluating changes in risk against the RG 1.174 change in risk criteria, the accepted practice has been to first perform a quantitative uncertainty analysis and then to use the mean values to compare against the various goals and criteria, which are set in the context of uncertainties in the risk assessments. These assessments apply to both the frequency and consequence estimates.

The primary purpose of comparing the frequencies and consequences of LBEs against the F-C Target is to evaluate the risk significance of individual LBEs. The objective for this activity is that uncertainties in the risk assessments are evaluated and included in discussions of design features and operational programs related to the most significant events and possible compensatory measures to address those events.

The PRA process exposes sources of uncertainty encountered in the assessment of risk and provides estimates of the frequencies and doses for each LBE, including a quantification of the impacts of uncertainties using quantitative uncertainty analyses and supporting sensitivity analyses. Sources of uncertainty that are identified by the PRA and not fully resolved via quantification are addressed as part of a risk-informed evaluation of DID, as discussed more fully in the supporting report on DID. ${ }^{[7]}$ The evaluation of the consequences of all LBEs are supported by mechanistic source terms and a quantitative uncertainty analysis.

The upper bound consequences for each DBA, defined as the $95^{\text {th }}$ percentile of the uncertainty distribution, shall meet the 10 CFR 50.34 dose limit at the EAB. Sources of uncertainty in both frequencies and consequences of LBEs are identified and addressed in the LMP approach to DID. 
A function of the LBE frequency-dose evaluation is to ensure that LBEs involving radiological releases from two or more reactor modules do not make a significant contribution to risk and to ensure that measures to manage the risks of multi-reactor module or multi-source events are taken.

The final element of the LBE evaluation in this task is to identify design features that are responsible for keeping the LBEs within the F-C Target, including those design features that are responsible for preventing or mitigating risk-significant releases for those LBEs with this potential. This evaluation leads to performance requirements and design criteria that are developed within the process of the SSC classification task in the risk-informed, performancebased approach.

\section{Task 7b: Evaluate Integrated Plant Risk}

In this task, the integrated risk of all the LBEs, is evaluated against three cumulative risk targets:

- The total mean frequency of exceeding a site boundary dose of $100 \mathrm{mrem}$ from all LBEs should not exceed 1/plant-year. This metric is introduced to ensure that the consequences from the entire range of LBEs from higher frequency, lower consequences to lower frequency, higher consequences are considered. The value of $100 \mathrm{mrem}$ is selected from the annual cumulative exposure limits in 10 CFR 20.

- The average individual risk of early fatality within 1 mile of the EAB from all LBEs based on mean estimates of frequencies and consequences shall not exceed $5 \times 10^{-7} /$ plant-year to ensure that the NRC Safety Goal QHOs for early fatality risk is met.

- The average individual risk of latent cancer fatalities within 10 miles of the EAB from all LBEs based on mean estimates of frequencies and consequences shall not exceed $2 \times 10^{-6} /$ plant-year to ensure that the NRC Safety Goal QHOs for latent cancer fatality risk is met.

- One element of this task is to identify design features that are responsible for preventing and mitigating radiological releases and for meeting the integrated risk criteria. This evaluation leads to performance requirements and design criteria that are developed within the process of the SSC classification task in the LMP SSC safety classification report.

In addition to the two QHOs, the 10 CFR 20 criterion is considered in recognition that the referenced regulatory requirement is for the combined exposures from all releases even though it has been used in developing the F-C Target used for evaluating the risks from individual LBEs. Having these cumulative risk targets as part of the process provides a mechanism to ensure that the F-C Target is conservatively defined for use as a tool for focusing attention on matters important to managing the risks from non-LWRs.

\section{Task 7c: Evaluate Risk Significance of LBEs and SSCs Including Barriers}

In this task, the details of the definition and quantification of each of the LBEs in Task $7 \mathrm{a}$ and the integrated risk evaluations of Task $7 \mathrm{~b}$ are used to define both the absolute and relative risk significance of individual LBEs and SSCs and radionuclide barriers. These evaluations employ 
technology-inclusive risk-importance metrics and an examination of the effectiveness of each of the barriers in retaining radionuclides. LBEs are classified as risk-significant if the LBE site boundary dose exceeds 2.5 mrem over 30 days and the frequency of the dose is within $1 \%$ of the F-C Target. SSCs are classified as risk-significant if the SSC function is necessary to keep any LBEs inside the F-C Target, or if the total frequency of LBEs with the SSCs failed is within 1\% of any of the three cumulative risk targets identified in Task $7 \mathrm{~b}$. This information is used to provide risk insights to the design team and to support the RIPB evaluation of DID in Task 7e.

\section{Task 7d: Perform Deterministic Safety Analyses Against 10 CFR 50.34}

This task corresponds to the traditional deterministic safety analysis found in Chapter 15 of the license application. Deterministic analyses often use stylized scenarios for the purpose of demonstrating compliance with specific requirements, establish safety margins, and define equipment specifications and operational limits. Deterministic safety analysis can use conservative or best-estimate analytical methods with an appropriate accounting of uncertainties. The uncertainty analyses in the mechanistic source terms and radiological doses that are part of the PRA are available to inform the conservative assumptions used in this analysis and to avoid the arbitrary "stacking" of conservative assumptions that lack physical meaning.

\section{Task 7e: Risk-Informed, Performance-Based Evaluation of Defense-in-Depth}

In this task, the definition and evaluation of LBEs should be used to support an RIPB evaluation of DID. This task involves the identification of risk-significant sources of uncertainty in both the frequency and consequence estimates, and evaluation against DID criteria. Outcomes of this task include possible changes to the design to enhance the plant capabilities for DID, formulation of conservative assumptions for the deterministic safety analysis, and input to defining and enhancing programmatic elements of DID.

It is noted that this DID evaluation does not change the selection of LBEs directly. This evaluation could lead to compensatory actions that change the design capability or programmatic controls on the design, which in turn would lead to changes in the PRA and thereby affect the selection or evaluation of LBEs.

This may be a task where designers assess plant features for effective satisfaction of regulatory requirements such as 10 CFR 50.155, "Mitigation of Beyond-Design Basis Events," and 10 CFR 73, "Physical Protection of Plants and Materials." The results from the evaluation can also support related licensing matters such as defining appropriate constraints in terms of siting (i.e., 10 CFR 100, "Reactor Site Criteria"), offsite emergency planning, and development of plant procedures and guidelines.

The process and criteria to establish DID adequacy are discussed in the LMP report on DID.

\section{Task 8: Decide on Completion of Design/LBE Development}

The purpose of this task is to decide if additional design development is needed, either to proceed to the next logical stage of design or to incorporate feedback from the LBE evaluation that design, operational, or programmatic improvements should be considered. Such design improvements could be motivated by a desire to increase margins against the $\mathrm{F}-\mathrm{C}$ criteria, reduce 
uncertainties in the LBE frequencies or consequences, manage the risks of multi-reactor module events, limit the need for restrictions on siting or emergency planning, or enhance the performance against DID criteria. The DID adequacy evaluation may result in the need for additional iterations on the adequacy of design, operational, and programmatic programs, which in turn could influence the PRA and result in a need for cycling through some or all the LBE evaluation tasks.

\section{Task 9: Proceed to Next Stage of Design Development}

The decision to proceed to the next stage of design is reflected in this task. This implies not only completion of the design but also confirmation that DID criteria evaluated in Task $7 \mathrm{e}$ have been satisfied for that stage of design development. Open items or unverified assumptions should be resolved at later stages of design development.

\section{Task 10: Finalize List of LBEs and Safety-Related SSCS}

Establishing the final list of LBEs and SR SSCs signifies the completion of the LBE selection process and the selection of the SR SSCs. The next task in implementing the TI-RIPB approach is to formulate performance requirements and regulatory design criteria for SSCs that are necessary to limit LBE frequencies and doses. Important information from Task $7 \mathrm{~b}$ is used for this purpose.

\subsection{Motivation}

The NRC's 1995 PRA Policy Statement asserts that a probabilistic approach to regulation enhances and extends the traditional deterministic approach by allowing for consideration of a broader set of potential challenges to safety. This policy states:

"The Commission believes that an overall policy on the use of PRA methods in nuclear regulatory activities should be established so that the many potential applications of PRA can be implemented in a consistent and predictable manner that would promote regulatory stability and efficiency. In addition, the Commission believes that the use of PRA technology in NRC regulatory activities should be increased to the extent supported by the state of the art in PRA methods and data and in a manner that complements the NRC's deterministic approach."

The Policy Statement expresses further:

"A probabilistic approach to regulation enhances and extends this traditional, deterministic approach, by: (1) Allowing consideration of a broader set of potential challenges to safety, (2) Providing a logical means for prioritizing these challenges based on risk significance, and (3) Allowing consideration of a broader set of resources to defend against these challenges."

The LBE selection and evaluation approach presented in this report is guided by and is consistent with this policy. 


\subsection{INTRODUCTION}

\subsection{Purpose}

Many of the current regulatory requirements for US nuclear power plants are based on light water reactor (LWR) technology used for generation of electricity, necessitating changes to the LWR framework ${ }^{*}$ to facilitate efficient, effective, and predictable licensing expectations for a spectrum of novel, advanced, non-LWRs. The LMP, led by Southern Company and cost-shared by DOE and other industry participants, has proposed changes to specific elements of the current licensing framework and a process for implementation of the proposals. These proposals were originally described in a series of draft white papers that were reviewed by industry stakeholders and the NRC staff. Based on these reviews, industry guidance was prepared and documented in NEI $18-04^{[2]}$ gathering key aspects of the draft reports while addressing review comments in a form suitable for future NRC endorsement in a regulatory guide.

The LMP methodology documented in NEI 18-04 is intended to retain a high degree of nuclear safety, establish stable performance-based acceptance criteria, and enable near-term implementation of non-LWR design development, in support of national and industrial strategic objectives. The LMP objective is to support NRC in developing regulatory guidance for licensing advanced non-LWR plants.

The industry guidance in NEI 18-04 is technology-inclusive, risk-informed, and performancebased. The modernized framework is expressed in the form of a methodology and is technologyinclusive to accommodate the variety of technologies expected to be developed (implementation obviously will be technology-specific). It is risk-informed because it employs an appropriate blend of deterministic and probabilistic inputs to each decision. It is performance-based because it uses quantitative risk metrics to evaluate the risk significance of events and leads to formulation of performance targets on the capability and reliability of structures, systems, and components to prevent and mitigate accidents. By utilizing a risk-informed, performance-based approach for the Licensing Basis Event selection process the design and licensing efforts are more closely aligned with the safety objectives. The goal is efficient and effective development, licensing, and deployment of non-LWRs on aggressive timelines with even greater margins of safety than prior generations of technology. These goals fully support and reflect DOE and NRC visions for licensing and deploying advanced non-LWR plants.

The LMP methodology consists of elements including: establishment of TI-RIPB LBE selection; classification of structures, systems, and components; and establishment of predictable means to determine and preserve adequate DID. These process tasks are facilitated and informed by reports describing approaches and methods for: risk-informed decision making; the conduct

\footnotetext{
* "Framework" as used in the LMP products, refers to the interrelated elements that form the basis for the NRC's oversight of the use of radioactive materials, including the Atomic Energy Act and enabling legislation; licenses, orders, and regulations in Title 10 of the Code of Federal Regulations; regulatory guides, review plans, and other documents that clarify and guide the application of NRC requirements and amplify agency regulations; and licensing and inspection procedures and enforcement guidance. The focus of the LMP is primarily on new or amended regulatory guidance and implementation proposals (i.e., nearterm changes in actual regulation are not anticipated as part of LMP initiatives).
} 
and application of probabilistic risk assessments as part of the early and continuing lifecycle of new designs; and establishment of performance-based licensing criteria in lieu of LWR-centric prescriptive requirements. These elements are supported by reviews of past regulatory precedents and policies to make maximum use of existing approaches and NRC decisions, as well as assessments of current state of the art analytical tools. Gap analyses are used to identify where new or revised requirements are needed for a TI-RIPB methodology and propose changes in language or approach to allow the framework changes to be used effectively.

The relationship between the main topics described above is represented in Figure 2-1. A simple diagram cannot capture these relationships comprehensively because the development process for a licensing framework is iterative, not serial. As such, there are feedback loops that are difficult to represent in a simple figure, and some outputs are not shown. Nonetheless, this figure is intended to provide a generalized context for the major activities and how they fit into the overall methodology.

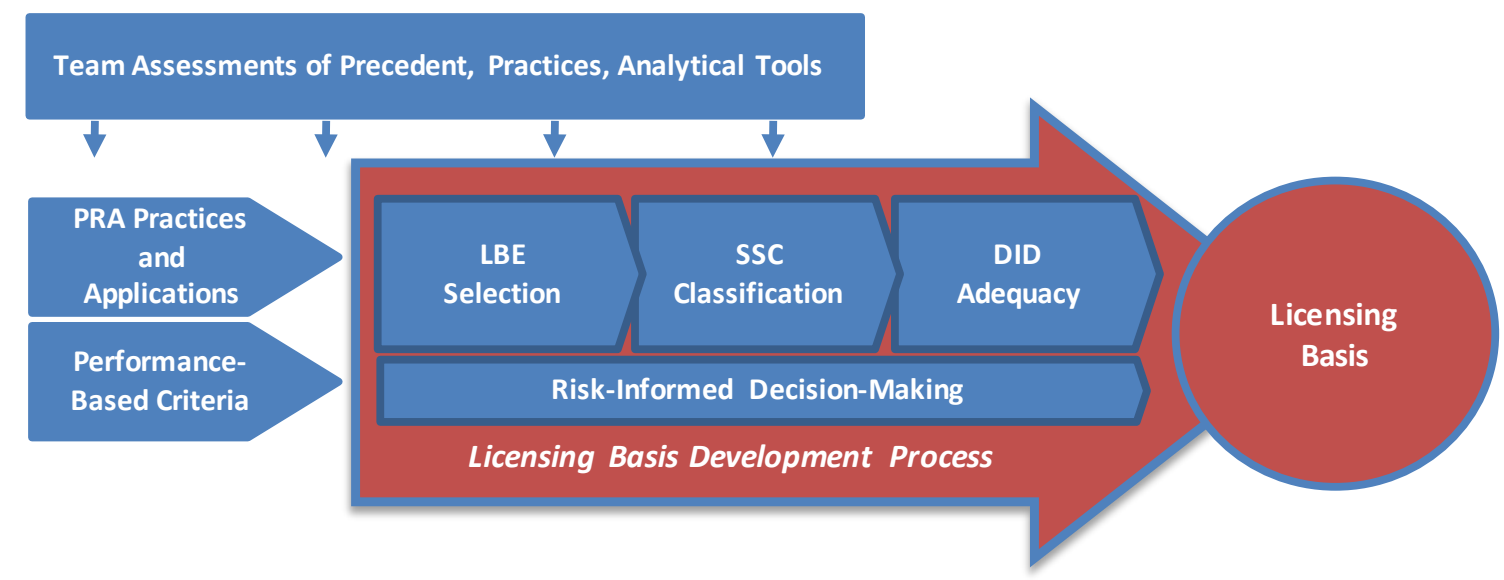

Figure 2-1. Elements of TI-RIPB Licensing Modernization Methodology

This report, "Modernization of Technical Requirements for Licensing of Non-Light Water Reactors: Licensing Basis Event Selection," represents a key element in development of a methodology for the efficient licensing of advanced non-LWRs. It is the result of a project led by Southern Company and cost-shared by the U.S. DOE. The LMP has provided guidance for establishing licensing technical requirements to facilitate efficient design and licensing of advanced non-LWRs in NEI 18-04. Such a guidance acknowledges enhancements in the level of safety achievable with advanced designs. It also reflects current knowledge regarding safety and design innovation, creating an opportunity for reduced regulatory complexity without diminishing levels of safety. The project builds on best practices as well as previous activities through DOE and industry-sponsored advanced reactor licensing initiatives.

This report reviews the relevant regulatory precedents for guidance in identifying LBEs to be considered, describes the methodology for selecting and classifying LBEs, and sets forth issues for discussion in order to facilitate an effective submittal leading to license applications for advanced non-LWRs. This report builds on the development and review of an LBE white paper for DOE's Next Generation Nuclear Plant (NGNP $)^{[3][3]}$ and is intended for use with a spectrum 
of advanced non-LWRs including modular HTGRs, MSRs, liquid-metal-cooled fast reactors, and other advanced non-LWRs.

A draft of this report in the form of a draft white paper was submitted for NRC review in 2017 and the NRC staff comments from this review are documented in Reference [1]. The guidance document for implementing the LMP methodology in NEI 18-04 includes a discussion on the LMP approach to selecting and evaluating LBEs that addresses the NRC comments on the draft LBE white paper. This LBE report reflects the clarifications of the LMP approach to LBE selection and evaluation identified in NEI 18-04. This approach makes use of the LMP approach to PRA development, which is the topic of a companion report. ${ }^{[5]}$ Although certain aspects of SSC safety classification are covered in this report, a more complete discussion of the LMP approach to SSC safety classification and performance criteria is found in Reference [6]. The LMP approach to evaluating DID adequacy is covered in Reference [7]. As discussed in these reports, the processes for PRA development, LBE selection and evaluation, SSC safety classification and performance targets, and evaluation of DID adequacy are highly integrated processes.

\subsection{Objective of this Paper}

The objective of this report is to provide a technology-inclusive, risk-informed, and performance-based (TI-RIPB) approach for the selection and evaluation of LBEs to support the preparation of license applications for advanced non-LWR plants. Included in this work is a systematic and reproducible process to achieve the following objective identified in the Standard Review Plan for Transient and Accident Analysis: ${ }^{[8]}$

"If the risk of an event is defined as the product of the event's frequency of occurrence and its consequences, then the design of the plant should be such that all the AOOs and postulated accidents produce about the same level of risk (i.e., the risk is approximately constant across the spectrum of AOOs and postulated accidents). This is reflected in the general design criteria (GDC), which generally prohibits relatively frequent events (AOOs) from resulting in serious consequences, but allows the relatively rare events (postulated accidents) to produce more severe consequences."

\subsection{Scope}

The approach described in this paper applies to a spectrum of advanced non-LWR designs, including mHTGRs, MSRs, liquid-metal-cooled fast reactors, and other known concepts, and is intended to be reactor technology-inclusive. This report discusses selection and classification of LBEs using criteria that focus on acceptable risks and consequences to the public. LBEs include all events considered in the design and licensing of the plant and include the prescriptive DBAs. The LBEs and associated design features included to address them also support establishing necessary operational limits, siting constraints, and appropriate emergency planning requirements. Considerations of the risks to the worker are outside the scope of the LMP methodology. 
Section 3 of this report provides an overview of the regulations and guidance considered during development of the proposed LBE selection and evaluation approach. This TI-RIPB approach is described in Section 4 and builds upon an approach that was developed for DOE's MHTGR and NGNP projects by incorporating lessons learned from NRC and Advisory Committee on Reactor Safeguards (ACRS) reviews of that approach and by considering its application in a reactor technology-inclusive manner. It also considers events and developments in the intervening period following the NGNP work, such as new insights from the Fukushima Accident and additional NRC regulatory methodology updates and studies. Section 4 includes a discussion of how both probabilistic and deterministic inputs are considered for informing the design and the events to be considered in licensing. Section 5 provides example LBEs that have been developed for HTGRs, SFRs, and MSRs using the LMP methodology. A summary of the LMP approach to selection and evaluation of LBEs is provided in Section 6.

\subsection{Summary of Outcome Objectives}

The LMP objective is to assist the NRC in developing regulatory guidance for licensing advanced non-LWR plants. This paper presents a modern TI-RIPB approach to identifying LBEs, which cover a spectrum of events considered in the design and licensing of a nuclear power plant. A key licensing outcome of this approach is the structured selection of DBAs that are traditionally analyzed in Chapter 15 of the license application.

The proposed LBE selection approach covers license applications for a single reactor and multireactor modular plants. *

This report has been prepared to demonstrate that:

- Each LBE is defined as an event sequence or event sequence family having a common or comparable and well-defined IE, plant response, challenge to reactor safety functions, and mechanistic source term, if the LBE involves a release.

- The structured, TI-RIPB process described in this document is a systematic approach for defining and evaluating the LBEs for advanced non-LWRs such as modular HTGRs, MSRs, and liquid-metal-cooled reactors.

- The LMP approach to defining LBEs is systematic and reproducible. As the term is used in this document, LBEs are defined broadly to include all the events used to support the safety aspects of the design and to meet licensing requirements. They cover a comprehensive spectrum of events from anticipated events to rare off-normal events. There are three categories of LBEs that can be addressed through the PRA or alternative risk assessment methodologies that involve conservative or best estimate approaches with appropriate accounting for uncertainties:

\footnotetext{
* "Plant," as the term is used in this document, means a nuclear plant that may or may not employ a modular design. "Modular design" means a nuclear power plant consists of two or more essentially identical nuclear reactors (modules) and each module is a separate nuclear reactor capable of being operated independent of the state of completion or operating condition of any other module co-located on the same site, even though the nuclear power plant may have some shared or common systems. ${ }^{[4]}$
} 
- Anticipated Operational Occurrences-AOOs encompass anticipated event sequences expected to occur one or more times during the life of a nuclear power plant, which may include one or more reactor modules. Event sequences with mean frequencies of $1 \times 10^{-2} /$ plant-year and greater are classified as AOOs. AOOs take into account the expected response of all SSCs within the plant, regardless of safety classification.

- Design Basis Events-DBEs encompass infrequent event sequences that are not expected to occur in the life of a nuclear power plant, which may include one or more reactor modules, but are less likely than AOOs. Event sequences with mean frequencies of $1 \times 10^{-4} /$ plant-year to $1 \times 10^{-2} /$ plant-year are classified as DBEs. DBEs take into account the expected response of all SSCs within the plant regardless of safety classification.

- Beyond Design Basis Events-BDBEs are rare event sequences that are not expected to occur in the life of a nuclear power plant, which may include one or more reactor modules, but are less likely than a DBE. Event sequences with mean frequencies of $5 \times 10^{-7}$ /plant-year to $1 \times 10^{-4}$ /plant-year are classified as BDBEs. BDBEs take into account the expected response of all SSCs within the plant regardless of safety classification.

The analyses of AOOs, DBEs, and BDBEs using a plant PRA or risk assessment methodology can also be used to assess potential accident consequences to support environmental reviews and decisions related to siting (e.g., 10 CFR 100.21, "Non-Seismic Site Criteria"), emergency planning, and protections against external hazards.

The fourth category of LBE that is defined and assessed using deterministic approaches is:

○ Design Basis Accidents - The DBAs for Chapter 15, "Accident Analyses," of the license application are postulated event sequences that are used to set design criteria and performance objectives for the design of SR SSCs. DBAs are derived from DBEs based on the capabilities and reliabilities of SR SSCs needed to mitigate and prevent event sequences, respectively. DBAs are derived from the DBEs by prescriptively assuming that only SR SSCs are available to mitigate postulated event sequence consequences to within the 10 CFR 50.34 dose limits. Alternatively, a designer may provide confidence DBAs do not result in offsite doses by showing that fission product barriers maintain integrity and thereby prevent the release of radioactive material. However, the specifics of how this robust barrier approach would be integrated into the LMP methodology for LBE selection and evaluation, SSC safety classification, and evaluation of DID adequacy have not been defined in the LMP methodology.

The DBAs are not selected on the basis of event frequency, but rather by a set of prescriptive rules similar to those employed in defining DBAs for existing LWRs. As shown with examples in this report for different types of advanced non-LWRs, they often correspond to event sequences modeled in the PRA with extremely low frequencies. An example of using best estimate approaches with an accounting for uncertainties in provided 
in RG 1.157, "Best Estimate Calculations of Emergency Core Cooling System Performance."

The LBEs in each category are evaluated individually to support the tasks of assessing the performance of SSCs with respect to PRA safety functions in response to IEs to meet applicable regulatory limits and collectively to demonstrate that the integrated risk of a multi-reactor module plant design meets the NRC Safety Goals. PRA safety functions are the safety functions modeled in the PRA that can prevent or mitigate the release of radioactive material from any radionuclide source within the scope of the PRA.

There will be different LBEs for events affecting single and multiple reactor modules or radiological sources. An important outcome of the selection and evaluation of LBEs is to identify design features of the plant that are necessary and sufficient to ensure that risk goals in the NRC Safety Goal Policy are achieved and licensing requirements are met. The LMP SSC report discusses the use of these insights in the derivation of performance targets and principal design criteria for SSCs, including the radionuclide barriers.

- Implementation of the proposed TI-RIPB approach to selecting LBEs requires the development of deterministic and probabilistic inputs to the LBE selections that have sufficient technical adequacy to support such decisions. The approach to performing the required PRA inputs and for achieving the necessary technical adequacy of the PRA is the topic of a companion LMP report on the approach to performing the PRA. The PRA or other risk assessment methodology is introduced at an early stage of the design to support design decisions and the level of detail and scope of the PRA is consistent with the level of detail of the design and site characterization.

- In order to address the selection of LBEs for a plant with two or more reactor modules or radionuclide sources, ${ }^{*}$ the frequencies of LBEs are expressed in units of events per plantyear where a plant is defined as a specific collection of reactor modules within the scope of the license application. ${ }^{\dagger}$ It is noted that non-core radionuclide hazards associated with some advanced reactor technologies may warrant greater emphasis in the selection of LBEs than is the case with current operating reactors. Thus, each LBE may involve a plant response or release from one or multiple reactors or radionuclide sources. The evaluation criteria on the frequency ranges for the LBE categories are as follows:

○ AOOs-Event sequences with mean frequencies greater than $10^{-2} /$ plant-year

○ DBEs-Event sequences with mean frequencies less than $10^{-2} /$ plant-year and greater than $10^{-4} /$ plant-year

○ BDBEs-Event sequences with mean frequencies less than $10^{-4} /$ plant-year and greater than $5 \times 10^{-7} /$ plant-year.

\footnotetext{
${ }^{*}$ Non-reactor sources include spent fuel storage, fuel processing, off-gas, and rad-waste processing and storage systems.

$\dagger$ Each reactor module may be separately licensed, but when the second and subsequent modules are licensed the multi-module LBEs will be defined, and the plant capabilities to ensure that multi-module accident risks are not significant will be incorporated into the design and licensing bases.
} 
- When the range of uncertainty in the estimated frequency of an LBE, as defined by the $95^{\text {th }}$ and $5^{\text {th }}$ percentiles of the uncertainty distribution, overlaps one of these LBE frequency boundaries, the LBE is evaluated in each affected LBE category.

- DBAs are deterministically defined and are not selected on the basis of frequency. However, the plant response to each DBA corresponds to either a DBE, BDBE, or lower frequency event sequence.

- Event sequence consequences for the LBE categories can be assessed by showing fission product barriers maintain integrity and thereby prevent the release of radioactive material. LBEs with radioactive material release can be assessed using an F-C Target to identify design and related operational programs needed to provide reasonable assurance of adequate protection of public health and safety or a cost-effective approach to address BDBEs. The F-C Target is a curve of LBE frequency vs. 30-day site boundary dose (TEDE) used to define the risk significance of LBEs and to incorporate risk management insights into the design. Key factors used to develop the F-C Target include:

- 10 CFR 20 which limits the total effective dose equivalent (TEDE) to individual members of the public from the licensed operation does not exceed $0.1 \mathrm{rem}(1 \mathrm{mSv})$ in a year. This regulation is used to establish F-C targets for AOOs. To avoid offsite impacts, doses from AOOs are limited by the EPA PAG dose limit of $1 \mathrm{rem}$. The use of 10 CFR 20 is made to evaluate the risks of LBEs individually and to evaluate the cumulative risks from all LBEs.

○ 10 CFR 50.34 which limits the TEDE to members of the public from a DBA to 25 rem. This regulation is used to establish F-C targets for DBEs.

- NRC Safety Goals and QHOs which limit the individual risks of early and latent cancer fatality due to radiation exposures to the population surrounding the nuclear power plant. These goals and objectives are used to establish F-C Targets for BDBEs.

- By identifying the margins between the LBE frequencies and consequences and the F-C target, this is one way to demonstrate the enhanced safety margins expected for advanced reactor technologies in the NRC's advanced reactor policy. ${ }^{[14]}$ In addition, as explained more fully in the companion LMP report on DID, evaluating these margins is an important aspect of demonstrating DID adequacy.

- It is emphasized that not all LBEs involve a release of radioactive material as evidenced by the examples presented in this report for two advanced reactor designs. An important aspect of evaluating each LBE is the development of a deep understanding of the safety features in the plant responsible for preventing a release as well as those features that limit the releases to acceptable levels. These insights are used to support SSC safety classification and to develop performance targets for SSCs responsible for preventing and mitigating releases as explained more fully in the companion LMP report on SSC safety classification.

- Designers may address design goals related to siting or emergency planning by showing that AOOs, DBEs, and BDBEs would not exceed specific dose acceptance criteria (e.g., $1 \mathrm{rem}$ at the site boundary) to limit the requirements for offsite emergency planning. 
- In addition to evaluating the risk significance of individual LBEs, the LMP approach to evaluating LBEs includes several cumulative risk targets to ensure that the integrated risk of the advanced non-LWR plant, which may be comprised of two or more reactor modules and radionuclide sources, is acceptably small and consistent with the NRC Advanced Reactor and Safety Goal policies. These cumulative risk targets include:

- The total frequency of exceeding of a site boundary dose of $100 \mathrm{mrem}$ from all LBEs shall not exceed 1/plant-year to ensure that the annual exposure limits in 10 CFR 20 are not exceeded.

$\circ$ The average individual risk of early fatality from all LBEs within the area 1 mile of the EAB shall not exceed $5 \times 10^{-7} /$ plant-year to ensure that the NRC Safety Goal QHO for early fatality risk is met.

- The average individual risk of latent cancer fatalities from all LBEs within the area 10 miles of the EAB shall not exceed $2 \times 10^{-6}$ /plant-year to ensure that the NRC Safety Goal QHO for latent cancer fatality risk is met.

- Designers may address design goals related to siting or emergency planning within the assumptions included in their risk assessments. The frequency below which events are not selected as BDBEs is $5 \times 10^{-7} /$ plant-year. The upper $95^{\text {th }}$ percentile estimate of the frequency is used for this purpose. Satisfaction of the NRC Safety Goal QHOs is assured when this frequency is not exceeded. The PRA examines events to $10^{-8} /$ plant-year to assure that there are no "cliff edge effects" just below this de minimis frequency.

- The kinds of events, failures, and natural phenomena that are evaluated include:

- Single, multiple, dependent, and common cause failures to the extent that these contribute to LBEs and their frequencies

- Events affecting one or more reactor modules or radionuclide source within the scope of the license application

- Internal events (including transients and accidents) and internal and external plant hazards that occur in all operating and shutdown modes and potentially challenge the capability to satisfactorily retain sources of radioactive material.

- External events require special consideration for establishing design specifications for structures, systems, and components and their treatment within safety classification and reliability programs

- Uncertainty distributions including upper and lower 95\% confidence values are evaluated for the frequency and the consequence for each $\mathrm{AOO}, \mathrm{DBE}$, and BDBE.

$\bigcirc$ The mean frequency is used to determine whether the event sequence family is an $\mathrm{AOO}, \mathrm{DBE}$, or BDBE. If the upper or lower bound on the LBE frequency straddles two or more regions, the LBE is compared against the frequency and consequence criteria for each region. 
O Sources of uncertainty that are identified by the PRA and not fully resolved via quantification are addressed as part of a risk-informed evaluation of DID as addressed in the LMP report on DID.

- The frequencies and consequences of event sequence families are assessed to identify design and related operational programs needed to provide reasonable assurance of adequate protection of public health and safety or a cost-effective approach to address BDBEs.

- The upper bound consequences for each DBA, defined as the $95^{\text {th }}$ percentile of the uncertainty distribution, shall meet the 10 CFR 50.34 dose limit at the EAB. Sources of uncertainty in both frequencies and consequences of LBEs are identified and addressed in the LMP approach to DID.

○ Key sources of uncertainty exposed by the application of the three risk triplet questions ("What can go wrong?", "What is the likelihood?", and "What are the consequences?") are addressed in the TI-RIPB approach to identifying and evaluating LBEs. Quantitative uncertainty analysis of LBE frequencies and consequences are performed and supplemented by sensitivity analyses to address sources of uncertainty that are not amenable to quantification. Key sources of uncertainty are addressed further in the evaluation of DID adequacy.

\subsection{Relationship to Other LMP Reports}

The LMP team prepared independent reports on each of the four major LMP elements. Additionally, the LMP team produced a narrative report describing the processes, events, and documents involved in producing the ultimate project deliverable product, NEI 18-04 "RiskInformed Performance-Based Technology Inclusive Guidance for Non-Light Water Reactor Licensing Basis Development." Finally, the LMP team produced a report based on the experiences of early adopters of the LMP RIPB process which includes best practices, lessons learned, and frequently asked questions and responses. See Table 1-1 for the Southern Company document numbers of each of these reports. 
Table 1-1. LMP Reports and Document Numbers

\begin{tabular}{|c|c|c|}
\hline Report Title & $\begin{array}{l}\text { Southern Company } \\
\text { Document Number }\end{array}$ & $\begin{array}{l}\text { DOE OSTI Document } \\
\text { Number }\end{array}$ \\
\hline Selection and Evaluation of Licensing Basis Events & SC-29980-100 Rev 1 & TBD \\
\hline Probabilistic Risk Assessment Approach & SC-29980-101 Rev 1 & TBD \\
\hline $\begin{array}{l}\text { Safety Classification and Performance Criteria for } \\
\text { Structures, Systems, and Components }\end{array}$ & SC-29980-102 Rev 1 & TBD \\
\hline $\begin{array}{l}\text { Risk-Informed and Performance-Based Evaluation of } \\
\text { Defense-in-Depth Adequacy }\end{array}$ & SC-29980-103 Rev 1 & TBD \\
\hline Final Project Report & SC-29980-105 Rev. 1 & TBD \\
\hline $\begin{array}{l}\text { LMP Lessons Learned, Best Practices, and Frequently } \\
\text { Asked Questions }\end{array}$ & SC-29980-106 Rev 0 & TBD \\
\hline
\end{tabular}

\section{LMP Approach to PRA Development ${ }^{[5]}$}

This report outlines the approach to develop a PRA for advanced non-LWR plants in support of risk-informed and performance-based (RIPB) applications. Future advanced non-LWR license applications will include a design-specific PRA that is capable of supporting the applications for NRC permit(s) or license(s). When introduced at an early stage of the design, the PRA is expected to result in a more efficient risk management process. This report provides insights on how to use a design specific PRA in support of the LMP application.

\section{LMP SSC Safety Classification and Performance Criteria Approach ${ }^{[6]}$}

Information developed from and used in the development of the PRA to define event sequences and evaluate their frequencies and consequences is an input to the SSC safety classification and development of SSC performance targets. Information from the PRA is used to establish the necessary and sufficient conditions of SSC capability and reliability in order for LBE frequencies, consequences, and uncertainties to stay within the frequency-consequence evaluation criteria derived from the TLRC and to implement risk management strategies to control the total integrated risk of the plant. Reliability targets for SSCs are determined based on the need to maintain each LBE within its LBE category (Anticipated Operational Occurrence, Design Basis Event, or Beyond Design Basis Event). RIPB SSC capability targets are defined in part by the selected design margins between the LBE frequencies and dose limits for that LBE category. Special treatment requirements for SSCs are derived to achieve the necessary and sufficient degree of reliability and capability of the SSCs. This is discussed in a companion report on the LMP SSC safety classification approach.

\section{LMP Evaluation of DID Adequacy[7]}

The PRA models and supporting assumptions are based in part on the plant capabilities for DID reflected in the design, as well as assumptions about the limits placed on design and operation of 
the plant by assumed programmatic DID measures. Information developed in the PRA is used to help evaluate the SSCs responsible for preventing and mitigating accidents. The PRA also plays an important role in the identification of key sources of uncertainty, and this supports a feedback loop to identify possible enhancements to plant capability and programmatic aspects of DID. Hence, the PRA provides important input to the risk-informed evaluation of DID, complements the NRC's deterministic approach and traditional DID philosophy, and provides a more objective, RIPB means to systematically demonstrate DID adequacy and preservation. This is discussed in a companion report on the LMP approach to evaluating DID adequacy.

\section{LMP Final Report}

The LMP team produced a narrative report describing the processes, events, and documents involved in producing the ultimate Project deliverable product, NEI 18-04 "Risk-Informed Performance-Based Technology Inclusive Guidance for Non-Light Water Reactor Licensing Basis Development." This report contains a wealth of references to documents that future users of the LMP RIPB process may find useful. Tables within the report provide references to the NRC Agencywide Document Management System (ADAMS) Accession Numbers of many industry and NRC documents that future permit and license applicants may wish to reference in their own applications.

\section{LMP Lessons Learned, Best Practices, and Frequently Asked Questions with Responses}

The LMP team produced a report based on the experiences of early adopters of the LMP RIPB process which includes best practices, lessons learned, and frequently asked questions with responses. This report provides guidance to reactor designers on how to efficiently implement the LMP RIPB processes within their own organization and answers to thirty-two frequently asked questions from reactor designers. 


\subsection{REGULATORY FOUNDATION AND PRECEDENTS}

There is a substantial set of prior activities, policies, practices, and precedents stretching more than 30 years back in time that inform RIPB processes and uses. NRC and international regulations, policies, guidance, and other precedents that are relevant to the definition of LBEs and their treatment are discussed in this section. NRC and ACRS feedback on previous efforts to define LBEs for advanced non-LWRs are also reviewed for LBE definition guidance. This regulatory background is examined to investigate two aspects of the proposed TI-RIPB approach for the LMP. The first is the process of defining and selecting the LBEs and the second is the development of the Top-Level Safety Targets ${ }^{*}$ (TLSTs) that are used to establish evaluation boundaries on the frequencies and radiological consequences for classifying and evaluating the LBEs.

The scope of this review includes U.S. regulatory requirements and supporting policies, NRC directives, regulatory guidance, and the Standard Review Plan, as well as international safety standards. Insights from NRC pre-licensing reviews of advanced non-LWRs are also included. This section of the report builds on the regulatory review in the NGNP white paper on LBE selection ${ }^{[3]}$ by incorporating more recent developments and precedents and by considering the need to have a reactor technology inclusive approach for selecting LBEs rather than one focused on HTGR-specific technology only. Observations and conclusions reached from this review that are used in the definition of the LBE approach are summarized at the end of this section.

\subsection{Regulatory Foundation and Precedent Review Summary}

This section reviews NRC requirements and other relevant precedents for insights on how to select LBEs for a new reactor design. This review reflects on the qualitative approach to risk used in the past, relying on judgment and prescription derived from years of LWR design, analysis, and operations. The purpose is not to criticize, but rather to identify desirable attributes of a TI-RIPB approach to the selection of LBEs.

NRC regulatory requirements for the design of currently licensed and new reactors refer to several different kinds of events included within the licensing basis including AOOs, DBEs, postulated accidents, DBA, and BDBE. There are significant differences in licensing event terminology between NRC documents and LMP as shown in Table 3-1.

\footnotetext{
* The LMP term "Top Level Safety Targets" was referred to in the Next Generation Nuclear Plant (NGNP) project as "Top Level Regulatory Criteria (TLRC)." The LMP changed the term to TLST emphasize that the frequency-consequence criteria derived from these inputs are to be used as design targets and are not considered regulatory limits.
} 
Modernization of Technical Requirements

\section{Table 3-1. Definitions of Licensing Basis Events}

\section{Event Type NRC Definition}

"Conditions of normal operation that are expected to occur one or more

Anticipated

Operational

Occurrences

(AOOs)

times during the life of the nuclear power unit ${ }^{*}$ and include but are not

limited to loss of power to all recirculation pumps, tripping of the turbine

generator set, isolation of the main condenser, and loss of all offsite power." [SRP 15.0 and 10 CFR 50 Appendix A]

"Conditions of normal operation, including AOOs, design-basis accidents, external events, and natural phenomena, for which the plant must be

Design Basis designed to ensure functions of safety-related electric equipment that

Events ensures the integrity of the reactor coolant pressure boundary; the

(DBEs) capability to shut down the reactor and maintain it in a safe shutdown condition; or the capability to prevent or mitigate the consequences of accidents that could result in potential offsite exposures." [SRP 15.0]

"This term is used as a technical way to discuss accident sequences that are possible but were not fully considered in the design process because

Beyond

Design Basis

Events

(BDBEs) they were judged to be too unlikely. (In that sense, they are considered beyond the scope of design-basis accidents that a nuclear facility must be designed and built to withstand.) As the regulatory process strives to be as thorough as possible, 'beyond design-basis' accident sequences are analyzed to fully understand the capability of a design." [NRC Glossary]

"Postulated accidents that are used to set design criteria and limits for the design and sizing of safety-related systems and components."

Design Basis [SRP 15.0]

Accidents "A postulated accident that a nuclear facility must be designed and built

(DBA) to withstand without loss to the systems, structures, and components necessary to ensure public health and safety." [NRC Glossary and NUREG-2122]

Licensing

Basis Events Term not used formally in NRC documents.

(LBEs)
LMP Definition

Anticipated event sequences expected to occur one or more times during the life of a nuclear power plant, which may include one or more reactor modules. Event sequences with mean frequencies of $1 \times 10^{-2} /$ plant-year and greater are classified as AOOs. AOOs take into account the expected response of all SSCS within the plant, regardless of safety classification.

Infrequent event sequences that are not expected to occur in the life of a nuclear power plant, which may include one or more reactor modules, but are less likely than AOOs. Event sequences with mean frequencies of $1 \times 10^{-4} /$ plant-year to $1 \times 10^{-2} /$ plant-year are classified as DBEs. DBEs take into account the expected response of all SSCs within the plant regardless of safety classification.

Rare event sequences that are not expected to occur in the life of a nuclear power plant, which may include one or more reactor modules, but are less likely than a DBE. Event sequences with mean frequencies of $5 \times 10^{-7} /$ plantyear to $1 \times 10^{-4} /$ plant-year are classified as BDBEs. BDBEs take into account the expected response of all SSCs within the plant regardless of safety classification.

Postulated event sequences that are used to set design criteria and performance objectives for the design of SR SSCS. DBAs are derived from DBEs based on the capabilities and reliabilities of SR SSCs needed to mitigate and prevent event sequences, respectively. DBAs are derived from the DBEs by prescriptively assuming that only SR SSCS are available to mitigate postulated event sequence consequences to within the 10 CFR 50.34 dose limits.

The entire collection of events considered in the design and licensing basis of the plant, which may include one or more reactor modules. LBEs include AOOs, DBEs, BDBEs, and DBAs.

*SRP 15.0 further breaks down AOOs into events with "moderate" frequency (events expected to occur several times during the plant life) and "infrequent" (events that may occur during the plant life). 
For normal operations, including AOOs, the NRC regulations are, for the most part, generic and appear to generally apply to an advanced non-LWR plant. The applicant is required to classify the events considered within the design basis as either AOO or accident (DBA) based on a qualitative and presumably subjective assessment of the expected frequency of occurrence because there are no quantitative frequency criteria included. In many cases it is unclear whether the qualitative characterization of frequency refers to that for an IE or for an entire accident sequence. While the applicant's classification is subjected to NRC staff review there is no quantification of the event frequencies nor a prescribed method for ensuring that design specific events are adequately considered. A concern for advanced non-LWRs is that events that are uniquely appropriate for a given reactor technology are likely not represented on the supplied lists of generic LWR events, so it is necessary to have a method that is systematic and reproducible to derive the appropriate list of LBEs. For non-LWR plants whose designs depart in major ways from those of existing and even advanced non-LWRs, a more systematic and quantitative means of identifying the unique events and correctly classifying their frequencies would be necessary to ensure a safe design and contribute to a more predicable path to a license.

Moreover, establishing an appropriate set of reactor technology specific LBEs cannot wait until the submittal of a license application. This selection is essential to the development of any design and must be established early in the design process. ${ }^{*}$

All the example events given in the definition of $\mathrm{AOO}$ in the regulations, supporting regulatory guides, and Standard Review Plan ${ }^{[8]}$ are applicable to LWRs. While some of these may apply, many may not be applicable to a particular non-LWR design and such events are unlikely to be limiting for a non-LWR design.

In the selection of LBEs it is expected that the selection will consider a comprehensive and exhaustive set of events from which to identify the limiting events. However, specific criteria for how to determine which events are limiting are not provided in existing regulatory guidance. In addition, it is not clear from the regulatory guidance which events are considered to be limited by the selected events. This points to a need for a systematic and reproducible process to identify the DBAs for the deterministic safety analysis.

With few exceptions, such as provisions for protection against natural phenomena and inclusion of some generic events in the lists of example events such as loss of offsite power and station blackout, the regulations that have evolved for unplanned transients and accidents are light water reactor-specific. The GDC define the types of design considerations that apply to the design of SSCs that prevent or mitigate a specified set of postulated accidents. For example, GDC typically indicate that safety systems must be able to perform their design basis functions given a single active failure and a concurrent loss of offsite power.

\footnotetext{
* One additional definition is required to understand the importance of the terms. The "design bases" means that information which identifies the specific functions to be performed by a structure, system, or component of a facility, and the specific values or ranges of values chosen for controlling parameters as reference bounds for design. These values may be (1) restraints derived from generally accepted state-of-the-art practices for achieving functional goals, or (2) requirements derived from analysis (based on calculation and/or experiments) of the effects of a postulated accident for which a structure, system, or component must meet its functional goals. [10 CFR 50.2 Definitions]
} 
NRC's regulations do not have performance-based criteria to limit the consequences of BDBEs nor quantitative criteria for classifying events as BDBEs based on frequency other than noting they were considered too infrequent to be included in the design basis. In apparent response to events that have occurred but had not been anticipated in the original design and licensing bases, regulations have been added to provide protection against selected BDBEs. Examples of these include anticipated transients without scram (ATWS) addressed in 10 CFR 50.62 ${ }^{[10]}$ and station blackout (SBO) addressed in 10 CFR 50.63. ${ }^{[11]}$

The regulations associated with licensing events and their supporting regulatory documents do not distinguish well between events and event sequences for the purpose of characterizing the frequency of occurrence and classifying as either an AOO, DBA, or BDBE. The term "event sequence" is referred to here in the context of analyzing how the plant responds to IEs. The point here is a given event may be characterized at a certain frequency level and severity of plant impact, but when compounded by additional failures both the frequency and the level of impact are different. Hence, there may be different LBEs having different levels of frequency and severity stemming from the same IE. In reviewing the regulatory documents, it is extremely difficult to sort out in most cases whether the term "events" refer to IEs only or to some sequence of events. A goal of the LMP is to consider IEs and the associated event sequences as distinct challenges to the safety functions in order to provide sufficient completeness in the identification of LBEs.

In many cases, the events classified as AOOs or DBAs as discussed in the regulations and supporting SRP are referred to as "initiating events." By applying the single failure criterion, the safety analysis for the DBAs includes the requirement that the "worst" active single failure be assumed in demonstrating that safety criteria are met. However, the probability of the single failure does not have any bearing on the classification of the event. In addition, non-safetyrelated SSCs including offsite power supplies are assumed not to be available in the deterministic safety analysis of DBAs, which also would be considered if the frequency of the DBA were to be assessed.

With few exceptions, there does not appear to be a consideration of the probability of a common cause failure that could occur in combination with an IE to produce a DBA even though the service experience indicates that there have been many occurrences of such events. The application of the single failure criterion for DBAs seems to assume that common cause failures will be prevented by meeting the design requirements. In the limited cases of selected BDBE requirements, such as those for ATWS and SBO, event sequences that could be caused in part by a common cause failure and involve multiple failures of redundant components are identified as being comprised of a sequence initiated by an AOO (transient for ATWS and loss of offsite power for SBO). However, a systematic way to consider both events and event sequences that could be comprised of combinations of single failures and common cause failures is not included in the enumeration of prescriptive events nor in the characterization of their frequencies or level of severity of the challenge.

An important insight from this review is that, based on what can be gleaned from the regulations and supporting documents, the historical approach to selecting an appropriate set of LBEs for a given design is ad hoc. The challenge facing designers and licensees for advanced non-LWRs is 
to find a process for selecting LBEs that is systematic, reproducible, and capable of identifying the appropriate limiting events for a given design.

\subsection{Summary of Documents Reviewed}

A summary of the documents reviewed for regulatory guidance and insights from relevant precedents is provided in Table 3-2. The regulatory documents include the U.S. CFR, NRC policies and policy statements, NRC Staff Requirements Memoranda, regulatory guides, the SRP (NUREG-0800), and relevant ACRS letters. The relevant regulatory precedents include initiatives to develop RIPB licensing approaches for MHTGR, Power Reactor Innovative Small Module (PRISM), PBMR, DOE NGNP, and the NRC and ACRS reviews and feedback on those initiatives.

\section{Table 3-2. Documents Reviewed for Regulatory Bases and Precedents}

\begin{tabular}{|c|c|c|}
\hline Category & Reference & Applicable Content ${ }^{*}$ \\
\hline \multirow{15}{*}{$\begin{array}{l}\text { NRC } \\
\text { Regulations }\end{array}$} & 10 CFR 50 Appendix A & AOO definition \\
\hline & 10 CFR 50.34 & Dose limits for postulated accidents \\
\hline & 10 CFR 50.44 & Requirements for combustible gas control \\
\hline & 10 CFR 50.62 & Requirements for ATWS \\
\hline & 10 CFR 50.63 & Requirements for SBO \\
\hline & 10 CFR 50.150 & Requirements for aircraft impact assessment \\
\hline & 10 CFR 52.1 & Definitions for reactor unit, modular design \\
\hline & 10 CFR 63 & $\begin{array}{l}\text { Frequency and dose performance requirements for Yucca Mountain } \\
\text { Pre-closure Safety Analysis }\end{array}$ \\
\hline & 10 CFR 63.111 & Performance objectives for geologic repository \\
\hline & 10 CFR 63.112 & Performance objectives for pre-closure operations \\
\hline & 10 CFR 20 & Annual dose limits for normal operation and AOOs \\
\hline & 10 CFR 50 Appendix I & Design objectives for keeping releases as low as reasonably achievable \\
\hline & 10 CFR 52.79 & Principal design criteria for SSCs to limit doses \\
\hline & 10 CFR 100 & Dose limits for defining EAB and low population zone \\
\hline & 40 CFR 190 & Environmental radiation protection standards \\
\hline \multirow{4}{*}{ NRC Policies } & 73 FR 60612 & Policy on regulation of advanced reactors \\
\hline & 60 FR 42622 & Policy on use of PRA \\
\hline & $51 \mathrm{FR} 28044$ & Safety goal policy \\
\hline & 50 FR 32138 & Severe accident policy \\
\hline \multirow{10}{*}{$\begin{array}{l}\text { NRC Policy } \\
\text { Statements }\end{array}$} & SRM/SECY 90-16 & Evolutionary LWR certification issues \\
\hline & SECY 2002-0076 & Semi-annual update on future licensing \\
\hline & SECY 2003-0047 & Policy issues related to non-LWR licensing \\
\hline & SRM 2003-0047 & Staff requirements memorandum for SECY 2003-0047 \\
\hline & SECY 2005-0006 & Regulatory structure and policy issues for new plant licensing \\
\hline & SECY 2010-0034 & Policy, licensing, and technical issues for SMRs \\
\hline & SECY 2011-0079 & License structure for multiple module SMRs \\
\hline & SECY 2011-0152 & Emergency planning for SMRs \\
\hline & SECY 2013-0029 & History of large release frequency metric \\
\hline & SECY 2016-0012 & Accident source terms for SMRs and non-LWRs \\
\hline
\end{tabular}




\begin{tabular}{|c|c|c|}
\hline Category & Reference & Applicable Content ${ }^{*}$ \\
\hline \multirow{8}{*}{$\begin{array}{l}\text { NRC } \\
\text { Guidance }\end{array}$} & NUREG-0800, Chapter 15 & Transient and Accident Analysis \\
\hline & NUREG-0800, Chapter 19 & PRA and severe accident evaluation \\
\hline & Reg. Guide 1.174 & Use of PRA in risk-informed decisions approach \\
\hline & Reg. Guide 1.200 & Technical adequacy of PRA \\
\hline & NUREG/BR-0303 & Performance-based regulation guidance \\
\hline & NUREG-1860 & RIPB regulatory structure feasibility study \\
\hline & NUREG-2150 & Proposed risk management regulatory framework \\
\hline & NRC NTTF Report & Review of Fukushima Daiichi accident \\
\hline ACRS & ACRS letter April 22, 2004 & $\begin{array}{l}\text { ACRS views on risk metrics for non-LWRs and interpretation of Safety } \\
\text { Goal QHOs }\end{array}$ \\
\hline \multirow{5}{*}{ NGNP } & INL/EXT-09-17139 & Defense-in-Depth white paper \\
\hline & INL/EXT-10-19521 & LBE white paper \\
\hline & INL/EXT-11-21270 & PRA white paper \\
\hline & INL/EXT-13-28205 & NRC licensing status summary \\
\hline & ACRS Letter May 15, 2013 & ACRS views on NGNP proposed licensing approach \\
\hline \multirow{5}{*}{ PBMR } & Exelon Letter Jan. 31, 2002 & PBMR RIPB licensing approach \\
\hline & NRC Letter Sept. 24, 2007 & RAls regarding PBMR white papers \\
\hline & PBMR Limited Letter & Response to RAls from Sept. 24, 2007 \\
\hline & March 21, 2008 & \\
\hline & NRC Letter March 26, 2002 & NRC preliminary findings on licensing approach \\
\hline \multirow{4}{*}{ MHTGR } & DOE-HTGR-86-024 & Preliminary safety information for MHTGR \\
\hline & DOE-HTGR-86-011 & PRA for MHTGR \\
\hline & DOE-HTGR-86-034 & Licensing Basis Events for MHTGR \\
\hline & NUREG-1338 & Draft Pre-application safety evaluation for MHTGR \\
\hline \multirow{2}{*}{ PRISM } & NUREG-1368 & Pre-application safety evaluation for PRISM liquid metal reactor \\
\hline & GE-Hitachi 2017 report & Development and modernization of PRISM PRA \\
\hline \multirow{3}{*}{$\begin{array}{l}\text { Yucca } \\
\text { Mountain }\end{array}$} & DOE/RW-0573 & Yucca Mountain Repository Safety Analysis Report \\
\hline & NUREG-2108 & NRC technical evaluation of Yucca Mountain Safety Analysis Report \\
\hline & NUREG-1804 & Yucca Mountain review plan \\
\hline \multirow{3}{*}{$\begin{array}{l}\text { Industry } \\
\text { Consensus } \\
\text { Standards }\end{array}$} & ASME/ANS RA-Sb-2013 & PRA standard for operating LWR plants \\
\hline & ASME/ANS RA S-1.4-2013 & Trial use PRA standard for advanced non-LWR plants \\
\hline & ANSI/ANS-53.1-2011 & Nuclear safety design process for modular helium cooled reactors \\
\hline \multirow{3}{*}{$\begin{array}{l}\text { International } \\
\text { Guidance }\end{array}$} & IAEA NSR-1 & Nuclear safety design requirements \\
\hline & United Kingdom SAPs & United Kingdom Safety Assessment Principles \\
\hline & Farmer 1967 Paper & Proposal for a frequency-consequence risk criterion \\
\hline \multirow{3}{*}{\multicolumn{2}{|c|}{$\begin{array}{l}\text { * Abbreviations used in table: } \\
\text { IAEA-International Atomic Energy Agency } \\
\text { HTGR-high temperature gas-cooled reactor }\end{array}$}} & NTTF-Near Term Task Force \\
\hline & & RAI-Request for Additional Information \\
\hline & & SAP-Safety Assessment Principle \\
\hline
\end{tabular}

Additional insights were developed by reviewing the use of PRA to perform the Pre-Closure Safety Analysis, which was required for licensing the Yucca Mountain Repository as well as the frequency-consequence criteria that were incorporated into the regulations for that facility. International perspectives were incorporated into the review based on relevant documents from the International Atomic Energy Agency (IAEA) and the regulatory authority in the United Kingdom (U.K.). A full discussion of the LBE selection and evaluation insights derived from these documents is found in Appendix A. The conclusions from this review are presented in the next section. 


\subsection{Precedent Review Summary}

The following observations and conclusions are made in this review of the regulatory foundation for selection of LBEs for advanced non-LWRs. These observations and conclusions shape the development of an approach for LBE selection that is provided in Section 4.0.

- Existing NRC policy and strategy statements fully support the greater use of RIPB practices. This vision is clearly articulated in NUREG-2150. There has been partial development of RIPB methods for the backfit, operation, oversight, and modification of existing LWRs; however, little or no guidance for RIPB decision-making has been established for new, non-LWR advanced designs.

- Current U.S. regulations and regulatory guidance ("framework") for LWR-based designs do not include or provide a reproducible approach for selecting LBEs for advanced nonLWRs or for ensuring that advanced non-LWRs of differing designs would be treated in a consistent manner for establishing their design and licensing bases.

- One reactor technology-inclusive set of regulatory documents that was identified in this regulatory review is that reflected in the U.K. SAPs. The SAPs include numerical targets for evaluating LBE frequencies and consequences, which differentiate between those to be applied to each reactor unit and those that apply to the site as a whole. Different targets are expressed for regulatory evaluation boundaries and design objectives, thereby capturing the notion that risks are not to be used as a strict pass-fail acceptance test.

- The approach that was developed for the MHTGR and advanced for the Exelon, PBMR, and NGNP projects, as well as the approach used for PRISM LBE selection, provide an appropriate baseline from which to develop the LBE selection process for advanced reactor design and licensing. An LBE selection approach proposed in NUREG-1860 was also reviewed for insights to help define desirable attributes of an effective LBE selection process. This regulatory foundation review provides guidance for refining and advancing these approaches.

- The RIPB approach advanced in the MHTGR, PBMR, and NGNP projects has been reflected in a design standard for MHRs in ANSI/ANS-53.1. This standard provides specific design criteria for implementing the approach that is consistent with the approach described in the NGNP white papers. These include criteria for evaluating the adequacy of DID, which contributes to the deterministic input to RIPB design decisions.

- There are a number of international precedents, including those from the U.S., IAEA, and the U.K. SAPs, and reflected in the NRC reviews of MHTGR, PRISM, and NGNP that support the view that LBE selection is best accomplished through an RIPB process which includes both deterministic and probabilistic inputs and preserves the principle of DID.

- A key challenge of any LBE selection process is to systematically define the IEs that are appropriate for the reactor design and the event sequences that realistically model the plant response to the IEs. This is necessary to derive the appropriate and limiting DBAs for the design. Simply removing inapplicable events from existing LWR events is not sufficient to define the events that are uniquely appropriate for a given design. 
- The LBE definition and selection process must be clear in making the distinction between IEs and event sequences. A given IE may result in different event sequences each having a different frequency of occurrence and level of severity in challenging the reactor safety defenses. Simply assuming the "worst active single failure" and concurrent loss of offsite power in combination with an IE does not necessarily yield the appropriate limiting accidents to define the licensing basis.

- As emphasized in NUREG-1860, PRA plays an important role in the identification and evaluation of uncertainties in the definition of event sequences and in the estimation of their frequencies and consequences. This information on sources of uncertainty and their influences on risk assessment are important inputs to establishing adequate consideration of the principles of DID in the selection and evaluation of LBEs and other RIPB decisions.

- In order to provide the technical basis for managing the risks of accidents that involve two or more reactors or radionuclide sources by preventing and mitigating such accidents, it is necessary to consider such accidents in the definition of LBEs and to measure frequencies on a per plant-year (multi-reactor module) basis, rather than a reactor-year basis.

- The development of frequency-consequence criteria for the LMP greatly benefits from the approach most recently advanced in the NGNP LBE white paper, as well as similar frequency-consequence criteria originally proposed by Farmer. Useful guidance is also available from NUREG-1860, the U.K. SAPs for event consequences, frequencies, and threshold for event evaluation.

- A key challenge in interpreting current U.S. regulations for limiting radiological exposures for normal operation and LBEs is the lack of explicit numerical criteria for categorizing events by expected frequency of occurrence. However, the classification of LBEs into AOOs, DBEs, and BDBEs based on expected frequency of occurrence is consistent with LBE classifications that were identified in this regulatory review including NGNP, PRISM, NUREG-1860, NUREG-2150, Yucca Mountain Pre-closure Safety Analysis, and the U.K. SAPs.

- There are a number of NRC criteria that explicitly constrain the risk and/or allowable consequences of radiological releases from nuclear power plants. These criteria include requirements to evaluate the adequacy of the proposed design of the plant against specific criteria. Some of the regulatory dose requirements are intended for evaluation of individual events, whereas others are expressed in terms of annual exposure limits, frequency of a given magnitude of release, and individual risks for the population in the vicinity of the plant site. The review of these criteria that was performed in the NGNP LBE white paper has been extended in this report and has yielded some new insights that are reflected in the proposed LBE selection approach as discussed in the next section.

- The above key points have been used to guide the development of the LBE selection process as discussed more fully in Section 4. 


\subsection{LMP LBE SELECTION APPROACH}

The regulatory bases reviewed in Section 3 of this report included two specific approaches for selecting LBEs for advanced non-LWRs, including the approach originally employed for the MHTGR, ${ }^{[44]}$ subsequently refined in the Exelon PBMR ${ }^{[51]}$ and NGNP ${ }^{[3]}$ projects, and then used as a basis for ANSI/ANS-53.1, ${ }^{[69]}$ the design standard for modular HTGRs. ${ }^{[4]}$ The other approach is described in NUREG-1860, which has parallels to the approach proposed for HTGRs and offers additional guidance on the desirable features of an LBE selection approach. In addition to these resources, the regulatory review identified many other documents that provide expectations and useful guidance in selecting an LBE selection approach for the LMP.

This section begins by listing the desirable attributes of an LBE selection process for advanced non-LWRs followed by reviews and observations from the supporting regulatory guidance. This review sets the stage for describing the proposed LBE selection process, which is described in the balance of this section together with examples that have been prepared based on two advanced non-LWR designs, including the MHTGR and PRISM.

\subsection{LBE Selection Process Attributes}

The desirable attributes of the LBE selection process for advanced non-LWRs, based on the lessons learned from the regulatory foundation in Section 3 and the objectives of the LMP, are described below.

\section{Systematic and Reproducible}

In principle, application of the process by different persons given the same inputs would yield a reasonably comparable set of LBEs. Any variations should only result from different states of knowledge that are fed into the process.

\section{Sufficiently Complete}

The LBE selection process should be capable of defining a sufficiently complete set of LBEs capable of defining the challenges to safety functions, radionuclide barriers, and protective strategies for emergency planning and accident management. In order to support the development of strategies to prevent and mitigate accidents involving multiple reactor modules and radionuclide sources, as occurred during the Fukushima Daiichi accident, and to enable the NRC review of design features responsible for implementing these strategies, the LBE selection process should address multi-module and multi-source accidents.

\section{Available for Timely Input to Design Decisions}

Importantly, the LBE selection process should recognize that design decisions that are impacted by LBE selection are made at an early stage of design and long before the licensing application is prepared. A key obstacle in limiting the progress in deploying advanced reactor technologies is the lack of predictability of licensing decisions. The LBE selection process should play an important role in supporting the optimization of the design with respect to safety. 


\section{Risk-Informed and Performance-Based}

The LBE selection process should be RIPB, consistent with LMP objectives. Risk-informed, as contrasted with risk-based, means that the process will include an appropriate balance of deterministic and probabilistic elements, and will be consistent with the principles of DID. Performance-based means that the process will include measurable and quantifiable performance metrics and will be consistent with NRC policies on the use of performance-based alternatives. The interfaces with other RIPB decisions such as SSC safety classification, definition of SSC requirements for capability and reliability, and implementation of DID strategies should be clearly defined.

\section{Reactor Technology-Inclusive}

Applying the process to different advanced non-LWRs having fundamentally different safety design approaches will yield an appropriate set of LBEs that are consistent and fairly defined across the different reactor technologies. Appropriate means that the LBEs are capable of identifying the unique safety issues for each technology. Specifically, the approach needs to support a consistent definition of LBEs for modular HTGRs, MSRs, and liquid-metal-cooled reactors using both thermal and fast neutron spectra and employing different safety design approaches. The LBE selection process should yield a uniform level of safety consistent with NRC Safety Goal and advanced reactor policies.

\section{Consistent with Applicable Regulatory Requirements}

The LBE selection process must account for current regulatory requirements with due regard to their applicability to advanced non-LWR technologies and associated safety design approaches.

These attributes are consistent with the objectives of the risk-informed approach to selecting LBEs documented in NUREG- 1860.

\subsection{Review of Previous LBE Selection Approaches}

The regulatory and precedent review summarized in Section 3 identified two approaches that have been proposed to select LBEs for advanced non-LWRs. One of these, which will be referred to as the NGNP approach, was originally developed and applied to the MHTGR and was subsequently refined in the pre-licensing interactions with the Exelon PBMR project, and further refined in the NGNP project. The second is that described in NUREG-1860. In addition to these, the Yucca Mountain pre-closure safety analysis ${ }^{[32]}$ may be considered as a third method for deriving LBEs using a RIPB process. In this case, the method is for a non-reactor facility governed by a different set of regulatory requirements because the NGNP approach has actually been applied to a conceptual non-LWR design that was supported by a PRA and subjected to review by the NRC and supporting national laboratories. The LBE selection process adopted for use in the LMP is developed starting with a review of that approach. The proposed LBE selection process is then developed from this review utilizing insights from the NUREG-1860 approach and the regulatory precedent reviews for proposing selected refinements. The goal of this review is to define an LBE selection process that has the attributes presented in the previous section. 
The NGNP LBE approach utilizes dose limits from NRC and EPA regulations and NRC Safety Goal QHOs to define frequency versus consequence criteria for evaluating the risks associated with LBEs as shown in Figure 4-1.

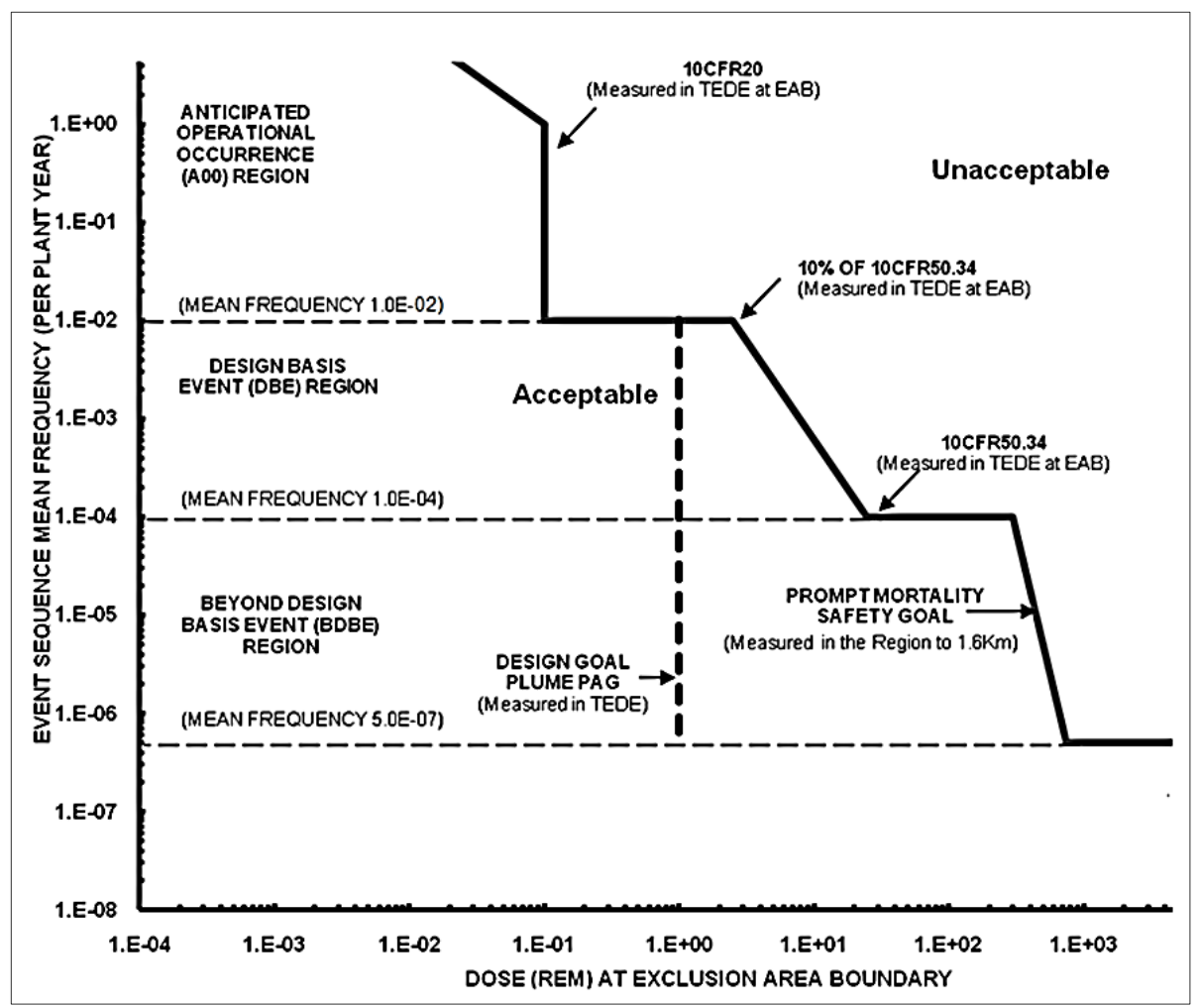

Figure 4-1. NGNP Frequency-Consequence Criteria

The key elements of the criteria are summarized as follows:

- LBEs are initially defined by accident families from the PRA results where each family has a similar IE, challenge to safety functions, plant response, and mechanistic source term for those families involving a release. LBEs are classified as AOOs with mean frequencies of $10^{-2} /$ plant-year or greater, DBEs with mean frequencies between $10^{-4} /$ plant-year and $10^{-2} /$ plant-year, and BDBEs with frequencies less than $10^{-4}$ /plant-year. The final category of LBEs, DBAs are derived from the DBEs using prescriptive rules to ensure that conservatively analyzed doses are within 10 CFR 50.34 dose limits without relying on any non-safety-related SSCs for mitigation.

- Estimates of the frequencies and consequences of LBEs include mean values and uncertainty intervals that account for sources of uncertainty in the estimation of event sequence frequencies and consequences.

- Many LBEs identify IEs and event sequences that challenge the PSFs but result in successful termination with no release of radioactive material and, hence, no offsite exposures. Understanding the plant design features responsible for accident prevention is an essential outcome of the LBE process. 
- Limitation of radiological exposures for individual AOOs that may involve a release to $100 \mathrm{mrem}$. For AOO frequencies of $1 /$ plant year and higher, an iso-risk profile is used to help define the F-C Target in the AOO region. For AOO frequencies from 1/ plant-year to $10^{-2}$ /plant year, the AOO dose limit is fixed at $100 \mathrm{mrem}$.

- The dose evaluation criteria for DBEs with a release range from $10 \%$ to $100 \%$ of the 10 CFR 50.34 dose limit of 25 rem for DBE frequencies that range from $10^{-2} /$ plant-year to $10^{-4}$ /plant-year, respectively.

- The dose evaluation criteria for BDBEs with a release range from 300 rem (low probability of early fatality) to 750 rem (high probability of early fatality) for BDBE frequencies that range from $10^{-4} /$ plant-year to $5 \times 10^{-7} /$ plant-year, respectively.

- Though not an NRC regulatory requirement, this Figure also shows the EPA Protective Action Guideline limit for sheltering at 1 rem to reflect an NGNP user requirement for reducing the size of the Emergency Planning Zone.

The NUREG-1860 LBE selection approach defines similar frequency versus consequence criteria, shown in Figure 4-2, to evaluate off normal event selection from a PRA. Both sets of frequency versus consequence criteria are developed based on somewhat differing interpretations of the then existing U.S. regulatory requirements that include annual limits on the radiological doses from normal operation, radiological dose evaluation criteria for the evaluation of AOOs and postulated accidents, and NRC QHOs.

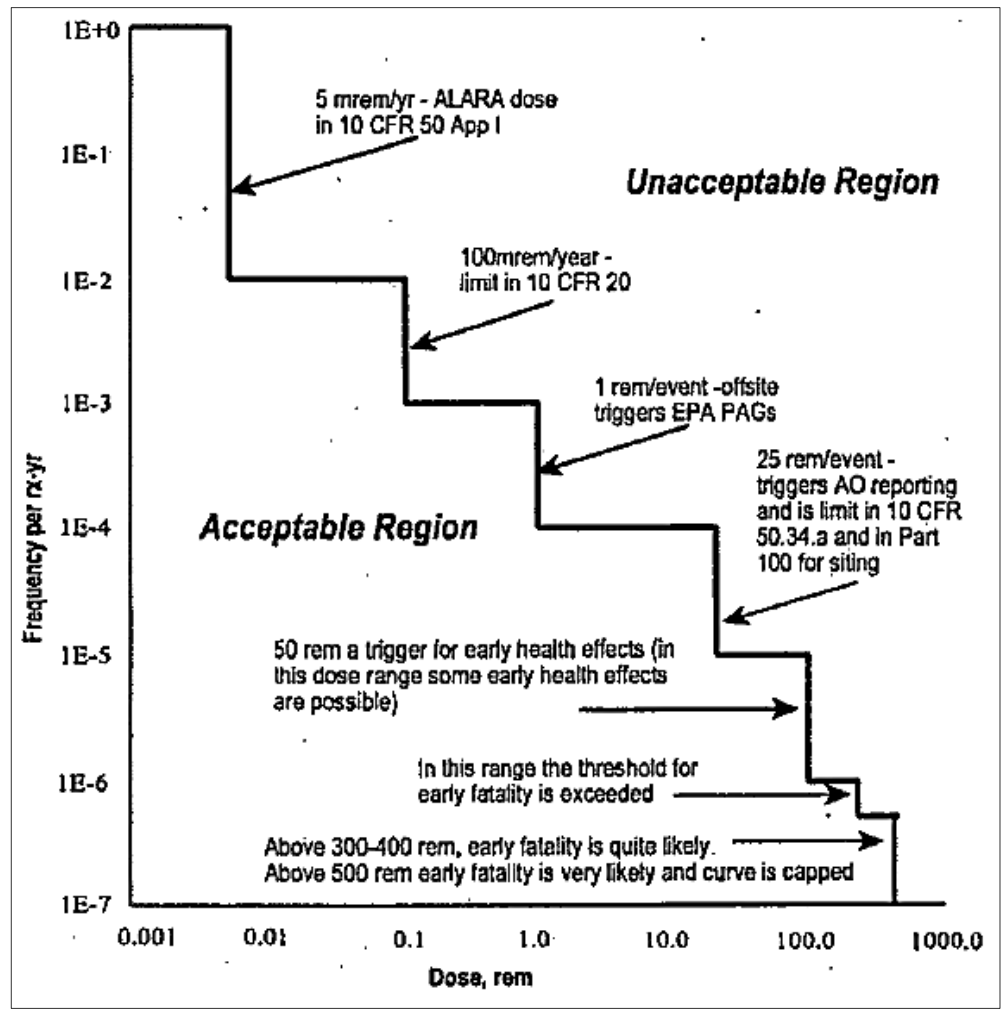

Figure 4-2. NUREG-1860 Frequency-Consequence Criteria 
Even though the two sets of criteria are based on the same underlying requirements, judgments are needed to associate the regulatory dose evaluation criteria to event frequencies because the U.S. regulatory requirements use qualitative statements in lieu of numerical frequency limits to describe the likelihoods of AOOs and postulated accidents to be evaluated against the dose limits. One key difference between the NGNP and NUREG-1860 criteria is that the former express frequencies on a per (multi-module) plant-year basis, whereas NUREG-1860 expresses frequency on a per reactor-year basis. By moving toward a quantitative measure of likelihood, the approach can be described as performance based. With the benefit of an updated review of more recent regulatory bases, some refinements to the selection of F-C Targets can be proposed.

The review of the NGNP and NUREG-1860 approaches for defining frequency-consequence criteria has identified three areas for improvement, including:

1. An alternative interpretation of $10 \mathrm{CFR} 20$ requirements for application to evaluating AOOs

2. Disadvantages of the use of a "staircase" shape for the frequency-dose profile

3. Need to consider the principle of risk aversion in setting the frequency versus dose evaluation boundaries for the AOO, DBE, and BDBE categories of LBEs

Each of these areas is discussed in the sections below.

\subsubsection{Interpretation of 10 CFR 20 and 10 CFR 50 Annual Exposure Limits}

Both of the NGNP and NUREG-1860 approaches defined frequency versus dose criteria for evaluating the frequencies and doses of individual LBEs. NGNP used 10 CFR 20 to define an iso-risk line (i.e., line of constant risk defined as the product of the frequency and dose) for frequencies greater than 1/plant-year, but as a fixed dose limit for events with frequencies between 1/plant-year and 10-2/plant-year. NUREG-1860 used the annual dose limits of 10 CFR 50 to limit doses from individual event sequences with frequencies between 1 and $10^{-2}$ /reactor-year, and the annual dose limits of 10 CFR 20 to limit doses of individual LBEs at frequencies between $10^{-2} /$ reactor-year and $10^{-3} /$ reactor-year. As noted in SRP Chapter 15.0, the doses from AOOs having a relatively low frequency of occurrence may exceed 10 CFR 20 so long as the risk, defined by the product of the frequency and consequence, is sufficiently low and other limits are not exceeded:

"If the risk of an event is defined as the product of the event's frequency of occurrence and its consequences, then the design of the plant should be such that all the AOOs and postulated accidents produce about the same level of risk (i.e., the risk is approximately constant across the spectrum of AOOs and postulated accidents). This is reflected in the GDC, which generally prohibit relatively frequent events (AOOs) from resulting in serious consequences, but allow the relatively rare events (postulated accidents) to produce more severe consequences."

However, according to SRP Chapter 15.0, the doses of lowest frequency AOOs for pressurized water reactors: 
"...shall not be sufficient to interrupt or restrict public use of those areas beyond the exclusion radius."

One metric that could be used to demonstrate that doses are not sufficient to restrict use beyond the EAB is the one rem EPA Protective Action Guide (PAG) limit for initiating offsite protective actions. This same part of the SRP states that for lower frequency AOOs for boiler water reactors,

“...the offsite release of radioactive material is limited to a small fraction of the guidelines of 10 CFR 100."

There are several places in the SRP, such as Chapter 15.0.3, where the concept of a "small fraction" is interpreted as $10 \%$. For example:

"A small fraction is defined as less than 10\% of the 10 CFR 50.34(a)(1) reference values, or 2.5 rem TEDE."

For the 10 CFR 50.34 dose limit of 25 rem, $10 \%$ equals $2.5 \mathrm{rem}$. From these acceptance criteria in Chapter 15 of the SRP, it is reasonable to permit the doses from the lower frequency AOOs to be as high as 1 rem (EPA PAG limit) to 2.5 rem (small fraction of 10 CFR 50.34 limit) for consistency with LWR AOO acceptance criteria.

The above statements from SRP Chapter 15 suggest that both the NGNP and NUREG-1860 frequency-consequence criteria are too conservative in interpreting the 10 CFR 20 annual dose limits as to not exceed criteria for individual LBEs. The conservatism stems from applying annual dose limits to individual events. This insight is used to propose alternative frequencyconsequence criteria in Section 4.3.

\subsubsection{Staircase Discontinuity Issue}

A second issue that was identified from reviewing the NGNP and NUREG- $1860^{*}$ frequency versus consequence criteria is referred to here as the "staircase discontinuity" issue. This issue is illustrated in Figure 4-3 using the NGNP criteria as an example; however, both sets of criteria are subject to this issue.

\footnotetext{
"The risk targets in the U.K. SAPs also obey a "staircase" shape when plotted on a log frequency versus log consequence graph.
} 


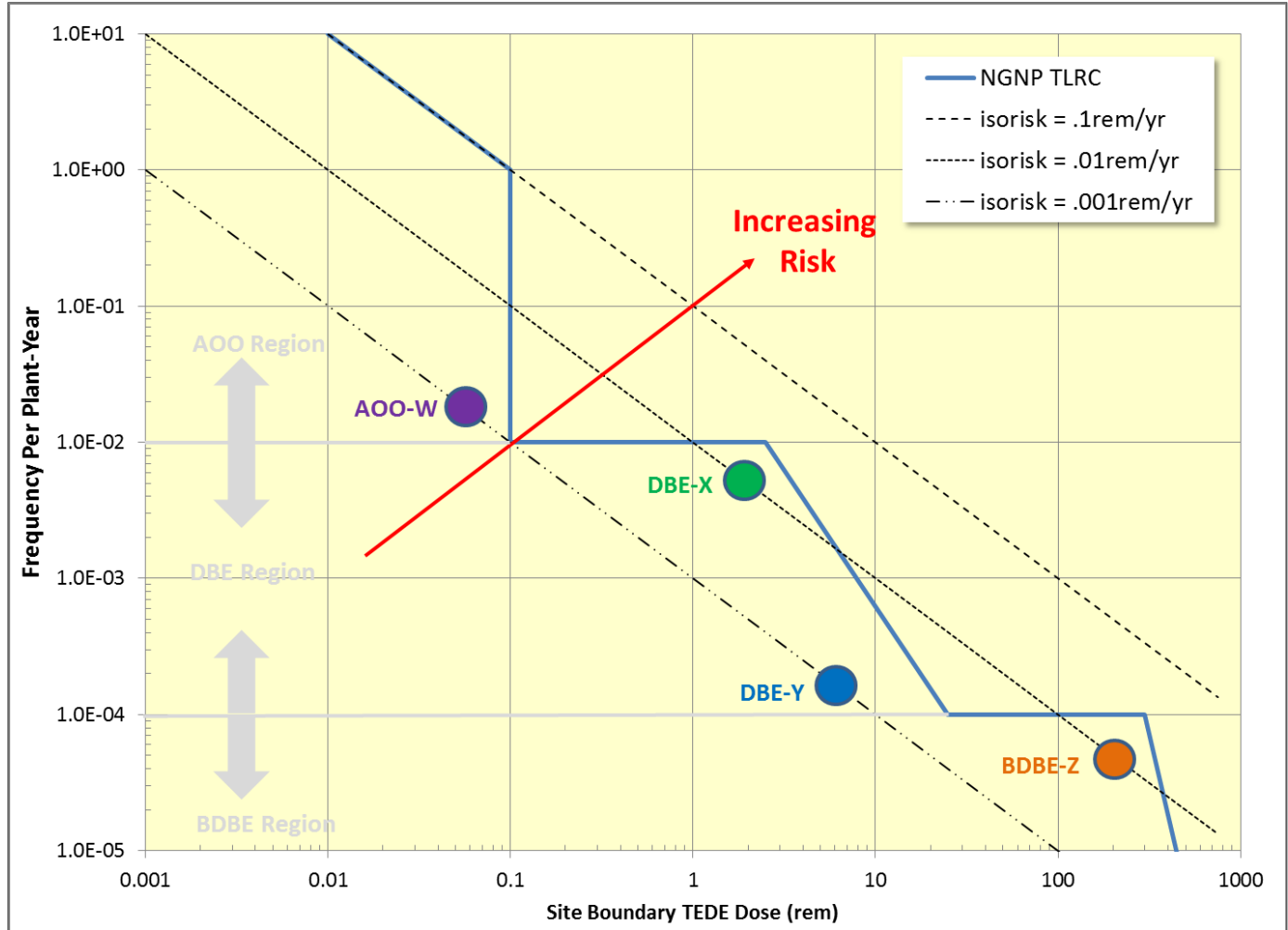

Figure 4-3. Part of NGNP Frequency-Consequence Criteria Illustrating Staircase Issue

The NUREG-1860 version of the criteria is essentially a succession of smaller staircase steps. The NGNP criteria are based on the interpretation that the 10 CFR 20 annual dose limit should be used as the limit on the dose from individual AOOs with frequencies from 1/plant-year to $10^{-2} /$ plant-year. This lower AOO region frequency of $10^{-2} /$ plant-year is also the upper limit of the DBE region in the NGNP criteria. The NGNP dose criteria for the DBE region range from $10 \%$ of the 10 CFR 50.34 limit at the top end of the frequency range to $100 \%$ of the same dose limit at the bottom end of the DBE frequency range of $10^{-4} /$ plant-year.

There is another staircase in the transition from the $\mathrm{DBE}$ region to the $\mathrm{BDBE}$ region in the NGNP criteria. The lower limit of the BDBE region is set at a frequency $\left(5 \times 10^{-7} /\right.$ plant-year $)$ at which it can be assured that the QHOs for early health effects can be satisfied independent of the level of consequences. The dose assigned to the criteria at this frequency is 750 rem TEDE, which is associated with a high probability of death for a person located at the EAB. The dose assignment at the $5 \times 10^{-7}$ frequency level is already a very conservative representation of the QHOs because the early fatality QHO is based on the average individual risk over the entire area between the EAB and 1 mile out from the EAB. Considering the fact that the dose drops off with a distance across the 1 mile "doughnut" and also drops off very rapidly as one moves off the center-line of the direction of the release plume, the average individual risk would be much less than the QHO for an accident at much higher frequencies than $5 \times 10^{-7}$, even if the EAB dose at the center line of plume would approach $750 \mathrm{rem}$. A task is included in the NGNP approach to confirm that QHOs have been met based on an integrated assessment of the individual risk in the area surrounding the plant accounting for all the LBEs. 
The combination of assumptions used to draw the NGNP frequency versus consequence curve from $1 /$ plant-year to $10^{-2}$ /plant-year creates an undesirable property that the "risk" as defined as the product of the frequency and dose is allowed to increase as the LBE frequencies change from the lower end of the AOO region in the vicinity of AOO labeled "AOO-W" to the upper end of the DBE region in the vicinity of "DBE-X." In these examples, the risk of DBE-X is an order of magnitude greater than that for AOO-W. There is a similar problem when comparing the risks between "DBE-Y" and "BDBE-Z" in which case BDBE-Z, having a somewhat smaller frequency, has a higher risk than DBE-Y, while both are on the acceptable side of the frequencyconsequence criteria. In both of these examples, the frequency-consequence criteria permit higher risks with small reductions in frequency at the transitions across the staircase steps. NUREG-1860 also has these staircase discontinuities; however, there are more steps in those frequency-consequence criteria which somewhat alleviate the concern.

This staircase issue creates an implementation issue to be avoided in designing and licensing a new reactor. As the design matures and the PRA is updated to incorporate plant design changes and refinements to requirements, the LBE frequencies and consequences are subject to change and likely will change. In addition, the LBE frequency and consequence estimates are not points but cover a range of values within the uncertainty bounds that may overlap the frequency cutoffs for each LBE region. It is problematic to permit large changes in allowable risk when transitioning from one LBE region to another, which any staircase criteria would suggest. The uncertainties in frequency and consequence estimates, as well as the changes in the estimates that would be expected from successive PRA updates during the evolution of design, require more continuous behavior in the risk-acceptance criteria.

The NGNP frequency-consequence criteria has one "knee" in the curve at the upper end of the BDBE region established by the upper frequency limit of $10^{-4} /$ plant-year and a dose limit of $300 \mathrm{rem}$, which corresponds to a probability of fatality due to prompt radiation syndrome of 0.005 (i.e., the lower bound of the probability of death versus exposure curve). Using 25 rem at the lower end of the DBE region and 300 rem where the BDBE region starts and DBE region leaves off is a big increase and requires good justification. A regulatory basis for this point at the knee does not exist. The derivation of this knee appears to be based on a mixture of accident frequencies and conditional probability of a certain consequence from a release. The probability of fatality from a given exposure is an important factor to consider in estimating the individual risk of fatality from a given release. However, the probability of fatality curve has been used here as a basis for establishing a relationship between event frequencies and acceptable exposures for events at a given frequency. This then yields a risk, defined as the product of the frequency and dose at the upper end of the BDBE region, which is much higher than that at the lower end of the BDBE region and at the lower end of the AOO region. Hence, it does not appear that a good basis exists for establishing the upper knee of the criteria in the BDBE region at 300 rem and $10^{-4}$.

To avoid these issues, it is desirable that acceptable risk levels should not be allowed to increase when transitioning from the AOO region to the DBE region or when transitioning from the DBE region to the $\mathrm{BDBE}$ region. This consideration leads to some proposed refinements to the frequency-consequence criteria as described in Section 4.3. 


\subsubsection{Plant-Year Versus Reactor-Year Frequency Basis}

A key difference between the NGNP and NUREG-1860 frequency-consequence criteria is the different frequency bases that are used. NGNP defines the frequency basis on a per plant-year basis where a plant may be comprised of two or more reactor modules in order to address LBEs that may involve releases from two or more reactor modules or sources of radioactive material. This approach addresses the increased likelihood in the frequency of single-unit events that occur on each reactor modules independently and enables a meaningful comparison of the frequencies of single and multi-module events, as well as events from a common radionuclide source such as fuel storage facility.

NUREG-1860 retains the traditional PRA approach that has been used for operating LWRs where LBEs derived from PRAs are addressed for each reactor on a one-reactor-at-a-time basis, which leads to expressing frequencies on a per reactor-year basis. The NUREG-1860 approach makes it problematic to compare LBEs that involve single and multiple reactor source terms and fails to measure the increased likelihood of independent events occurring on each module independently.

Lessons learned from the Fukushima Daiichi accident, as exemplified in the Near-Term Task Force report, ${ }^{[28]}$ cannot be effectively addressed by an evaluation that is done on a one-reactor-ata-time basis. The need to address both single reactor and multiple reactor events was highlighted in several other regulatory precedents reviewed in Section 3, including Chapter 19 of the SRP, SECY 2003-0047, and several other references. For example, it is noted that the U.K. SAPs include frequency-consequence criteria both on a per reactor-year basis for the purpose of evaluating the generic design assessments similar to the U.S. design certifications, and on a per site-year basis for addressing the integrated risks for an entire site.

The LMP prefers the NGNP approach because it provides a basis to address LBEs for a multimodule plant design. Addressing the integrated risks of an entire site, which may include other plants not within the scope of an advanced non-LWR license application, is considered beyond the scope of this project. Hence, the per plant-year frequency basis is selected for use in the LMP frequency-consequence criteria.

\subsubsection{Risk Aversion Considerations}

The next topic addressed in the review of proposed frequency versus consequence criteria is the principle of risk aversion. In application of this principle, risk targets of low frequency events that may have large consequences are set to lower risk criteria than those for higher frequency events which are expected to have lower consequences. One of the first proposals for numerical frequency versus consequence criteria was made by Reginald Farmer of the U.K., ${ }^{[68]}$ who is recognized as the father of PRAs applied to assess the risks of reactor accidents. His proposed limit lines are illustrated in Figure 4-4 and are expressed in terms of accident frequency versus quantity of release of the key radionuclide I-131. 


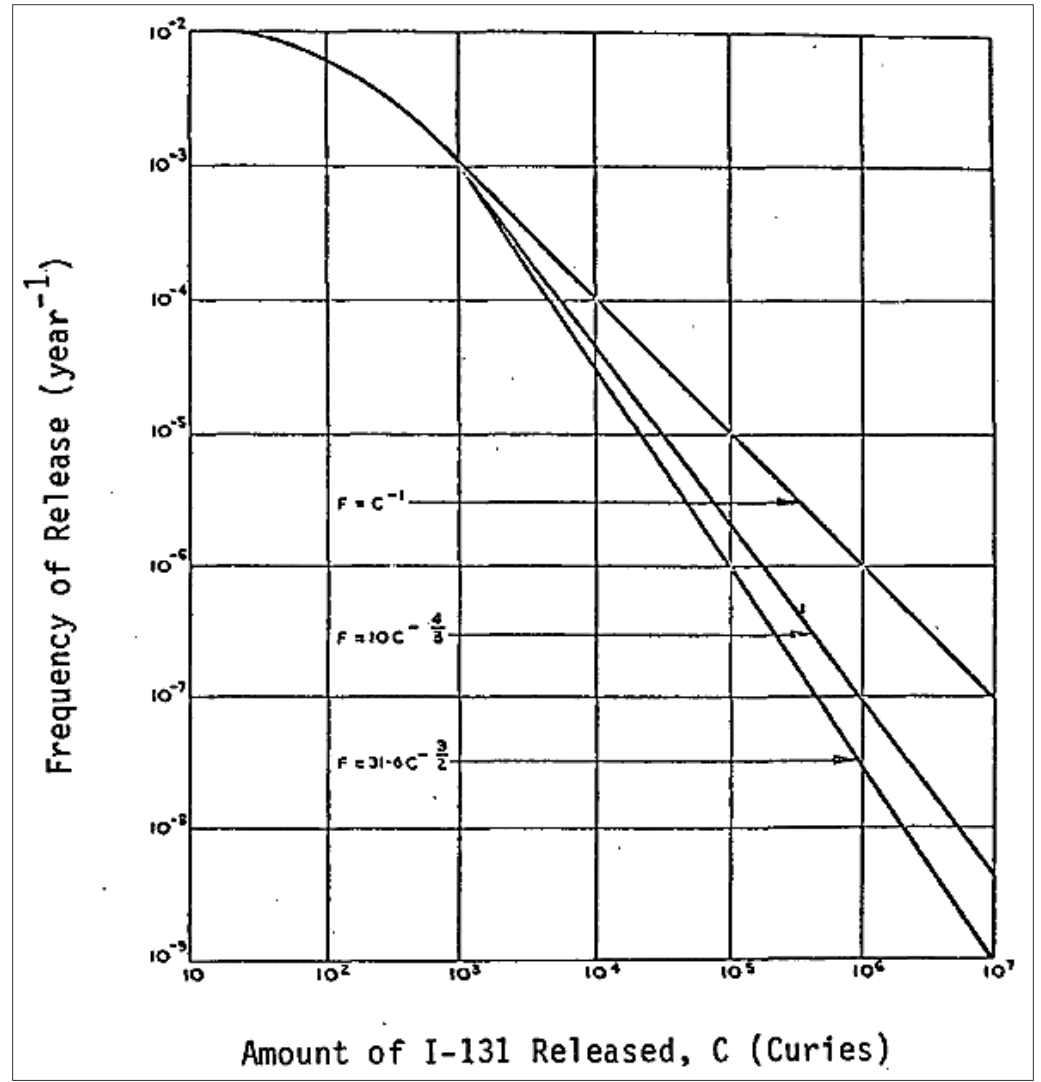

Figure 4-4. Frequency Versus Consequence Limit Line Proposed by Farmer ${ }^{[68]}$

This risk metric was used in the early days of nuclear power in the U.K. to address the question, "How safe is safe enough?" The top limit line follows an iso-risk contour at frequencies below $10^{-3} /$ year that when plotted on log-log paper is shown as a straight line with logarithmic slope of -1 . The iso-risk contour is neutral with respect to the risk aversion principle, which is applied in the lower two curves having steeper logarithmic slopes of -1.33 and -1.5 , respectively, at frequencies below about $10^{-3} /$ year. The curve having the greatest allowance for risk aversion is the lowest curve with logarithmic slope of -1.5 , which is the acceptance criterion proposed by Farmer.

The principle of risk aversion is considered in the formulation of revised frequency-consequence criteria as developed in Section 4.3.

\subsubsection{Definition of LBE Categories}

The NGNP approach for the definition of LBE frequency categories of AOO, DBE, and BDBE is somewhat different from the approach used in NUREG-1860. The latter reference uses the traditional PRA frequency metric of events/reactor-year, whereas NGNP uses frequency/plantyear where a plant may be comprised of multiple reactor modules as discussed in the NGNP white paper. NUREG-1860 classifies events as "frequent" with frequencies greater than $10^{-2}$ /reactor-year, "infrequent" with frequencies between $10^{-5}$ /reactor-year and $10^{-2} /$ reactor-year, and "rare" with frequencies less than $10^{-5} /$ reactor-year. NUREG-1860 does not address the multi-module risk issue. However, considering that modular reactor plants with as many as 
12 modules have been proposed, the frequency classes proposed in the respective references are comparable, as will be shown in the next section. As noted in ANS/ANSI-53.1, "Nuclear Safety Design Process for the Design of Modular Helium-Cooled Reactor Plants:"
"The adoption of the DBE region's lower frequency limit of $1 \times 10^{-4}$ (1E-04) per plant- year is appropriate because it is applied on a per-plant basis and accounts for possible multiple MHR modules. In addition, the expression of the frequency metric on a per plant-year basis enables the assessment to include event sequences involving only one or multiple reactor module source terms and thereby provides a more complete risk assessment as compared with the approach of analyzing each reactor module on an independent reactor-year basis."

As noted in the SRM to SECY 90-16, the NRC endorsed a core damage frequency (CDF) goal of $10^{-4} /$ reactor-year for advanced reactors. However, even for advanced reactors, core damage events are regarded as beyond design basis accidents. Hence, the selection of a lower limit of the DBE region of $10^{-4} /$ plant-year, where a plant may be comprised of multiple reactor modules, is conservative relative to the NRC's advanced reactor CDF goal, which is only for a single reactor.

The LMP has chosen the NGNP LBE frequency criteria for differentiating between AOOs, DBEs, and BDBEs. This selection is made to support the capability to address multi-module events and events that may involve two or more sources of radioactive material.

\subsubsection{Risk Evaluation of LBEs and Integrated Risk Assessment}

The approach to evaluating risk significance of LBEs accounts for estimates of frequencies and site boundary doses, including their mean values and uncertainty distribution upper and lower percentiles.

Consistent with the NGNP approach, in classifying individual LBEs into the correct bins based on frequency in the LMP approach, when the mean frequencies are in the AOO region but the lower $5 \%$ of the frequency is in the DBE region, the LBE shall be evaluated both as an AOO and a DBE. If the mean frequency is less than $10^{-4} /$ plant-year and the upper $95^{\text {th }}$ percentile frequency is in the DBE region, the LBE is also evaluated as a DBE. Hence, when the uncertainty band on the frequencies straddles an LBE frequency criterion that separates AOOs from DBEs and BDBEs, the LBE is evaluated using the criteria in both regions.

Further, in addition to the risk evaluation of individual LBEs, the integrated risks considering the total from all the LBEs is also considered in the NGNP approach. Although shown on the F-C graph, which will be referred to in the rest of this report as the F-C Target, developer and users need to keep in mind that the factors are related to the aggregate risks from all LBEs and may not be appropriate for assessing individual LBEs. The integrated risks could, in theory, result in a need to consider further plant improvements or result in limitations related to siting, emergency planning, or operational programs beyond those identified by assessing individual LBEs. For this purpose, the risk metrics to be used are those for comparison against the two NRC QHOs. The QHOs are defined in the NRC Safety Goal Policy Statement. As noted in the previous section, that policy statement also has a performance goal for maintaining the frequency of a 
large release below $10^{-6}$ year. It is recognized that there have been some differing views as to whether the NRC Safety Goals and the associated QHOs and performance goals should be applied on a per site-year or a reactor-year basis.

As noted previously, the annual dose limits in 10 CFR 20 have been used to develop the F-C Target for evaluating individual LBEs in the AOO region. The annual dose limits in 10 CFR 20 also imply a limit on the integrated risks of LBEs that supplement those derived from the Safety Goal Policy and serve to limit the cumulative risks of LBEs in the high frequency and low consequence part of the risk spectrum. Although it is reasonable to use 10 CFR 20 annual dose limits as a basis for developing the F-C Target for higher frequency LBEs, as has been done for both NGNP and NUREG-1860, just meeting the criteria for events evaluated individually does not necessary satisfy the integrated annual doses that are limited in 10 CFR 20. Hence, this additional integrated risk metric has been added to confirm that 10 CFR 20 is met for the summation of all the LBEs.

In view of these considerations the LMP has chosen to retain the two QHO goals used in the NGNP approach for evaluating the integrated risks and add one additional goal to address the 10 CFR 20 annual dose limits:

- The total frequency of exceeding a site boundary dose of 100 mrem shall not exceed 1/plant-year to ensure that the annual exposure limits in 10 CFR 20 are not exceeded.

- The average individual risk of early fatality within the area 1 mile of the EAB shall not exceed $5 \times 10^{-7}$ /plant-year to ensure that the NRC Safety Goal QHO for early fatality risk is met.

- The average individual risk of latent cancer fatalities within the area 10 miles of the EAB shall not exceed $2 \times 10^{-6} /$ plant-year to ensure that the NRC Safety Goal QHO for latent cancer fatality risk is met.

The combination of these three cumulative risk targets covers both the high frequency and low frequency ends of the event spectrum. It is noted that the above cumulative risk targets are in addition to the design goal to maintain the risks for the individual LBEs within the F-C Target and are applied on a per plant-year basis so that the integrated risks of accidents involving single and multiple reactor modules and sources of radioactive material are included. The application of these integrated risk criteria to a plant which may be comprised of multiple reactor modules, rather than to individual reactors is consistent with an ACRS recommendation on treatment of integrated risks for advanced non-LWR designs, which states: ${ }^{[73]}$

"The Quantitative Health Objectives (QHOs) apply to the site as a whole. The sum of the contributions from each reactor on the site to acute and latent fatalities should be bounded by the QHOs."

\subsubsection{Summary of Review Findings}

The take-away lessons from this part of the LBE approach review are as follows: 
- Both the NUREG-1860 and NGNP approaches to LBE selection appear to meet the LBE selection attributes listed in Section 4.1. The major elements of both approaches are comparable and are judged to be capable of providing the desired characteristics of being risk-informed, performance-based, reproducible, and capable of identifying a sufficiently complete set of limiting reactor specific LBEs.

- The interpretation of 10 CFR 20 annual exposure criteria as a risk limit, rather than a dose limit for individual events, is judged to be more consistent with the intent of the current regulatory requirements as discussed in SRP Chapter 15.0.

- For consistency with requirements for lower frequency AOO events in SRP 15.0, which state that doses from lower frequency AOOs should not impact offsite activities, the radiological exposures for lower frequency AOOs should not exceed the EPA PAG limits for triggering offsite protective actions. Limiting lower frequency AOO events to $1 \mathrm{rem}$ TEDE at the EAB would satisfy this requirement.

- There are disadvantages to having staircase discontinuities in the frequency versus dose criteria. Otherwise, small reductions in LBE frequencies would yield higher risk targets as the frequency thresholds at the stair steps are crossed. Small changes in LBE frequencies in any part of the frequency-consequence spectrum should be evaluated against small changes in dose criteria, and not against large step changes in criteria.

- In order to apply the principle of risk aversion, it is appropriate to accept lower criteria on risks for lower frequency accidents in the DBE and BDBE regions which have the potential for higher consequences.

- The NGNP approach to defining LBE frequency categories of AOO, DBE, and BDBE on the basis of frequency per plant-year is preferred over the NUREG-1860 method which uses a per reactor-year frequency basis in order to make meaningful comparisons of risks between event sequences involving single and multiple reactor modules and radionuclide sources.

These lessons are reflected in proposed F-C Targets as described in the next section.

\subsection{LMP Revisions to NGNP Frequency-Consequence Evaluation Criteria}

Based on the insights from the review of existing criteria, the LMP proposes to use a set of frequency-consequence criteria that adopt the NGNP criteria as a basis with some refinements to address the review insights from the previous section. The criteria proposed in NUREG-1860 are used as guidance and as a sanity check to compare against the proposed criteria after some adjustments that attempt to reconcile the differences in frequency bases. The reason for starting with the NGNP approach is that this approach is more consistent with LMP objectives. The following elements of the NGNP approach are viewed as more consistent with the LBE attributes discussed in Section 4.1, namely:

- The classification of LBEs into distinct categories of AOOs, DBEs, and BDBEs lead to systematic identification of DBAs and other events used to formulate licensing requirements. The NGNP approach provides a clean interface with the results of the PRA 
and prescriptive inputs to the deterministic safety analysis. This approach addresses the needs of the designer and supports the attribute of predictability of the licensing process.

- The NGNP approach to selection of LBEs has its origins in the MHTGR design and licensing approach. It provides benefits to the full plant application of RIPB insights that supports LBE selection, SSC safety classification, and derivation of reactor specific design criteria.

- The frequency basis of events/plant-year facilitates application to advanced non-LWR plant designs using a modular reactor approach. This provides the designer with capabilities to define LBEs and develop design strategies to prevent and mitigate accidents involving both single and multiple reactor modules and non-core radionuclide sources.

- The frequency-consequence evaluation correlation proposed for the LMP is referred to in the rest of this report as the F-C Target and is shown in Figure 3 5. The F-C Target in this figure is based on the following inputs referred to as Top Level Safety Targets:

- The regions of the graph separated by the frequency-dose evaluation line are identified as "Increasing Risk" rather than "unacceptable," and "Decreasing Risk" rather than "acceptable" to emphasize that the purpose of criteria is to evaluate the risk significance of individual LBEs and to recognize that risk evaluations are not performed on a passfail basis in contrast with deterministic safety evaluation criteria. This change is consistent with NRC risk-informed policies such as those expressed in RG 1.174 in which risk is not "accepted" but rather evaluated for risk significance.

- The evaluation line doses for high frequency AOOs down to a frequency of $10^{-1} /$ plantyear are based on an iso-risk profile defined by the annual exposure limits of 10 CFR 20, or $100 \mathrm{rem} /$ plant-year.

$\circ$ The doses for AOOs at frequencies less than $10^{-1} /$ plant-year are capped at 1 rem corresponding with the PAG limits. This limit invokes the principle that AOOs should not impact offsite activities. However, other factors may prove to be more restrictive than the F-C Target because plant responses to AOOs are expected to limit challenges to fission product barriers and support resumption of operation following possible plant repairs or other corrective actions. Indeed, it is expected that many LBEs will not be associated with any release of radioactive material and the identification of plant capabilities to prevent such releases is a factor considered in the formulation of SSC safety classification and performance targets as discussed more fully in the companion LMP report on SSC safety classification.

- The F-C Target for DBEs range from 1 rem at $10^{-2} /$ plant-year to $25 \mathrm{rem}$ at $10^{-4} /$ plantyear. Considering this is a frequency per plant-year which may be applied to multimodule plants, the $10^{-4}$ /plant-year target for the bottom of the DBE range is more conservative than the NRC CDF goal for advanced reactors at $10^{-4} /$ reactor-year. ${ }^{[39]}$ This limits the lowest frequency DBEs to the limits in 10 CFR 50.34 and provides continuity to the lower end of the AOO criteria. A straight line on the log-log plot connects these criteria. 
- The F-C Target for the BDBEs range from 25 rem at $10^{-4} /$ plant-year to 750 rem at $5 \times 10^{-7} /$ plant year to ensure that the QHO for early health effects is not exceeded for individual BDBEs consistent with the NGNP F-C Target. The question of meeting the QHOs for the integrated risks over all the LBEs is addressed using separate cumulative risk targets described later in this report.

- The frequency-dose anchor points used to define the shape of the curve are indicated in Figure 4-5. The lines between the anchor points are straight lines on a log frequency verses log dose graph.

- In consideration of the risk aversion principle, the logarithmic slope of the curve in the $\mathrm{DBE}$ and BDBE regions exceeds -1.5 which corresponds to the most conservative limit-line proposed by Farmer to address risk aversion as shown in Figure 4-4.

- More details on the basis for the selection of the F-C target anchor points and the frequency boundaries of the LBE categories are found in Appendix B.

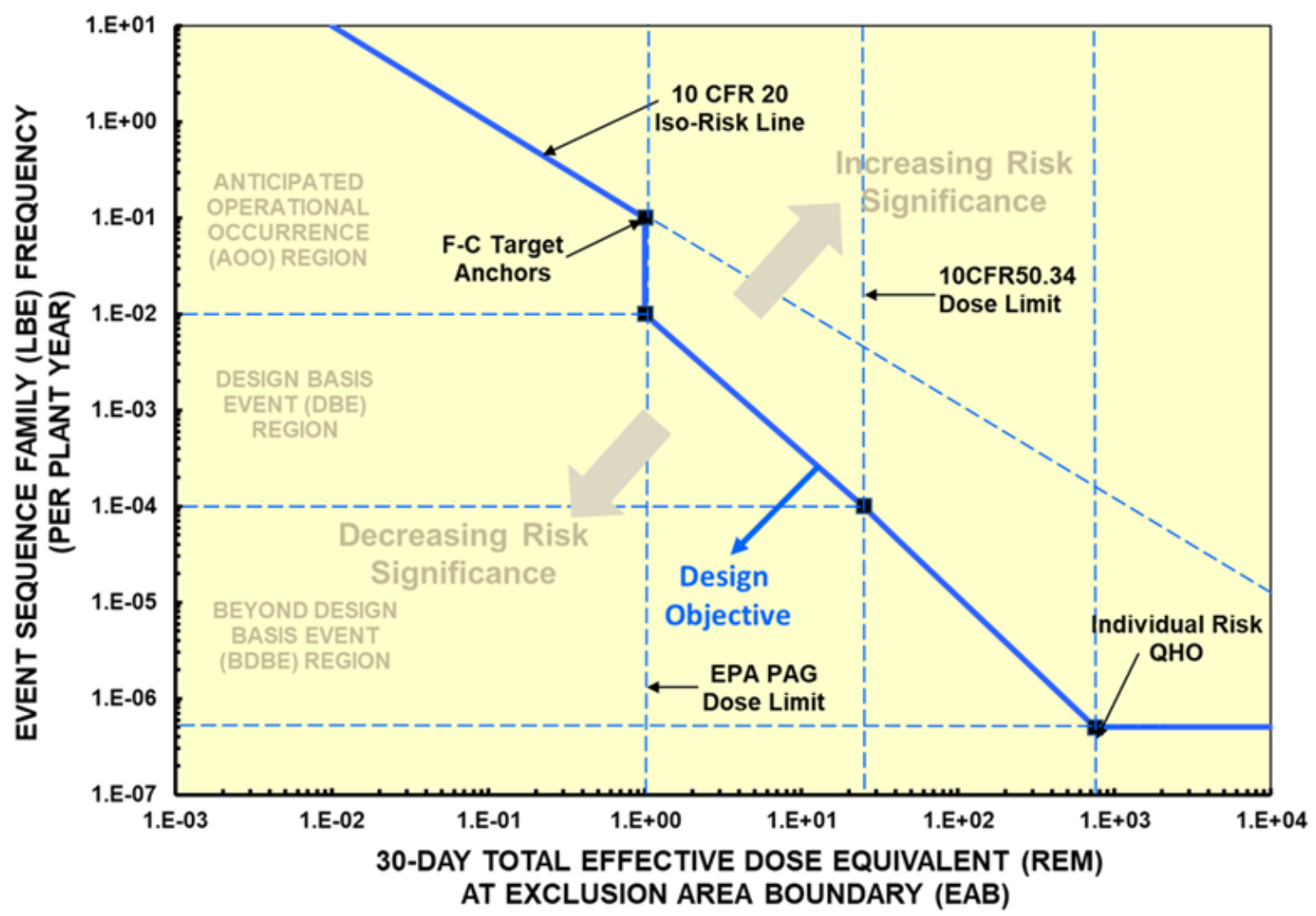

Figure 4-5. Frequency-Consequence Evaluation Criteria Proposed for LMP

The F-C Target in Figure 4-5 address the issues raised in the criteria review discussed in the previous section. This formulation eliminates the staircase issue. Across the entire spectrum the risk defined as the product of the frequency and consequence is not permitted to increase as the frequency decreases. In addition, the principle of risk aversion is applied at frequencies below $10^{-1} /$ plant-year. The logarithmic slope of the criteria between $10^{-2} /$ plant-year to $5 \times 10^{-7} /$ plant-year 
is about -1.5 , consistent with Farmer's more restrictive limit line, which may be regarded as the first proposal for an F-C Target for nuclear power plants.

While interpreting the 10 CFR 20 annual exposure limits of $100 \mathrm{mrem} / \mathrm{year}$, it is recognized that the proposed use of this criteria in developing the F-C Target is to be applied to individual LBEs. In order to ensure that the cumulative releases considering all the LBEs do not exceed this limit, the LMP LBE proposes to add a task not included in the NGNP LBE paper to ensure that the integrated risks summed over all the LBEs do not exceed $100 \mathrm{mrem} / \mathrm{year}$. This limit serves to control the risks in the high frequency low consequence end of the event spectrum noting that the NRC Safety Goal QHO cumulative risk targets are most effective in controlling the low frequency, high consequence end of the spectrum. As noted, the proposed LBE approach also retains the NGNP task of performing an integrated assessment over all the LBEs to ensure that NRC Safety Goal QHOs for both early and latent health effects are met.

A comparison of the proposed criteria and the NGNP criteria is shown in Figure 4-6. As seen in this figure, the LMP proposed criteria are less restrictive in the AOO region taking advantage of more up to date interpretations of the existing SRP acceptance criteria for AOOs in LWRs. However, the proposed criteria are somewhat more restrictive in the DBE and BDBE regions. Based on examples from HTGR and sodium fast reactor PRAs presented in this report and in a companion report on PRA development, it is expected that this will not cause any issues with advanced reactor designs. This modification primarily results from resolving the "staircase" discontinuity issues identified in this report with the NGNP criteria.

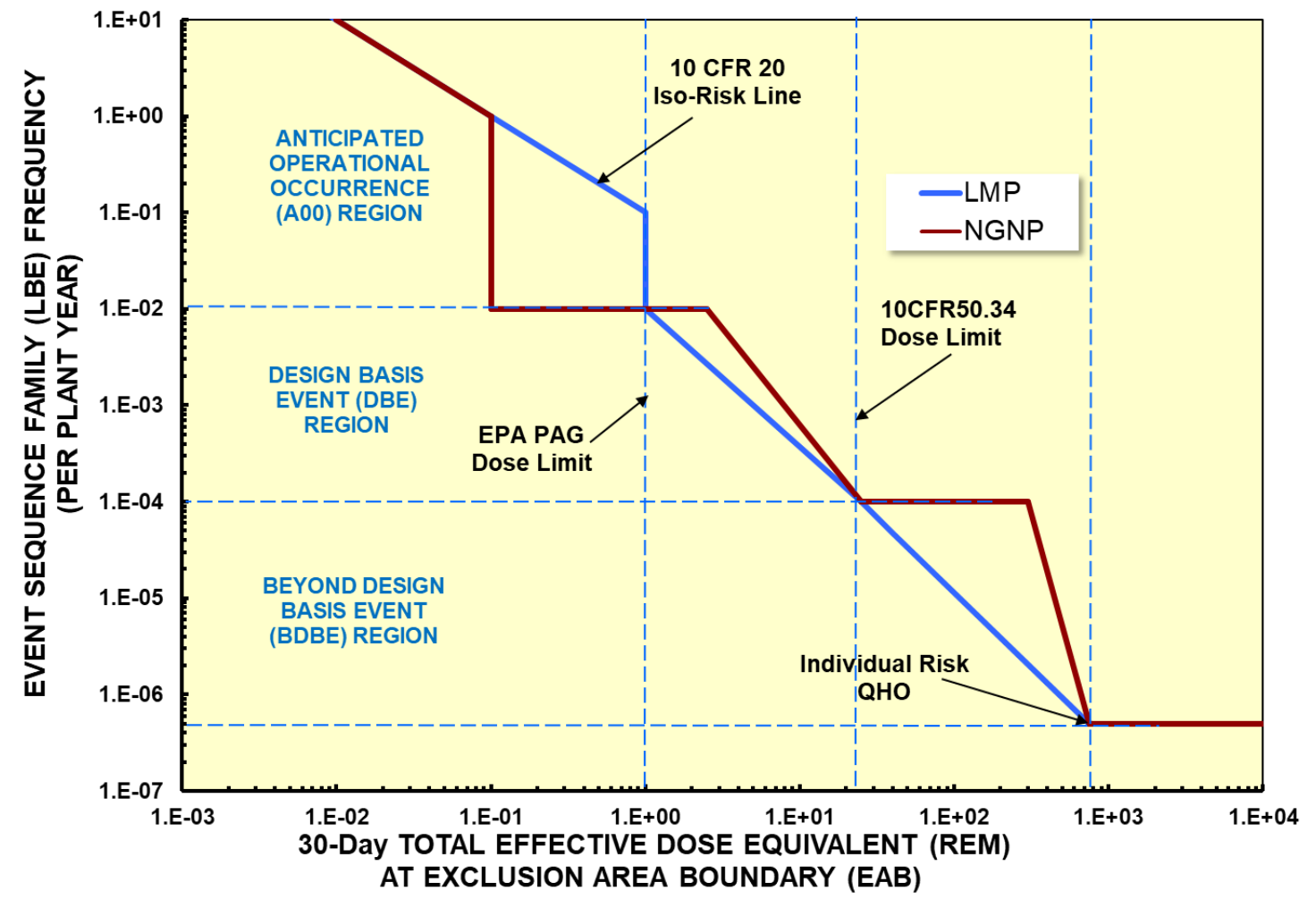

Figure 4-6. Comparison of LMP and NGNP Frequency-Consequence Criteria 
A similar comparison is made in Figure 4-7, in this case to contrast the LMP and the NUREG1860 criteria. The NUREG-1860 criteria are expressed on a per reactor-year basis. In order to compare against the LMP criteria, which are for a multi-module plant, three versions of the NUREG-1860 are shown, one for a 1-module plant, one for a 4-module plant, and a third for a 12-module plant. The 4-module and 12-module versions are obtained by simply scaling the frequencies which is recognized to be appropriate only for event sequences affecting a single module. As seen in this comparison, the NUREG-1860 criteria are much more restrictive for high frequency events with frequencies above 10-2/plant-year. However, for frequencies below about 10-3/plant-year the respective criteria are quite comparable. The LMP criteria for the high-frequency range are judged to be more consistent with the most recent update of the SRP Chapter 15 for criteria used to evaluate AOOs in current generation LWRs. The NUREG-1860 criteria are based in part on the use of annual exposure limits from 10 CFR 50 Appendix I. However, such criteria are not used in the SRP Chapter 15.0 for evaluating exposures for individual AOOs for LWRs. For the lower frequency range, the primary difference between the two sets of criteria is seen to be due to the staircase effects. On balance, these comparisons provide a useful sanity check on the reasonableness of the proposed criteria for the frequencies and consequences of LBEs for the LMP.

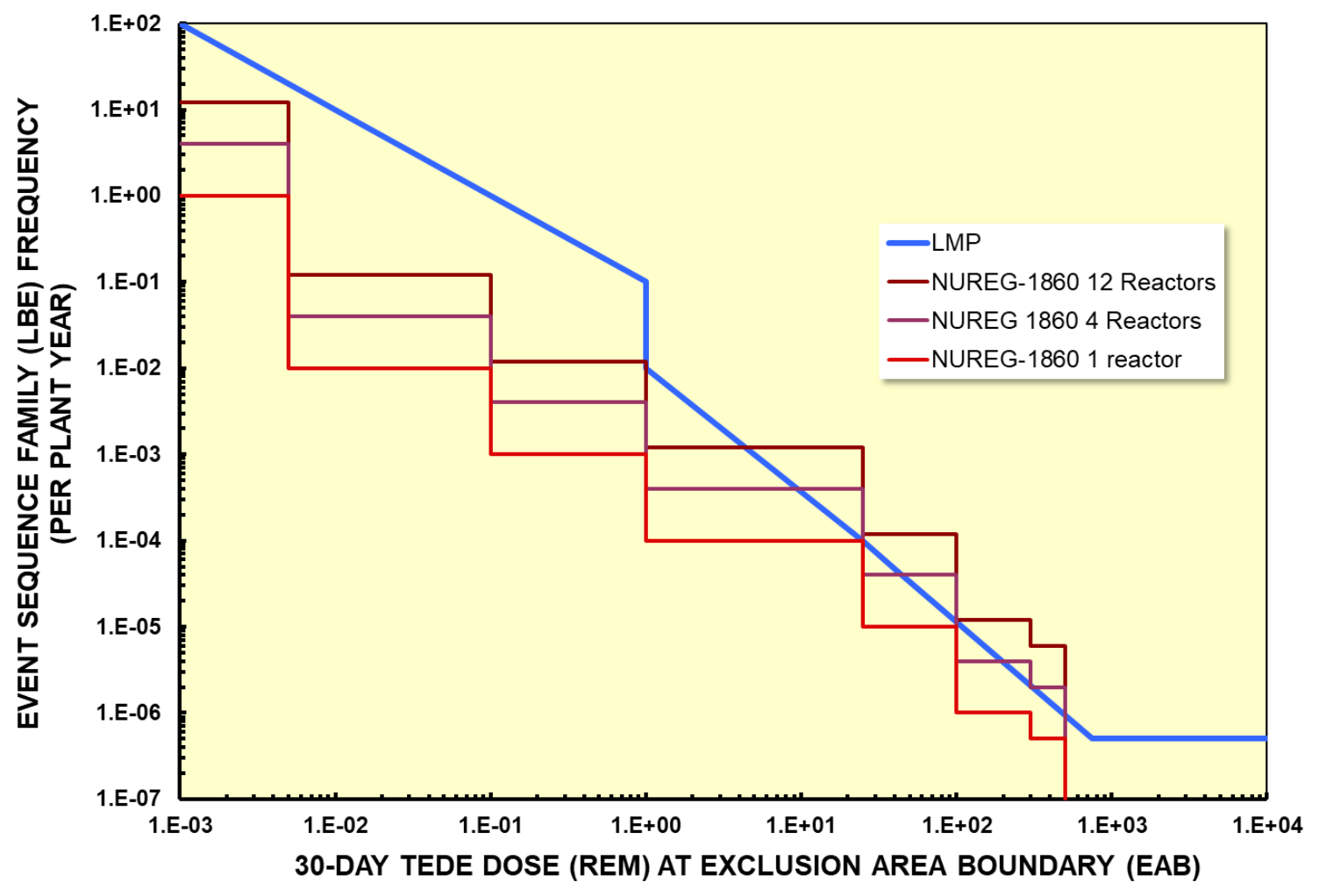

Figure 4-7. Comparison of LMP and NUREG-1860 Frequency-Consequence Criteria 


\subsection{LMP LBE Selection Process}

\subsubsection{LBE Selection Process Overview}

The design of advanced non-LWRs will be developed using a systematic, top-down TI-RIPB approach to meeting regulatory and end-user requirements that achieves the LBE selection attributes listed in Section 4.1. Appropriate engineering design and analysis techniques will be used to make design selections to satisfy these requirements. Regulatory requirements must include nuclear safety considerations to protect the offsite public and onsite workers from radioactive materials. The design cannot be advanced beyond that to meet the end user requirements without establishing the safety design approach to be implemented in the design. The safety design approach includes the selection of materials and design features for the reactor components, characterization of the sources of radioactive material, selection and arrangement of radionuclide transport barriers, definition of safety functions to protect these barriers, and selection of SSCs for the performance of these and other energy production functions. The safety design approach must anticipate the challenges to the safety case that include those unique and specific to the reactor technology.

Hence, it is necessary for the designer to perform an early assessment to identify the LBEs that frame the necessary safety analyses that will be performed to demonstrate adequacy of the safety case. These analyses include those necessary and sufficient to confirm the adequacy of DID in the prevention and mitigation of accidents. Given these considerations, it is clear that the selection of LBEs must begin early in the design process in order to optimize the design in meeting end user and regulatory requirements and to avoid costly back-fits that might otherwise occur during late stages of design and licensing.

A flow chart indicating the tasks to identify and evaluate LBEs in concert with the design evolution is shown in Figure 4-8. These tasks are intended to be carried out by the design and design evaluation teams responsible for establishing the key elements of the safety case and preparing a license application. The process is used to prepare an appropriate licensing document (e.g., licensing topical report) that documents the derivation of the LBEs, which would be reviewed by the regulator as part of license review. The design and design evaluation teams are responsible for selecting the LBEs and the regulator is responsible to review and approve the selections as well as the process used for the selections. Although it is anticipated that NRC would review the entire LBE selection and evaluation process, the specific tasks with increased regulatory involvement are identified in the figure. The LBE selection and evaluation process is implemented in the following LBE selection tasks: 


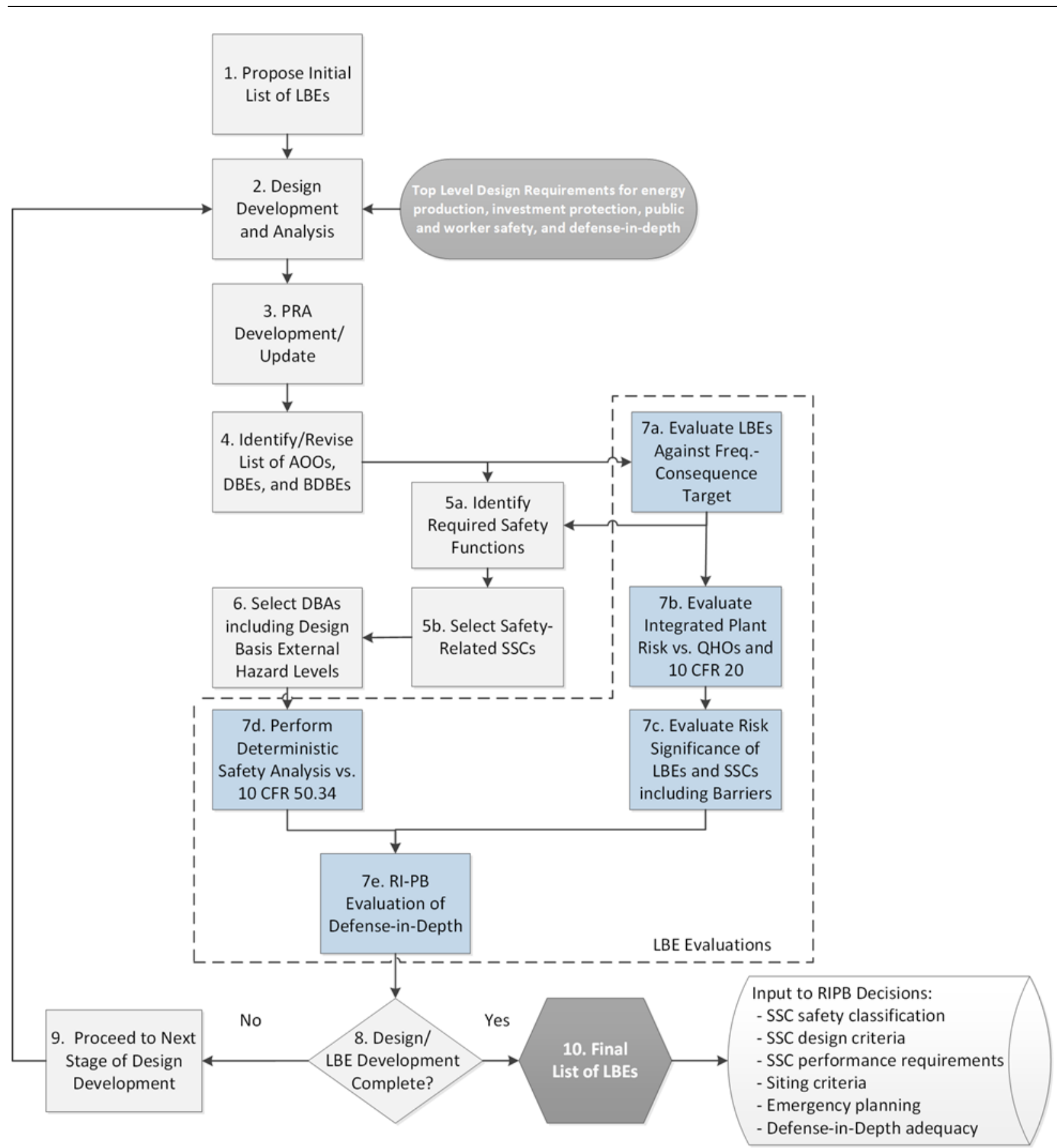

Figure 4-8. Process for Selecting and Evaluating Licensing Basis Events

The LBE selection and evaluation process is implemented in the LBE selection tasks described below.

\section{Task 1: Propose Initial List of LBEs}

During design development, it is necessary to select an initial set of LBEs which may not be complete but are necessary to develop the basic elements of the safety design. These events are to be selected deterministically and may be supported by qualitative risk insights based on all 
relevant and available experience, including prior experience from the design and licensing of reactors. The initial selection of events can also be supported by analysis techniques such as FMEAs, HAZOPs, and Master Logic Diagrams. In many cases, the designer may also have an initial assessment regarding which SSCs may be classified as SR to meet the safety design objectives for the reactor design. This classification would also be deterministically based and may be supported by qualitative risk insights using the same information utilized for the initial selection of LBEs.

\section{Task 2: Design Development and Analysis}

Design development is performed in phases and often includes a pre-conceptual, conceptual, preliminary, and final design phase and may include iterations within phases. Design development and analysis includes definition of the elements of the safety design approach, the design features to meet the top-level design requirements for energy production and investment protection, and analyses to develop sufficient understanding to perform a PRA and the deterministic safety analyses. The subsequent Tasks 3 through 10 may be repeated for each design phase or iteration until the list of LBEs becomes stable and is finalized. Because the selection of deterministic DBAs requires the selection of SR SSCs, this process also yields the selection of SR SSCs that are needed for the deterministic safety analysis in Task 7d.

\section{Task 3: PRA Development/Update}

A PRA model is developed and then updated as appropriate for each phase of the design. Prior to the first introduction of the PRA, it is necessary to develop a technically sound understanding of the potential failure modes of the reactor concept, how the plant would respond to such failure modes, and how protective strategies can be incorporated into formulating the safety design approach. The incorporation of safety analysis methods appropriate to early stages of design, such as FMEA and PHA, provide early stage evaluations that are systematic, reproducible, and as complete as the current stage of design permits. The PRA models the response of each SSC in the plant that performs a function to prevent or mitigate a release of radioactive material from any radionuclide source within the scope of the PRA. These SSC functions are defined in the LMP methodology as PSFs.

Developers are encouraged to begin developing the PRA early to support all design phases. However, developers have flexibility regarding when to introduce and develop the PRA to improve upon the initial risk management strategies or intentionally conservative analyses and related design features. If undertaken during the early design phases, the PRA is of limited scope, comprises a coarse level of detail, and makes use of engineering judgment much more than would a completed PRA that meets applicable PRA standards. The scope and level of detail of the PRA are enhanced as the design matures and siting information (or site envelope) is defined. For modular reactor designs, the event sequences modeled in the PRA should include event sequences involving single or multiple reactor modules or radionuclide sources. This approach provides useful risk insights into the design to ensure that event sequences involving multiple reactor modules are not risk-significant. The PRA process exposes sources of uncertainty encountered and provides estimates of the frequencies and doses for each LBE, including a quantification of the impacts of uncertainties using quantitative uncertainty analyses and supported by sensitivity analyses. 


\section{Task 4: Identify/Revise List of AOOs, DBEs, and BDBEs}

The event sequences modeled and evaluated in the PRA are grouped into event sequence families, each having a similar IE, challenge to the PSFs, plant response, end state, and mechanistic source term if there is a radiological release. Each of these families is assigned to an LBE category based on mean event sequence frequency of occurrence per plant-year summed over all the event sequences in the LBE family. The event sequence families from this task may confirm or revise the initial events identified in Task 1.

AOOs are off-normal events that are expected to occur in the life of the plant with frequencies exceeding $10^{-2}$ /plant-year, where a plant may be comprised of multiple reactor modules. DBEs are less frequent events that may occur in a plant with frequencies between $10^{-4}$ to $10^{-2}$ /plantyear. BDBEs are rare events with frequencies less than $10^{-4}$ /plant-year but with upper bound frequencies greater than $5 \times 10^{-7} /$ plant-year. LBEs could involve release of radioactive material and could involve two or more reactor modules or radionuclide sources. For LBEs with no radiological release, it is important to identify challenges to SSCs, including barriers that are responsible for preventing or mitigating a release of radioactive material. Such insights are important inputs to the subsequent task of identifying the RSFs. The evaluation of the consequences of LBEs is supported by mechanistic source terms.

Event sequences with upper $95^{\text {th }}$ percentile frequencies less than $5 \times 10^{-7} /$ plant-year are retained in the PRA results and used to confirm that there are no cliff-edge effects. They are also taken into account in the RIPB evaluation of DID in Task 7e.

Tasks 5 and 6 are performed together rather than sequentially. Examples are provided in this report for HTGRs, SFRs, and MSRs to illustrate how this tasks were implemented for these reactors.

\section{Task 5a: Identify Required Safety Functions}

In Task 5a, the full set of DBEs are examined to identify the PSFs that are necessary and sufficient to meet the F-C Target for all DBEs and high-consequence BDBEs, and to conservatively ensure that 10 CFR 50.34 dose requirements can be met. These PSFs are defined in the LMP methodology as RSFs). High consequence BDBEs are those with consequences that exceed 10 CFR 50.34 dose criteria. For the DBEs these PSFs, when fulfilled, are responsible for mitigating the consequences within the F-C Target. RSFs for any high-consequence BDBEs are responsible for preventing them from increasing in frequency into the DBE region and outside the F-C Target by exhibiting sufficient reliability performance to keep the BDBE frequency sufficiently low.

\section{Task $5 b$ Select/Revise Safety-Related SSCs}

For each of these RSFs identified in Task 5a, a decision is made on which set of SSCs is selected to perform these RSFs among those found to be available on each DBE. As a result of this selection, each DBEs is protected by a set of SR SSCs to perform each RSF. Structures and physical barriers that are necessary to protect any SR SSCs in performing their RSFs in response to any design basis external event are also classified as SR. SR SSCs are also selected for any $\mathrm{RSF}$ associated with any high-consequence BDBEs in which the reliability of the SSC is 
necessary to keep the event in the BDBE frequency region. The remaining SSCs that are not classified as SR are considered in other evaluation tasks including Tasks 7b, 7c, 7d, and 7e. Those SSCs not classified as SR are further subdivided to identity additional safety significant SSCs that meet risk significance and DID adequacy criteria as explained more fully in the companion report on SSC safety classification. Performance targets and design criteria for both SR and non-safety-related SSCs are developed and described more fully in the SSC safety classification report.

\section{Task 6: Select Deterministic DBAs and Design Basis External Hazard Levels}

For each DBE identified in Task 4, a deterministic DBA is defined that includes the RSF challenges represented in the DBE but assumes that the RSFs are performed exclusively by SR SSCs, and all non-safety-related SSCs that perform these same functions are assumed to be unavailable. These DBAs are then used in DBA analysis of the license application for supporting the conservative deterministic safety analysis.

RG 1.203, "Transient and Accident Analysis Methods," provides additional discussion of developing appropriate evaluation models for analyzing DBAs. The selection of conservative assumptions to be used in the DBA analysis will be informed by the quantitative uncertainty analysis of consequences that will be performed for the corresponding DBEs. In view of the fact that advanced non-LWRs will employ a diverse combination of inherent, passive, and active design features to perform the RSFs across layers of defense, and, taking into account the fact that the reactor safety design approach will be subjected to an evaluation of DID adequacy, the application of a single failure criterion is not deemed to be necessary.

A set of DBEHLs will be selected to form an important part of the design and licensing basis. This will determine the design basis seismic events and other external events that the SR SSCs will be required to withstand. When supported by available methods, data, design, site information, and supporting guides and standards, these DBEHLs will be informed by a probabilistic external hazards analysis and will be included in the PRA after the design features that are incorporated to withstand these hazards are defined. Other external hazards not supported by a probabilistic hazard analysis will be covered by DBEHLs that are determined using traditional deterministic methods.

In many cases, it is expected that the initial selection of SR SSCs and selection of the DBAs will be based on a PRA that includes internal events but has not yet been expanded to address external hazards. With the understanding that SR SSCs are required to be capable of performing their RSFs in response to external events within the DBEHL, there will be no new DBAs introduced by external hazards.

Some design basis external events such as external floods or seismic events may impact multiple reactor modules concurrently; therefore, a design objective would be to prevent a substantial release for such events.

The codes and thermal hydraulic models used within the PRA will be subject to the technical adequacy requirements in the supporting PRA standards, whereas the codes and models used in DBA analysis are expected to satisfy RG 1.203 requirements for evaluation models. 


\section{Task 7: Perform LBE Evaluations}

The deterministic and probabilistic safety evaluations that are performed for the full set of LBEs are covered in the five sub-tasks described below.

\section{Task 7a: Evaluate LBEs Against F-C Target}

In this task, the results of the PRA which have been organized into LBEs will be evaluated against an F-C Target as shown in Figure 4-5. The figure does not define specific acceptance criteria for the analysis of LBEs but rather serves as a tool to focus the attention of the designer and those reviewing the design and related operational programs to the most significant events and possible means to address those events. The NRC's Advanced Reactor Policy Statement includes expectations that advanced reactors should provide enhanced margins of safety. The safety margin between the design-specific PRA results and the F-C Target provides one useful and practical demonstration of how the design fulfills the NRC's expectations for enhanced safety. These margins also are useful in the evaluation of DID adequacy in Task $7 \mathrm{~d}$. The evaluations in this task are performed for each LBE separately. The mean values of the frequencies are used to classify the LBEs into AOOs, DBEs, and BDBE categories. However, when the uncertainty bands defined by the $5^{\text {th }}$ percentile and $95^{\text {th }}$ percentile of the frequency estimates straddles a frequency boundary, the LBE is evaluated in both LBE categories. An LBE with mean frequency above $10^{-2} /$ plant-year and $5^{\text {th }}$ percentile less than $10^{-2} /$ plant-year is evaluated as an AOO and DBE. An LBE with a mean frequency less than 104 /plant-year with a $95^{\text {th }}$ percentile above $10^{-4} /$ plant-year is evaluated as a BDBE and a DBE. An event sequence family with a mean frequency less than $5 \times 10^{-7} /$ plant year but with a $95^{\text {th }}$ percentile frequency estimate above $5 \times 10^{-7} /$ plant-year is evaluated as a BDBE. Uncertainties about the mean values are used to help evaluate the results against the frequency-consequence criteria and to identify the margins against the criteria.

DBE doses are evaluated against the F-C Target based on the mean estimates of consequence. This approach is based on the fact that the use of a conservative dose evaluation is appropriate for the deterministic safety analysis in Task 7a but is not consistent with the way in which uncertainties are addressed in risk-informed decision-making in general, where mean estimates supported by a robust uncertainty analysis are generally used to support risk significance determinations. When evaluating risk significance, comparing risks against safety goal QHOs, and evaluating changes in risk against the RG 1.174 change in risk criteria, the accepted practice has been to first perform a quantitative uncertainty analysis and then to use the mean values to compare against the various goals and criteria, which are set in the context of uncertainties in the risk assessments. These assessments apply to both the frequency and consequence estimates.

The primary purpose of comparing the frequencies and consequences of LBEs against the F-C Target is to evaluate the risk significance of individual LBEs. The objective for this activity is that uncertainties in the risk assessments are evaluated and included in discussions of design features and operational programs related to the most significant events and possible compensatory measures to address those events. The evaluations in this task are based on mean frequencies and mean doses for all three LBE categories. Three exceptions to this are that BDBEs with large uncertainties in their frequencies are evaluated as DBEs when the upper $95^{\text {th }}$ percentile of the frequency exceeds $10^{-4}$ /plant-year and as BDBEs when the upper $95^{\text {th }}$ percentile 
frequencies are greater than $5 \times 10^{-7} /$ plant-year even when the mean frequency is less than $5 \times 10^{-7} /$ plant year. AOOs with lower $5^{\text {th }}$ percentile frequencies below $10^{-4} /$ plant-year are also evaluated as DBEs. The uncertainties about these means are considered as part of the RIPB DID evaluation in Task 7e.

The PRA process exposes sources of uncertainty encountered in the assessment of risk and provides estimates of the frequencies and doses for each LBE, including a quantification of the impacts of uncertainties using quantitative uncertainty analyses and supporting sensitivity analyses. Sources of uncertainty that are identified by the PRA and not fully resolved via quantification are addressed as part of a risk-informed evaluation of DID, as discussed in the LMP report on DID. The evaluation of the consequences of all LBEs are supported by mechanistic source terms and a quantitative uncertainty analysis.

The upper bound consequences for each DBA, defined as the $95^{\text {th }}$ percentile of the uncertainty distribution, shall meet the 10 CFR 50.34 dose limit at the EAB. Sources of uncertainty in both frequencies and consequences of LBEs are identified and addressed in the LMP approach to DID.

A function of the LBE frequency-dose evaluation is to ensure that LBEs involving radiological releases from two or more reactor modules do not make a significant contribution to risk and to ensure that measures are taken to manage the risks of multi-reactor module or multi-source events.

The final element of the LBE evaluation in this task is to identify design features that are responsible for keeping the LBEs within the F-C Target including those design features that are responsible for preventing or mitigating risk-significant releases for those LBEs with this potential. This evaluation leads to performance targets and design criteria that are developed within the process of the SSC classification task in the risk-informed, performance-based approach. More discussion of this point is found in the LMP report on SSC safety classification.

\section{Task 7b. Evaluate Integrated Plant Risk Against QHOs and 10 CFR 20}

In this task, the integrated risk of all the LBEs, is evaluated against three cumulative risk targets:

- The total mean frequency of exceeding a site boundary dose of 100 mrem from all LBEs should not exceed 1/plant-year. This metric is introduced to ensure that the consequences from the entire range of LBEs from higher frequency, lower consequences to lower frequency, higher consequences are considered. The value of $100 \mathrm{mrem}$ is selected from the annual cumulative exposure limits in 10 CFR 20.

- The average individual risk of early fatality within 1 mile of the EAB from all LBEs based on mean estimates of frequencies and consequences shall not exceed $5 \times 10^{-7} /$ plant-year to ensure that the NRC Safety Goal QHO for early fatality risk is met.

- The average individual risk of latent cancer fatalities within 10 miles of the EAB from all LBEs based on mean estimates of frequencies and consequences shall not exceed $2 \times 10^{-6} /$ plant-year to ensure that the NRC Safety Goal QHO for latent cancer fatality risk is met. 
One element of this task is to identify design features that are responsible for preventing and mitigating radiological releases and for meeting the integrated risk criteria. This evaluation leads to performance targets and design criteria that are developed within the process of the SSC classification task as discussed in the LMP report on SSC safety classification.

In addition to the two QHOs, the 10 CFR 20 criterion is considered in recognition that the referenced regulatory requirement is for the combined exposures from all releases even though it has been used in developing the F-C Target used for evaluating the risks from individual LBEs. Having these cumulative risk targets as part of the process provides a mechanism to ensure that the F-C Target is conservatively defined for use as a tool for focusing attention on matters important to managing the risks from non-LWRs.

\section{Task 7c. Evaluate Risk Significance of LBEs and SSCs Including Barriers}

In this task, the details of the definition and quantification of each of the LBEs in Task $7 \mathrm{a}$ and the integrated risk evaluations of Task $7 \mathrm{~b}$ are used to define both the absolute and relative risk significance of individual LBEs and SSCs which include radionuclide barriers. These evaluations include the use of PRA risk importance metrics, where applicable, and the examination of the effectiveness of each of the layers of defense in retaining radionuclides. LBEs are classified as risk-significant if the LBE site boundary dose exceeds 2.5 mrem over 30 days and the frequency of the dose is within 1\% of the F-C Target. SSCs are classified as risk-significant if the SSC function is necessary to keep any LBEs inside the F-C Target, or if the total frequency of LBEs with the SSCs failed is within 1\% of any of the three cumulative risk targets identified in Task $7 \mathrm{~b}$. This information is used to provide risk insights, to identify safetysignificant SSCs, and to support the RIPB evaluation of DID in Task 7e.

\section{Task 7d. Perform Deterministic Safety Analyses Against 10 CFR 50.34}

This task corresponds to the traditional deterministic safety analysis that is found in the DBA analysis of the license application. It is performed using conservative assumptions. The uncertainty analyses in the mechanistic source terms and radiological doses that are part of the PRA are available to inform the conservative assumptions used in this analysis and to avoid the arbitrary "stacking" of conservative assumptions.

\section{Task 7e. Risk-Informed, Performance-Based Evaluation of DID}

In this task, the definition and evaluation of LBEs should be used to support a RIPB evaluation of DID. This task involves the identification of risk-significant sources of uncertainty in both the frequency and consequence estimates, and evaluation against DID criteria. Outcomes of this task include possible changes to the design to enhance the plant capabilities for DID, formulation of conservative assumptions for the deterministic safety analysis, and input to defining and enhancing programmatic elements of DID.

It is noted that this DID evaluation does not change the selection of LBEs directly. This evaluation could lead to compensatory actions that change the design capability or programmatic controls on the design, which in turn would lead to changes in the definition of LBEs and in the estimation of their frequencies and consequences and thereby affect the evaluation of LBEs. 
This may be a task where designers to assess plant features for effective satisfaction of regulatory requirements such as 10 CFR 50.155, "Mitigation of Beyond-Design Basis Events," and 10 CFR 73, "Physical Protection of Plants and Materials." The results from the evaluation can also support related licensing matters such as defining appropriate constraints in terms of siting (i.e., 10 CFR 100, "Reactor Site Criteria"), offsite emergency planning, and development of plant procedures and guidelines.

The process and criteria to establish the adequacy of DID are discussed in the companion LMP report on DID.

\section{Task 8 Decide on Completion of Design/LBE Development}

The purpose of this task is to decide if additional design development is needed, either to proceed to the next logical stage of design or to incorporate feedback from the LBE evaluation that design, operational, or programmatic improvements should be considered to meet the risk targets. Such design improvements could be motivated by a desire to increase margins against the frequency-consequence criteria, reduce uncertainties in the LBE frequencies or consequences, manage the risks of multi-reactor module events, limit the need for restrictions on siting or emergency planning, or enhance the performance against DID criteria. The DID adequacy evaluation may result in the need for additional iterations on the adequacy of design, operational, and programmatic programs, which in turn could influence the PRA and result in a need for cycling through some or all the LBE evaluation tasks.

\section{Task 9 Proceed to Next Stage of Design Development}

The decision to proceed to the next stage of design is reflected in this task. This implies not only completion of the design but also confirmation that DID criteria evaluated in Task 7e have been satisfied. Open items or unverified assumptions should be resolved at later stages of design development.

\section{Task 10. Finalize List of LBEs and Safety-Related SSCS}

Establishing the final list of LBEs and SR SSCs signifies the completion of the LBE selection process and the selection of the SR SSCs. The next task in implementing the TI-RIPB approach is to complete the SSC safety classification process and to formulate performance targets and design criteria for SSCs that are necessary to control the LBE frequencies and doses and other performance and special treatment requirements associated with the protection of fission product barriers. Important information from Task $7 \mathrm{a}$ through $7 \mathrm{e}$ is used for this purpose.

The LMP tasks illustrated in Figure 4-8 are focused on the selection and evaluation of LBEs. Because the definition of DBAs involves the need to determine the RSFs and the selection of SR SSCs to perform these RSFs, this chart addresses part of the LMP process for SSC safety classification. In addition, the plant capabilities for DID are addressed in Task 7e. As explained more fully in the companion report on SSC safety classification, there are additional tasks needed to complete the LMP tasks for SSC safety classification. In addition, Task 7e is just part of the DID evaluation as discussed in the LMP report on DID. A more holistic view of all the tasks in the LMP methodology is contained in the industry guidance document NEI 18-04 and in the entire collection of LMP reports. 


\subsubsection{Evolution of LBEs Through Design and Licensing Stages}

The LBE selection flow chart in Figure 4-8 reflects an iterative process involving design development, PRA development, selection of LBEs, and evaluation of LBEs. The process flow chart can be viewed as beginning in the pre-conceptual or conceptual design phase when many design details are unavailable, the PRA effort has not begun, and the safety design approach is just being formulated. To begin the process outlined in Figure 4-8, an initial set of LBEs is proposed based on engineering judgment in Task 1 of the process. This may generate an initial target selection of SR SSCs.

During the conceptual design phase, different design concepts are explored, and alternatives are considered to arrive at a feasible set of alternatives for the plant design. The effort to develop a PRA should begin during this phase. Traditional design and analysis techniques are applied during conceptual design, including (1) use of traditional design bases of engineering analysis and judgment, (2) application of research and development programs, (3) use of past design and operational experience, (4) performance of design trade studies, and (5) decisions on how or whether to conform to established applicable LWR-based reactor design criteria and whether other principle criteria are needed.

Creation of the initial event list of LBEs includes expert evaluation and review of the relevant experience gained from previous reactor designs and associated PRAs, when available. It starts by answering the first question in the risk triplet: "What can go wrong?" Care must be exercised to ensure that information taken from other reactor technologies is interpreted correctly for the reactor technology in question. The body of relevant reactor design and PRA data that is available to draw upon may vary for different reactor technologies. Once design alternatives and trade studies are developed, the safety design approach can be defined. A review of the major systems can take place, and techniques such as a FMEAs and PHAs such as HAZOPs can be applied to identify initial failure scenarios and to support the initial PRA tasks to define IEs.

Preliminary design activities need to balance regulatory and design requirements, cost, schedule, and other user requirements to optimize the design, cost, and capabilities that satisfy the objectives for the reactor facility.

As the design matures, the scope and level of detail of the PRA is expanded and is used to help support design decisions along the way. An early simplified PRA can be very helpful to support design trade studies that may be performed to better define the safety design approach. Questions that arise in the efforts to build a PRA model may be helpful to the design team especially in the mutual understanding of what kind of challenges will need to be addressed. Because the design is being changed more frequently at this point and better characterized as the design phases evolve, the PRA results and their inputs to the LBE selection process will also be subject to change.

As a result, refinements to the list of LBEs are expected. The simplifying perception that a design has stages that contain bright lines is a frequent description at the system level but is not correct at the plant level. Different parts of the design mature at different times. Systems often go through design stages like this, however, at any moment, there may be systems in many 
design phases simultaneously. Consequently, the PRA development is a continuum as well, maturing with the systems design. PRA updates with system development then provide a more frequent, integrated plant performance check that is otherwise missing in the conventional design process and will also provide risk insights to help the design decisions. When the design, construction, and PRA are developed in a manner that is sufficient to meet PRA requirements reflected in applicable PRA standards and regulatory guides, the LBEs can be finalized and included in the license application.

\subsubsection{Role of the PRA in LBE Selection}

The primary motivation to utilize inputs from a PRA in the selection of LBEs is that it is the only method available that has the capability to identify the events and the associated risks that are specific and unique to a new reactor design. Traditional methods for selecting LBEs, such as those reflected in the General Design Criteria and Chapter 15 of the Standard Review Plan, do not refer to a systematic method for identifying design specific events. The generic lists of events provided in the SRP guidance as examples for transients and postulated accidents to consider are specific to LWRs. Traditional systems analysis techniques that can be used to evaluate a design and were used to define the LBEs for currently licensed reactors, including FMEAs, HAZOPs, single failure analyses, etc., have been incorporated into PRA methodology for selecting IEs and developing event sequence models.

PRA is also a mature technology that is supported by industry consensus standards and regulatory guides. ${ }^{[35][36][41]}$ There are no similar consensus standards for deterministic selection of LBEs for new non-LWR reactor designs. Although much of the available experience in PRA has been with operating LWR plants, there is a rich history of PRA as applied to advanced nonLWR designs including HTGRs, MAGNOX and AGRs, and liquid-metal-cooled fast reactors. A trial use PRA standard for advanced non-LWRs was issued by the ASME/ANS Joint Committee on Nuclear Risk Management in 2013 and, by 2019, a revised version for consideration as an ANSI standard is scheduled to be available for ballot. The trial use PRA standard has been subjected to a number of PRA pilot studies on the PRISM, HTR-PM, and several other nonLWR designs. Lessons from these pilot studies are being incorporated into the revised nonLWR PRA standard.

Prior to first introduction of the PRA, it is necessary to develop a technically sound understanding of the potential failure modes of the reactor concept, how the reactor plant would respond to such failure modes, and how protective strategies will be incorporated into formulating the safety design approach. The incorporation of safety analysis methods appropriate to early stages of design, such as FMEA and PHA, provide industry-standardized practices to ensure that such early stage evaluations are systematic, reproducible, and as complete as the current stage of design permits.

The interfaces between traditional systems engineering processes and the initial development of the PRA model are shown in Figure 4-9. It is important to note that the systems engineering inputs on the left-hand side of the diagram are fundamental to developing the design. However, with the concurrent development of the PRA model, the PRA is developed in parallel with the design and thereby is available to provide important risk insights to the design development and 
supporting systems analyses. Decisions to defer the introduction of the PRA to later stages of design lead to reduced opportunities for cost-effective risk management.

Systems Engineering Inputs

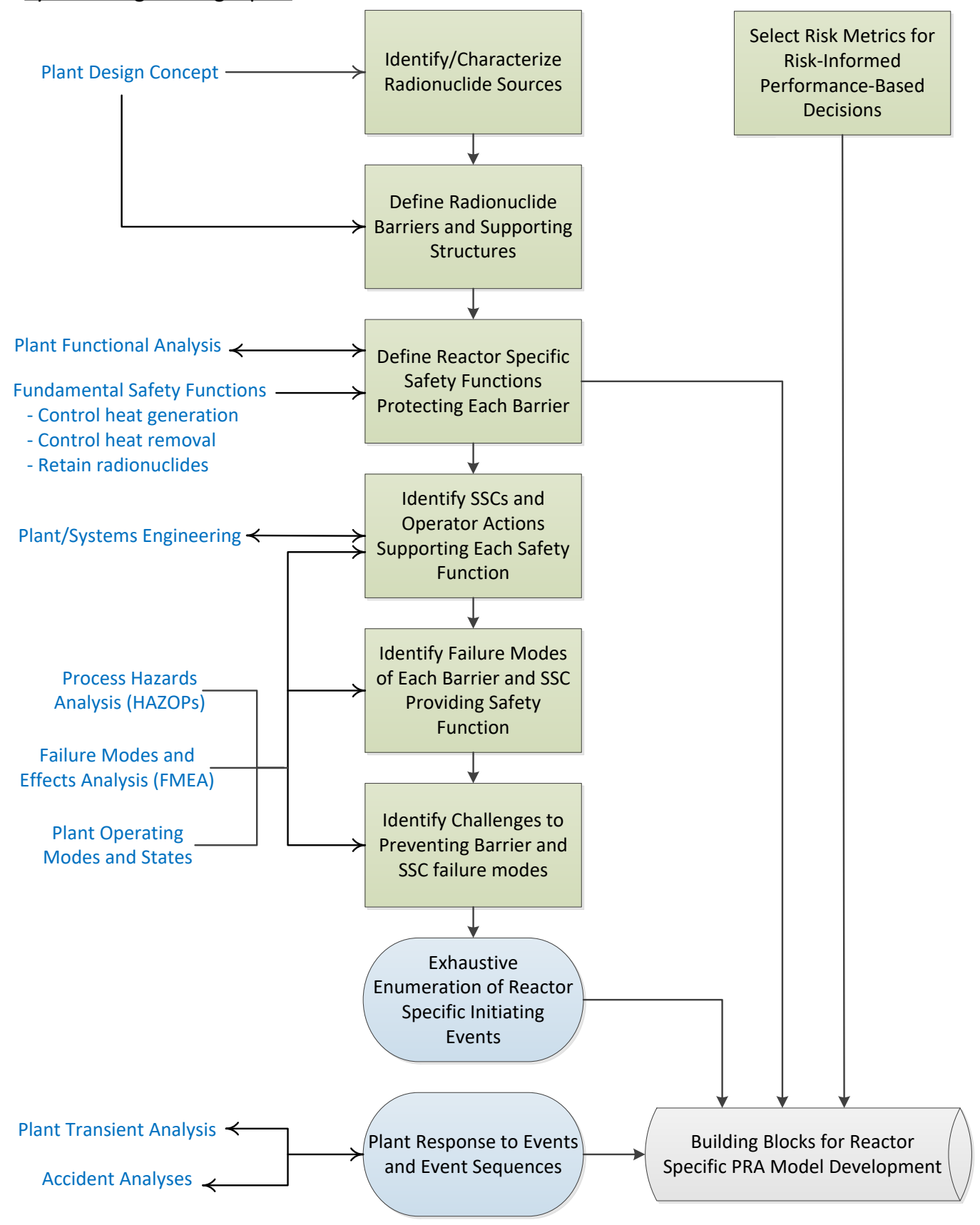

Figure 4-9. Flow Chart for Initial PRA Model Development

The PRA will be used to evaluate the safety characteristics of the design and to provide a structured methodology from which the initial set of LBEs will be risk-informed. The evaluation of the risks of the LBEs against the F-C Target help makes the LBE selection process both risk- 
informed and performance-based. This evaluation methodology is critical to the development of a revised licensing framework. It highlights the issues that deserve the greatest attention in a safety-focused process. Subsequently the PRA will provide important input to the formulation of performance targets for the capability and reliability of the SSCs to prevent and mitigate accidents and thereby contribute to the performance-based aspects of the design and licensing development process. In addition, engineering judgment and utilization of relevant experience will continue to be used to ensure that LBE selection and classification is complete.

The PRA will systematically enumerate event sequences and assess the frequency and consequence of each event sequence. Event sequences will include internal events, internal plant hazards, and external events. The modeled event sequences will include the contributions from common cause failures and will not arbitrarily exclude sequences that exceed the single failure criterion.

Each event sequence family reflected in the LBE definitions is defined as a collection of event sequences that similarly challenge plant safety functions. This means that the IEs within the family have a similar impact on the plant such that the event sequence development following the plant response will be the same for each sequence within the family. If the event sequence involves a release, each sequence in the family will have the same mechanistic source term and offsite radiological consequences. Many of the LBEs do not involve a release. Understanding the plant capabilities to prevent release is an extremely important insight back to the design. Event sequence family grouping facilitates selection of LBEs from many individual events into a manageable number.

The PRA's quantification of both frequencies and consequences will address uncertainties, especially those associated with the potential occurrence of rare events. The quantification of frequencies and consequences of event sequences, and the associated quantification of uncertainties, provides an objective means of comparing the likelihood and consequence of different scenarios against the F-C correlation. The scope of the PRA, when completed will be as comprehensive and sufficiently complete as a full-scope, all modes, Level 3 PRA covering a full set of internal and external events when the design is completed and site characteristics well defined. Designers may propose to address all or parts of the process by assessing fission product barriers and showing that radioactive materials are with a high degree of confidence retained within the facility. Such an approach would still require that some of the information provided by a PRA, including the identification challenges to the barriers and identification and evaluation of dependencies among the barriers, including challenges that may impact multiple reactor modules would be considered in the formulation of licensing basis events and the determination of safety significant SSCs.

The technical adequacy of the LBE selection process is expected to be enhanced considerably by risk-informing the process. In addition, the PRA will include event sequences involving two or more reactor modules, if applicable, as well as two or more source of radioactive material. This will enable the identification and evaluation of risk management strategies to ensure that sequences involving multiple modules and sources are not risk significant. Because the PRA includes a quantification of offsite radiological consequences, the risk significance of event 
sequences and SSCs will be made in consideration of the total integrated risk of the multimodule plant.

It is recognized that PRA technology has limitations, especially with regard to application to advanced non-LWRs in the design stage. The proposed LBE selection process is not risk-based, but rather risk-informed as there are strong deterministic inputs to the process. First the PRA development is anchored to traditional deterministic system engineering analyses that involve numerous applications of engineering judgment. Identified in the left side of Figure 4-9, these include FMEAs, process hazards assessment, application of relevant experience from design and licensing of other reactors, and deterministic models of the plant response to events and accidents. Second, the deterministic DBAs are selected based on prescriptive rules and analyzed using conservative assumptions. Finally, the LBE selection includes a review to ensure that the LBE selection and the results of the LBE evaluations meet a set of criteria to ensure the adequacy of DID.

These evaluations often lead to changes to the plant design and programmatic controls that are reflected in changes to the PRA and, hence, changes to the selection of LBEs and SSC safety classification. In addition to these elements, peer reviews and regulatory reviews of the PRA will provide an opportunity to challenge the completeness and treatment of uncertainties in the PRA to ensure that the deterministic DBAs and the conservative assumptions that are used in Chapter 15 are sufficient to meet the applicable regulatory requirements. A companion report on LMP approach to DID and how this approach is integrated into RIPB decision-making provides more detail on how deterministic judgments are used to support design and licensing decisions.

\subsubsection{Structuring PRA Event Sequence Logic for LBE Selection}

In principle, the structure of the PRA event tree logic should not impact the identification of Required Safety Functions (RSFs) and the selection of the Safety Related (SR) SSCs. First, RSFs are functions and not SSCs. RSFs are the reactor specific safety functions that enable the plant to fulfill the fundamental safety functions of retaining the radionuclides, controlling heat generation, and controlling heat removal. Reactors fulfill these functions with a combination of inherent reactor features, passive safety functions provided in part based on physical laws, and passive and active SSCs. The event tree top events in a PRA model capture those design features that have been assigned a failure probability model, but important aspects of the RSFs are reflected in the deterministic plant response models and mechanistic source term models that are used to demonstrate the success criteria and to resolve the conditions necessary to determine the event sequence consequences. SSCs modeled in PRA logic may be turned off and on in a binary manner to simulate their performance of the RSFs and to evaluate the SSC risk significance; it is not feasible to turn off and on in a binary manner the inherent features and some of the passive features that deliver the performance of the RSFs. The reason is even when SSCs modeled in a binary way are postulated to fail, the resulting consequences modeled in the PRA do not fully simulate the failure to perform the RSFs.

Task 5a in Figure 4-8 briefly describes the definition of RSFs as part of the LMP LBE evaluation methodology. Examples of RSFs are developed in Section 5.1 and 5.2 for MHTGR and PRISM, respectively. Each of the LMP demonstration project reports on Xe-100, ${ }^{[77]} \mathrm{PRISM},{ }^{[78]}$ 
MSRE, ${ }^{[79]}$ Kairos-FHR, ${ }^{[80]}$ and Westinghouse eVinci Micro-Reactor ${ }^{[81]}$ include examples of the RSFs for each reactor, and these are summarized in Table 4-1. Note that the RSFs are associated with specific sources of radionuclides .

Table 4-1. Examples of Required Safety Functions Identified in LMP Demonstrations

\begin{tabular}{|c|c|c|c|c|c|c|}
\hline & MHTGR* & Xe-100 & PRISM & Kairos-FHR & MSRE & $\begin{array}{c}\text { Westinghouse } \\
\text { eVinci }\end{array}$ \\
\hline $\begin{array}{l}\text { Radionuclide } \\
\text { Sources } \\
\text { Considered }\end{array}$ & $\begin{array}{l}\text { Core and } \\
\text { Reactor } \\
\text { Coolant } \\
\text { System }\end{array}$ & $\begin{array}{l}\text { Core and } \\
\text { Reactor } \\
\text { Coolant } \\
\text { System }\end{array}$ & $\begin{array}{l}\text { Reactor Core } \\
\text { only }\end{array}$ & $\begin{array}{l}\text { Core and } \\
\text { Reactor Vessel }\end{array}$ & $\begin{array}{l}\text { Fuel Salt } \\
\text { System and } \\
\text { Drain Tank }\end{array}$ & $\begin{array}{l}\text { Entire Micro } \\
\text { Reactor Plant }\end{array}$ \\
\hline $\begin{array}{l}\text { Required } \\
\text { Safety } \\
\text { Functions }\end{array}$ & $\begin{array}{l}\text { Retain Rn in } \\
\text { Fuel Particles } \\
\text { Control } \\
\text { Chemical } \\
\text { Attack } \\
\text { Control Heat } \\
\text { Generation } \\
\text { Control Heat } \\
\text { Removal }\end{array}$ & $\begin{array}{l}\text { Retain Rn in } \\
\text { Fuel Particles } \\
\text { Control } \\
\text { Chemical } \\
\text { Attack } \\
\text { Control Heat } \\
\text { Generation } \\
\text { Control Heat } \\
\text { Removal }\end{array}$ & $\begin{array}{l}\text { Remove Core } \\
\text { Heat } \\
\text { Reactivity } \\
\text { Control }\end{array}$ & $\begin{array}{l}\text { Maintain Fuel } \\
\text { Particle } \\
\text { Integrity } \\
\text { Control Core } \\
\text { Reactivity } \\
\text { Remove Decay } \\
\text { Heat } \\
\text { Maintain } \\
\text { Vessel } \\
\text { Integrity }\end{array}$ & $\begin{array}{l}\text { Maintain } \\
\text { Confinement } \\
\text { of Rns } \\
\text { Control } \\
\text { Chemical } \\
\text { Behavior } \\
\text { Control } \\
\text { Nuclear Heat } \\
\text { Generation } \\
\text { Control Heat } \\
\text { Removal and } \\
\text { Addition }\end{array}$ & $\begin{array}{l}\text { Containment } \\
\text { of } \\
\text { Radioactive } \\
\text { Material } \\
\text { Reactivity } \\
\text { Control } \\
\text { Decay Heat } \\
\text { Removal } \\
\text { Control }\end{array}$ \\
\hline
\end{tabular}

One of the RSFs for the MHTGR was identified as "control core heat removal." In considering how Design Basis Events might increase in consequences if that RSF was not fulfilled, it was determined that fuel temperatures could increase and depending on the extent to which heat removal was not controlled, the fuel temperatures could exceed ANY temperature limit including the limits where the TRISO fuel particles would fail. It was recognized that this is not a binary situation as there is no limit to how much out of control of heat removal to postulate. In reality, the core temperatures in the MHTGR DBEs (and all LBEs) are controlled using passive and inherent design features including core power density, core power limit, and passive heat removal phenomena including conduction, convection, and radiation from the core to the reactor cavity. Qualitative evaluation of the safety characteristics of the reactor where sufficient to determine that this function is an RSF for this reactor.

In other LMP applications, PRA model sensitivity analyses involving turning off and on binary elements were used to help inform the selection of RSFs. While this approach is acceptable it must be noted that such analyses are limited to seeing what the binary element is actually simulating and it may not fully capture the entire safety function.

If a PRA model is originally developed with the sole purpose of determining the level of risk without necessary supporting the LMP process, model simplifications that are made to the model

\footnotetext{
${ }^{*}$ The MHTGR example was conducted under a precursor of the LMP. ${ }^{[44]}$
} 
intended for this sole purpose might make it difficult to determine the risk significance of specific SSCs without revising the model. Hence if one were to develop the PRA model from the beginning with the purpose of supporting the LMP methodology, the construction of the model should consider the type of questions that the LMP is attempting to address such as determining the risk significance of specific SSCs.

Additional details are covered in the LMP PRA approach report. ${ }^{[5]}$ 


\subsection{EXAMPLE LBE DEVELOPMENT FOR ADVANCED NON-LWRS}

The purpose of this section is to provide examples of LBE development for different advanced non-LWR reactor technologies. In Section 5.1, LBEs that were developed for the MHTGR are presented. Examples of LBEs for the PRISM SFR are illustrated in Section 5.2. Section 5.3 summarizes initial work toward LBE development for reactors that employed molten salt coolant including the fluoride-salt-cooled, high-temperature reactor (FHR) and the Molten Salt Reactor Experiment (MSRE). Additional examples of LBEs are being defined in a series of LMP table top exercises as discussed in Section 5.4.

\subsection{Example Selection of LBEs for HTGRs}

In this section, some examples from the MHTGR Preliminary Safety Information Document ${ }^{[44]}$ and the supporting PRA ${ }^{[45]}$ are used to illustrate some of the key tasks in the LBE definition process of Figure 4-8. The basic tasks in LBE definition in this figure were first developed in the MHTGR case. The MHTGR examples presented in this section include several simplified event trees with LBE assignments; examples of AOOs, DBEs, and BDBEs; the process of safety classification of SSCs; and the selection of DBAs. Comparison of the results against the frequency-dose criteria is also provided. The example LBEs presented in this section for the MHTGR were developed to support a pre-licensing review. The derivation of the LBEs using input from the supporting PRA was documented in a licensing topical report, ${ }^{[71]}$ which was reviewed by the staff as documented in NUREG-1338.

\subsubsection{Example Event Tree Development}

The MHTGR PRA included a systematic search for IEs and included the development and quantification of the frequencies and consequences for the following categories of IEs:

- Range of helium pressure boundary (HPB) failures from small leaks to offset rupture of relief valve standpipe

- Transients with loss of main loop cooling

- Seismic events

- Loss of offsite power with turbine trip

- Anticipated transients requiring scram

- Inadvertent control rod withdrawal

- Small and large steam generator leaks

Event sequence models were developed based on plant-wide thermo-fluid" plant response analyses. For event sequences involving a release of radioactive material, mechanistic and event sequence-specific source terms and offsite radiological doses were estimated. A full quantification of uncertainties was provided to support the frequency and consequence estimates.

"Because helium is a compressible gas, the term "thermos-fluid" is used in lieu of "thermal hydraulic." 
The simplified event tree for very small leaks $\left(<0.05 \mathrm{in}^{2}\right)$ in the MHTGR HPB is shown in Figure 5-1. The frequencies and probabilities shown in the figure were derived from more detailed event trees in the MHTGR PRA. ${ }^{[45]}$ The MHTGR design is comprised of four reactor modules, and this IE impacts a single module with an estimated frequency of 0.22 /plant-year.

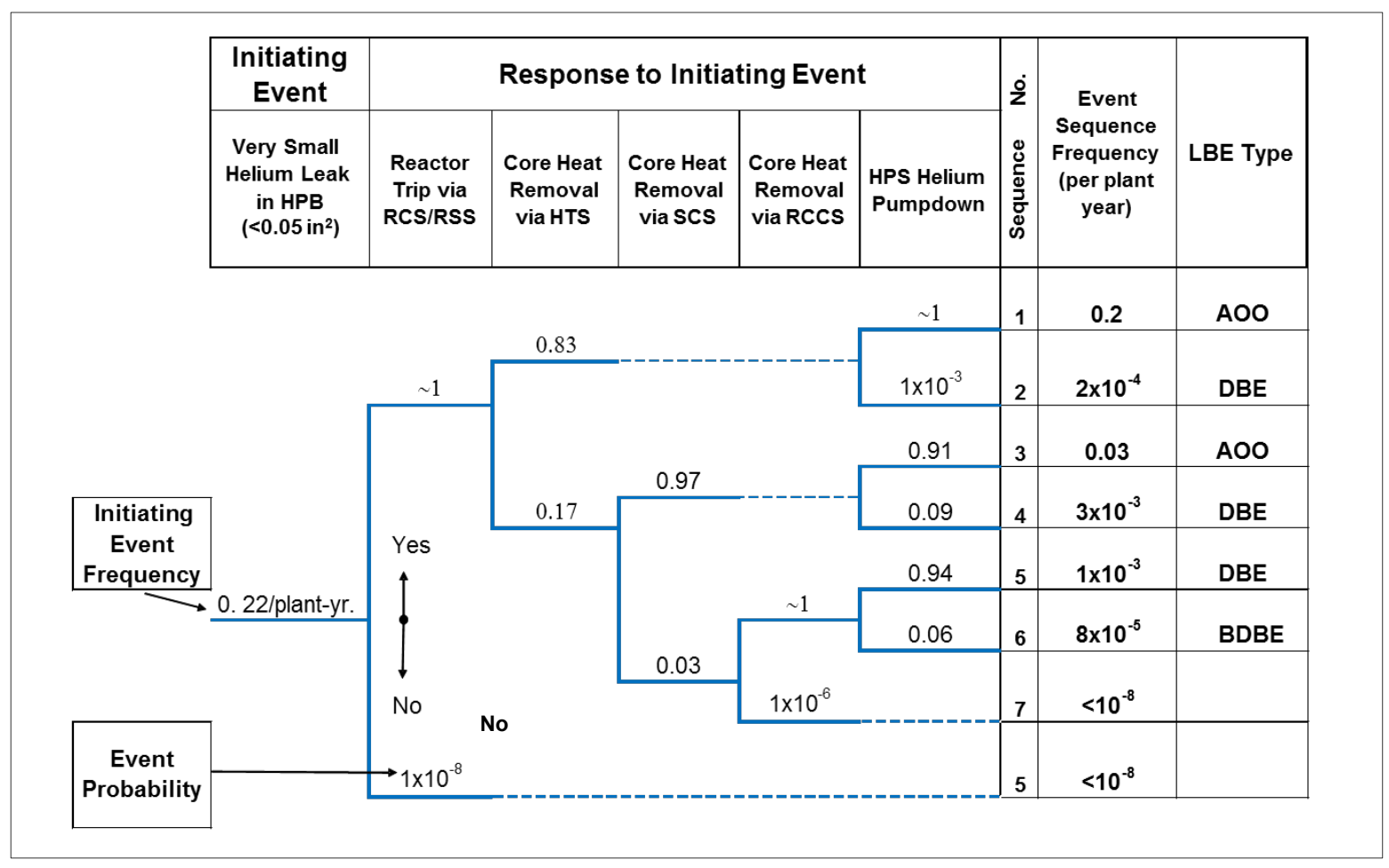

Figure 5-1. Event Tree for MHTGR Very Small Leaks in Helium Pressure Boundary

Based on high radiation levels in the reactor building with diverse signals from a reduction in primary system pressure and provisions for manual trip if automatic trip is unsuccessful, there are signals to trip the reactor via the Reactivity Control System (RCS), which is in turn backed up by the diverse Reserve Shutdown System (RSS), yielding a very low probability of failure to insert negative reactivity. Core heat removal is normally provided by continued operation of the main Heat Transport System (HTS). If that method of forced circulation cooling is unavailable, forced circulation cooling is provided by a diverse Shutdown Cooling System (SCS). If both of these systems are unavailable or fail, core heat removal is provided by a passive Reactor Cavity Cooling System (RCCS). Because the leakage of helium is very slow for this IE, there is sufficient time for the operators to use the Helium Purification and Services System to pumpdown the primary system to reduce the leakage from the system and to reduce the pressure drop across the break and the driving force for fluid release to the reactor building.

Based on the frequency of occurrence, there are two AOOs involving successful forced cooling and pump-down, two DBEs involving successful forced cooling and failure to affect the pumpdown: one DBE with loss of forced cooling with successful passive cooling via the RCCS with successful pump-down, and one BDBE with loss of forced cooling with successful RCCS cooling and no pump-down. Event sequences with frequencies below $5 \times 10^{-7} /$ plant-year are not 
classified as BDBEs, but their results are retained in the PRA documentation and evaluated to ensure there are no cliff-edge effects. All these LBEs involve a full or partial release of circulating primary coolant radioactivity, and those involving loss of forced cooling are also subject to a small delayed fuel release into the reactor building.

Figure 5-2 provides an example event tree for an event that challenges all four reactor modules. The IE for this case is a loss of offsite power and trip of all four turbine generators, each of which is designed to remain online to supply the house load for AC power.

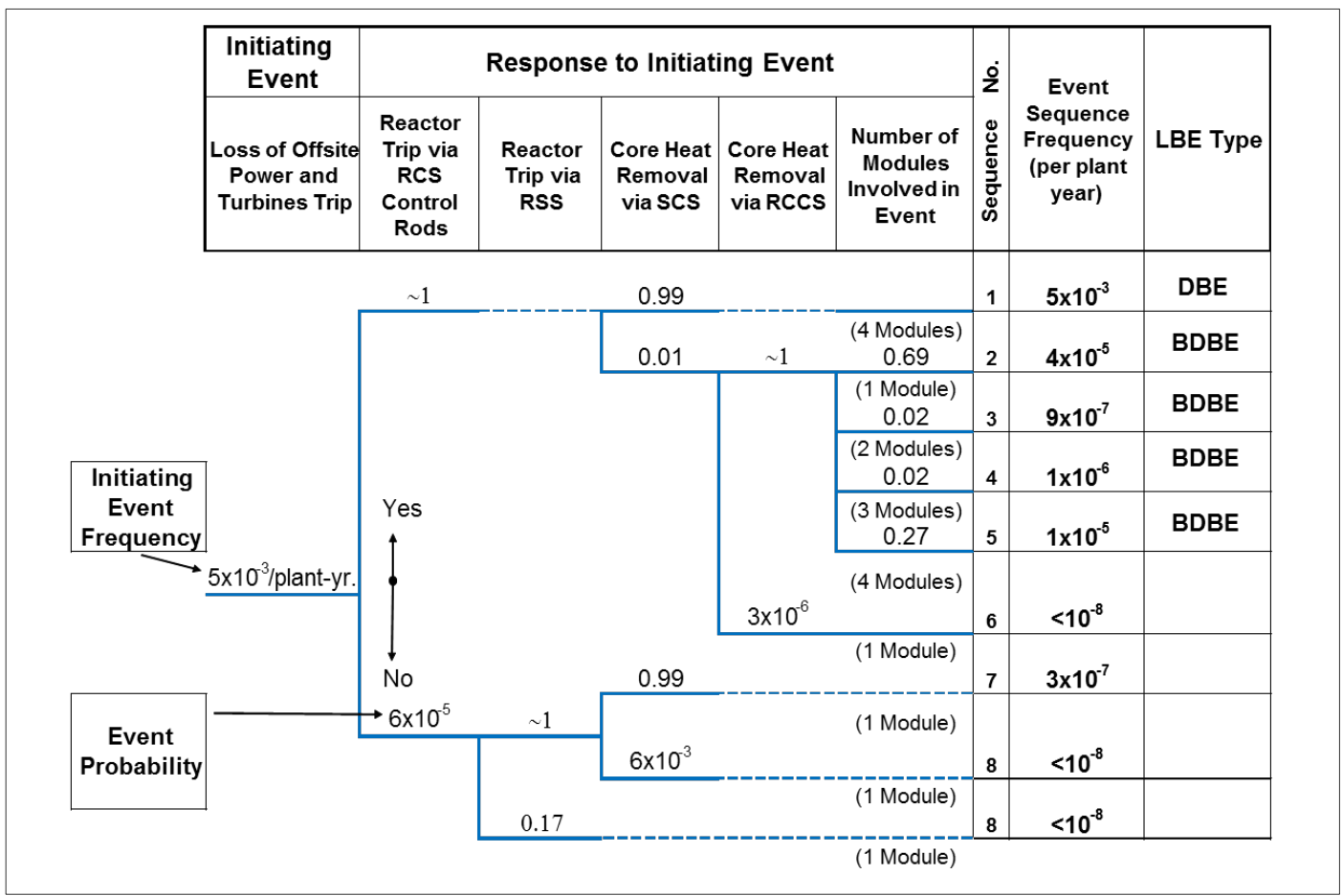

Figure 5-2. Event Tree for MHTGR Loss of Offsite Power and Turbine Trip

As with the previous example, the event tree includes the expected responses of the RCS and RSS to trip the reactor. Because the IE takes out the main HTS possibility for forced cooling, the only option for forced cooling in this case is the SCS. There is one DBE for the case where there is successful forced cooling on all four reactor modules and four BDBEs in which there is a loss of forced cooling on one, two, three, or all four modules.

A third example event tree from the MHTGR PRA is shown in Figure 5-3. The IE is an offset rupture of a steam generator tube. The reactor protection systems are designed to detect moisture in the primary system whose signals are backed up by high primary system pressure caused by the moisture ingress to the primary coolant. Additional protection to limit moisture ingress is provided by isolating the secondary side of the steam generator (SG) and then dumping the remaining water and steam into dump tanks inside the reactor building. With successful isolation and continued forced cooling via SCS there is insufficient primary pressure increase to lift the helium pressure relief valves. For failure to isolate or failure of continued forced cooling there is sufficient pressure increase to lift the helium relief valves, which when challenged may 
open and reclose or may open and fail to close. For sequences in which there is no continued forced cooling and lifting of the helium valves the delayed fuel releases are enhanced somewhat due to the chemical attack on the fuel. This event tree produces one AOO with successful plant response of all functions, one DBE with loss of forced cooling after successful isolation and dump, and three BDBEs only one of which involves a loss of forced cooling.

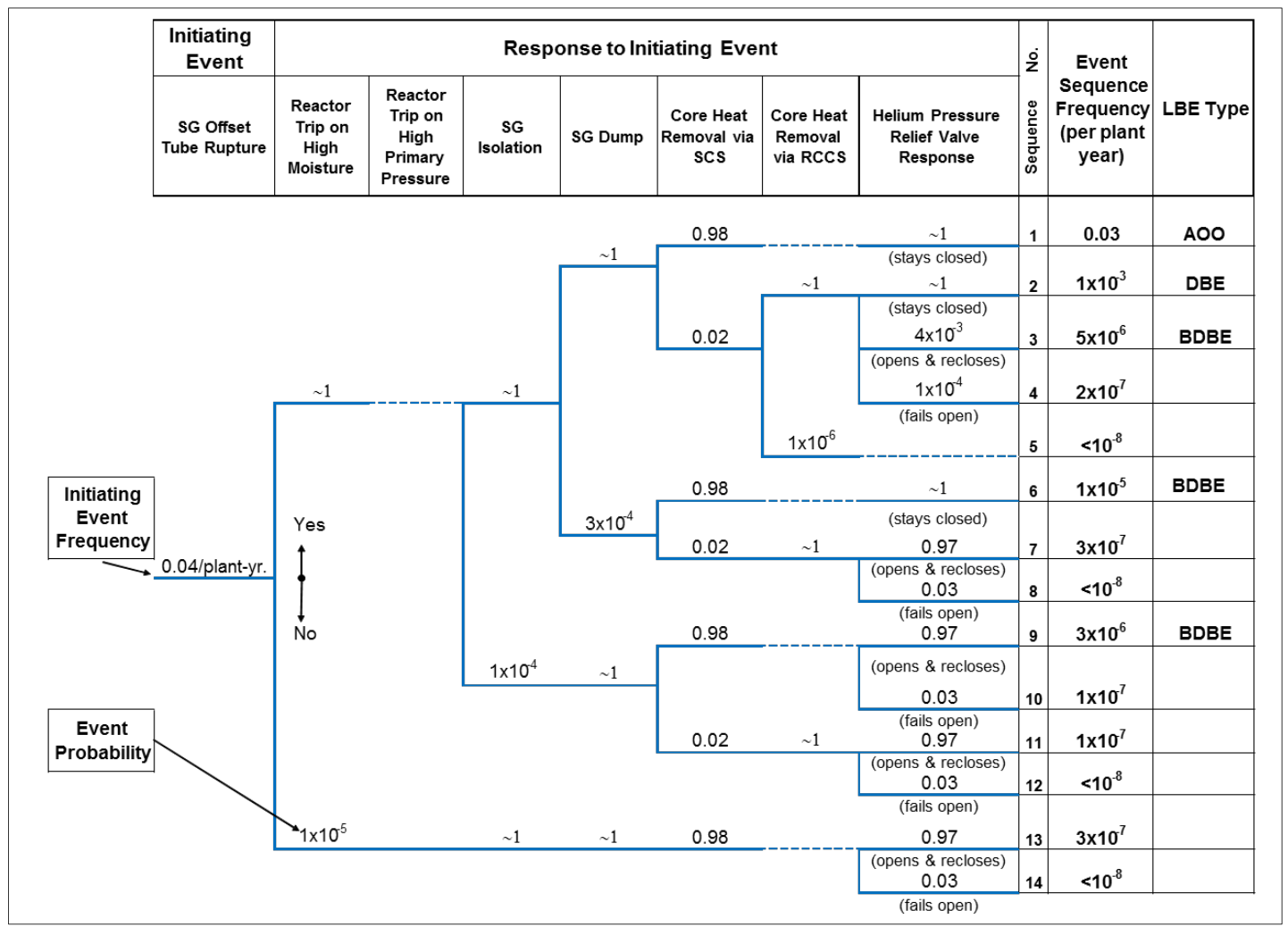

Figure 5-3. Event Tree for MHTGR Steam Generator Tube Rupture

The event trees presented above have been simplified relative to those in the actual PRA in Reference [45] for presentation purposes.

\subsubsection{Definition and Evaluation of MHTGR LBEs}

Event sequence families are used to group together two or more event sequences when the sequences have a common IE, safety function response, and end state. The process of defining event sequence families applies the following considerations:

- The guiding principle is to aggregate event sequences to the maximum extent possible, while preserving the functional impacts of the IE, safety function responses, and end state. Without event sequence families, excessive detail defining IEs and balancing event and fault trees could obscure AOO, DBE, or BDBE event sequence classification and yield an unmanageable set of LBEs. By aggregating sequences into family structures, the event sequence model leaves LBE classification essentially unaffected. This approach prevents the problem in which very detailed event trees may produce an unmanageable number of 
LBEs and the individual event sequence frequencies may be suppressed into the wrong LBE category.

- The safety-function responses are delineated to a necessary and sufficient degree to identify unique challenges to each SSC that performs a given PSF along the event sequence.

- In many cases for a single module plant, there may be only one event sequence in the family.

- For a multi-module plant, event sequence families are used to combine event sequences that involve individual reactor modules independently into a single family of single reactor module event sequences. Event sequences involving multiple reactor modules are always defined as separate LBEs relative to the sequences involving a single module. Accident consequences, where applicable, are evaluated based on the number of reactor modules involved in the release.

- Each event tree IE and PSF response has a corresponding fault tree that delineates the event causes and SSC failure modes that contribute to the frequencies and probabilities of these events.

- Many of the LBEs, especially the AOOs and DBEs with relatively high frequencies have zero consequences. Such LBEs are important to identify for the design because they help define the requirements that must be met by SSCs to affect a safe shutdown and prevent a release. This can be contrasted with typical LWR PRAs that focus on sequences that involve core damage and release of radionuclides from the fuel.

After organizing the event sequences in the detailed PRA into accident families having similar IEs, plant response, and end states, the LBEs in Table 5-1 are defined. 


\section{Table 5-1. LBEs Identified for the MHTGR ${ }^{[44]}$}

\begin{tabular}{|c|c|}
\hline $\begin{array}{c}\text { LBE } \\
\text { Designation }\end{array}$ & LBE Description \\
\hline \multicolumn{2}{|c|}{ Anticipated Operational Occurrences } \\
\hline AOO-1 & $\begin{array}{l}\text { Transient IE with successful reactor trip, continued forced cooling, and intact pressurized HPB involving a } \\
\text { single reactor module. }\end{array}$ \\
\hline AOO-2 & $\begin{array}{l}\text { Loss of Main Loop Cooling IE with successful reactor trip, failure of forced cooling via SCS, and intact } \\
\text { pressurized HPB involving a single reactor module. }\end{array}$ \\
\hline AOO-3 & $\begin{array}{l}\text { Control Rod Withdrawal with successful control rod trip, continued forced cooling with HTS, and intact } \\
\text { pressurized HPB involving a single reactor module. }\end{array}$ \\
\hline AOO-4 & $\begin{array}{l}\text { Small SG Leak with successful reactor trip, SG isolation and dump, forced cooling via SCS, and intact } \\
\text { pressurized HPB involving a single reactor module. }\end{array}$ \\
\hline AOO-5 & $\begin{array}{l}\text { Small HPB Leak with successful reactor trip, continued forced cooling, and successful HPS pump-down, release } \\
\text { of part of circulating activity to reactor building involving a single reactor module. }\end{array}$ \\
\hline \multicolumn{2}{|c|}{ Design Basis Events } \\
\hline DBE-1 & $\begin{array}{l}\text { Loss of offsite power IE and SCS forced cooling, successful reactor trip, passive cooling via RCCS, intact HPB } \\
\text { and no release involving a single reactor module. }\end{array}$ \\
\hline DBE-2 & $\begin{array}{l}\text { Main Loop Transient with Control Rod Trip failure, successful reactor trip via RSS, forced cooling via SCS, intact } \\
\text { HPB, and no release involving a single reactor module. }\end{array}$ \\
\hline DBE-3 & $\begin{array}{l}\text { Control Rod Withdrawal, with successful reactor trip, Main Loop forced cooling failure, forced cooling via SCS, } \\
\text { intact HPB, and no release involving a single reactor module. }\end{array}$ \\
\hline DBE-4 & $\begin{array}{l}\text { Control Rod Withdrawal with successful reactor trip, loss of Main and SCS forced cooling via failures, passive } \\
\text { cooling via RCCS, intact HPB, and no release involving a single reactor module. }\end{array}$ \\
\hline DBE-5 & $\begin{array}{l}\text { Seismic event with loss of offsite power, successful reactor trip, continued forced cooling via Main Loops or } \\
\text { SCS, intact HPB, and no release involving all four reactor modules. }\end{array}$ \\
\hline DBE-6 & $\begin{array}{l}\text { Moderate SG leak with successful reactor trip, SG isolation and dump, forced cooling via SCS, intact HPB, and } \\
\text { no release involving a single reactor module. }\end{array}$ \\
\hline DBE-7 & $\begin{array}{l}\text { Moderate SG leak with successful reactor trip, SG isolation and dump, failure of forced cooling via SCS, intact } \\
\text { HPB, and no release involving a single reactor module. }\end{array}$ \\
\hline DBE-8 & $\begin{array}{l}\text { Moderate SG leak with moisture monitor failure, successful manual reactor trip, SG isolation and dump, } \\
\text { forced cooling via SCS, intact HPB, and no release involving a single reactor module. }\end{array}$ \\
\hline DBE-9 & $\begin{array}{l}\text { Moderate SG leak with successful reactor trip and SG isolation, failure of SG dump, forced cooling via SCS, and } \\
\text { circulating activity release via open primary relief valve to reactor building involving a single reactor module. }\end{array}$ \\
\hline DBE-10 & $\begin{array}{l}\text { Moderate HPB leak with successful reactor trip, continued forced cooling, release of circulating activity, and } \\
\text { lift-off of plateout to reactor building involving a single reactor module. }\end{array}$ \\
\hline DBE-11 & $\begin{array}{l}\text { Small HPB leak with successful reactor trip, failure of forced cooling via Main and SCS Loops, passive cooling } \\
\text { via RCCS, partial release of circulating activity, and delayed fuel release to reactor building involving a single } \\
\text { reactor module. }\end{array}$ \\
\hline \multicolumn{2}{|c|}{ Beyond Design Basis Events } \\
\hline $\begin{array}{l}\text { BDBE-1 } \\
(\mathrm{EPBE}-1)\end{array}$ & $\begin{array}{l}\text { Moderate SG leak with successful reactor trip, delayed SG isolation, SG dump fails, failure of forced cooling via } \\
\text { SCS, HPB relief valve opens due to moisture ingress but fails to reseat, HPB depressurizes to reactor building, } \\
\text { and reactor building vent opens with initial and delayed offsite dose involving a single reactor module. }\end{array}$ \\
\hline $\begin{array}{l}\text { BDBE-2 } \\
(\mathrm{EPBE}-2)\end{array}$ & $\begin{array}{l}\text { Moderate SG leak with successful reactor trip, delayed SG isolation, SG dump fails, successful forced cooling } \\
\text { via SCS, HPB relief valve opens due to moisture ingress but fails to reseat, HPB depressurizes to reactor } \\
\text { building, and reactor building vent opens with initial offsite dose involving a single reactor module. }\end{array}$ \\
\hline $\begin{array}{l}\text { BDBE-3 } \\
\text { (EPBE-3) }\end{array}$ & $\begin{array}{l}\text { Seismic event with small HPB Leaks in all four reactor modules, loss of forced cooling via SCS, release of } \\
\text { circulating activity and delayed fuel release to reactor building, HPB depressurizes, and reactor building vent } \\
\text { opens with offsite dose from release from all four reactor modules. }\end{array}$ \\
\hline
\end{tabular}


Figure 5-4 presents a plot of the LBE frequencies and site boundary doses against the frequencydose criteria used for the MHTGR. Note that the MHTGR used a somewhat different set of frequency-dose criterion than that proposed in the NGNP LBE white paper. The key difference was the classification of events as AOOs with frequencies greater than $0.025 /$ plant-year rather than $10^{-2}$ /plant-year, the use of annual exposure limits in 10 CFR 50 Appendix I rather than 10 CFR 20, and the use of the LBE designator for Emergency Planning Basis Events (EPBEs) instead of BDBE. In addition, both the NGNP and proposed LMP frequency-dose criteria are based on the TEDE, rather than whole body gamma dose as shown for the MHTGR.

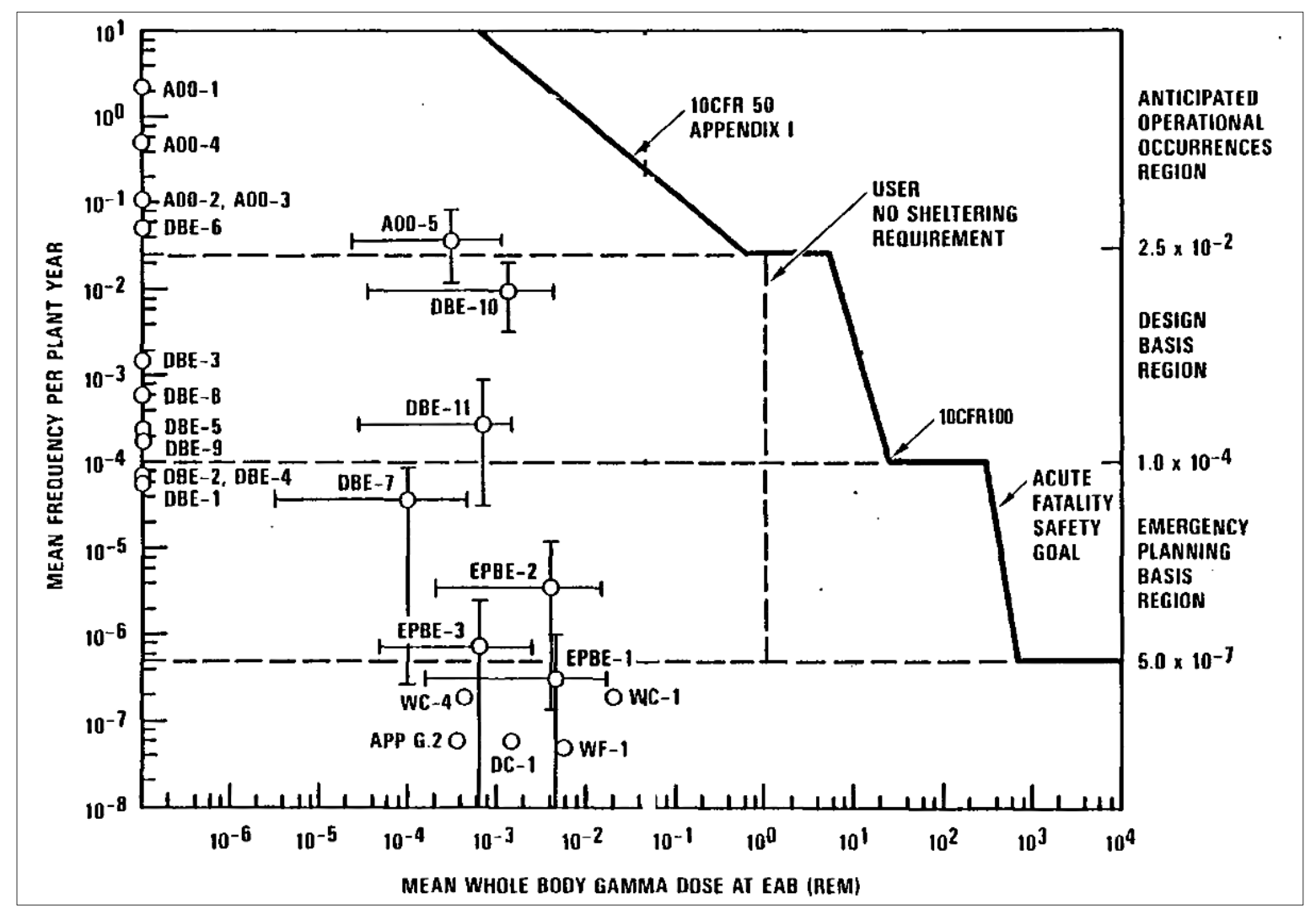

Figure 5-4. Comparison of MHTGR LBE Frequencies and Consequences Frequency-Dose Criteria*

As seen in Figure 5-4, the frequencies and consequences of all the MHTGR LBEs exhibit very large margins against the selected frequency-consequence criteria. Note that in the MHTGR version of this RIPB licensing approach, doses were evaluated in terms of whole-body gamma doses, whereas in the NGNP and proposed LMP approaches, doses are evaluated in terms of TEDE.

*EPBE refers to "Emergency Planning Basis Events," the term used in the MHTGR project to denote BDBE. 


\subsubsection{Definition of MHTGR DBAs for Chapter 15 Evaluation}

DBAs correspond to the traditional off-normal events analyzed in Chapter 15 of the Safety Analysis Report. The approach in this report allows the transition to be made from the traditional deterministic plant response with only SR SSCs responding to DBAs to all SSCs responding to DBEs, so that both the conservative and expected plant behavior are understood.

As noted in Figure 4-8, to begin the design in Task 1, an initial set of prospective LBEs is identified from which to make some of the initial design decisions. The LBEs are then refined in subsequent tasks based on information provided by the initial PRA.

For consistency with current regulatory requirements, DBAs are identified by assuming that only SSCs classified as safety-related are available to perform the PSFs required to meet 10 CFR 50.34 criteria. The DBAs are defined by examining each of the DBEs and BDBEs and noting which SSCs are available and not available to support each PSF. The designer then selects (Task 5 in Figure 4-8) which SSCs are to be classified as safety-related among those available to support each RSF for each DBE. An RSF is one that must be fulfilled to meet the 10 CFR 50.34 dose limits using conservative assumptions. After the SR SSCs are selected, all of the DBEs are reanalyzed with only the SR SSCs responding in a mechanistically conservative manner. Following this process leads to the definition of DBAs for each of the DBEs in Task 6 in Figure 4-8.

DBAs generally do not have the same sequence of events as corresponding DBEs, since the latter consider the expected plant response with all SSCs responding, whether safety-related or not. This means that some of the DBAs would have frequencies that are lower than the DBE frequency cutoff of $10^{-4}$ /plant-year.

As noted previously, each DBE is evaluated to identify which SSCs are available and not available to support each RSF (i.e., those safety functions that must be met to maintain the consequences of the DBE within 10 CFR 50.34 dose limits using conservative assumptions). The safety functions defined for the MHTGR, with the RSFs are shown in Figure 5-5. The development of this figure is based on an exhaustive set of consequence analyses for a wide spectrum of LBEs. One of the RSFs is control core heat removal. To determine which SSCs need to be classified as safety-related requires an examination of each of the DBEs and an analysis of which SSCs are available to support that function for each DBE. 


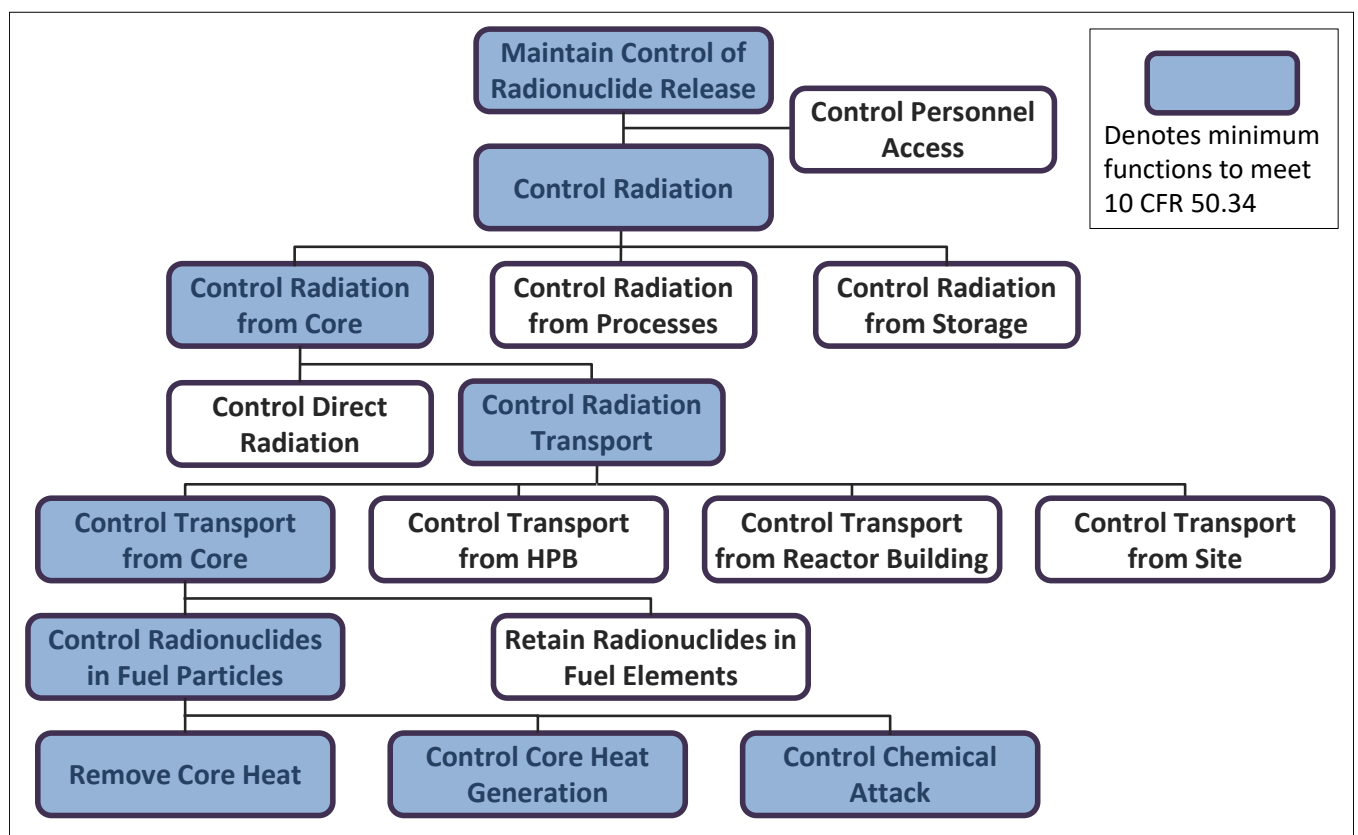

Figure 5-5. MHTGR Safety Functions Including Those Required to Meet 10 CFR 50.34 Limits*

Consider DBE-11 that is defined in the small HPB leak event tree in Figure 5-1. The evaluation of the core heat removal SSCs for that DBE is shown in Table 5-2. For this DBE, there are two sets of SSCs that are capable of providing this RSF, both involving the reactor and the reactor vessel with one transferring heat into the RCCS and the other transferring heat into the passive heat sinks in the reactor cavity of the reactor building.

Table 5-2. Evaluation of Core Heat Removal SSCs for DBE-11

\begin{tabular}{|c|c|}
\hline $\begin{array}{l}\text { SSCs Combinations Capable } \\
\text { of Providing Core Heat Removal }\end{array}$ & Available for DBE-11? \\
\hline $\begin{array}{ll}\text { - } & \text { Reactor } \\
\text { - } & \text { Heat Transport System } \\
\text { - } & \text { Energy Conversion Area (ECA) }\end{array}$ & No \\
\hline $\begin{array}{ll}\text { - } & \text { Reactor } \\
\text { - } & \text { Shutdown Cooling System } \\
\text { - } & \text { Shutdown Cooling Water System (SCWS) }\end{array}$ & No \\
\hline $\begin{array}{ll}\text { - } & \text { Reactor } \\
\text { - } & \text { Reactor Vessel (RV) } \\
\text { - } & \text { Reactor Cavity Cooling System (RCCS) }\end{array}$ & Yes \\
\hline $\begin{array}{ll}\text { - } & \text { Reactor } \\
\text { - } & \text { Reactor Vessel } \\
\text { - } & \text { Reactor Building passive heat sinks (RB) }\end{array}$ & Yes \\
\hline
\end{tabular}

\footnotetext{
* Not shown in this figure is an additional required safety function of "Maintain Core Geometry," which is necessary for Core Heat Removal and Control of Heat Generation.
} 
This evaluation is applied to each of the DBEs to determine which combinations of SSCs are available to support each required safety function. As shown in Table 5-3, there are two options for selecting a set of SR SSCs that are capable of operation for all of the DBEs. The MHTGR design team selected the combination reactor, reactor vessel, and RCCS as SR SSCs. The option that relied on the passive heat sinks in the reactor building as the ultimate heat sink was rejected, as that approach involved the need to address uncertainties regarding concrete degradation, which are removed with a robust and reliable RCCS. This is an example of how deterministic DID considerations had a tangible impact on the selection of SR SSCs and selection of LBEs.

\section{Table 5-3. Evaluation of MHTGR SSCs for Core Heat Removal Safety Function}

\begin{tabular}{|c|c|c|c|c|c|c|c|c|c|c|}
\hline Alternate & & & & & ign Basis & Events & & & & SSCS \\
\hline $\begin{array}{l}\text { Sets of } \\
\text { SSCs }\end{array}$ & DBE 1 & DBE 2 & DBE 3 & DBE 4 & DBE 5 & DBE 6/7 & DBE 8/9 & DBE 10 & DBE 11 & $\begin{array}{c}\text { Classified } \\
\text { as SR? }\end{array}$ \\
\hline $\begin{array}{l}\text { - Reactor } \\
\text { - HTS } \\
\text { - ECA }\end{array}$ & No & No & No & No & No & No & No & No & No & No \\
\hline $\begin{array}{l}\text { - Reactor } \\
\text { - SCS } \\
\text { - SCWS }\end{array}$ & No & Yes & Yes & No & Yes & Yes & Yes & Yes & No & No \\
\hline $\begin{array}{l}\text { - Reactor } \\
\text { - RV } \\
\text { - RCCS }\end{array}$ & Yes & Yes & Yes & Yes & Yes & Yes & Yes & Yes & Yes & Yes \\
\hline $\begin{array}{l}\text { - Reactor } \\
\text { - RV } \\
\text { - RB }\end{array}$ & Yes & Yes & Yes & Yes & Yes & Yes & Yes & Yes & Yes & No \\
\hline
\end{tabular}

When this process is completed for each RSF, it is possible to define DBAs for each of the DBEs where only SR SSCs are assumed to be operable and all of the non-safety-related SSCs are assumed to be failed. The DBAs defined for the MHTGR are shown in Table 5-4. 
Modernization of Technical Requirements

\section{Table 5-4. Definition of Deterministic DBAs for MHTGR}

\begin{tabular}{|c|c|c|c|}
\hline DBE & Design Basis Events & DBA & Design Basis Accidents \\
\hline DBE-1 & $\begin{array}{l}\text { Loss of offsite power IE and SCS forced cooling, successful reactor } \\
\text { trip, passive cooling via RCCS, intact HPB and no release involving } \\
\text { a single reactor module (corresponds to PRA sequence family } \\
\text { with frequency of } 5 \times 10^{-5} / \text { plant-year or about } 1 \times 10^{-5} / \text { reactor-year) }\end{array}$ & DBA-1 & $\begin{array}{l}\text { Loss of Main and SCS forced cooling, successful reactor trip, passive } \\
\text { cooling via RCCS, intact HPB and no release involving a single } \\
\text { reactor module (corresponds to PRA sequence family with } \\
\text { frequency of } 5 \times 10^{-5} / \text { plant-year or about } 1 \times 10^{-5} / \text { reactor-year) }\end{array}$ \\
\hline DBE-2 & $\begin{array}{l}\text { Main Loop Transient with Control Rod Trip failure, successful } \\
\text { reactor trip via RSS, forced cooling via SCS, intact HPB and no } \\
\text { release involving a single reactor module (corresponds to PRA } \\
\text { sequence family with frequency of } 7 \times 10^{-5} / \text { plant-year or about } \\
2 \times 10^{-5} / \text { reactor-year) }\end{array}$ & DBA-2 & $\begin{array}{l}\text { Loss of Main and SCS forced cooling with Control Rod Trip failure, } \\
\text { successful reactor trip via RSS, passive cooling, intact HPB and no } \\
\text { release involving a single reactor module (corresponds to PRA } \\
\text { sequence family with frequency of } 7 \times 10^{-5} / \text { plant-year or about } \\
2 \times 10^{-5} / \text { reactor-year) }\end{array}$ \\
\hline DBE-3 & $\begin{array}{l}\text { Control Rod Withdrawal, with successful reactor trip, Main Loop } \\
\text { forced cooling failure, forced cooling via SCS, intact HPB and no } \\
\text { release involving a single reactor module (corresponds to PRA } \\
\text { sequence family with frequency of } 2 \times 10^{-3} / \text { plant-year or about } \\
5 \times 10^{-4} / \text { reactor-year) }\end{array}$ & DBA-3 & $\begin{array}{l}\text { Control Rod Withdrawal, with successful reactor trip, failure of } \\
\text { forced cooling via Main loops and SCS, passive cooling via RCCS, }\end{array}$ \\
\hline DBE-4 & $\begin{array}{l}\text { Control Rod Withdrawal with successful reactor trip, loss of Main } \\
\text { and SCS forced cooling via failures, passive cooling via RCCS, } \\
\text { intact HPB and no release involving a single reactor module } \\
\text { (corresponds to PRA sequence family with frequency of } \\
7 \times 10^{-5} / \text { plant-year or about } 2 \times 10^{-5} / \text { reactor-year) }\end{array}$ & DBA-4 & $\begin{array}{l}\text { (corresponds to PRA sequence family with frequency of } \\
7 \times 10^{-5} / \text { plant-year or about } 2 \times 10^{-5} / \text { reactor-year) }\end{array}$ \\
\hline DBE-5 & $\begin{array}{l}\text { Seismic event with loss of offsite power, successful reactor trip, } \\
\text { continued forced cooling via Main or SCS, intact HPB and no } \\
\text { release involving all four reactor modules (corresponds to PRA } \\
\text { sequence family with frequency of } 2 \times 10^{-4} / \text { plant-year or } \\
2 \times 10^{-4} / \text { reactor-year) }\end{array}$ & DBA-5 & $\begin{array}{l}\text { Seismic event with loss of offsite power, successful reactor trip, } \\
\text { failure of forced cooling via Main Loops and SCS, passive cooling via } \\
\text { RCCS, intact HPB and no release involving all four reactor modules } \\
\text { (corresponds to PRA sequence family with frequency of } \\
6 \times 10^{-8} / \text { plant-year or about } 6 \times 10^{-8} / \text { reactor-year) }\end{array}$ \\
\hline DBE-6 & $\begin{array}{l}\text { Moderate SG leak with successful reactor trip, SG isolation and } \\
\text { dump, forced cooling via SCS, intact HPB and no release involving } \\
\text { a single reactor module (corresponds to PRA sequence family } \\
\text { with frequency of } 5 \times 10^{-2} / \text { plant-year or about } 1 \times 10^{-2} / \text { reactor-year) }\end{array}$ & DBA-6 & $\begin{array}{l}\text { Moderate SG leak with successful reactor trip and SG isolation, } \\
\text { failure of SG dump, failure of forced cooling via SCS, passive cooling } \\
\text { via RCCS, circulating activity and delayed fuel release via primary } \\
\text { relief valve to reactor building involving a single reactor module } \\
\text { (corresponds to PRA sequence family with frequency of } \\
2 \times 10^{-7} / \text { plant-year or } 5 \times 10^{-8} / \text { reactor-year) }\end{array}$ \\
\hline
\end{tabular}


Modernization of Technical Requirements for Licensing of Advanced Non-Light Water Reactors: Selection and Evaluation of Licensing Basis Events

\section{DBE} Design Basis Events

Design Basis Accidents

Moderate SG leak with successful reactor trip, SG isolation and

DBE-7 dump, failure of forced cooling via SCS, intact HPB and no release involving a single reactor module (corresponds to PRA sequence family with frequency of $4 \times 10^{-5} /$ plant-year or $1 \times 10^{-5} /$ reactor-year) Moderate SG leak with moisture monitor failure, successful manual reactor trip, SG isolation and dump, forced cooling via

DBE-8 SCS, intact HPB and no release involving a single reactor module (corresponds to PRA sequence family with frequency of $4 \times 10^{-5} /$ plant-year)

Moderate SG leak with successful reactor trip and SG isolation, failure of SG dump, forced cooling via SCS, circulating activity

DBE-9 release via open primary relief valve to reactor building involving a single reactor module (corresponds to PRA sequence family with frequency of $2 \times 10^{-4} /$ plant-year)

Moderate HPB leak with successful reactor trip, continued forced cooling, release of circulating activity and lift-off of plateout to

DBE-10 reactor building involving a single reactor module (corresponds to PRA sequence family with frequency of $1 \times 10^{-2} /$ plant-year or about $3 \times 10^{-3} /$ reactor-year)

Small HPB leak with successful reactor trip, failure of forced cooling via Main and SCS Loops; passive cooling via RCCS, partial release of circulating activity and delayed fuel release to reactor building involving a single reactor module (corresponds to PRA sequence family with frequency of $3 \times 10^{-4} /$ plant-year or about $8 \times 10^{-5} /$ reactor-year)
Moderate SG leak with successful reactor trip and SG isolation, via RCCS, circulating activity and delayed fuel release via primary relief valve to reactor building involving a single reactor module (corresponds to PRA sequence family with frequency of $<10^{-8} /$ plantyear or $<10^{-8} /$ reactor-year)
Moderate HPB leak with successful reactor trip, failure of forced cooling via Main loops and SCS, passive cooling via RCCS, release of circulating activity, delayed fuel release, and lift-off of plateout to reactor building involving a single reactor module (corresponds to PRA sequence family with frequency of $6 \times 10^{-8} /$ plant-year or about $1.5 \times 10^{-8} /$ reactor-year)

Small HPB leak with successful reactor trip, failure of forced cooling via Main and SCS, partial release of circulating activity and delayed

DBA-11 fuel release to reactor building involving a single reactor-module (corresponds to PRA sequence family with frequency of $<10^{-8} /$ plantyear or $<10^{-8} /$ reactor-year) 
In the course of defining these DBAs, the MHTGR design team classified the following additional SSCs as safety-related for the other shaded functions in Figure 5-5: the RCS and RSS reactor trip systems, the moisture monitors, and SSCs necessary to ensure successful isolation of a leaking steam generator, but not the SG dump system. There are three DBEs for which the corresponding DBA is the same because the RSFs are performed by the SR SSCs for that function. For DBE-3 and DBE-4, the same DBA is defined. Also, for DBE-6, DBE-7, DBE-8, and DBE-9, the same DBA is defined. Thus, there are fewer DBAs than DBEs. This stems from the fact that some of the DBEs have successful operation of one or more non-safety SSCs to perform an RSF, whereas DBAs only have SSR SSCs assumed to be operational for such functions.

Each of the DBAs are then included in Chapter 15 of the license application and are analyzed using conservative assumptions and demonstrated to meet 10 CFR 50.34 dose limits.

\subsection{Example LBE Development for PRISM}

In this section, some examples from the PRISM PRA ${ }^{[72]}$ are used to illustrate some of the key tasks in the LBE definition process of Figure 4-8. The PRISM example presented in this section is a simplified event tree for a loss of forced flow event. Examples of AOOs, DBEs, and BDBEs are also taken from the PRISM PRA, and the process of selecting options for SR SSCs is demonstrated. The LBE development for PRISM presented in this section was subsequently refined in an LMP table top exercise documented in Reference [78]. These PRISM examples benefit from a recently completed PRA upgrade that was performed for PRISM, which served as one of the pilot PRAs for the Advanced non-LWR Trial Use PRA Standard. ${ }^{[36]}$ More examples from the PRISM PRA are included in a companion LMP report on PRA development for advanced non-LWRs.

The PRISM reactor plant participated in a table top execution of the LMP methodology subsequent to the preparation of this report. A more expanded execution of the LMP methodology for this reactor technology can be found in Reference [78].

\subsubsection{Example Event Tree Development}

The PRISM plant is comprised of two reactor modules whose reactors are of the pool-type liquid-metal-cooled fast reactor similar in design to EBR-2. The PRISM PRA included a systematic search for IEs and included the development and quantification of the frequencies and consequences for the following categories of IEs:

- Balance-of-plant (BOP)/loss of heat sink (LOHS) faults

- Intermediate heat exchanger bypass leak

- Intermediate Heat Transport System leak

- Loss of offsite power

- Loss of primary forced flow (LOF)

- Nuclear steam supply system transients 
- Turbine/BOP transient faults

- Steam generator tube rupture

- Transient overpower

Event sequence models were developed based on the challenges that the IEs placed on radionuclide release barriers. Plant response analysis includes assessments of structural integrity, thermal-hydraulic sodium system temperatures, and fuel performance. For sequences involving any damage to fuel cladding to the core assemblies or spent fuel stored in the vessel, a mechanistic source term analysis calculates the radionuclide transport from the fuel to the sodium coolant hot pool, to the cover gas space, through leakage paths in the vessel, and through leakage paths in containment into the environment.

Figure 5-6 provides a simplified LOF event tree from a single electromagnetic (EM) pump failure. With conservative estimates for various failure modes across the eight EM pumps across the two reactor units, the total frequency of LOF from a single pump is about 1/plant-year.

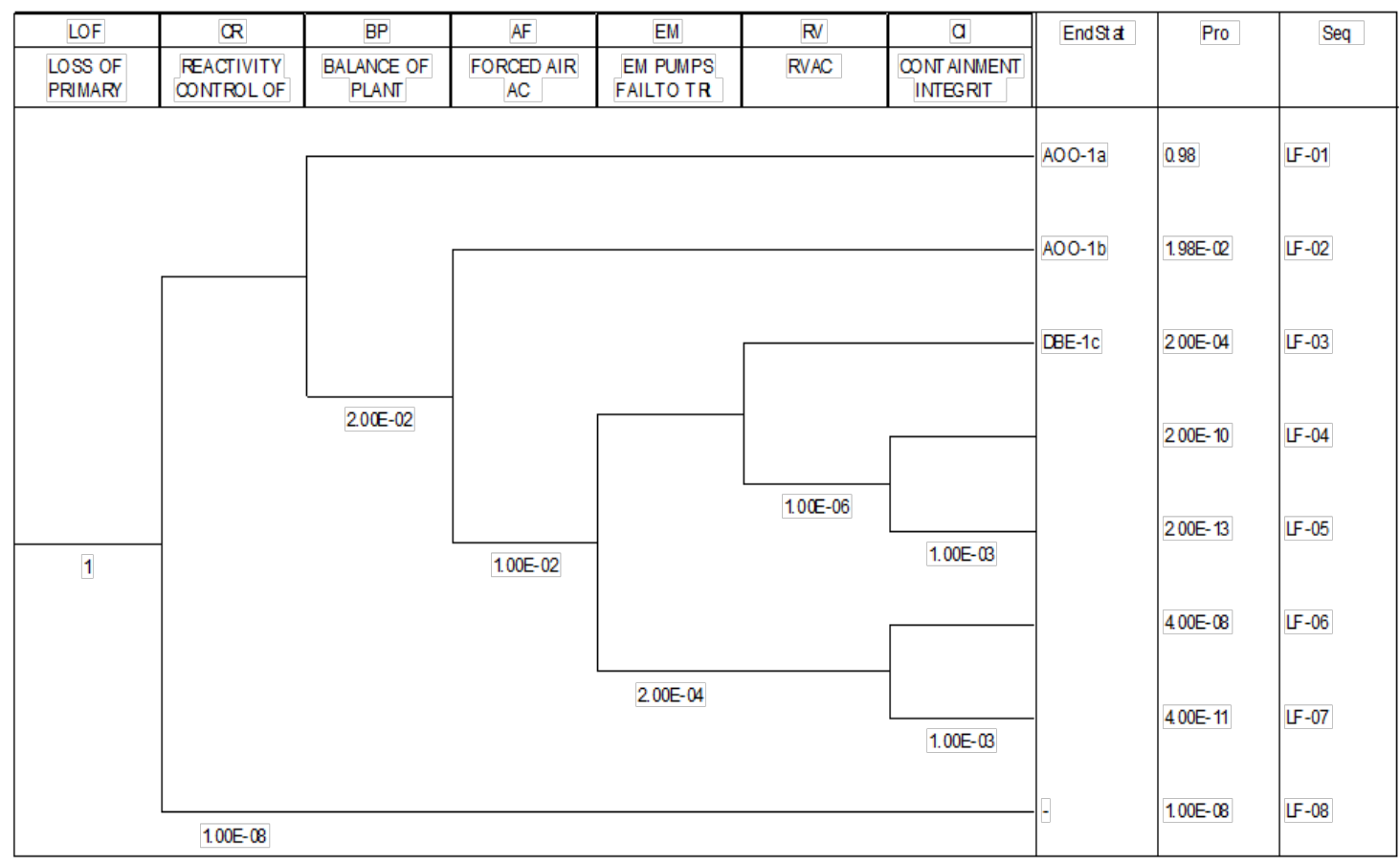

Figure 5-6. Event Tree for Loss of Flow in a Single EM Pump

LOF is immediately detected by redundant pressure sensors at discharge points for each of the EM pumps, which triggers a scram in the Reactor Protection System (RPS). Because of redundancy in the RPS architecture, the dominant scram failure mode is a common cause software failure of digital instrumentation and controls, assessed as $1 \times 10^{-4}$ per demand. Failure of RPS is accommodated by a Diverse Protection System, which is a digital system on a completely independent platform, also dominated by a common cause software demand failure. 
Overall, the failure of scram following the LOF IE has an event sequence frequency below the range of BDBE.

Once the reactivity control function is performed by successful control rod scram, the safety function of interest is decay heat removal. The first system satisfying this function is the normal compliment of feedwater and condensate systems to remove decay heat through the steam generator. This event represents AOO-1a. In the event that these BOP systems fail, decay heat could be removed by manual actuation of a fan that circulates air around the shell of the steam generator. This forced air cooling mode of the Steam Generator Auxiliary Cooling System (SGACS) can remove all decay heat transferred by the intermediate sodium cooling loop to the steam generator. This is event AOO-1b.

On failure of both the BOP and SGACS active systems, decay heat removal can be accomplished by the Reactor Vessel Auxiliary Cooling System (RVACS), which consists of pathways for air to circulate around the outside of the containment vessel. The RVACS, which is a set of plant structures rather than a system, is capable of removing decay heat loads entirely with passive natural air circulation. To fulfill this function, the EM pumps that drive primary and intermediate coolant need to have successfully tripped. Each of the pumps is self-cooled, meaning that its full workload is transferred to the sodium coolant. This event sequence in which an LOF IE is followed by failures of BOP cooling and the backup SGACS fan is DBE-1c.

The LOF event tree contains several events where fuel damage and thus release is possible. An LF-04 event is the result when RVACS passive flow conditions are degraded in such a manner that temperatures in the vessel climb and challenge fuel integrity criteria. Passive reliability analysis for RVACS justifies that many off-normal structural conditions are needed to degrade RVACS performance so severely, resulting in an overall reliability on the order of $1 \times 10^{-6}$ per demand. After fuel damage, a very small radionuclide release in this event occurs as the result of design leakage out of the vessel and then out of the containment. The frequency of this event sequence has been judged to be below the range of BDBEs.

The LOF event tree had yet another unlikely sequence postulating fuel damage. This is the event (LF-06) in which BOP cooling and SGACS fail, and there is a failure of a minimum number of EM pumps to trip. If the EM pumps are allowed to continue running, the heat load on the sodium coolant is larger than the capacity of RVACS heat removal, resulting in eventual fuel damage. Similar to LF-04, this event results in a small release through design leakage paths, as calculated by a mechanistic source term analysis. This event is also well below the range of BDBEs.

The collection of event sequences following the LOF initiator is typical for the decay heat removal function following any IE in the PRISM plant.

\subsubsection{Definition and Evaluation of PRISM LBES}

The AOOs and DBE identified in the LOF event tree above are listed in Table 5-5, alongside examples of BDBEs identified from other event trees not discussed here. Figure 5-7 provides a plot of the LBE frequencies and site boundary doses against the frequency-dose criteria used for 
PRISM. Similar to the conclusion for MHGTR, the frequencies and consequences of all the PRISM LBEs exhibit very large margins against the selected LMP frequency-consequence criteria. This conclusion is expected to remain true when the full list of events studied in the PRA is added.

\section{Table 5-5. LBEs Identified for the PRISM Loss of Flow Event Tree}

\begin{tabular}{|ll|}
\hline \multicolumn{2}{|c|}{ LBE } \\
Designation
\end{tabular}

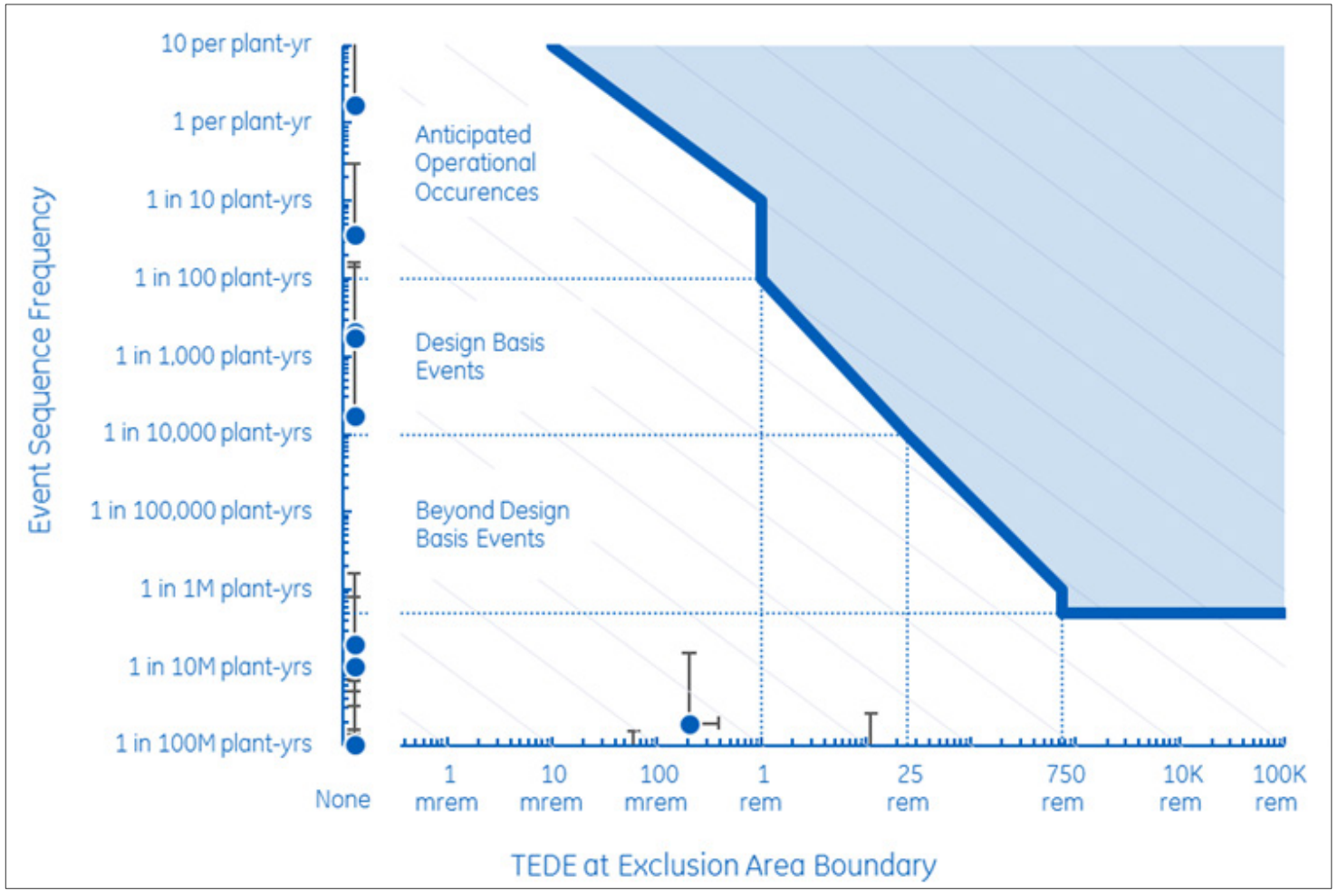

Figure 5-7. Comparison of PRISM LBE Frequencies and Consequences and LMP FrequencyConsequence Targets 


\subsubsection{Example Definition of PRISM DBAs}

With the events taken from the Internal Events At-Power PRA for PRISM, there are a limited number of events that fall in the DBE range. Of these events, nearly all fit the pattern of some sort of plant trip, followed by failures of multiple active cooling systems, leaving the decay heat removal function to the passive air-cooling with RVACS, as in the DBE example discussed above.

For DBE-1c, which is a typical decay heat removal challenge, the combinations of SSCs needed to meet dose release limits set by 10 CFR 50.34 are provided in Table 5-6. The table shows that there are three primary ways to remove decay heat and avoid fuel damage: BOP cooling, forcedair cooling through SGACS, or passive air cooling through RVACS. A fourth option is provided for completeness, leveraging the mechanistic source term analysis that shows that given fuel damage from lack of cooling, the dose release could meet 10 CFR 50.34 limits if both the vessel head and containment perform their confinement function successfully.

Table 5-6. Evaluation of SSCs Limiting Dose Release for PRISM DBE-1c

\begin{tabular}{|c|c|}
\hline $\begin{array}{l}\text { SSCs Combinations Capable of } \\
\text { Meeting } 10 \text { CFR 50.34 Dose Limits }\end{array}$ & Available for DBE $1 c$ ? \\
\hline $\begin{array}{ll}\text { - } & \text { Reactor Vessel } \\
\text { - } & \text { Control Rod Scram } \\
\text { - } & \text { BOP Cooling } \\
\text { - } & \text { RVACS (passive air cooling) }\end{array}$ & No \\
\hline $\begin{array}{ll}\text { - } & \text { Reactor Vessel } \\
\text { - } & \text { Control Rod Scram } \\
\text { - } & \text { SGACS Cooling }\end{array}$ & No \\
\hline $\begin{array}{ll}\text { - } & \text { Reactor Vessel } \\
\text { - } & \text { Control Rod Scram } \\
\text { - } & \text { RVACS Passive Air-Cooling }\end{array}$ & Yes \\
\hline $\begin{array}{ll}\text { - } & \text { Reactor Vessel } \\
\text { - } & \text { Control Rod Scram } \\
\text { - } & \text { No Decay Heat Removal (fuel damage) } \\
\text { - } & \text { Vessel Head } \\
\text { - } & \text { Containment } \\
\end{array}$ & Yes \\
\hline
\end{tabular}

It should be noted that these PRISM example DBAs were developed by taking a pre-existing PRA that was originally focused on the identification of event sequences with releases and consequences. Had the PRA been originally developed for the purpose of identifying LBEs, the challenges to the safety functions that do not involve a release would have been more fully developed resulting in additional DBAs.

A more complete set of DBEs and corresponding DBAs for PRISM is shown in Table 5-7. 
Modernization of Technical Requirements

for Licensing of Advanced Non-Light Water Reactors:

Selection and Evaluation of Licensing Basis Events

\section{Table 5-7. Definition of Deterministic DBAs for PRISM}

DBE Design Basis Events

Spurious control rod withdrawal with pre-existing rod-stop error and failure of BOP

DBE-1 cooling; RPS shuts down reactor and active SGACS removes decay heat involving one reactor module Spurious control rod withdrawal with pre-existing rod-stop error and failure of BOP

DBE-2 cooling and SGACS; RPS shuts down reactor, the EM pumps trip, and passive RVACS removes decay heat, supplemented by passive mode of SGACS involving one reactor module

Spurious control rod withdrawal with pre-existing rod-stop error and failure of BOP

DBE-3 cooling and SGACS; RPS shuts down reactor, the EM pumps trip, and passive RVACS removes decay heat involving one reactor module

SG tube rupture is detected and suppressed by sodium-water reaction detection

DBE-4 equipment, RPS shuts down the reactor, and active SGACS removes decay heat involving one reactor module

A general transient with failure of BOP cooling and forced SGACS; RPS shuts down the

DBE-5 reactor, the EM pumps trip, and passive RVACS removes decay heat supplemented by passive mode of SGACS involving both reactor modules

A general transient with failure of BOP cooling and all modes of SGACS; RPS shuts down

DBE-6 the reactor, the EM pumps trip, and passive RVACS removes decay heat involving both reactor modules

A general transient with failure of the intermediate sodium coolant loop; RPS shuts down

DBE-7 the reactor, the EM pumps trip, and passive RVACS removes decay heat involving both reactor modules

A plant-centered loss of offsite power with failure of backup power to forced SGACS; RPS

DBE-8 shuts down the reactor and passive RVACS removes decay heat involving both reactor modules

A major hurricane causes both a loss of offsite power and an off-normal condition for

DBE-9 RVACS; RPS shuts down the reactor and passive RVACS removes decay heat under storm conditions involving both reactor modules
Spurious control rod withdrawal with pre-existing rod-stop error and failure of BOP cooling and forced SGACS cooling;

DBA-1 RPS shuts down the reactor and passive RVACS removes decay heat, including the extra power generated during the transient overpower involving one reactor module

SG tube rupture with failure of sodium-water reaction detection and suppression equipment, which disables all DBA-2 cooling modes through the intermediate loop; RPS shuts down the reactor and passive RVACS removes decay heat involving one reactor module

A general transient with failure of BOP cooling and forced SGACS; RPS shuts down the reactor, the EM pumps trip and passive RVACS removes decay heat involving both reactor modules
A major hurricane causes both a loss of offsite power and an off-normal condition for RVACS; RPS shuts down the reactor and passive RVACS removes decay heat under storm conditions involving both reactor modules 


\subsection{LBE Development for Molten Salt Reactors}

\subsubsection{LBEs for Fluoride Salt-Cooled High Temperature Reactors}

The University of California, Berkeley, Massachusetts Institute of Technology, and University of Wisconsin-Madison hosted a series of workshops during 2012 under a DOE-sponsored Integrated Research Project to review technical and licensing issues for FHRs. The focus of the first workshop whose results are documented in Reference [77] was to identify key development goals for FHRs, including the major technical characteristics that differentiate FHRs from other power reactor technologies, the major systems and subsystems expected to be used in FHRs, high-level functional requirements for these systems and subsystems, and LBEs that should be considered in FHR design and licensing.

The first chapter in Reference [75] provides an overview of the Integrated Research Project and the FHR technology, as well as a high-level discussion of the FHR licensing strategy. The second chapter lays out the FHR system decomposition and the safety design criteria. The third chapter reviews the candidate materials, including fuels, structural materials, and fluids that would be used in FHRs. The fourth chapter focuses on the selection of FHR LBEs and first identifies the existing NRC precedent for LBE identification for existing light-water reactor technology. The white paper provides a preliminary set of bounding LBEs and a detailed discussion on the LBE identification logic. Appendix A identifies the major system and subsystem functional requirements for FHRs.

This example of LBE development occurred prior to the initiation of the LMP but benefitted from earlier work on the MHTGR that shared key elements of the LMP methodology.

\subsubsection{LBEs for Molten Salt Reactor Experiment}

To demonstrate how the LBE selection process described in this report would be applied to an advanced reactor design, this approach was applied to the MSRE, a test reactor designed, constructed, and operated at the Oak Ridge National Laboratory in the 1960s. The MSRE design was chosen for this work because (1) it is a non-proprietary MSR reference design, (2) extensive design information is available, (3) the design information includes extensive separate effects and integrated testing, and (4) information is available from five years of documented testing and operations experience. The MSRE was a single-region circulating liquid salt-fueled and cooled thermal-spectrum reactor. It was capable of producing heat at a maximum rate of $7.34 \mathrm{MW}$. The fuel was UF4 in a carrier salt of $\mathrm{LiF}-\mathrm{BeF}_{2}-\mathrm{ZrF}_{4}$, and the operating temperature of the fuel salt loop averaged approximately $650^{\circ} \mathrm{C}\left(1,200^{\circ} \mathrm{F}\right)$, with a hot leg temperature of about $657^{\circ} \mathrm{C}$ and a cold leg temperature of about $624^{\circ} \mathrm{C}$.

Reference [76] describes how existing MSRE hazard reports and safety analyses were used to investigate how the early steps of the process for selecting LBEs would be performed for an advanced non-LWR reactor design. The report evaluates the event sequences associated with three different IEs to provide a preliminary albeit incomplete set of LBEs for the MSRE. The analysis presented in this study does not lead to selection of DBAs or SR SSCs because an exhaustive hazard analysis does not exist for MSRE and because the results of the steps 
performed were completed only to demonstrate the applicability of the LMP approach to LBE selection to this type of advanced reactor.

This work demonstrated that the LMP methodology was effective in defining LBEs for MSRs and showed how the use of a PHA is an effective means of developing the knowledge base for development of PRA models.

\subsection{Example LBE Development in LMP Table Top Exercises}

Additional examples of LBE selection and evaluation following the LMP methodology have been and are in the process of being developed in LMP table top exercises that are being performed to pilot the LMP methodology. To date, two table tops have been completed. The first was on the X-Energy pebble bed HTGR whose results are presented in Reference [77]. The second completed table top was performed on the PRISM that extends the examples presented in this report and is documented in Reference [78]. Lessons learned from these table tops have already been incorporated into the industry guidance in NEI 18-04 and the supporting reports. In addition, these table tops exercise other tasks of the LMP methodology beyond LBE selection including SSC safety classification and some aspects of DID evaluation. 


\subsection{LMP LBE SELECTION APPROACH SUMMARY}

In summary, the regulatory precedents were reviewed to identify guidance for selecting LBEs for advanced non-LWRs. Example approaches for selecting LBEs were identified from DOE's NGNP project and NUREG-1860. Similar approaches were also identified with the Yucca Mountain Preclosure Safety Analysis and the U.K. SAPs. The regulatory precedent review benefitted from more recent developments since the NGNP LBE white paper was developed and provided additional insights into the formulation of frequency-consequence criteria for evaluating the risk significance of selected LBEs.

Based on the review of the regulatory precedents and consistent with LMP objectives, a set of LBE selection attributes were developed. To meet these attributes, the LBE selection approach shall be:

- Systematic and reproducible

- Reasonably complete

- Provide timely input to design decisions

- Risk-informed and performance-based

- Reactor technology-inclusive

- Consistent with applicable regulatory requirements

An approach to identifying LBEs for the LMP was selected using the NGNP LBE as a starting point. An earlier version of the NGNP approach was successfully applied in the MHTGR project, which included the development of a conceptual design of an MHTGR plant consisting of four reactor modules, a multi-module PRA, a Preliminary Safety Information Document, and several topical reports that described the derivation of LBEs and the selection of SR SSCs. This information was subjected to a preliminary review by NRC staff and supporting national laboratories. The risk-informed and performance-based approach reflected in this MHTGR case was subsequently refined in the Exelon PBMR and NGNP projects.

With the benefits of an expanded regulatory precedent review and feedback from NRC and ACRS on the NGNP and supporting projects, a number of refinements are proposed in the LMP LBE selection approach. These refinements have benefited from developing example LBE selections for the PRISM to ensure the selected approach is reactor technology-inclusive. The refinements include:

- Refinement to the F-C Target for evaluating the risk significance of individual LBEs

- Addition of a risk metric and associated performance goals to evaluate the integrated risks of the multi-module advanced non-LWR plant, beyond the two QHO risk metrics used in the NGNP approach. The additional metric addresses a goal for ensuring that the annual dose limits in 10 CFR 20 are met. 
Beyond these changes, the LBE approach that is proposed is consistent with that originally developed for the MHTGR and subsequently refined during the Exelon PBMR and NGNP projects. It is the view of the LMP team that the LBE approach described here has the LBE selection attributes listed above and the capability to derive a set of LBEs that will be necessary and sufficient for the design and licensing of advanced non-LWR plants.

The proposed approach is systematic and reproducible. It has been demonstrated to be reactor technology-inclusive using examples from several distinctly different reactor types, a modular HTGR, a pool-type liquid-metal-cooled fast reactor, PRISM, and two variations of reactors using molten salt as coolant including the FHR and the MSRE. It is risk-informed because it employs an appropriate balance of deterministic and probabilistic inputs and is consistent with the principles of DID. The approach is performance-based at the plant level through the use of a F-C Target that can be used to focus the attention of the designer and those reviewing the design and related operational programs to the most risk significant events and possible means to address those events. This process leads to RIPB practices at lower-tier activities including designspecific principle design criteria development, RIPB SSC classification and capability targets, and a well-structured methodology for DID adequacy evaluation. While addressing the fundamental differences between advanced non-LWRs and LWRs, the approach is designed to address applicable regulatory requirements and has the capability to support successful future license applications that reflect the NRC Safety Goal and Advanced Reactor policies. 


\subsection{GLOSSARY OF TERMS}

\section{LMP Term Acronym}

Terms Associated with Functions

\begin{tabular}{|c|c|c|c|}
\hline $\begin{array}{l}\text { Fundamental } \\
\text { Safety Function }\end{array}$ & FSF & $\begin{array}{l}\text { Safety functions common to all reactor technologies and designs; includes control } \\
\text { heat generation, control heat removal and confinement of radioactive material }\end{array}$ & IAEA-TECDOC-1570 \\
\hline $\begin{array}{l}\text { PRA Safety } \\
\text { Function }\end{array}$ & PSF & $\begin{array}{l}\text { Reactor design specific SSC functions modeled in a PRA that serve to prevent and/or } \\
\text { mitigate a release of radioactive material or to protect one or more barriers to } \\
\text { release. In ASME/ANS-Ra-S-1.4-2013 these are referred to as "safety functions." } \\
\text { The modifier PRA is used in the LMP GD to avoid confusion with safety functions } \\
\text { performed by Safety-Related SSCs. }\end{array}$ & $\begin{array}{l}\text { LMP, } \\
\text { ASME/ANS-Ra-S-1.4- } \\
2013\end{array}$ \\
\hline $\begin{array}{l}\text { Prevention } \\
\text { Function }\end{array}$ & -- & $\begin{array}{l}\text { An SSC function that, if fulfilled, will preclude the occurrence of an adverse state. } \\
\text { The reliability of the SSC in the performance of such functions serves to reduce the } \\
\text { probability of the adverse state. }\end{array}$ & LMP \\
\hline $\begin{array}{l}\text { Mitigation } \\
\text { Function }\end{array}$ & -- & $\begin{array}{l}\text { An SSC function that, if fulfilled, will eliminate or reduce the consequences of an } \\
\text { event in which the SSC function is challenged. The capability of the SSC in the } \\
\text { performance of such functions serves to eliminate or reduce any adverse } \\
\text { consequences that would occur if the function were not fulfilled. }\end{array}$ & LMP \\
\hline $\begin{array}{l}\text { Required Safety } \\
\text { Function }\end{array}$ & RSF & $\begin{array}{l}\text { A PRA Safety Function that is required to be fulfilled to maintain the consequence of } \\
\text { one or more DBEs or the frequency of one or more high-consequence BDBEs inside } \\
\text { the F-C Target }\end{array}$ & LMP \\
\hline $\begin{array}{l}\text { Required } \\
\text { Functional Design } \\
\text { Criteria }\end{array}$ & RFDC & $\begin{array}{l}\text { Reactor design-specific functional criteria that are necessary and sufficient to meet } \\
\text { the RSFs }\end{array}$ & LMP \\
\hline $\begin{array}{l}\text { Safety-Related } \\
\text { Design Criteria }\end{array}$ & SRDC & $\begin{array}{l}\text { Design criteria for SR SSCs that are necessary and sufficient to fulfill the RFDCs for } \\
\text { those SSCs selected to perform the RSFs }\end{array}$ & LMP \\
\hline \multicolumn{4}{|c|}{ Terms Associated with Licensing Basis Events } \\
\hline $\begin{array}{l}\text { Anticipated } \\
\text { Operational }\end{array}$ & AOO & $\begin{array}{l}\text { Anticipated event sequences expected to occur one or more times during the life of } \\
\text { a nuclear power plant, which may include one or more reactor modules. Event }\end{array}$ & LMP \\
\hline
\end{tabular}




\begin{tabular}{|c|c|c|c|}
\hline LMP Term & Acronym & Definition & Source \\
\hline Occurrence & & $\begin{array}{l}\text { sequences with mean frequencies of } 1 \times 10^{-2} / \text { plant-year and greater are classified as } \\
\text { AOOs. AOOs take into account the expected response of all SSCs within the plant, } \\
\text { regardless of safety classification. }\end{array}$ & \\
\hline $\begin{array}{l}\text { Design Basis } \\
\text { Event }\end{array}$ & DBE & $\begin{array}{l}\text { Infrequent event sequences that are not expected to occur in the life of a nuclear } \\
\text { power plant, which may include one or more reactor modules, but are less likely } \\
\text { than AOOs. Event sequences with mean frequencies of } 1 \times 10^{-4} / \text { plant-year to } 1 \times 10^{-} \\
2 / \text { plant-year are classified as DBEs. DBEs take into account the expected response } \\
\text { of all SSCs within the plant regardless of safety classification. The objective and } \\
\text { scope of DBEs form the safety design basis of the plant. }\end{array}$ & LMP \\
\hline $\begin{array}{l}\text { Beyond Design } \\
\text { Basis Event }\end{array}$ & BDBE & $\begin{array}{l}\text { Rare event sequences that are not expected to occur in the life of a nuclear power } \\
\text { plant, which may include one or more reactor modules, but are less likely than a } \\
\text { DBE. Event sequences with frequencies of } 5 \times 10^{-7} / \text { plant-year to } 1 \times 10^{-4} / \text { plant -year } \\
\text { are classified as BDBEs. BDBEs take into account the expected response of all SSCs } \\
\text { within the plant regardless of safety classification. }\end{array}$ & LMP \\
\hline $\begin{array}{l}\text { Design Basis } \\
\text { Accident }\end{array}$ & DBA & $\begin{array}{l}\text { Postulated accidents that are used to set design criteria and performance objectives } \\
\text { for the design of Safety-Related SSCs. DBAs are derived from DBEs based on the } \\
\text { capabilities and reliabilities of Safety-Related SSCs needed to mitigate and prevent } \\
\text { accidents, respectively. DBAs are derived from the DBEs by prescriptively assuming } \\
\text { that only SR SSCs classified are available to mitigate postulated accident } \\
\text { consequences to within the } 10 \text { CFR } 50.34 \text { dose limits. }\end{array}$ & LMP \\
\hline $\begin{array}{l}\text { Licensing Basis } \\
\text { Event }\end{array}$ & LBE & $\begin{array}{l}\text { The entire collection of event sequences considered in the design and licensing } \\
\text { basis of the plant, which may include one or more reactor modules. LBEs include } \\
\text { AOOs, DBEs, BDBEs, and DBAs. }\end{array}$ & LMP \\
\hline $\begin{array}{l}\text { Frequency- } \\
\text { Consequence } \\
\text { Target }\end{array}$ & F-C Target & $\begin{array}{l}\text { A target line on a frequency-consequence chart that is used to evaluate the risk } \\
\text { significance of LBEs and to evaluate risk margins that contribute to evidence of } \\
\text { adequate defense-in-depth }\end{array}$ & LMP \\
\hline $\begin{array}{l}\text { Risk-Significant } \\
\text { LBE }\end{array}$ & -- & $\begin{array}{l}\text { An LBE whose frequency and consequence meet a specified risk significance } \\
\text { criterion. In the LMP framework, an AOO, DBE, or BDBE is regarded as risk- } \\
\text { significant if the combination of the upper bound ( } 95^{\text {th }} \text { percentile) estimates of the } \\
\text { frequency and consequence of the LBE are within } 1 \% \text { of the F-C Target AND the } \\
\text { upper bound 30-day TEDE dose at the EAB exceeds } 2.5 \text { mrem. }\end{array}$ & LMP \\
\hline
\end{tabular}


Modernization of Technical Requirements

\begin{tabular}{|c|c|c|c|}
\hline LMP Term & Acronym & Definition & Source \\
\hline \multicolumn{4}{|c|}{ Terms Associated with Plant Design and Structures, Systems, and Components } \\
\hline $\begin{array}{l}\text { Design Basis } \\
\text { External Hazard } \\
\text { Level }\end{array}$ & DBEHL & $\begin{array}{l}\text { A design specification of the level of severity or intensity of an external hazard for } \\
\text { which the Safety-Related SSCs are designed to withstand with no adverse impact on } \\
\text { their capability to perform their RSFs }\end{array}$ & LMP \\
\hline Plant & & $\begin{array}{l}\text { The collection of site, buildings, radionuclide sources, and SSCs seeking a single } \\
\text { design certification or one or more operating licenses under the LMP framework. } \\
\text { The plant may include a single reactor unit or multiple reactor modules as well as } \\
\text { non-reactor radionuclide sources. }\end{array}$ & LMP \\
\hline $\begin{array}{l}\text { Multi-Reactor } \\
\text { Module Plant }\end{array}$ & -- & $\begin{array}{l}\text { A plant comprising multiple reactor modules that are designed and constructed } \\
\text { using a modular design approach. Modular design means a nuclear power plant } \\
\text { that consists of two or more essentially identical nuclear reactors (modules) and } \\
\text { each reactor module is a separate nuclear reactor capable of being operated } \\
\text { independent of the state of completion or operating condition of any other reactor } \\
\text { module co-located on the same site, even though the nuclear power plant may } \\
\text { have some shared or common systems. }\end{array}$ & $\begin{array}{l}\text { Multi-module plant } \\
\text { adapted from } \\
\text { ASME/ANS-Ra-S-1.4- } \\
\text { 2013, modular design } \\
\text { from 10CFR52.1 }\end{array}$ \\
\hline $\begin{array}{l}\text { Safety-Related } \\
\text { SSCs }\end{array}$ & SR SSCS & $\begin{array}{l}\text { SSCs that are credited in the fulfillment of RSFs and are capable to perform their } \\
\text { RSFs in response to any Design Basis External Hazard Level }\end{array}$ & LMP \\
\hline $\begin{array}{l}\text { Non-Safety- } \\
\text { Related with } \\
\text { Special } \\
\text { Treatment SSCs }\end{array}$ & $\begin{array}{l}\text { NSRST } \\
\text { SSCS }\end{array}$ & $\begin{array}{l}\text { Non-safety-related SSCs that perform risk-significant functions or perform functions } \\
\text { that are necessary for defense-in-depth adequacy }\end{array}$ & LMP \\
\hline $\begin{array}{l}\text { Non-Safety- } \\
\text { Related with No } \\
\text { Special } \\
\text { Treatment SSCs }\end{array}$ & NST SSCs & $\begin{array}{l}\text { All SSCs within a plant that are neither Safety-Related SSCs nor Non-Safety-Related } \\
\text { SSCs with Special Treatment SSCs }\end{array}$ & LMP \\
\hline $\begin{array}{l}\text { Risk-Significant } \\
\text { SSC }\end{array}$ & -- & $\begin{array}{l}\text { An SSC that meets defined risk significance criteria. In the LMP framework, an SSC } \\
\text { is regarded as risk-significant if its PRA Safety Function is: a) required to keep one } \\
\text { or more LBEs inside the F-C Target based on mean frequencies and consequences; } \\
\text { or b) if the total frequency LBEs that involve failure of the SSC PRA Safety Function } \\
\text { contributes at least } 1 \% \text { to any of the LMP cumulative risk targets. The LMP } \\
\text { cumulative risk targets include: (i) maintaining the frequency of exceeding } 100\end{array}$ & LMP \\
\hline
\end{tabular}


Modernization of Technical Requirements

\begin{tabular}{|c|c|c|c|}
\hline LMP Term & Acronym & Definition & Source \\
\hline & & $\begin{array}{l}\text { mrem to less than } 1 / \text { plant-year; (ii) meeting the NRC safety goal QHO for individual } \\
\text { risk of early fatality; and (iii) meeting the NRC safety goal QHO for individual risk of } \\
\text { latent cancer fatality. }\end{array}$ & \\
\hline $\begin{array}{l}\text { Safety-Significant } \\
\text { SSC }\end{array}$ & -- & $\begin{array}{l}\text { An SSC that performs a function whose performance is necessary to achieve } \\
\text { adequate defense-in-depth or is classified as risk-significant (see Risk-Significant } \\
\text { SSC.) }\end{array}$ & LMP \\
\hline $\begin{array}{l}\text { Safety Design } \\
\text { Approach }\end{array}$ & -- & $\begin{array}{l}\text { The strategies that are implemented in the design of a nuclear power plant that are } \\
\text { intended to support safe operation of the plant and control the risks associated } \\
\text { with accidental releases of radioactive material and protection of the public and } \\
\text { plant workers. These strategies normally include the use of robust barriers, } \\
\text { multiple layers of defense, redundancy, and diversity, and the use of inherent and } \\
\text { passive design features to perform safety functions. }\end{array}$ & LMP \\
\hline \multicolumn{4}{|c|}{ Terms Associated with Risk-Informed and Performance-Based Regulation and Decision-Making } \\
\hline Defense-in-Depth & DID & $\begin{array}{l}\text { "An approach to designing and operating nuclear facilities that prevents and } \\
\text { mitigates accidents that release radiation or hazardous materials. The key is } \\
\text { creating multiple independent and redundant layers of defense to compensate for } \\
\text { potential human and mechanical failures so that no single layer, no matter how } \\
\text { robust, is exclusively relied upon. Defense-in-depth includes the use of access } \\
\text { controls, physical barriers, redundant and diverse key safety functions, and } \\
\text { emergency response measures." }\end{array}$ & NRC Glossary \\
\hline Layers of Defense & -- & $\begin{array}{l}\text { Layers of defense are those plant capabilities and programmatic elements that } \\
\text { provide, collectively, independent means for the prevention and mitigation of } \\
\text { adverse events. The actual layers and number are dependent on the actual source } \\
\text { and hazard posing the threat. See Defense-in-Depth. }\end{array}$ & LMP \\
\hline $\begin{array}{l}\text { Performance- } \\
\text { Based }\end{array}$ & PB & $\begin{array}{l}\text { An approach to decision-making that focuses on desired objective, calculable or } \\
\text { measurable, observable outcomes, rather than prescriptive processes, techniques, } \\
\text { or procedures. Performance-based decisions lead to defined results without } \\
\text { specific direction regarding how those results are to be obtained. At the NRC, } \\
\text { performance-based regulatory actions focus on identifying performance measures } \\
\text { that ensure an adequate safety margin and offer incentives and flexibility for } \\
\text { licensees to improve safety without formal regulatory intervention by the agency. }\end{array}$ & $\begin{array}{l}\text { Adapted from NRC } \\
\text { Glossary definition of } \\
\text { performance-based } \\
\text { regulation in order to } \\
\text { apply to both design } \\
\text { decisions and regulatory } \\
\text { decision-making }\end{array}$ \\
\hline
\end{tabular}


Modernization of Technical Requirements

\begin{tabular}{|c|c|c|c|}
\hline LMP Term & Acronym & Definition & Source \\
\hline Risk-Informed & $\mathrm{RI}$ & $\begin{array}{l}\text { An approach to decision-making in which insights from probabilistic risk } \\
\text { assessments are considered with other sources of insights }\end{array}$ & $\begin{array}{l}\text { Adapted from NRC } \\
\text { Glossary definition of } \\
\text { performance-based } \\
\text { regulation in order to } \\
\text { apply to both design } \\
\text { decisions and regulatory } \\
\text { decision-making }\end{array}$ \\
\hline $\begin{array}{l}\text { Risk-Informed } \\
\text { and } \\
\text { Performance- } \\
\text { Based Integrated } \\
\text { Decision-Making }\end{array}$ & RIPB-DM & $\begin{array}{l}\text { The union of risk information and performance information to achieve } \\
\text { performance-based objectives }\end{array}$ & \\
\hline \multicolumn{4}{|c|}{ Terms Associated with Probabilistic Risk Assessment } \\
\hline Initiating Event & IE & $\begin{array}{l}\text { A perturbation to the plant during a plant operating state (POS) that challenges } \\
\text { plant control and safety systems whose failure could potentially lead to an } \\
\text { undesirable end state and/or radioactive material release. An Initiating Event could } \\
\text { degrade the reliability of a normally operating system, cause a standby mitigating } \\
\text { system to be challenged, or require that the plant operators respond in order to } \\
\text { mitigate the event or to limit the extent of plant damage caused by the IE. These } \\
\text { events include human-caused perturbations and failure of equipment from either } \\
\text { internal plant causes (such as hardware faults, floods, or fires) or external plant } \\
\text { causes (such as earthquakes or high winds). An IE is defined in terms of the change } \\
\text { in plant status that results in a condition requiring shutdown or a reactor trip (e.g., } \\
\text { loss of main feedwater system, small reactor coolant pressure boundary [RCPB] } \\
\text { breach) when the plant is at power, or the loss of a key safety function (e.g., decay } \\
\text { heat removal system) for non-power modes of operation. A specific type of IE may } \\
\text { be identified as originating from a specific cause as defined in terms such as "flood- } \\
\text { induced transient" or "seismically-induced RCPB breach." }\end{array}$ & $\begin{array}{l}\text { ASME/ANS-Ra-S-1.4- } \\
2013\end{array}$ \\
\hline Event Sequence & ES & $\begin{array}{l}\text { A representation of a scenario in terms of an Initiating Event defined for a set of } \\
\text { initial plant conditions (characterized by a specified POS) followed by a sequence of } \\
\text { system, safety function, and operator failures or successes, with sequence }\end{array}$ & $\begin{array}{l}\text { ASME/ANS-Ra-S-1.4- } \\
2013\end{array}$ \\
\hline
\end{tabular}




\begin{tabular}{|c|c|c|c|}
\hline LMP Term & Acronym & Definition & Source \\
\hline & & $\begin{array}{l}\text { termination with a specified end state (e.g., prevention of release of radioactive } \\
\text { material or release in one of the reactor-specific release categories. An event } \\
\text { sequence may contain many unique variations of events (minimal cut sets) that are } \\
\text { similar in terms of how they impact the performance of safety functions along the } \\
\text { event sequence. }\end{array}$ & \\
\hline $\begin{array}{l}\text { Event Sequence } \\
\text { Family }\end{array}$ & - & $\begin{array}{l}\text { A grouping of event sequences with a common or similar POS, IE, hazard group, } \\
\text { challenges to the plant safety functions, response of the plant in the performance of } \\
\text { each safety function, response of each radionuclide transport barrier, and end } \\
\text { state. An event sequence family may involve a single event sequence or several } \\
\text { event sequences grouped together. Each release category may include one or more } \\
\text { event sequence families. Event sequence families are not required to be explicitly } \\
\text { modeled in a PRA. Each event sequence family involving a release is associated } \\
\text { with one and only one release category. }\end{array}$ & \\
\hline End State & & $\begin{array}{l}\text { The set of conditions at the end of an Event Sequence that characterizes the impact } \\
\text { of the sequence on the plant or the environment. In most PRAs, end states typically } \\
\text { include success states (i.e., those states with negligible impact) and Release } \\
\text { Categories. }\end{array}$ & $\begin{array}{l}\text { ASME/ANS-Ra-S-1.4- } \\
2013\end{array}$ \\
\hline $\begin{array}{l}\text { PRA Technical } \\
\text { Adequacy }\end{array}$ & -- & $\begin{array}{l}\text { A set of attributes that define the technical suitability of a PRA capability to provide } \\
\text { fit-for-purpose insights to risk-informed decision-making. It includes consideration } \\
\text { of realism, completeness, transparency, PRA model-to-plant as-designed and as- } \\
\text { built fidelity state, and identification and evaluation of uncertainties relative to risk } \\
\text { levels. Strategies to achieve technical adequacy include conformance to consensus } \\
\text { PRA standards, performance of PRA peer reviews, and structured processes for PRA } \\
\text { model configuration control, maintenance and updates, and incorporation of new } \\
\text { evidence that comprises the state of knowledge reflected in the PRA model } \\
\text { development and its quantification. }\end{array}$ & LMP \\
\hline $\begin{array}{l}\text { Plant Operating } \\
\text { State }\end{array}$ & POS & $\begin{array}{l}\text { A standard arrangement of the plant during which the plant conditions are } \\
\text { relatively constant, are modeled as constant, and are distinct from other } \\
\text { configurations in ways that impact risk. POS is a basic modeling device used for a } \\
\text { phased-mission risk assessment that discretizes the plant conditions for specific } \\
\text { phases of an LPSD evolution. Examples of such plant conditions include core decay }\end{array}$ & $\begin{array}{l}\text { ASME/ANS-Ra-S-1.4- } \\
2013\end{array}$ \\
\hline
\end{tabular}


Modernization of Technical Requirements for Licensing of Advanced Non-Light Water Reactors: Selection and Evaluation of Licensing Basis Events

\begin{tabular}{|lll}
\hline LMP Term & Acronym & \multicolumn{1}{c}{ Definition } \\
& $\begin{array}{l}\text { heat level, primary coolant level, primary temperature, primary vent status, reactor } \\
\text { building status, and decay heat removal mechanisms. Examples of risk impacts that } \\
\text { are dependent on POS definition include the selection of IEs, IE frequencies, } \\
\text { definition of accident sequences, success criteria, and accident sequence } \\
\text { quantification. }\end{array}$ \\
\hline $\begin{array}{l}\text { Mechanistic } \\
\text { Source Term }\end{array}$ & $\begin{array}{l}\text { A source term that is calculated using models and supporting scientific data that } \\
\text { simulate the physical and chemical processes that describe the radionuclide } \\
\text { inventories and the time-dependent radionuclide transport mechanisms that are } \\
\text { necessary and sufficient to predict the source term. }\end{array}$ & ASME/ANS-Ra-S-1.4- \\
\hline
\end{tabular}




\subsection{REFERENCES}

[1] U.S. Nuclear Regulatory Commission, "NRC Staff High Level Comments Selection of Licensing Basis Events Draft Report Revision 0," ADAMS Accession No. ML17145A573, May 25, 2017.

[2] Nuclear Energy Institute, "Risk-Informed Performance-Based Technology Inclusive Guidance for Advanced Reactor Licensing Basis Development," NEI 18-04, Revision OB, May 25, 2019.

[3] Idaho National Laboratory, "Next Generation Nuclear Plant Licensing Basis Event Selection White Paper," INL/EXT-10-19521, ADAMS Accession No. ML102630246, September 2010.

[4] 10 CFR 52.1, "Licenses, Certifications, and Approvals for Nuclear Power Plants - Definitions," 2015.

[5] Idaho National Laboratory, "Modernization of Technical Requirements for Licensing of Advanced Non-Light Water Reactors: Probabilistic Risk Assessment Approach," Revision 0, August 2019.

[6] Idaho National Laboratory, "Modernization of Technical Requirements for Licensing of Advanced Non-Light Water Reactors: Safety Classification and Performance Criteria for Structures, Systems and Components," Revision 0, August 2019.

[7] Idaho National Laboratory, "Modernization of Technical Requirements for Licensing of Advanced Non-Light Water Reactors: Risk-Informed and Performance-Based Evaluation of Defense-in-Depth Adequacy," Revision 0, August 2019.

[8] NUREG-0800, Standard Review Plan, Chapter 15.0, "Introduction - Transient and Accident Analyses," Revision 3, March 2007.

[9] 10 CFR 50, Appendix A, "General Design Criteria for Nuclear Power Plants," Code of Federal Regulations, Office of the Federal Register, 2007.

[10] 10 CFR 50.62, "Requirements for reduction of risk from anticipated transients without scram (ATWS) events for light-water-cooled nuclear power plants," 2015.

[11] 10 CFR 50.63, "Loss of all alternating current power," 2015.

[12] 10 CFR 50.44, "Combustible gas control for nuclear power reactors," 2015.

[13] 10 CFR 50.150, "Aircraft impact assessment," 2015.

[14] U.S. Nuclear Regulatory Commission, "Policy Statement on the Regulation of Advanced Reactors," 73 FR 60612, October 14, 2008.

[15] U.S. Nuclear Regulatory Commission, "Policy Statement on Use of Probabilistic Risk Assessment Methods in Nuclear Regulatory Activities," 60 FR 42622, August 16, 1995.

[16] U.S. Nuclear Regulatory Commission, "Policy Statement on Safety Goals for Operation of Nuclear Power Plants," 51 FR 28044, August 21, 1986.

[17] U.S. Nuclear Regulatory Commission, "Policy Statement on Severe Reactor Accidents Regarding Future Designs and Existing Plants," 50 FR 32138, August 8, 1985.

[18] SECY 2002-0076, "Semi-Annual Update of the Future Licensing and Inspection Readiness Assessment (FLIRA)," May 8, 2002.

[19] SECY 2003-0047, "Policy Issues Related to Licensing Non-Light Water Reactor Designs," U.S. Nuclear Regulatory Commission, March 28, 2003.

[20] SRM 2003-0047, "Staff Requirements Memorandum for SECY 03-0047-Policy Issues Related to Licensing Non-Light Water Reactor Designs," U.S. Nuclear Regulatory Commission, June 26, 2003. 
[21] SECY-2005-0006, "Second Status Paper on the Staff's Proposed Regulatory Structure for New Plant Licensing and Update on Policy Issues Related to New Plant Licensing," January 7, 2005.

[22] SECY-2010-0034, "Potential Policy, Licensing, and Key Technical Issues for Small Modular Nuclear Reactor Designs," March 28, 2010.

[23] SECY-2011-0079, "License Structure for Multi-Module Facilities Related to Small Modular Nuclear Power Reactors," June 12, 2011.

[24] NUREG-0800, Standard Review Plan, Chapter 19.0, "Probabilistic Risk Assessment and Severe Accident Evaluation for New Reactors," Revision 3 December 2015.

[25] SECY 2016-0012, "Accident Source Terms and Siting for Small Modular Reactors and Non-Light Water Reactors," February 7, 2016.

[26] NUREG-1860, "Feasibility Study for a Risk-Informed and Performance-Based Regulatory Structure for Future Plant Licensing," U.S. Nuclear Regulatory Commission, December 2007.

[27] NUREG-2150, “A Proposed Risk Management Regulatory Framework," U.S. Nuclear Regulatory Commission, April 2012.

[28] U.S. Nuclear Regulatory Commission, "The Near-Term Task Force Review of Insights from the Fukushima Dai-Ichi Accident," July 12, 2011.

[29] NUREG/BR-0303, “Guidance for Performance Based Regulation," U.S. Nuclear Regulatory Commission, December 2002.

[30] Idaho National Laboratory, "Next Generation Nuclear Plant Defense-in-Depth Approach White Paper," INL/EXT-09-17139, ADAMS Accession No. ML102630246, March 2009.

[31] Department of Energy, "Yucca Mountain Repository Safety Analysis Report" Chapters 1.6 - 1.9 and 1.14, Docket No. 63-001, DOE/RW-0573, Rev. 1, February 2009.

[32] NUREG-2108, "Technical Evaluation Report on the Content of the U.S. Department of Energy's Yucca Mountain Repository License Application: Preclosure Volume: Repository Safety Before Permanent Closure," Nuclear Regulatory Commission, Office of Nuclear Material Safety and Safeguards, September 2011.

[33] 10 CFR 63, "Disposal of High-Level Radioactive Wastes in a Geologic Repository at Yucca Mountain, Nevada," 2016.

[34] NUREG-1804, "Yucca Mountain Review Plan," Revision 2, July 2003.

[35] ASME/ANS RA-Sb-2013, "Addenda to ASME/ANS RA-S-2008 Standard for Level 1/Large Early Release Frequency Probabilistic Risk Assessment for Nuclear Power Plant Applications," American Society of Mechanical Engineers and American Nuclear Society, September 2013.

[36] ASME/ANS RA S-1.4-2013, "Probabilistic Risk Assessment Standard for Advanced Non-LWR Nuclear Power Plants," American Society of Mechanical Engineers and American Nuclear Society, December 2013.

[37] 10 CFR 63.111, "Performance objectives for the geologic repository operations area through permanent closure," 2015.

[38] 10 CFR 63.112, "Requirements for preclosure safety analysis of the geologic repository operations area," 2015. 
[39] SRM 90-16, "Staff Requirements Memorandum for SECY 90-16 Evolutionary Light Water Reactor Certification Issues and Their Relationship to Current Regulatory Requirements," January 1990.

[40] Regulatory Guide 1.174, "An Approach for Using Probabilistic Risk Assessment in Risk-informed Decisions on Plant Specific Changes to the Current Licensing Basis," Federal Register, U.S. Nuclear Regulatory Commission, Revision 2, May 2011.

[41] Regulatory Guide 1.200, "An Approach for Determining the Technical Adequacy of Probabilistic Risk Assessment Results for Risk-informed Activities," Federal Register, U.S. Nuclear Regulatory Commission, Revision 2, March 2009.

[42] International Atomic Energy Agency, "Safety Standards Series: Safety of Nuclear Power Plants Design Requirements," NSR-1, September 2000.

[43] United Kingdom Office of Nuclear Regulation, "Safety Assessment Principles for Nuclear Facilities," Revision 0, 2014 Edition.

[44] U.S. Department of Energy, "Preliminary Safety Information Document for the Standard MHTGR," DOE-HTGR-86-024, September 1988.

[45] U.S. Department of Energy, "Probabilistic Risk Assessment for the Standard Modular High Temperature Gas-Cooled Reactor," DOE-HTGR-86-011, Revision 5, April 1988.

[46] NUREG-1338, "Draft Preapplication Safety Evaluation Report for the Modular High Temperature Gas-Cooled Reactor," U.S. Nuclear Regulatory Commission, March 1989.

[47] NUREG-1368, "Preapplication Safety Evaluation Report for the Power Reactor Innovative Small Module (PRISM) Liquid-Metal Reactor," Final Report, U.S. Nuclear Regulatory Commission, February 1994.

[48] Edward G. Wallace letter to NRC Document Control Desk, "PBMR White Paper: LBE Selection," July 3, 2006.

[49] NRC letter to PBMR, "Requests for Additional Information Regarding Pebble Bed Modular Reactor (PBMR) Pre-Application White Papers," September 24, 2007.

[50] PBMR Limited letter to NRC, "Response to Requests for Additional Information," March 21, 2008.

[51] Exelon Generation Company Letter, Subject: Proposed Licensing Approach for the Pebble Bed Modular Reactor in the United States, January 31, 2002.

[52] U.S. Nuclear Regulatory Commission Letter, Subject: "NRC Staff's Preliminary Findings Regarding Exelon Generation's (Exelon's) Proposed Licensing Approach for The Pebble Bed Modular Reactor (PBMR)," March 26, 2002. See also SECY-95-0299, "Licensing Approach for Exelon's Pebble Bed Modular Reactor," December 19, 1995.

[53] INL/EXT-13-28205, "NRC Licensing Status Summary Report for NGNP", Idaho National Laboratory, November 2014.

[54] Letter from J. Sam Armijo, Chairman U.S. Nuclear Regulatory Commission Advisory Committee on Reactor Safeguards to Mr. R.W. Borchardt, Executive Director for Operations, U.S. Nuclear Regulatory Commission Letter, "Next Generation Nuclear Plant (NGNP) Key Licensing Issues," May 15, 2013.

[55] SECY 2011-0152, "Development of an Emergency Planning and Preparedness Framework for Small Modular Reactors," October 28, 2011. 
[56] 10 CFR 20, "Standards for Protection against Radiation Subpart C, Occupational Dose Limits," 2016.

[57] 10 CFR 20, "Standards for Protection against Radiation Subpart D, Radiation Dose Limits for Individual Members of the Public," 2016.

[58] 10 CFR 50, Appendix I, “Numerical Guides for Design Objectives and Limiting Conditions for Operation to Meet the Criterion 'As Low as is Reasonably Achievable' for Radioactive Material in Light-Water-Cooled Nuclear Power Reactor Effluents," 2015.

[59] 10 CFR 52, Licenses, Certifications, and Approvals for Nuclear Power Plants, Subpart C "Combined Licenses," 2016.

[60] 10 CFR 52.79, "Contents of applications; technical information in final safety analysis report," 2015.

[61] 40 CFR 190, "Environmental Radiation Protection Standards for Nuclear Power Operations," 2016.

[62] 10 CFR 100, "Reactor Site Criteria (Subpart B, Evaluation Factors for Stationary Power Reactor Site Applications on or After January 10, 1997)."

[63] 10 CFR 50.34, "Contents of Applications: Technical Information - Radiological Dose Consequences," 2015.

[64] 10 CFR 50.34a, "Design objectives for equipment to control releases of radioactive material in effluents-nuclear power reactors," 2015.

[65] 40 CFR 190.10, "Standards for normal operations," 2016.

[66] Idaho National Laboratory, "Next Generation Nuclear Plant Probabilistic Risk Assessment White Paper," INL/EXT-11-21270, Idaho National Laboratory, September 2011.

[67] NUREG-0800, Standard Review Plan, Chapter 15.0.3 "Design Basis Accident Radiological Consequence Analyses for Advanced Light Water Reactors," March 2007.

[68] Farmer, F.R., "Reactor Safety and Siting: A Proposed Risk Criterion," Nuclear Safety, pp. 539-48, 1967.

[69] American Nuclear Society, ANSI/ANS-53.1-2011, "Nuclear Safety Design Process for Modular Helium-Cooled Reactor Plants," December 21, 2011.

[70] NEDO-33201, Revision 6, ESBWR Design Certification Probabilistic Risk Assessment, Chapter 10.4 Comparison of Results to Goals, Adams Accession No. ML102880536.

[71] U.S. Department of Energy, "Licensing Basis Events for the Standard MHTGR," DOE-HTGR-86-034, February 1987.

[72] GE Hitachi Nuclear Energy, "Final Scientific/Technical Report: Development/Modernization of an Advanced Non-LWR Probabilistic Risk Assessment," Federal Grant DE-NE0008325, 2017.

[73] Letter from Mario V. Bonaca, Chairman U.S. Nuclear Regulatory Commission Advisory Committee on Reactor Safeguards to Honorable Nils Diaz, Chairman, U.S. Nuclear Regulatory Commission Letter, "Options and Recommendations for Policy Issues Related to Licensing Non-Light Water Reactor Designs," April 22, 2004.

[74] SECY 2013-0029, "History of the Use and Consideration of the Large Release Frequency Metric by the U.S. Nuclear Regulatory Commission," March 22, 2013. 
[75] University of California, Berkeley, "Fluoride-Salt-Cooled, High-Temperature Reactor (FHR) Subsystems Definition, Functional Requirement Definition, and Licensing Basis Event (LBE) Identification White Paper," UCBTH-12-001, August 2013.

[76] ORNL/TM-2018/788, "A New Look at Licensing Basis Events for the Molten Salt Reactor Experiment," August 2018.

[77] Idaho National Laboratory, "Modernization of Technical Requirements for Licensing of Advanced Non-Light Water Reactors, High Temperature, Gas-Cooled Pebble Bed Reactor Licensing Modernization Project Demonstration," Revision 0, August 2018.

[78] Idaho National Laboratory, "Modernization of Technical Requirements for Licensing of Advanced Non-Light Water Reactors, PRISM Sodium Fast Reactor Licensing Modernization Project Demonstration," Revision 0, December 2018.

[79] Vanderbilt University, "Molten Salt Reactor Experiment (MSRE) Case Study Using Risk-Informed, Performance-Based Technical Guidance to Inform Future Licensing for Advanced Non-Light Water Reactors", September 4, 2019

[80] Idaho National Laboratory, "Modernization of Technical Requirements for Licensing of Advanced Non-Light Water Reactors, Flouride-Cooled High Temperature Reactor Licensing Modernization Project Demonstration," Revision 0, September 2019.

[81] Idaho National Laboratory, "Modernization of Technical Requirements for Licensing of Advanced Non-Light Water Reactors, Westinghouse eVinci ${ }^{\mathrm{TM}}$ Micro-Reactor Licensing Modernization Project Demonstration," Revision 0, August 2019.

[82] 
APPENDIX A. REGULATORY FOUNDATION AND PRECEDENTS 


\section{Table of Contents}

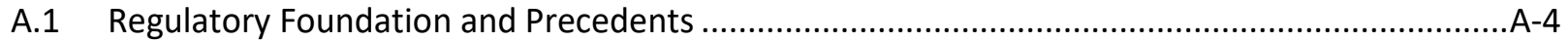

A.1.1 U.S. Regulatory Foundation for the Selection of LBEs ............................................A-4

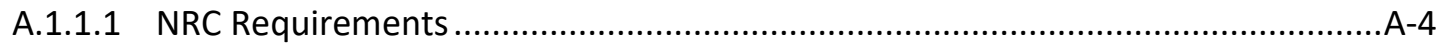

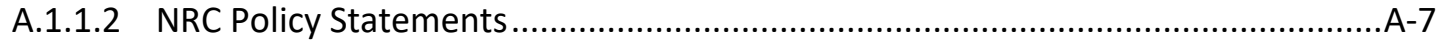

A.1.1.2.1 Advanced Reactor Policies................................................................ A-7

A.1.1.2.2 NRC Small Modular Reactor Precedents ............................................. A-10

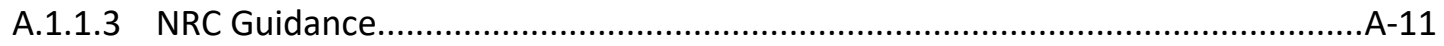

A.1.1.3.1 Standard Review Plan (NUREG-0800)................................................. A-11

A.1.1.3.2 NUREG-1860 Feasibility Study for a Risk-Informed and PerformanceBased Regulatory Structure for Future Plant Licensing ${ }^{[21]}$...................... A-13

A.1.1.3.3 NUREG-2150 A Proposed Risk Management Regulatory Framework ${ }^{[22]}$. A-16

A.1.1.3.4 Fukushima Accident and NRC Near-Term Task Force (NTTF) Report ${ }^{[23]}$.. A-17

A.1.1.3.5 NRC Guidance for Performance-Based Regulation ${ }^{[24]}$.......................... A-19

A.1.2 International Guidance for Licensing Basis Event Selection...........................................20

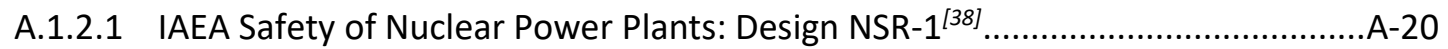

A.1.2.2 United Kingdom Safety Assessment Principles for Nuclear Facilities ${ }^{[39]}$..............A-20

A.1.3 Historical Precedents for Advanced non-LWRs .........................................................

A.1.3.1 Modular High-Temperature Gas-Cooled Reactor Pre-Application Review ..........A-21

A.1.3.2 Exelon PBMR Pre-Application Review...............................................................22

A.1.3.3 Next Generation Nuclear Plant (NGNP) Licensing Approach Review ..................A-24

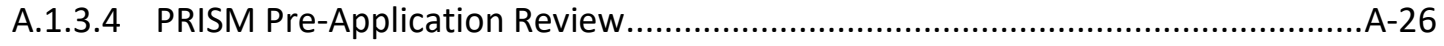

A.1.3.5 ANS Design Standard for Modular Helium Cooled Reactor Plants ${ }^{[25]}$.................... 28

A.1.3.6 Yucca Mountain Pre-closure Safety Analysis (PCSA) ${ }^{[27]}$........................................30

A.1.4 Regulatory Foundation for Establishing RIPB Frequency-consequence targets .............A-34

A.1.4.1 F-C Targets Related to Normal Operation and AOOs...........................................36

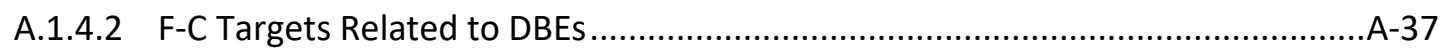

A.1.4.3 F-C Targets Related to Policy Guidance for BDBEs..........................................A-40

A.1.4.4 Criteria for Classifying LBEs Based on Frequency of Occurrence ........................A-41

A.1.4.5 United Kingdom Safety Assessment Principles Numerical Targets .....................A-42

A.1.5 Regulatory Foundation Precedent Review Summary …............................................ 44

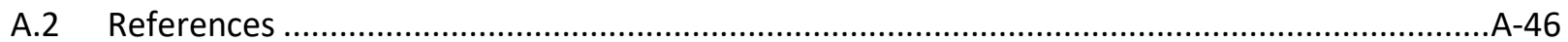




\section{List of Figures and Tables}

Figure A-1. Regulatory Framework Proposed in NUREG-2150 …................................................... A-16

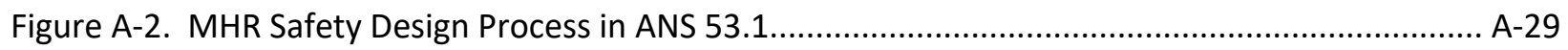

Figure A-3. Use of the PCSA for Risk Management of Repository Design ......................................... A-33

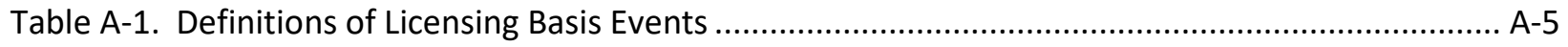

Table A-2. NRC Risk Management Task Force Recommendations for Generation IV Reactors ............ A-17

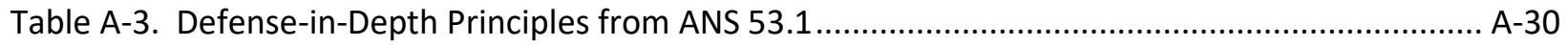

Table A-4. Performance Criteria for Category 1 and 2 Event Sequences and Normal Operation ......... A-32

Table A-5. Accident-Dose Criteria (Table 1 from Reference [63]) ….................................................. A-39

Table A-6. United Kingdom Safety Assessment Principles Numerical Risk Targets ${ }^{[39]}$......................... A-43 


\section{A.1 REGULATORY FOUNDATION AND PRECEDENTS}

There is a substantial set of prior activities, policies, practices, and precedents stretching more than 30 years back in time that inform RIPB processes and uses. NRC and international regulations, policies, guidance, and other precedents that are relevant to the definition of LBEs and their treatment are discussed in this appendix. NRC and ACRS feedback on previous efforts to define LBEs for Advanced Non-LWRs are also reviewed for LBE definition guidance. This regulatory background is examined to investigate two aspects of the proposed TI-RIPB approach for the LMP project. The first is the process of defining and selecting the LBEs and the second is the selection of the Top-Level Safety Targets* (TLSTs) that are used to establish evaluation boundaries on the frequencies and radiological consequences for classifying and evaluating the LBEs. These boundaries are referred to in the LMP methodology as Frequency-Consequence (F-C) Targets. The scope of this review includes U.S. regulatory requirements as specified in the regulations, and supporting policies, NRC directives, regulatory guidance, and Standard Review Plan, as well as international safety standards. Insights from NRC pre-licensing reviews of advanced non-LWRs are also included. This section of the report builds on the regulatory review in the NGNP White Paper on LBE selection ${ }^{[1]}$ by incorporating more recent developments and precedents and by considering the need to have a reactor technology-inclusive approach for selecting LBEs rather than one focused on HTGR-specific technology only. Observations and conclusions reached from this review that are used in the definition of the LBE approach are summarized at the end of this section.

\section{A.1.1 U.S. REGULATORY FOUNDATION FOR THE SELECTION OF LBES}

\section{A.1.1.1 NRC REQUIREMENTS}

This section reviews NRC requirements for insights on how to select LBEs for a new reactor design. This discussion reflects on the qualitative approach to risk used in the past, relying on judgment and prescription derived from years of LWR design, analysis, and operations. The purpose is not to criticize, but rather to identify desirable attributes of a TI-RIPB approach to the selection of LBEs.

$\mathrm{NRC}$ regulatory requirements for the design of currently licensed and new reactors refer to several different kinds of events included within the licensing basis including Anticipated Operational Occurrences (AOOs), Design Basis Events (DBEs), postulated accidents, Design Basis Accidents (DBAs), and Beyond Design Basis Events (BDBEs). The definitions of these events are similar to LBE types introduced in Section 2.4. However, as shown in Table A-1, there are significant differences in licensing event terminology.

\footnotetext{
* The LMP term "Top Level Safety Targets" was referred to in the Next Generation Nuclear Plant (NGNP) project as "Top Level Regulatory Criteria (TLRC)." The LMP changed the term to TLST emphasize that the frequency-consequence criteria derived from these inputs are to be used as design targets and are not considered regulatory limits.
} 


\section{Table A-1. Definitions of Licensing Basis Events}

\section{Event Type}

Anticipated

Operational

Occurrences

(AOOs)

power." [SRP 15.0 and 10 CFR 50 Appendix A]

Events external events, and natural phenomena, for which the plant must be

(DBEs) designed to ensure functions of safety-related electric equipment that ensures the integrity of the reactor coolant pressure boundary; the capability to shut down the reactor and maintain it in a safe shutdown condition; or the capability to prevent or mitigate the consequences of accidents that could result in potential offsite exposures." [SRP 15.0]

\section{Beyond "This term is used as a technical way to discuss accident sequences that} Design Basis are possible but were not fully considered in the design process because Events they were judged to be too unlikely. (In that sense, they are considered

(BDBEs) beyond the scope of design-basis accidents that a nuclear facility must be designed and built to withstand.) As the regulatory process strives to be as thorough as possible, 'beyond design-basis' accident sequences are analyzed to fully understand the capability of a design." [NRC Glossary]

Design Basis "Postulated accidents that are used to set design criteria and limits for Accidents the design and sizing of safety-related systems and components." (DBAs) [SRP 15.0]

"A postulated accident that a nuclear facility must be designed and built to withstand without loss to the systems, structures, and components necessary to ensure public health and safety." [NRC Glossary and NUREG-2122]

Licensing This term is not used formally in NRC documents.

Basis Events

(LBEs)

\section{LMP Definition}

Anticipated event sequences expected to occur one or more times during the life of a nuclear power plant, which may include one or more reactor modules. Event sequences with mean frequencies of $1 \times 10^{-2} /$ plant-year and greater are classified as AOOs. AOOs take into account the expected response of all SSCs within the plant, regardless of safety classification.

Infrequent event sequences that are not expected to occur in the life of a nuclear power plant, which may include one or more reactor modules, but are less likely than AOOs. Event sequences with mean frequencies of $1 \times 10^{-4} /$ plant-year to $1 \times 10^{-2} /$ plant-year are classified as DBEs. DBEs take into account the expected response of all SSCs within the plant regardless of safety classification.

Rare event sequences that are not expected to occur in the life of a nuclear power plant, which may include one or more reactor modules, but are less likely than a DBE. Event sequences with mean frequencies of $5 \times 10^{-7} /$ plantyear to $1 \times 10^{-4} /$ plant-year are classified as BDBEs. BDBEs take into account the expected response of all SSCs within the plant regardless of safety classification.

Postulated event sequences that are used to set design criteria and performance objectives for the design of SR SSCs. DBAs are derived from DBEs based on the capabilities and reliabilities of SR SSCs needed to mitigate and prevent event sequences, respectively. DBAs are derived from the DBEs by prescriptively assuming that only SR SSCS are available to mitigate postulated event sequence consequences to within the 10 CFR 50.34 dose limits.

The entire collection of events considered in the design and licensing basis of the plant, which may include one or more reactor modules. LBEs include AOOs, DBEs, BDBEs, and DBAs. * SRP 15.0 further breaks down AOOs into events with "moderate" frequency (events expected to occur several times during the plant life) and "infrequent" (events that may occur
during the plant life). 
For normal operations, including AOOs, the NRC regulations are, for the most part, generic and appear to generally apply to an advanced non-LWR plant. The applicant is required to classify the events considered within the design basis as either AOO or accident (DBA) based on a qualitative and presumably subjective assessment of the expected frequency of occurrence because there are no quantitative frequency criteria included. In many cases it is unclear whether the qualitative characterization of frequency refers to that for an initiating event or for an entire accident sequence. While the applicant's classification is subjected to NRC staff review, there is no quantification of the event frequencies nor a prescribed method for ensuring that design specific events are adequately considered. A concern for advanced non-LWRs is that events that are uniquely appropriate for a given reactor technology are likely not represented on the supplied lists of generic LWR events, so it is necessary to have a method that is systematic and reproducible to derive the appropriate list of LBEs. For non-LWR plants whose designs depart in major ways from those of existing and even advanced LWRs, a more systematic and quantitative means of identifying the unique events and correctly classifying their frequencies would be necessary to ensure a safe design and contribute to a more predicable path to a license. Moreover, establishing an appropriate set of reactor technology-specific LBEs cannot wait until the submittal of a license application. This selection is essential to the development of any design and must be established very early in the design process.

All the example events given in the definition of AOO in the regulations and in the supporting regulatory guides and Standard Review Plan ${ }^{[3]}$ are applicable to LWRs. Many of these may not be applicable to a particular non-LWR design.

In the selection of LBEs, it is expected that the selection will consider a comprehensive and exhaustive set of events from which to identify the "limiting" events. However, specific criteria for how to determine which events are limiting are not provided in existing regulatory guidance. In addition, it is not clear from the regulatory guidance which events are considered to be limited by the selected events. This points to a need for a systematic and reproducible process to identify the DBAs for the deterministic safety analysis.

With few exceptions, such as provisions for protection against natural phenomena and inclusion of some generic events in the lists of example events such as loss of offsite power and station blackout, the regulations that have evolved for unplanned transients and accidents are LWR-specific. The GDC define the types of design considerations that apply to the design of SSCs that prevent or mitigate a specified set of postulated accidents. For example, GDC typically indicate that safety systems must be able to perform their design basis functions given a single active failure and a concurrent loss of offsite power.

NRC regulations do not have performance-based criteria to limit the consequences of BDBEs nor quantitative criteria for classifying events as BDBEs based on frequency other than noting they were considered too infrequent to be included in the design basis. In apparent response to events that have occurred but had not been anticipated in the original design and licensing bases, regulations have been added to provide protection against selected BDBEs. Examples of these include: anticipated transients without scram (ATWS) addressed in 10 CFR $50.62^{[5]}$ and station blackout (SBO) addressed in 10 CFR 50.63.

\footnotetext{
* One additional definition is required to understand the importance of the terms. The "design bases" means that information which identifies the specific functions to be performed by a structure, system, or component of a facility, and the specific values or ranges of values chosen for controlling parameters as reference bounds for design. These values may be (1) restraints derived from generally accepted state-of-the-art practices for achieving functional goals, or (2) requirements derived from analysis (based on calculation and/or experiments) of the effects of a postulated accident for which a structure, system, or component must meet its functional goals. [10 CFR50.2 Definitions]
} 
The regulations associated with licensing events and their supporting regulatory documents do not distinguish well between events and event sequences for the purpose of characterizing the frequency of occurrence and classifying as either an AOO, DBA, or BDBE. The term "sequence of events" is referred to here in the context of analyzing how the plant responds to initiating events. The point here is a given event may be characterized at a certain frequency level and severity of plant impact, but when compounded by additional failures both the frequency and the level of impact are different. Hence, there may be different LBEs having different levels of frequency and severity stemming from the same initiating event. In reviewing the regulatory documents, it is extremely difficult to sort out in most cases whether the term "events" refers to initiating events only or to some sequence of events. A goal of the LMP is to consider initiating events and the associated event sequences as distinct challenges to the safety functions in order to provide sufficient completeness in the identification of LBEs.

In many cases the events classified as AOOs or DBAs as discussed in the regulations and supporting SRP are referred to as "initiating events." By applying the single failure criterion, the safety analysis for the DBAs includes the requirement that the "worst" active single failure be assumed in demonstrating that safety criteria are met. However, the probability of the single failure does not have any bearing on the classification of the event. In addition, non-safety related SSCs including offsite power supplies are assumed not to be available in the deterministic safety analysis of DBAs which also would be considered if the frequency of the DBA were to be assessed.

With few exceptions, there does not appear to be a consideration of the probability of a common cause failure that could occur in combination with an initiating event to produce a DBA even though the service experience indicates that there have been many occurrences of such events. The application of the single failure criterion for DBAs seems to assume that common cause failures will be prevented by meeting the design requirements. In the limited cases of selected BDBE requirements such as those for ATWS and SBO, event sequences that could be caused in part by a common cause failure and involve multiple failures of redundant components are identified as being comprised of a sequence initiated by an AOO (transient for ATWS and loss of offsite power for SBO). However, a systematic way to consider both events and event sequences that could be comprised of combinations of single failures and common cause failures is not included in the enumeration of prescriptive events nor in the characterization of their frequencies or level of severity of the challenge.

An important insight from this review is that, based on what can be gleaned from the regulations and supporting documents, the historical approach to selecting an appropriate set of LBEs for a given design is ad hoc. The challenge facing designers and licensees for advanced non-LWRs is to find a process for selecting LBEs that is systematic, reproducible, and capable of identifying the appropriate limiting events for a given design.

\section{A.1.1.2 NRC POLICY STATEMENTS}

Advanced non-LWR designs need to adhere to relevant NRC policies. Each of the reviewed policies was examined for insights on the desirable attributes for an effective approach to select an appropriate set of LBEs for an advanced non-LWR.

\section{A.1.1.2.1 Advanced Reactor Policies}

Advanced non-LWR designs that may benefit from the LMP licensing strategy as expressed in this paper and associated documents are implementing design features identified in the NRC's Advanced Reactor Policy, which was revised in 2008. ${ }^{[9]}$ For advanced non-LWR reactor designs, the NRC expects at least the same degree of protection of the environment, public health and safety, and common defense and 
security that is required for current generation LWRs. The NRC also expects that advanced reactors will provide enhanced margins of safety and/or use simplified, inherent, passive, or other innovative means to accomplish their safety and security functions.

This Advanced Reactor Policy does not offer or refer to any guidance as to how LBEs will be defined for such reactors. It is reasonable to assume that reactors that rely more on passive and inherent safety features and less on active systems, use different materials for reactor fuel, coolant, and moderator, and have different design and configuration for radionuclide barriers may have limiting LBEs that are unique and specific relative to those previously defined for LWRs.

In 1995 the NRC issued its policy on the use of PRA methods in the regulatory process. ${ }^{[10]}$ The essence of this policy is reflected in the following statement:

"This statement reflects the policy that the Nuclear Regulatory Commission (NRC) will follow in the use of probabilistic risk assessment (PRA) methods in nuclear regulatory matters. The Commission believes that an overall policy on the use of PRA methods in nuclear regulatory activities should be established to that the many potential applications of PRA can be implemented in a consistent and predictable manner that would promote regulatory stability and efficiency. In addition, the Commission believes that the use of PRA technology in NRC regulatory activities should be increased to the extent supported by the state-of-the-art in PRA methods and data and in a manner that complements the NRC's deterministic approach...."

A key objective of the LBE selection approach described in this paper is to have an approach that is consistent with this policy statement which clearly articulates the need for a risk-informed approach.

Another key policy that is essential to help implement risk-informed decision making is the NRC Safety Goal Policy, ${ }^{[12]}$ whose purpose is summarized in the following statement:

"This policy statement focuses on the risks to the public from nuclear power plant operation. Its objective is to establish goals that broadly define an acceptable level of risk. In developing the policy statement, the NRC sponsored two public workshops during 1981, conducted a 2 year evaluation during 1983 to 1985, and received the views of its Advisory Committee on Reactor Safeguards."

This safety goal policy established two qualitative safety goals, supported by two quantitative health objectives bases on the principle that nuclear risks should be a small fraction of other societal and individual risks. This policy reflects the NRC's judgment on the question of "how safe is safe enough?" and provides goals and criteria which are used in Section 4 of this report to evaluate the risk significance of LBEs and the overall plant risks resulting from an entire collection of LBEs.

SECY 2002-0076, "Semi-Annual Update of the Future Licensing and Inspection Readiness Assessment," ${ }^{[13]}$ described Exelon's proposed licensing approach for the pebble bed HTGR design. Exelon proposed conformance with current regulations but recognized that many of the regulatory requirements were based on LWR technology. A risk-informed process would be employed to define plant design events, acceptance criteria, and SSCs. In its preliminary evaluation, NRC staff concluded that the proposed licensing approach, if adequately implemented, was a reasonable process for ensuring that the Commission's regulations would be met and for identifying pebble bed modular reactor (PBMR)specific regulatory requirements. 
SECY 2003-0047, "Policy Issues Related to Licensing Non-Light Water Reactor Designs"[14] offers staff recommendations on several relevant policy issues that had been originally defined in an earlier policy statement (SECY 2002-0139). Of these issues, Issue 4, "Use of PRA to Support Licensing Basis," specifically relates to the treatment of LBEs and is discussed herein. The Staff Requirements Memorandum (SRM) for SECY 2003-0047 ${ }^{[15]}$ stated the Commissioners approval of the staff recommendations on this issue.

With respect to Issue 4, the staff recommended that the Commission take the following actions:

"Modify the Commission's guidance, as described in the SRM of July 30, 1993, to put greater emphasis on the use of risk information by allowing the use of a probabilistic approach in the identification of events to be considered in the design, provided there is sufficient understanding of plant and fuel performance and deterministic engineering judgment is used to bound uncertainties.

Allow a probabilistic approach for the safety classification of structures, systems, and components.

Replace the single failure criterion with a probabilistic (reliability) criterion."

This recommendation is consistent with a risk-informed approach. It should be noted that this recommendation expands the use of PRA into forming part of the basis for licensing and thus puts greater emphasis on PRA quality, completeness, and documentation.

Also included, but left unresolved from the issues of SECY 2003-0047, were policy issues associated with the treatment of integrated risk on multi-module plants and for modular reactor designs, which are part of Issue 1 as stated in the SECY.

In its SRM to SECY-2003-047 the Commission asked the staff to identify options and provide more specifics on the treatment of integrated risks for multi-module plants. In response, the staff issued SECY 2005-0006, ${ }^{[16]}$ in which the staff recommended that the integrated risks of multi-module plants be addressed. In characterizing the risks from a multi-module plant, the staff noted that two different types of event sequences need to be considered:

"It should also be noted that in assessing the risk from plants consisting of multiple reactor modules, the event sequences that contribute to risk will generally fall into two basic categories (1) those that affect each reactor module individually and (2) those that can affect two or more modules simultaneously (e.g., seismic events). Accordingly, the overall risk from a plant comprised of multiple reactor modules consists of the sum of the risk from both categories, and may be lower than the sum of the risk from all modules if they were treated separately, particularly if some systems are shared among reactor modules. This would be due to the fact that the risk from event sequences that affect all reactor modules simultaneously may not be equal among the reactor modules."

In this SECY the staff recommended that advanced non-LWR plants with multi-module designs assess the integrated risk of the facility according to an Option 3 with consideration of both frequency and power level of the reactor modules. The other two options considered were an Option 1 in which integrated risk is not considered, and an Option 2 in which the integrated risk would be included by considering the 
frequency of both single and multiple reactor accidents but not addressing the power level in the assessment of consequences:

"On this basis, the staff has developed a proposed position endorsing Option 3. Option 3 realistically accounts for modular reactor characteristics by treating accident prevention independent of reactor power, while allowing the assessment of accident mitigation risk measures to consider reactor power, thus not imposing a de facto more stringent goal than implied by the Safety Goal Policy. In addition, Option 3 would be most consistent with the proposed Energy Bill language that would allow a set of reactor modules to be treated as a single unit for the purposes of financial protection (i.e., the risk from the set of reactor modules should not exceed that from a single large reactor). Option 3 would result in staff treatment of the risk associated with modular reactors as follows:

- taking into consideration the integrated effect of risk when assessing accident prevention for modular reactor designs, independent of reactor power level, and

- taking into consideration the integrated effect of risk when assessing accident mitigation for modular reactor designs in a fashion that allows for consideration of the effect of reactor power level."

In 2006 the Commission in its SRM to SECY 06-007 approved the NRC staff's recommendation to issue an Advanced Notice of Proposed Rulemaking (ANPR) on approaches for making technical requirements for power reactors risk-informed, performance-based, and technology neutral, subject to the comments and edits provided in the SRM. The staff recommendation identified a number of policy issues including the question of the level of safety to be required for advanced reactors and the question of the integrated risk of multiple module plants. The Commission approved the staff's recommendation to supplement the ANPR with new information, as needed. Subsequently, based on industry feedback that it was premature to propose rulemaking for advanced reactors, a revised recommendation was made in SECY-07-0101 to defer rulemaking until there was a license application for a PBMR or NGNP reactor. This recommendation was approved by the Commission in the SRM to SECY 07-0101, and hence policy issues for licensing advanced non-LWRs dating back to SECY 2003-0047 were never resolved.

The appreciation that accident sequences may involve two or more reactors has continued to evolve and was certainly manifested during the Fukushima Daiichi accident that produced core damage and containment breach on three reactor units.

\section{A.1.1.2.2 NRC Small Modular Reactor Precedents}

Because most advanced non-LWRs will employ a modular reactor design approach, a review of NRC policies for licensing SMRs will be beneficial in developing a suitable LBE approach.

SECY 2010-0034, "Potential Policy, Licensing, and Key Technical Issues for Small Modular Nuclear Reactor Designs," $[17]$ identifies a number of potential policy and licensing issues based on the preliminary design information provided by pre-applicants and discussions with the designers and DOE regarding their proposed approaches to addressing key issues. Issues included accident selection for small modular reactors. With respect to this issue, the staff noted:

"In the August 2008 NGNP Licensing Strategy, the Commission stated that licensing-basis event categories (i.e., abnormal occurrences, design-basis accidents, and beyond-design basis accidents) would be established based on the expected probability of event occurrence. However, selection of licensing basis events within each category would be performed using deterministic 
engineering judgment complemented by insights from the NGNP PRA. In general, the NRC staff expects to apply this approach to all SMRs.

Although identification of many accident scenarios will likely be straightforward, the application of certain scenarios may require Commission consideration. For example, designers of HTGRs have previously proposed that the failure of the vessel or piping connecting the reactor vessel and steam generator vessel need not be considered as a design basis event. In addition, although the Commission has previously stated that certain events should be addressed for non-LWR designs, subsequent research and evaluations may challenge the need to analyze these low probability events."

The announced NRC staff plans to develop proposed resolutions to the issues in SECY 2010-0034 by continuing to obtain information from DOE, potential design and license applicants, and other sources; identifying and developing proposals for the resolution of policy issues; and where appropriate, preparing papers proposing resolutions of these issues with recommendations for consideration and approval by the Commission. This approach is a reasonable model for non-LWRs generally, whether large or small.

In SECY-16-0012 ${ }^{[20]}$ the NRC staff recommended that design specific DBA mechanistic source terms can be used to address siting and emergency planning requirements for SMRs including those based on nonLWR technology. Importantly this SECY recommends that design-specific DBA source terms used be based on accidents involving a single module:

"The siting dose criteria are expected to be evaluated through DBA dose analyses on a perreactor basis, even for multi-module plants. This is because of the design protection against external events that may affect more than one module concurrently, separation and independence of the modules' systems, structures and components and safety functions, and design against common cause failures among modules, in accordance with GDCs 2, 4, and 5. This means that the siting of a multi-module plant, including the determination of the EAB, LPZ and population center distances, is currently expected to be based upon the evaluation of a single reactor."

This part of the precedent review is helpful in identifying the need for and potential benefits of a riskinformed approach that is capable of identifying an appropriate set of LBEs for advanced non-LWRs employing a modular design approach. Developing the means of evaluating multi-module configurations and other onsite non-reactor risk also provides flexibility to designers regarding how to best configure the plant for economic as well as safety considerations. In addition, it is necessary to incorporate appropriate ground rules for development and review of reactor-specific and scenario specific source terms.

\section{A.1.1.3 NRC GUIDANCE}

\section{A.1.1.3.1 Standard Review Plan (NUREG-0800)}

NUREG-0800, "Standard Review Plan (SRP) Chapter 15.0,"[3] identifies the types of AOOs and DBAs that must be postulated for LWRs. Hence, its review is relevant to developing a process for selecting LBEs.

SRP Chapter 15.0 includes a listing of generic LWR events given as example events and the applicant is directed to propose the design specific AOOs and DBAs along with the design description for the NRC staff to review. There was no guidance or acceptance criteria identified that would ensure that the "limiting" AOOs and DBAs appropriate and applicable to the design are selected for the deterministic safety analysis. A design specific version of the SRP has been developed for the NuScale SMR but the 
corresponding Section 15.0 does not indicate or refer to a method to identify DBAs or AOOs that are specific for that design nor acceptance criteria to ensure that the "limiting" events have been selected. There is no comparable guidance included for non-LWRs.

In Section 19.0 of the SRP ${ }^{[19]}$ includes several sections of regulatory guidance for the technical adequacy of the PRA required to be part of the license application that appear to be relevant to advanced non-LWR designs that employ passive safety features and modular reactor designs. These sections offer limited guidance for the probabilistic inputs to selecting LBEs.

Regarding PRAs performed for passive designs, the following review guidance is provided.

"Design-Specific PRA (Procedures Specific to Passive Designs)

1. The issue of T-H uncertainties in passive plant designs arises from the passive nature of the safety-related systems used for accident mitigation. Passive safety systems rely on natural forces, such as gravity, to perform their safety functions. Such driving forces are small compared to those of pumped systems, and the uncertainty in their values, as predicted by a best-estimate T-H analysis, can be of comparable magnitude to the predicted values themselves. Therefore, some accident sequences with a frequency high enough to impact results, but not predicted to lead to core damage by a best- estimate T-H analysis, may actually lead to core damage when PRA models consider T- H uncertainties. One approach to addressing this issue is to perform sensitivity studies to see the effect of assuming bounding values for T-H parameters on success criteria and performing studies of the sensitivity of changes in success criteria on $C D F$.

A. The reviewer assures that the applicant has (1) identified all key T-H parameters that could affect the reliability of a passive system and introduce uncertainty into the determination of success criteria, and (2) accounted for the uncertainty in the analyses that establish the success criteria.

$B$. The reviewer examines the results of any sensitivity studies performed by the applicant and the choice of T-H accident analysis codes used to perform such studies. Applicants frequently use the Modular Accident Analysis Program (MAAP) code for such studies. The staff is aware of T-H modeling issues with the code that could compromise its ability to confirm the validity of the PRA success criteria involving minimal sets of mitigating equipment. Use of this code is acceptable only if sufficient benchmarking studies have been done which compare MAAP results with those of a T-H code the staff has reviewed and approved and show that MAAP is able to capture the important T-H phenomena and the timing of such phenomena in simulations of accident sequences included in the PRA. If a small set of accident scenarios is used in the studies, the reviewer confirms that the applicant has provided an adequate rationale for its selection of scenarios, including a discussion of the criteria used for selection.

2. For passive plant designs, the staff reviews the applicant's use of the PRA to identify "nonsafety-related," SSCs that require regulatory treatment (i.e., to support the RTNSS program). Specifically this includes the following evaluations performed by the applicant as described in SRP 19.3:

A. Evaluation of the risk significance of nonsafety systems using the Focused PRA

$B$. Evaluation of uncertainties associated with assumptions made in the PRA models of passive systems

C. PRA initiating event frequency evaluation" 
Although this guidance was developed for LWR designs, it provides useful guidance for PRA reviews for advanced non-LWRs that employ passive safety features. Because the LMP approach to selecting LBEs is based in part on information derived from the PRA, consideration of the response of passive safety features to LBEs will be an important element of the LBE selection process for non-LWRs that employ passive safety features.

The SRP for Chapter 19 also includes the following guidance for PRA reviews for modular integral pressurized water reactors that employ modular reactor designs.

"Design-Specific PRA (Procedures Specific to Integral Pressurized Water Reactors)

1. For small, modular integral pressurized water reactor designs, the staff reviews the results and description of the applicant's risk assessment for a single reactor module; and, if the applicant is seeking approval of an application for a plant containing multiple modules, the staff reviews the applicant's assessment of risk from accidents that could affect multiple modules to ensure appropriate treatment of important insights related to multi-module design and operation.

The staff will verify that the applicant has:

- $\quad$ Used a systematic process to identify accident sequences, including significant human errors, that lead to multiple module core damages or large releases and described them in the application

- $\quad$ Selected alternative features, operational strategies, and design options to prevent these sequences from occurring and demonstrated that these accident sequences are not significant contributors to risk. These operational strategies should also provide reasonable assurance that there is sufficient ability to mitigate multiple core damages accidents."

The above guidance is relevant to advanced non-LWR designs that include modular reactors for the PRA inputs to selecting LBEs.

\section{A.1.1.3.2 NUREG-1860 Feasibility Study for a Risk-Informed and Performance-Based Regulatory Structure for Future Plant Licensing ${ }^{[21]}$}

NUREG-1860 is clearly the most relevant document that has been identified in providing a possible methodology for selection of LBEs as well as for formulation of reactor-specific regulatory requirements for advanced non-LWRs. The citations in this report are focused on event sequence identification and derivation of design basis events for specialized evaluation. Other topics relevant to different topics will be included in subsequent LMP reports.

The objectives of the framework include:

"Risk-informed - Ensure that risk information and risk insights are integrated into the decision making process such that there is a blended approach using both probabilistic and deterministic information.

Performance-based - When implemented, the guidance and criteria produce a set of safety requirements that are based on plant performance, and do not use prescriptive means for achieving its goals. 
Defense-in-depth-Defense-in-depth is an integral part of the framework such that uncertainties are accounted for in the requirements for design, construction, and operation.

Flexible-The framework should, allow the licensing process to support reactors of diverse designs and be developed in such a manner that, as new information and knowledge are gained, changes to the regulatory structure can be implemented effectively and efficiently."

The framework was developed from the top down starting with the Atomic Energy Act and includes the following elements:

"Element 1: Goals and Expectations: These start with the Atomic Energy Act principle of providing adequate protection of public health and safety and NRC's expectations for safety security and preparedness. Safety expectations are anchored to the Safety Goal policy and $N R C$ 's expectations that advanced reactors will provide enhanced margins of safety and comply with the Safety Goal policy with the QHOs representing the level of safety intended to achieve. $N R C$ 's security expectations are that advanced reactors will provide enhanced margins of safety and utilize simplified, inherent, passive or other innovative means to accomplish their safety and security functions.

Element 2: Defense-In-Depth: A core principle of the NRC's safety philosophy has always been the principle of defense-in-depth. This principle remains basic to the safety, security, and preparedness expectations in the framework. The ultimate purpose of defense-in-depth is to compensate for uncertainty (e.g., uncertainty due to lack of operational experience with new technologies and new design features, uncertainty in the type and magnitude of challenges to safety). In licensing future reactors, the treatment of uncertainties will play a key role in ensuring that safety limits are met and that the design is robust for unanticipated factors.

Element 3: Safety Fundamentals: This element provides the path, or process, from the high level goals and expectations to actually establishing specific requirements.... The process chosen to initially identify and define the requirements and regulations needs to implement the safety, security, and preparedness expectations and ensure protection of the public health and safety. Safety fundamentals have been defined, using a defense-in-depth approach, in the form of protective strategies that, if met, will ensure the protection of the public health and safety with a high degree of confidence.... A top-down analysis of each protective strategy leads directly to a categorization of the kinds of requirements that can ensure that the protective strategies are met.

Element 4: Licensing Basis: A major goal is that the regulatory licensing basis be riskinformed.... The current regulatory structure is deterministic and is being modified in places to incorporate risk insights. A risk-informed regulatory structure should integrate risk from conception...In the framework, probabilistic criteria integrated with deterministic criteria based on plant specific considerations are used to establish potential new requirements....In using a probabilistic process, confidence in the technical acceptability becomes a key factor. Therefore, the technical acceptability of the PRA is part of this element.

The licensing basis criteria are parallel and complementary with the Protective Strategies, in support of the NRC's defense-in-depth expectations, as shown in Figure 2-5. The probabilistic criteria include compliance with the quantitative health objectives (QHOs) of the NRC's safety goals. 
The framework establishes probabilistic criteria to ensure that:

- The integrated plant risk is acceptable in terms of the QHOs of the NRC's safety goal policy statement,

- A frequency consequence $(F-C)$ curve is developed ... that together with the plant PRA is used to select licensing basis events.

- The selection of those events that are used to establish the licensing basis of the design (licensing basis events or LBEs) is carried out in a risk-informed manner,

- The LBEs meet the F-C curve with margin, and

- The safety classification of systems, structures, and components (SSCs) reflects their importance in reducing plant risk.

In selecting both the LBEs and the safety significant SSCs, defense-in-depth measures are incorporated, but, in addition, the risk information from the PRA is used to focus attention on the risk-significant aspects of the design.

LBEs derived from the PRA need to meet stringent probabilistic acceptance criteria and, depending on their frequency, need to meet additional deterministic (defense-in-depth) criteria. In this manner, the LBEs provide additional assurance that the design has adequate defense-indepth in the form of sufficient margin to account for uncertainties. The LBEs also include a deterministically selected event, used in assessing site suitability.

Element 5: Integrated Process: The process for identifying the requirements begins with the protective strategies. Each one is examined with respect to what are the various threats or challenges that could cause the strategy to fail. These challenges and threats are identified using a logic tree to perform a "systems analysis" of the strategy to identify potential failures. The defense-in-depth principles are then applied to each protective strategy. Defense-in-depth measures are identified which should be incorporated into the requirements to help prevent protective strategy failure. This approach forms the process for the selection of "topics." Requirements are then identified for each topic.

Part of the process involves development of guidance to be used for actually writing the requirements. This guidance addresses writing the requirements in a performance-based fashion, incorporating lessons learned from past experience, and utilizing existing requirements and guidance, where practical. The guidance also ensures that the probabilistic process for establishing the licensing basis are incorporated. All of the above are integrated and results in a set of potential requirements which serve to illustrate and establish the feasibility of developing a risk-informed and performance-based licensing approach."

The framework set forth in NUREG-1860 has influenced the LMP selection approach for selecting LBEs for advanced non-LWRs. This LBE approach differs from that of NUREG-1860 in some important instances as well. This is discussed more fully in Section 4 of this report.

NUREG-1860 includes guidance for performing PRAs on advanced non-LWRs and provides recommendations for PRA technical adequacy requirements for PRAs used to support the licensing bases. This aspect of the document is reviewed in the companion LMP report on PRA. 


\section{A.1.1.3.3 NUREG-2150 A Proposed Risk Management Regulatory Framework ${ }^{[22]}$}

In early 2011, an NRC Commissioner led a Risk Management Task Force (RMTF) to evaluate how the agency should be regulating 10 to 15 years in the future. The RMTF was chartered:

"to develop a strategic vision and options for adopting a more comprehensive and holistic riskinformed, performance-based regulatory approach for reactors, materials, waste, fuel cycle, and transportation that would continue to ensure the safe and secure use of nuclear material."

The RMTF report developed a series of findings and recommendations covering the full scope of nuclear facilities including existing reactors and fuel cycle facilities and advanced non-LWRs to be licensed in the future. One of the key findings was that risk management should be stated as the NRC's objective according to a proposed framework shown in Figure A-1 (reproduced from Figure ES-1 in NUREG2150). The first recommendation was that:

"The NRC should formally adopt the proposed Risk Management Regulatory Framework through a Commission Policy Statement."

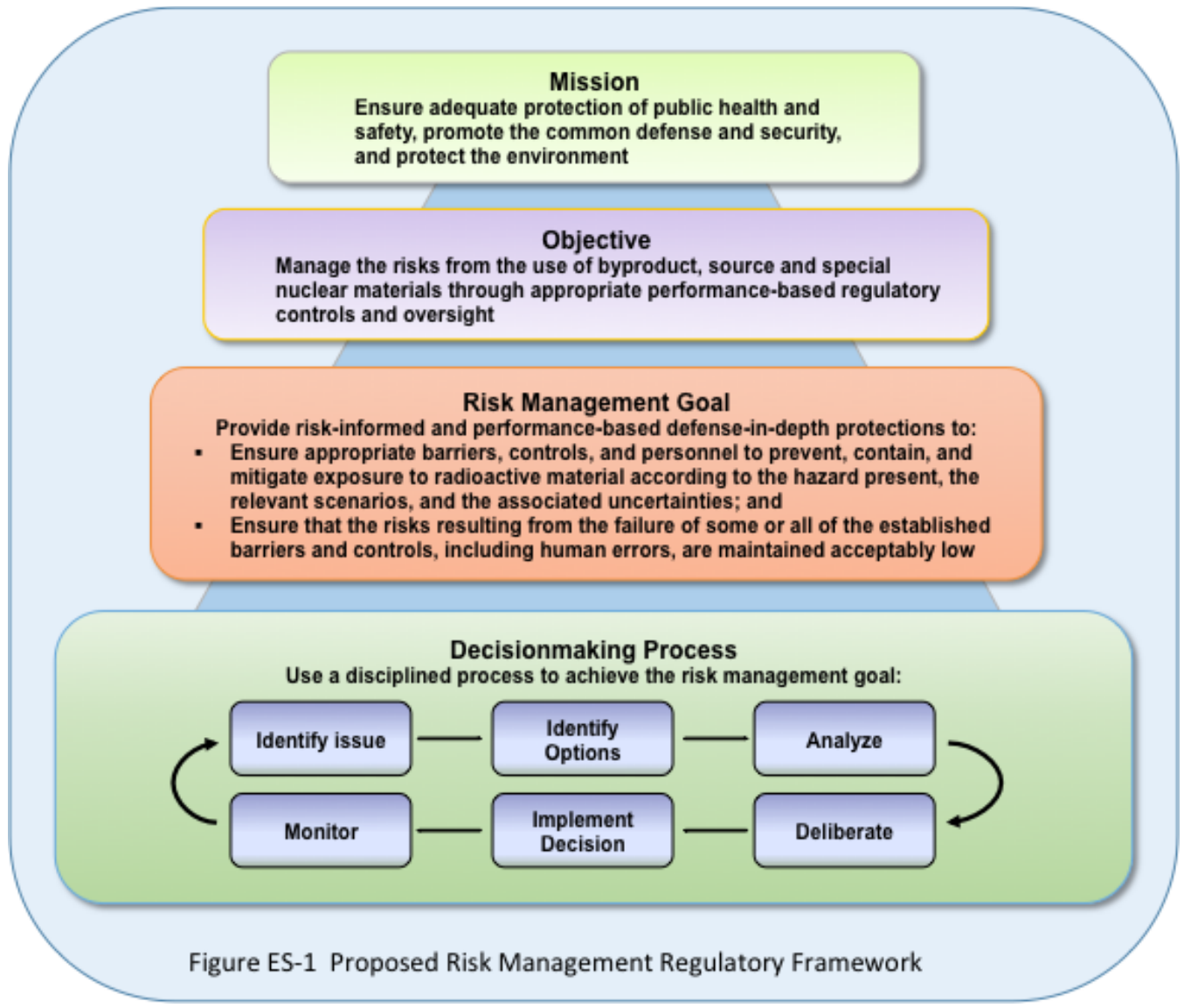

Figure A-1. Regulatory Framework Proposed in NUREG-2150

Specific recommendations were made for implementing the framework in Figure A-1 for Generation IV reactors which encompass the advanced non-LWR designs intended for the LBE selection process outlined in this report. Those recommendations, which are listed in Table A-2, were considered in the development of the LBE selection process proposed in Section 4 of this report. 
Table A-2. NRC Risk Management Task Force Recommendations for Generation IV Reactors

\begin{tabular}{|c|c|}
\hline $\begin{array}{l}\text { RMTF Recommendation } \\
\text { for Generation IV Reactors }\end{array}$ & $\begin{array}{c}\text { Referenced RMTF Recommendation } \\
\text { for Power Reactors }\end{array}$ \\
\hline $\begin{array}{l}\text { GIV-R-1: For Generation IV reactors, the RMTF } \\
\text { recommends that the concept of design-basis } \\
\text { accidents be maintained, but the NRC should be } \\
\text { amenable to and promote, where practical, the } \\
\text { adoption of more risk-informed approaches for the } \\
\text { selection of relevant scenarios (e.g., alternatives to } \\
\text { the single failure criterion) for design-basis } \\
\text { accidents. }\end{array}$ & N/A \\
\hline $\begin{array}{l}\text { GIV-R-2: Apply Recommendation PR-R-2 } \\
\text { (design-enhancement category) to Generation IV } \\
\text { reactors. }\end{array}$ & $\begin{array}{l}\text { PR-R-2: The NRC should establish through rulemaking } \\
\text { a design-enhancement category of regulatory treatment } \\
\text { for beyond-design-basis accidents. This category should } \\
\text { use risk as a safety measure, be performance-based } \\
\text { (including the provision for periodic updates), include } \\
\text { consideration of costs, and be implemented on a site- } \\
\text { specific basis. }\end{array}$ \\
\hline $\begin{array}{l}\text { GIV-R-3: Apply Recommendation PR-R-3 } \\
\text { (include external events in design-enhancement } \\
\text { category) to Generation IV reactors. }\end{array}$ & $\begin{array}{l}\text { PR-R-3: The NRC should reassess methods used to } \\
\text { estimate the frequency and magnitude of external } \\
\text { hazards and implement a consistent process that includes } \\
\text { both deterministic and PRA methods. Consideration of } \\
\text { the risks from beyond-design-basis external hazards } \\
\text { should be included in the design-enhancement category } \\
\text { described in Recommendation PR-R-2. }\end{array}$ \\
\hline $\begin{array}{l}\text { GIV-R-4: Apply Recommendation PR-R-4 } \\
\text { (periodically evaluate new information regarding } \\
\text { external hazards) to Generation IV reactors. }\end{array}$ & $\begin{array}{l}\text { PR-R-4: The NRC should establish a program to } \\
\text { systematically collect, evaluate, and communicate } \\
\text { external hazard information. }\end{array}$ \\
\hline $\begin{array}{l}\text { GIV-R-5: Apply Recommendation PR-R-5 (issue } \\
\text { guidance to adopt risk-informed and performance- } \\
\text { based defense-in-depth) to Generation IV reactors. }\end{array}$ & $\begin{array}{l}\text { PR-R-5: The NRC should apply the risk-informed and } \\
\text { performance-based defense-in-depth concept to power } \\
\text { reactors in a more quantitative manner. }\end{array}$ \\
\hline $\begin{array}{l}\text { GIV-R-6: Apply Recommendation PR-R-6 } \\
\text { (develop guidance and consistent approach } \\
\text { between safety and security) to Generation IV } \\
\text { reactors. }\end{array}$ & $\begin{array}{l}\text { PR-R-6: The NRC should develop and implement } \\
\text { guidance for use in its security regulatory activities that } \\
\text { uses a common language with safety activities and } \\
\text { harmonizes methods with risk assessment and the } \\
\text { proposed risk-informed and performance-based defense- } \\
\text { in-depth framework. }\end{array}$ \\
\hline
\end{tabular}

These recommendations are consistent with the LMP goal of using a TI-RIPB approach to make licensing decisions for advanced non-LWRs, including the selection of LBEs and application of defense-in-depth principles that span the full spectrum hazards, frequencies, and consequences.

\section{A.1.1.3.4 Fukushima Accident and NRC Near-Term Task Force (NTTF) Report ${ }^{[23]}$}

On March 11, 2011, the Great East Japan Earthquake occurred 231 miles northeast of Tokyo off the coast of Honshu Island. This earthquake resulted in the automatic shutdown of 11 nuclear power plants at four sites along the northeast coast of Japan (Onagawa 1, 2, and 3; Fukushima Daiichi 1, 2, and 3; Fukushima Dai-ni 1, 2, 3, and 4; and Tokai 2). The earthquake precipitated a large tsunami that is estimated to have exceeded 14 meters (45 feet) in height at the Fukushima Daiichi Nuclear Power Plant site. The earthquake and tsunami produced widespread devastation across northeastern Japan, resulting in approximately 25,000 people dead or missing, displacing many tens of thousands of people, and 
significantly impacting the infrastructure and industry in the northeastern coastal areas of Japan. The site inundation at Fukushima Daiichi led to a severe core damage accident on three of the six reactor units, and containment breach on at least one unit, due to a prolonged loss of $\mathrm{AC}$ and $\mathrm{DC}$ power due to flood damage to onsite emergency diesel-generators and electrical switchgear. The management of the accident, which was successful in preventing significant off-site radiological exposures to the public, was complicated by the concurrent needs to manage the accident and to protect the remaining units and associated spent fuel storage facilities.

In July 2011, the NRC issued its Near Term Task Force (NTTF) Report with recommendations for enhancing reactor safety. It included the greater use of risk considerations and defense-in-depth in its final report. There are 12 specific recommendations are organized into the following topics.

\section{"Clarifying the Regulatory Framework}

1. The Task Force recommends establishing a logical, systematic, and coherent regulatory framework for adequate protection that appropriately balances defense-in-depth and risk considerations. (Section 3)

\section{Ensuring Protection}

2. The Task Force recommends that the NRC require licensees to reevaluate and upgrade as necessary the design-basis seismic and flooding protection of structures, systems, and components for each operating reactor. (Section 4.1.1)

3. The Task Force recommends, as part of the longer term review, that the NRC evaluate potential enhancements to the capability to prevent or mitigate seismically induced fires and floods. (Section 4.1.2)

\section{Enhancing Mitigation}

4. The Task Force recommends that the NRC strengthen station blackout mitigation capability at all operating and new reactors for design-basis and beyond-design-basis external events. (Section 4.2.1)

5. The Task Force recommends requiring reliable hardened vent designs in boiling water reactor facilities with Mark I and Mark II containments. (Section 4.2.2)

6. The Task Force recommends, as part of the longer term review, that the NRC identify insights about hydrogen control and mitigation inside containment or in other buildings as additional information is revealed through further study of the Fukushima Dai-ichi accident. (Section 4.2.3)

7. The Task Force recommends enhancing spent fuel pool makeup capability and instrumentation for the spent fuel pool. (Section 4.2.4)

8. The Task Force recommends strengthening and integrating onsite emergency response capabilities such as emergency operating procedures, severe accident management guidelines, and extensive damage mitigation guidelines. (Section 4.2.5)

\section{Strengthening Emergency Preparedness}

9. The Task Force recommends that the NRC require that facility emergency plans address prolonged station blackout and multiunit events. (Section 4.3.1)

10. The Task Force recommends, as part of the longer term review, that the NRC pursue additional emergency preparedness topics related to multiunit events and prolonged station blackout. (Section 4.3.1)

11. The Task Force recommends, as part of the longer term review, that the NRC should pursue emergency preparedness topics related to decision making, radiation monitoring, and public education. (Section 4.3.2)

Improving the Efficiency of NRC Programs 
12. The Task Force recommends that the NRC strengthen regulatory oversight of licensee safety performance (i.e., the Reactor Oversight Process) by focusing more attention on defense-indepth requirements consistent with the recommended defense-in-depth framework. (Section 5.1)”

These recommendations identify the need to address the risk of multiunit accidents via enhancements to emergency planning, ensure adequate protection for seismic events and external flooding, and to provide the capabilities for coping with an extended loss of AC power. These recommendations are supported by the NTTF finding recognizing the complementary roles of defense-in-depth and PRA.

"The Task Force finds that the Commission's longstanding defense-in-depth philosophy, supported and modified as necessary by state-of-the-art probabilistic risk assessment techniques, should continue to serve as the primary organizing principle of its regulatory framework. The Task Force concludes that the application of the defense-in-depth philosophy can be strengthened by including explicit requirements for beyond-design-basis events."

This document includes general guidance on the importance of a modern RIPB framework and the importance of determining a well-structured and set of LBEs to base design conditions for SSCs.

\section{A.1.1.3.5 NRC Guidance for Performance-Based Regulation ${ }^{[24]}$}

An objective for the LMP is to define an approach to selecting LBEs that is both risk-informed and performance-based (RIPB). Regulatory documents reviewed in previous sections contain useful guidance for how LBEs may be risk-informed but do not explicitly define what additional characteristics may be necessary to classify the approach as performance-based. Guidance on criteria for defining performancebased alternatives in regulatory decision making is found in NUREG/BR-0303. ${ }^{[24]}$

That document provides guidance on a process for developing performance-based alternatives to more prescriptive approaches for regulatory decision making. The U.S. Nuclear Regulatory Commission (NRC) Management Directive 6.3, "Rulemaking," calls for the consideration of a performance-based alternatives. Such alternatives differ significantly from a prescriptive approach by providing a focus on measurable performance attributes and outcomes. Such alternatives have the potential to improve the objectivity and transparency of NRC decision making and provide greater flexibility to designers and licensees, reduce licensee burden, and promote safety by focusing on safety-successful outcomes.

The process in Management Directive 6.3 is set up in terms of five steps which are intended to provide the information to formulate a performance-based alternative to a prescriptive requirement. The five steps in the process are:

1. Defining the regulatory issue and its context

2. Identifying the safety functions

3. Identifying safety margins

4. Selecting performance parameters and criteria

5. Formulating a performance-based alternative

This document provides useful guidance in developing the risk-informed and performance-based process for selecting LBEs that is described in Section 4 of this report. The use of F-C Targets in this report is an example of PB practice at the plant level. Additional consideration of PB practices will also be included in companion LMP reports on RIPB SSC classification and risk-informed applications of PRA beyond LBE selection. 


\section{A.1.2 INTERNATIONAL GUIDANCE FOR LICENSING BASIS EVENT SELECTION}

\section{A.1.2.1 IAEA SAFETY OF NUCLEAR POWER PLANTS: DESIGN NSR-1 ${ }^{[38]}$}

The International Atomic Energy has published a number of reports on the safety of nuclear power plants including NSR-1 on the design aspects of nuclear safety. This report sets forth basic safety principles to be considered in design to ensure defense-in-depth and high-level objectives of the deterministic and probabilistic safety analyses that should be performed to demonstrate adequate safety. The deterministic safety analysis starts with the identification of "Postulated Initiating Events (PIEs)" defined as an event identified during design as capable of leading to anticipated operational occurrences or accident conditions. The following excerpt shows the high level and qualitative character of the guidance for identifying PIEs:

"A full range of events needs to be postulated in order to ensure that all credible events with potential for serious consequences and significant probability have been anticipated and can be withstood by the design of the plant. There are no firm criteria to govern the selection of PIEs; rather the process is a combination of iteration between the design and analysis, engineering judgment and experience from previous plant design and operation. Exclusion of a specific event sequence needs to be justified."

Although this document provides useful insights for the design of a nuclear power plant, it was specifically developed for currently operating LWRs and does not appear to provide additional guidance for non-LWRs beyond that which can be similarly gleaned from the NRC SRP.

\section{A.1.2.2 UNITED KINGDOM SAFETY ASSESSMENT PRINCIPLES FOR NUCLEAR FACILITIES ${ }^{[39]}$}

The Office for Nuclear Regulation (ONR) is the independent regulator of nuclear safety and security across the United Kingdom. ONR's inspectors use the Safety Assessment Principles (SAPs) described in that document, together with supporting Technical Assessment Guides (TAGs), to guide their regulatory judgments and recommendations when undertaking technical assessments of nuclear site licensees' safety submissions. Supporting these is the legal duty on licensees to reduce risks so far as is reasonably practicable (ALARP) principle informs the use of these SAPs. In addition, the SAPs are used to guide our assessments of proposed new nuclear facilities designs that may come forward for eventual construction at sites in the U.K.

What is noteworthy in this document is that the SAPs have already been demonstrated to be technologyinclusive and more performance-based that NRC's framework. Earlier versions of these SAPs were used to license two different types of graphite moderated gas-cooled reactors (MAGNOX, and AGR) and a current generation Westinghouse PWR at Sizewell. The 2014 version of the SAPs reflects revisions to account for lessons from the Fukushima Daiichi accident. There are currently SAP-based licensing reviews underway at various stages for new, diverse reactor facilities that may be added to existing sites covering the following designs: a two-unit GE-Hitachi ABWR plant, a two-unit AREVA EPR plant, a three-unit Westinghouse AP1000 plant, and a two-unit CGN HPR-1000 plant. In addition, there are prelicensing generic discussions underway for an Integral Pressurized Water Reactor (iPWR)-based SMR designs and a PRISM liquid metal fast reactor.

The SAPs provide useful guidance for the selection of LBEs in this project because they contain the following elements: 
- Inclusion of a risk-informed blend of deterministic safety analysis, probabilistic safety analysis, and severe accident analysis

- Numerical criteria for selection of DBAs based on frequency of occurrence and resulting from a fault analysis that includes probabilistic and deterministic inputs. Internally initiated accidents less than $10^{-5}$ per year and accidents initiated by external hazards conservatively estimated to be less than $10^{-4}$ per year may be excluded from DBAs subject to assurance that risk targets (See BSO and BSL below) and certain deterministic requirements are met.

- Requirements for using a PSA* to balance the risk contributions across the design so as to ensure there are no "weak links" in the design that dominate the risks.

- Numerical frequency-consequence criteria for evaluating the acceptability of risks. These criteria are framed in terms of Basic Safety Objectives (BSOs) and Basic Safety Limits (BSLs). Risks below the BSOs are regarded as "broadly acceptable," those between the BSOs and BSLs "tolerable," and those exceeding the BSLs "unacceptable." There are BSOs and BSLs for normal operation, design basis accidents, and beyond design basis accidents. Depending on the specific target the consequence limits are applied to workers on-site as well as off-site public exposures.

- In addition to the BSOs and BSLs the SAPs include a requirement to maintain risk levels to as low as reasonably practicable (ALARP) which means that if there are cost effective ways to reduce risk, those means should be implemented regardless of the risk levels relative to BSOs and BSLs.

- When the SAPs are applied to a Generic Design Assessment, which roughly corresponds to a U.S. Design Certification in scope, the frequency basis of the BSO and BSL numerical targets are applied on a reactor-year basis for a generic site. Then when the facility is added to a site, there are separate frequency-consequence criteria applied on an integrated site basis. In this part of the site the equivalent of an integrated site wide assessment of risk is required.

The UK SAPs were found to be very useful in defining the approach to selecting LBEs described in Section 4 including the use of frequency and dose criteria to evaluate the risk of LBEs. This work was also found to be useful in develop the PRA approach that is discussed more fully in a companion report. Section A.1.4 provides more information on the numerical risk targets employed within the SAPs. The SAPs are also informative with respect to performance-based criteria development generally. A RIPB SSC classification LMP report will discuss this further.

\section{A.1.3 HISTORICAL PRECEDENTS FOR ADVANCED NON-LWRS}

\section{A.1.3.1 MODULAR HIGH-TEMPERATURE GAS-COOLED REACTOR PRE-APPLICATION REVIEW}

In the late 1980s and early 1990s, the NRC conducted a pre-application review of the modular hightemperature gas-cooled reactor (MHTGR) at the request of the DOE. The MHTGR is a graphite moderated, prismatic fueled, helium cooled, high temperature reactor with passive heat removal and reactivity control features.

DOE proposed a systematic, structured method for selecting LBEs that used a top-down approach based on top-level regulatory criteria and PRA. This approach was subsequently built upon in the Exelon, PBMR and DOE NGNP programs, but the MHTGR represents the fullest demonstration of the approach with an actual design, a design specific PRA, and an extensive NRC pre-application to the point of a draft SER, discussed further below.

\footnotetext{
* IAEA and SAP documents use the term Probabilistic Safety Analysis (PSA) to mean the same thing as Probabilistic Risk Analysis (PRA) according to the IAEA safety term glossary in Reference [38].
} 
The scope of the NRC and NRC contractor review for the MHTGR included:

- A Preliminary Safety Information Document (PSID) ${ }^{[40]}$ that included a design description that roughly corresponds to the Safety Analysis Report format in RG 1.70.

- An MHTGR design specific PRA ${ }^{[41]}$ that included:

$\circ$ MHTGR-specific initiating events, event sequences, and end states

- Fault tree models and data to estimate event sequence frequencies

- Plant transient response analysis for each event sequence

O Offsite dose consequences for each MHTGR-specific release category.

- A Risk-informed licensing approach based on:

- Then current LWR requirements and the NRC safety goals

- Top Level Regulatory Criteria derived from NRC regulations interpreted in the form of a frequency-consequence curve for evaluating the risk significance of LBEs

- A set of MHTGR design specific LBEs* derived from the MHTGR PRA based on probabilistic and deterministic criteria, including AOOs, DBEs, BDBEs, and DBAs.

- A method for selecting safety-related SSCs based on probabilistic and deterministic criteria and application of the method to the MHTGR

- Regulatory design criteria for safety-related SSCs during MHTGR-specific LBEs in the performance of MHTGR-specific safety functions.

- Probabilistic and Deterministic Safety analyses for all AOOs, DBEs, selected BDBEs, and DBAs.

The NRC published preliminary results of its MHTGR review in NUREG-1338, "Draft Pre-application Safety Evaluation Report for the Modular High-Temperature Gas-Cooled Reactor." ${ }^{[42]}$ In Sections 3.1.2 of NUREG-1338, the NRC stated that:

"The staff concludes that the DOE's approach is a systematic and useful approach for design of a nuclear plant. However, it is not an adequate replacement for the application of NRC's regulatory approach to the safety and licensing review. Specifically, the staff found, as a result of review of the MHTGR, that many regulatory criteria (10 CFR) and much Standard Review Plan (NRC report NUREG-0800) guidance are applicable to the MHTGR, and the application of these criteria is necessary to ensure that the MHTGR achieves at least an equivalent level of safety as that of current-generation LWRs."

In the course of this review, the NRC proposed some additional deterministically selected LBEs be added to the safety evaluation. In response to this, these events were added to the evaluation with an estimate of the frequency of occurrence and an evaluation that no cliff edge effects were identified that would exceed the F-C Targets. No risk significant sequences were identified from these added events. In addition, the NRC review commented that a small number of SSCs that were classified as non-safety related should be added to the safety related list. However, the vast majority of the SSC safety classifications were accepted.

\section{A.1.3.2 EXELON PBMR PRE-APPLICATION REVIEW}

In 2001 to 2002, the NRC staff conducted a pre-application review of the PBMR design at the request of Exelon. As part of the pre-application engagement with NRC, Exelon proposed a RIPB process similar to the MHTGR process. MHTGR examples were used as well, similar to the LMP examples. In a project

\footnotetext{
* In the MHTGR submittals, BDBEs were referred to as "Emergency Planning Basis Events (EPBEs), and Design Basis Accidents were referred to a "Safety Related Design Conditions" (SRDCs).
} 
closeout letter* to Exelon dated March 26, 2002, the NRC staff provided its initial assessment, amongst other things, of the licensing approach proposed by Exelon, including the use of Top Level Regulatory Criteria (TLRC $\left.{ }^{\dagger}\right)^{[48]}$ With respect to selection of TLRC, the NRC staff stated:

"The staff notes that plotting of TLRC is useful to illustrate bounding criteria and safety margins. However, the licensing basis is the set of requirements that are applied to the safety-related equipment to meet the LBEs (or other special regulatory objectives such as anticipated transients without scram (ATWS) or station black-out (SBO)); simply falling within the plot of the TLRC does not in itself constitute a complete licensing basis. Moreover, while the PRA confirms risk insights for a design, and can be used for other purposes as noted above, licensing activities will be a mix of "deterministic" analysis supplemented with risk insights. The lack of operational data for some of the unique PBMR SSCs makes complete reliance on PRA difficult"

With respect to selection of LBEs, the NRC staff stated in its letter:

"In the SRM for SECY-93-092, "Issues Pertaining to the Advanced Reactor (PRISM, MHTGR, and PIUS) and CANDU 3 Designs and their Relationship to Current Regulatory Requirements, " issued April 8, 1993, regarding accident selection and evaluation, the Commission approved the staff recommendation that events and sequences be selected deterministically and use conservative assumptions, and be supplemented with insights from the PRA for the specific design. In Exelon's August 31, 2001, document containing its proposed licensing approach, Exelon appears to be using probabilistic criteria to select AOOs, DBEs, and EPBEs. However, from verbal interactions with Exelon, the staff believes that the candidate LBEs which will be considered for application within the framework of the TLRC will first be established deterministically, and will then be assessed and compared to the TLRC using risk insights. To the extent Exelon adheres to such an approach, the staff believes it would be consistent with previous Commission guidance."

Following the Exelon review, the NRC staff provided the Commission a status report on the policy implications from licensing non-LWR designs and the staff's plans for seeking Commission guidance on resolving the issues. Three overarching policy issues and four policy issues of a more specific nature were discussed in SECY-02-0139. Of the seven issues, Issue 4, "'To what extent should a probabilistic approach be used to establish the plant licensing basis?") specifically relates to LBE selection. The Commission approved the staff"s recommendation in the "Staff Requirements Memorandum on SECY-03-0047" to allow the use of a probabilistic approach in the identification of events (See Section A.1.2.1 of this report for additional details).

The NRC findings in these reviews for the DOE MHTGR and the Exelon PBMR licensing approaches have been considered in the approach that is described in Section 4. For the LMP, as was the case with the MHTGR, there is no intent to limit the licensing basis to just meeting the frequency-dose criteria derived from the TLST. As with currently licensed LWRs, the RIPB approach includes deterministic design criteria for barriers and SSCs that are necessary and sufficient to justify the assumptions made in

\footnotetext{
${ }^{*}$ Exelon terminated the PBMR pre-application program as part of a corporate restructuring effort to refocus on core businesses. As a part equity owner in PBMR Pty LTD, this was one of many non-core businesses sold or stopped.

$\dagger$ The LMP equivalent to the TLRC are referred to as "Top Level Safety Targets" (TLST) to emphasize that the frequency-dose criteria are design targets and not regulatory limits.
} 
the PRA on the capabilities and reliabilities of SSCs in the prevention and mitigation of accidents. The collection of report to be presented on the TI-RIPB approach, including this report and others on SSC safety classification and defense-in-depth will clarify this key point.

Following the closure of the Exelon PBMR pre-application program, PBMR Pty LTD (PBMR) initiated a pre-application program in early 2004 in its own name. Four RIPB white papers were submitted: PRA; LBE Selection; SSC Classification; and, Defense-In-Depth. This report on LBE selection also draws from the review of those white papers. In its letter of September 24, 2007, the NRC sent Requests for Additional Information (RAIs) on white papers that had been submitted by PBMR to the NRC for review, including the LBE Selection white paper. ${ }^{[45]}$ Responses to these RAIs were provided by PBMR on March 21, 2008. ${ }^{[46]}$ Subsequent to the provision of responses to the RAIs, the PBMR licensing project activities were discontinued in May 2010, and the RAI responses were not reviewed by the NRC. These papers were the forerunners to more recent NGNP papers submitted to NRC as part of the NGNP Licensing Strategy.

\section{A.1.3.3 NEXT GENERATION NUCLEAR PLANT (NGNP) LICENSING APPROACH REVIEW}

In 2005, the U.S. Department of Energy (DOE) established the Next Generation Nuclear Plant (NGNP) Project at the Idaho National Laboratory (INL) following authorization in the EPACT of 2005. This action supported commercial deployment of a high temperature, gas-cooled reactor (HTGR) technology demonstration plant.

The NGNP project included development of a regulatory framework supportive of commercial HTGR deployment. Framework activities were closely coordinated with the NRC staff and focused on adapting existing nuclear power plant regulatory requirements to the needs of NGNP licensing. The approach for this licensing structure was jointly formulated by DOE and NRC and communicated to Congress in 2008.

NGNP examined HTGR licensing precedents and NRC regulations as they relate to the NGNP safety case and associated plant design goals. The scope and results of this examination were coordinated with and reviewed by NRC staff. In 2009, NGNP used this information to develop a strategic implementation plan for establishing the regulatory basis necessary to complete and submit a HTGR license application to NRC. The plan included:

- "Developing the basis for establishing a mechanistic radiological source term (based primarily on particle fuel design and available qualification testing results)

- Preventing/mitigating the release of the radiological source terms to the environment, including methods for the structured and comprehensive identification of licensing basis event sequences, along with establishing multiple radionuclide release barriers

- Developing an updated emergency planning structure that considers collocated industry energy end-users to assure protection of public health and safety in the unlikely event of a radiological release."

A key NGNP methodology in addressing this strategy was to document proposed approaches in a series of complementary pre-licensing white papers. Each white paper included a specific set of outcome objectives that support NGNP licensing and was developed with inputs from DOE and the NGNP 
Licensing Working Group. One of these white papers addressed an approach to selecting LBEs ${ }^{[1]}$ and a related paper on an approach to performing a PRA that provided input to the LBE approach. ${ }^{[62]^{*}}$

In early 2012, NGNP's DOE/INL team and NRC staff jointly identified and agreed to focus on four key licensing framework topics covering sources of significant regulatory uncertainty for the entire HTGR industry. ${ }^{[49]}$ These topics included:

- HTGR containment functional performance

- Licensing basis event selection

- Mechanistic source terms

- Emergency planning

Ensuing interactions resulted in NRC staff drafting initial regulatory positions on the four framework topics and submitted them to the NRC's Advisory Committee on Reactor Safeguards (ACRS) for review in early 2013. ${ }^{[50]}$ Staff findings were then updated and again released in July 2014.

Major items addressed in that NRC staff position report included the following statements relevant to the proposed approach for a risk-informed selection of LBEs:

'The licensing basis event identification and categorization process proposed by NGNP included a frequency versus consequence approach for evaluating postulated event sequences against top level regulatory criteria (primarily offsite dose). Initially, based on public meeting discussions and a draft feedback summary written by NRC staff, this approach appeared to be generally reasonable. However, some members of the staff believed that a supplement was probably necessary to DOE/INL's proposed set of design basis accidents (DBAs). This supplement entailed additional deterministically postulated accidents. NGNP personnel felt that adding events from outside the proposed event selection process created significant uncertainty for the industry. The concept of a supplement was also subject to challenge by ACRS recommendations. This issue (and other related topics) was not addressed in the July 2014 NRC staff position report. The omission on this topic, as well as the overall licensing basis event identification and categorization process in general, was attributed to staff concerns that issuing feedback on the topic now might be inconsistent with ongoing NRC efforts related to post-Fukushima Near Term Task Force (NTTF) Recommendation 1 and subsequent development of a risk management regulatory framework. The proposed mechanistic methodology for defining and evaluating source terms was reasonable to NRC staff.

The staff was receptive to future emergency planning proposals for a probabilistic risk assessment (PRA) informed approach in sizing the emergency planning zone. Proposals might include use of accident dose assessments when determining an appropriate emergency planning zone size (see NRC's SECY 11-0152 [51], which contains a partial response to NGNP white paper proposals). However, clarification beyond SECY 11-0152 was not provided due to the need for Commission action on related policy issues. Further staff evaluation of the NGNP emergency planning approach was curtailed pending availability of more site and plant design information."

\footnotetext{
* As part of the NGNP Licensing Plan, a longer list of white papers, technical and topical reports were intended to be developed on a timeline consistent with a more complete design and licensing application development program. That program did not materialize and the NGNP - NRC refocused their efforts on a select subset of papers to be submitted to NRC.
} 
The ACRS met May 9-10, 2013, to review the NRC staff's assessment of the NGNP key licensing issues identified in the above paragraphs. The ACRS Subcommittee on Future Plant Designs reviewed INL key licensing issue white papers on January 17, 2013, and staff assessments of the INL white papers on April 9, 2013. The following conclusions and recommendations were reached by the ACRS. ${ }^{[50]}$

1. "The staff assessment of the NGNP white papers on key technical issues is appropriate, given the unavailability of many plant-specific design details, such as the selected fuel form (pebble or prismatic) and a complete plant design. The final assessments should be published after the issues raised in Recommendations 2, 3, and 4 are addressed.

2. The assessment documents should be revised to provide clear links to the numerous requests for additional information (RAIs) and responses that were developed during their assessment because the white papers have not been revised to incorporate those agreements.

3. The licensing basis event selection assessment should point out the need to clarify the definition of event sequences and event sequence families to ensure consistency in developing licensing basis events and design basis accidents (DBAs). Incoherent logic in the event trees should be addressed.

4. The staff's suggestion that the final selection of DBAs include postulated deterministic event sequences is inconsistent with a risk-informed framework proposed by the NGNP project and with other on-going NRC activities encouraged by the Commission. Although engineering judgment may be invoked to include postulated deterministic event sequences in the final selection of DBAs, if such sequences are not in the probabilistic risk assessment (PRA), the PRA is incomplete and should be revised to include them. They then can be fully evaluated and considered for inclusion as DBAs."

The approach to selection of LBEs described in Section 4 of this report benefitted from the guidance offered in the NRC and ACRS reviews of the NGNP white papers summarized in the previous paragraphs.

\section{A.1.3.4 PRISM PRE-APPLICATION REVIEW}

Power Reactor Innovative Small Module (PRISM) Liquid-Metal Reactor is part of DOE's advanced liquid-metal reactor program. PRISM is a small, modular, pool-type, liquid-metal (sodium)-cooled reactor. Multiple modules are expected to constitute a power block and multiple power blocks may be combined to constitute a power station of more than $1000 \mathrm{MWe}$. Each reactor module would be a standard design that would be built in a factory and shipped by rail to a site. PRISM uses an advanced metal fuel (plutonium-uranium-zirconium) alloy inside cladding of ferritic steel alloy (HT9) within a fuel rod assembly arrangement. The core structure material is also HT9. Six control rods provide the necessary operational reactivity control. The standard plant design for the PRISM consists of three identical power blocks with a total electrical output rating of 1395 MWe. Each power block comprises three reactor modules, each with an individual thermal rating of $471 \mathrm{MWt}$. Each reactor module is located in its own below-grade silo and is connected to its own intermediate heat transport system and steam generator system. The design includes passive reactor shutdown and passive decay heat removal features.

PRA is employed as a design tool for PRISM. A preliminary PRA was used in the conceptual design phase to define a set of accident sequences from initiating event to radiological release into the environment. PRA is treated as an essential part of the design process providing essential safety inputs to the design. 
The PRISM PRA was used to help ensure completeness in the identification of accident sequences and to rank the sequences in order of their importance based on their expected occurrence frequency and offsite consequences. Licensing basis events were categorized as either Design Basis Accidents or Beyond Design Basis Accidents using the event sequences. All event sequences were considered as candidate LBEs. Specific guiding criteria include:

1. "Event sequences with frequency greater than $10^{-6}$ per reactor-year are within the design basis event envelope. There are four categories of DBEs including "normal operation" including events with frequencies greater than $10^{-1}$ per reactor year, "anticipated events" with frequencies between $10^{-1}$ and $10^{-2}$ per reactor-year, "unlikely events" with frequencies between $10^{-2}$ and $10^{-4}$ per reactor year, and "extremely unlikely events" with frequencies between $10^{-4}$ and $10^{-6}$ per reactor-year. Events with frequencies below $10^{-6}$ per reactor-year were classified as "beyond design basis events".

2. Within the design basis event envelope, events of greater severity shall have lower frequency."

NRCs Pre-application Safety Evaluation Report (PSER) of PRISM Liquid-Metal Reactor and the supporting PRA is documented in Reference [43] completed in 1994. The PRISM conceptual design was submitted by the U.S. Department of Energy (DOE) in accordance with the NRC's "Statement of Policy for the Regulation of Advanced Nuclear Power Plants" (51 Federal Register 24643). This policy provides for the early Commission review and interaction with designers and licensees. The PRISM reactor design proposed by DOE is for a small, modular, pool-type, liquid-metal (sodium)-cooled reactor.

The approach followed in the selection of LBEs to support this review is captured in the following excerpt from the PSER:

"The methodology used by General Electric (GE) for defining the design-basis events (DBEs) for the PRISM reactor is described in Chapter 15 of the PSID. The procedure is systematic and draws upon PRA work performed in the conceptual stage of the design. The PRA is used to help ensure completeness in the identification of accident sequences and to rank the sequences in order of their importance on the basis of their expected occurrence frequency and offsite consequences. Each event is placed into a category of either a DBE or a beyond-design-basis event (BDBE). GE has considered all events occurring at a frequency of $10^{-6}$ or more per reactor-year to be DBEs. GE analyzes these events in a conservative manner. Less likely events are considered BDBEs (frequencies $<10^{-6}$ per reactor-year). GE considers these off-normal conditions of such extremely low probability that no event in this category is considered credible during the plant's lifetime. BDBEs can, however, have significant consequences. GE acknowledges some of these events may merit consideration in establishing the design. These BDBEs are discussed in Appendices E and G of the PSID."

In its review, the NRC staff used a somewhat different definition of design basis event categories than proposed by GE based on expected frequency of occurrence.

In addition to reviewing the results of the PRISM PRA for the purpose of event identification, the NRC staff defined a set of postulated "Bounding Events" that were added to Category EC-III to support the safety evaluation. The methodology used to define these bounding events was not provided so it appears to be based on the staff's engineering judgment. Subjective estimates of the frequency of occurrence of these bounding events were provided which included estimates as low as $10^{-10}$ per reactor year, including one extreme event involving removal of all control rods with failure to scram, and station blackout events 
lasting as long as 36 hours. There are no comparably extreme events considered in licensing currently operating LWR plants.

The PRISM PRA that was reviewed was performed for a single reactor module, so the question of multimodule accidents did not come up in the NRC review. However, more recently GE-Hitachi has completed an upgrade to the PRISM PRA to pilot the ASME/ANS PRA standard for advanced nonLWRs $^{[32]}$ and multi-module accidents were addressed in the more recent work. More information on the PRISM PRA is provided in a companion report on PRA development to support this LMP.

\section{A.1.3.5 ANS DESIGN STANDARD FOR MODULAR HELIUM COOLED REACTOR PLANTS ${ }^{[25]}$}

This standard, ANS 53.1 "Modular Helium Reactor Safety Design" was issued in 2011 to provide a riskinformed and performance-based design process for modular helium cooled reactors (MHRs). The purpose of this standard is to provide nuclear safety criteria applicable to the design of MHRs leading up to the preparation of a license. To achieve this purpose, this standard provides a process that can be used to:

- $\quad$ Develop MHR top-level nuclear regulatory safety criteria

- Identify safety functions, top-level design criteria, licensing-basis events, design basis accidents, and methods for performing safety analyses

- $\quad$ Determine safety classification of SSCs

- Identify safety-related SSC special treatment requirements and defense-in-depth (DID) provisions

- $\quad$ Demonstrate the adequacy of DID by applying a risk-informed evaluation approach

The standard was influenced by the RIPB design and licensing approach employed for the MHTGR and subsequently refined in the Exelon, PBMR and NGNP white papers. The flow chart used to describe the process advanced in this standard shown in Figure A-2 is essentially the same as a flow chart found in the NGNP Defense-in-Depth Approach white paper. ${ }^{[26]}$ An important contribution made in this standard is specific guidance for the designer to implement the processes described at a high level in the NGNP white papers on LBE selection, PRA development, SSC safety classification, mechanistic source term development, and evaluation of DID adequacy. With influence from that same white paper, this standard included specific design criteria for evaluating the adequacy of defense-in-depth. These criteria are derived from the DID principles listed in Table A-3 and provide examples of DID principles to evaluate the selection of LBEs as discussed more fully in Section 4. 


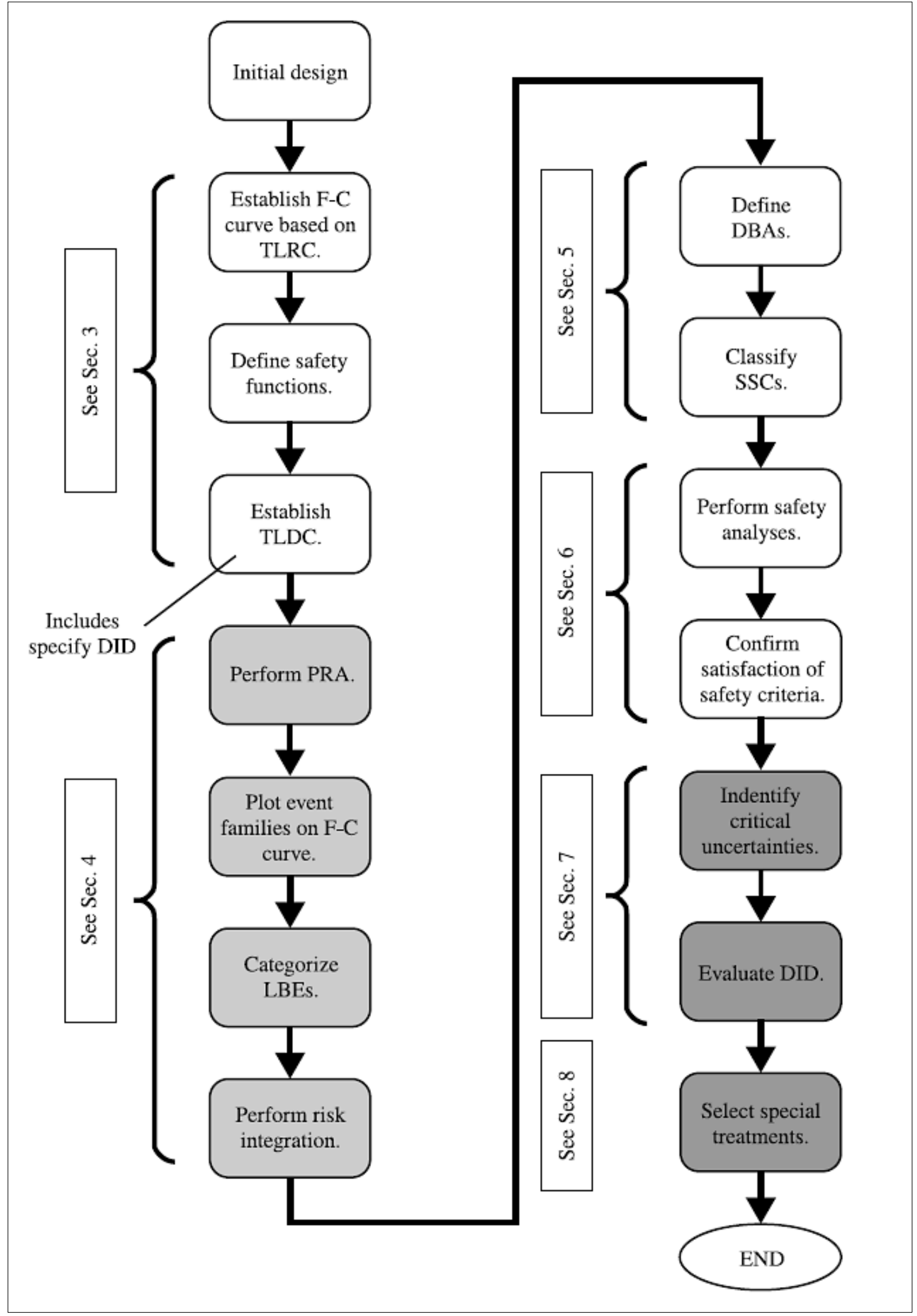

Figure A-2. MHR Safety Design Process in ANS 53.1 
Table A-3. Defense-in-Depth Principles from ANS 53.1

\begin{tabular}{|c|c|}
\hline 1 & Radionuclide release barriers are sufficiently robust to withstand challenges identified for the design. \\
\hline 2 & Each barrier's failure probability is acceptably low compared with identified challenges. \\
\hline 3 & $\begin{array}{l}\text { As-designed, built, and maintained multiple radionuclide release barriers minimize dependencies. Events } \\
\text { that challenge two or more barriers are infrequent, and the postulated failure of one barrier does not } \\
\text { significantly increase the failure probability of another barrier. }\end{array}$ \\
\hline 4 & Overall barrier redundancy and diversity ensure compatibility with the TLSC. \\
\hline 5 & $\begin{array}{l}\text { Accidents potentially releasing significant radioactive material quantities preserve a reasonable } \\
\text { prevention/mitigation balance. }\end{array}$ \\
\hline 6 & Safety design avoids overreliance on programs to compensate for plant design weaknesses. \\
\hline 7 & $\begin{array}{l}\text { System redundancy, independence, and diversity cover expected challenges based on frequency, system } \\
\text { failure consequences, and associated uncertainties. }\end{array}$ \\
\hline 8 & The safety design adequately addresses common-cause failures. \\
\hline 9 & $\begin{array}{l}\text { Performance of a risk-significant safety function is not reliant on a single engineered feature except where } \\
\text { inherent safety is demonstrated for all failure modes. }\end{array}$ \\
\hline 10 & $\begin{array}{l}\text { The approach evaluates human-error likelihood and consequences, thus providing defenses against human } \\
\text { errors that can lead to significant radioactive material release. }\end{array}$ \\
\hline 11 & $\begin{array}{l}\text { The design meets the GDC intent applicable in } 10 \text { CFR 50, Appendix A, and reactor-specific regulatory } \\
\text { design criteria from RI-PB licensing. }\end{array}$ \\
\hline
\end{tabular}

\section{A.1.3.6 YUCCA MOUNTAIN PRE-CLOSURE SAFETY ANALYSIS (PCSA) ${ }^{[27]}$}

Additional guidance for selecting LBEs was found in the case of the Yucca Mountain Pre-Closure Safety analysis which was submitted and successfully reviewed by the NRC staff. ${ }^{[28]}$ It is relevant to this project because it is an example of a safety and regulatory evaluation of a first-of-a-kind nuclear facility, it utilized a risk-informed process to inform the design and to select LBEs, made use of frequency and dose criteria tied to the regulations, and exhibits key elements of a technically sound and well structured, riskinformed and performance-based design making process.

Congress established the bases for NRC licensing of a geologic high level nuclear waste (HLW) repository at Yucca Mountain. NRC was authorized to exercise its licensing and regulatory authority under the Atomic Energy Act of 1954, as amended (42 U.S.C. 2011 et seq.), to license and regulate a DOE facility for the disposal of HLW, including spent nuclear fuel (SNF). Subsequently, Congress authorized the $\mathrm{NRC}$ to promulgate technical requirements and criteria that it would apply to determine whether to approve or disapprove of DOE applications to construct a repository, receive, and possess HLW and SNF in a repository, and close and decommission a repository.

In 2001, the NRC issued its technical requirements and criteria in 10 CFR 63. ${ }^{[29]}$ In 2003, the NRC issued NUREG-1804, Rev 2, The Yucca Mountain Review Plan. ${ }^{[30]}$ This plan provides guidance for the NRC staff to evaluate a U.S. Department of Energy license application for a geologic repository. Unlike licensing associated with 10 CFR 50, regulatory guides were not associated with 10 CFR 63.

The regulation and the review plan address the pre-closure period of operation in which waste is emplaced into Yucca Mountain and the period thereafter in which the permanent closure and permanent storage within Yucca Mountain occurs. DOE is the licensee and established nominally 100 years as the 
pre-closure period for purposes of safety analysis. This section discusses only the safety analysis and interaction with the design and engineering associated with the pre-closure period.

According to the 10 CFR 63.2 definition, an initiating event means a natural or human-induced event that causes an event sequence. Consistent with this definition, an initiating event is a departure from normal operation that triggers an event sequence. These definitions are consistent with those used in nuclear reactor PRAs. ${ }^{[31]}$ As defined in 10 CFR 63.2, event sequence means a series of actions or occurrences or both within the natural and engineered components of a geologic repository operations area (GROA) that could potentially lead to exposure of individuals to radiation.

Combining the 10 CFR 63.2, 10 CFR 63.111 and 10 CFR 63.204 definitions, important to safety (ITS), with reference to structures, systems, and components, means those engineered features of the geologic repository operations area whose function is:

1. "To provide reasonable assurance that high-level waste can be received, handled, packaged, stored, emplaced, and retrieved such that no member of the public in the general environment receives more than an annual dose of $0.15 \mathrm{mSv}$ (15 mrem) during normal operation and Category 1 event sequences,

2. To prevent or mitigate each Category 2 event sequence that could result in radiological exposures to any individual at or beyond the site boundary, that could result in the more limiting of a TEDE of $0.05 \mathrm{~Sv}$ (5 rem), or the sum of the deep dose equivalent and the committed dose equivalent to any individual organ or tissue (other than the lens of the eye) of $0.5 \mathrm{~Sv}(50 \mathrm{rem})$. The lens dose equivalent may not exceed $0.15 \mathrm{~Sv}(15 \mathrm{rem})$, and the shallow dose equivalent to skin may not exceed $0.5 \mathrm{~Sv}$ (50 rem)."

Table A-4 presents the performance criteria that were developed. Category 1 event sequences are those that could occur at least once over the pre-closure period which is equivalent to a frequency at least $10^{-2}$ per year. Category 2 event sequences are those that could occur with frequency less than $10^{-2}$ per year but greater than or equal to a frequency of $10^{-6}$ per year. Less frequent event sequences are termed "Beyond Category 2." Performance objectives were not specified for Beyond Category 2 event sequences. There are no NRC equivalents to QHOs defined for such events for Yucca Mountain. 
Table A-4. Performance Criteria for Category 1 and 2 Event Sequences and Normal Operation

\begin{tabular}{|c|c|c|c|c|c|}
\hline Event Sequence Type & $\begin{array}{l}\text { Category of } \\
\text { Individual }\end{array}$ & $\begin{array}{c}\text { GROA }^{\mathrm{a}} \\
\text { Restricted Areas }\end{array}$ & $\begin{array}{c}\text { Site } \\
\text { (Preclosure } \\
\text { Controlled Area) }\end{array}$ & $\begin{array}{c}\text { Offsite }^{\mathrm{b}} \text { in the } \\
\text { General Environment } \\
\text { (Unrestricted Area) }\end{array}$ & $\begin{array}{c}\text { Offsite }{ }^{\mathrm{b}} \text {, but not } \\
\text { within the General } \\
\text { Environment } \\
\text { (Unrestricted Area) }\end{array}$ \\
\hline \multirow{2}{*}{$\begin{array}{l}\text { Aggregate of Normal Operation and Category } 1 \text { Event } \\
\text { Sequences Dose } \\
\text { (Category 1-Those event sequences that are } \\
\text { expected to occur one or more times before } \\
\text { permanent closure of the GROA) }\end{array}$} & Public & - & 100 mrem/yr d,e,f,g & $\begin{array}{l}15 \mathrm{mrem} / \mathrm{yr}^{\mathrm{h}, \mathrm{i}} \\
2 \mathrm{mrem} \text { in any hour }{ }^{9}\end{array}$ & $\begin{array}{l}100 \mathrm{mrem} / \mathrm{yr}^{\mathrm{d}, \mathrm{e}, \mathrm{f}} \\
2 \mathrm{mrem} \text { in any hour }\end{array}$ \\
\hline & $\begin{array}{l}\text { Radiation } \\
\text { worker }^{k, l}\end{array}$ & $\begin{array}{l}5 \mathrm{rem} / \mathrm{yr} \text { d,e,m } \\
50 \mathrm{rem} \text { to any organ } \\
15 \mathrm{rem} \text { lens of eye } \\
50 \text { rem skin }\end{array}$ & See note $n$. & See note $\mathrm{n}$. & See note $n$. \\
\hline $\begin{array}{l}\text { Single Category } 2 \text { Event Sequence Dose } \\
\text { (Category } 2 \text {-Other event sequences that have at } \\
\text { least one chance in } 10,000 \text { of occurring before } \\
\text { permanent closure of the GROA) }\end{array}$ & Public & - & - & $\begin{array}{l}5 \text { rem }^{\circ} \\
50 \text { rem to any organ } \\
15 \text { rem lens of eye } \\
50 \text { rem skin }\end{array}$ & $\begin{array}{l}5 \text { rem }^{\circ} \\
50 \text { rem to any organ } \\
15 \text { rem lens of eye } \\
50 \text { rem skin }\end{array}$ \\
\hline
\end{tabular}

NOTE: ${ }^{a}$ Other areas of the site may be identified as restricted areas as required by operations.

bOffsite areas are areas outside of the preclosure controlled area (See Figure 1.8-2).

c10 CFR 63.2.

d10 CFR 63.111(a)(1).

e 10 CFR $63.111(\mathrm{~b})(1)$

f10 CFR 20.1301(a)(1).

910 CFR 20.1301(a)(2)(b)

h10 CFR 63.111(a)(2).

10 CFR 63.204.

j10 CFR 20.1301(a)(2).

kIndividual with assigned duties involving exposure to radiation or to radioactive material.

'Occupational doses are those received during the course of those assigned duties.

m10 CFR 20.1201.

nIf receiving an occupational dose (see note $k$ above) at this location, the GROA restricted areas' occupational objectives apply; otherwise, the individual is considered a member of the public.

10 CFR 63.111(b)(2).

10 CFR 63.111 further states that a pre-closure safety analysis (PCSA) must be performed and

10 CFR 63.112 specifies the scope of that analysis. As the licensee, the DOE elected to use a

combination of probabilistic risk assessment methods, hazard analysis methods and deterministic methods as the basis for the PCSA. The analysis demonstrated compliance with the performance objectives for internal events, on-site hazards, off-site hazards, and natural phenomena hazards. DOE submitted a license application under this regulation in June 2008. Requests for Additional Information were made and answered during 2009 and 2010. The NRC's five-volume Safety Evaluation Report was issued over the period 2010 to 2015 with a positive assessment of the PCSA. During the development of the license application submitted in 2008, the design and PCSA proceeded concurrently such that engineering and design were heavily influenced by the PCSA and visa-versa. Figure A-3 is an overview of the riskmanaged design process emphasizing the pre-closure safety analysis steps. 


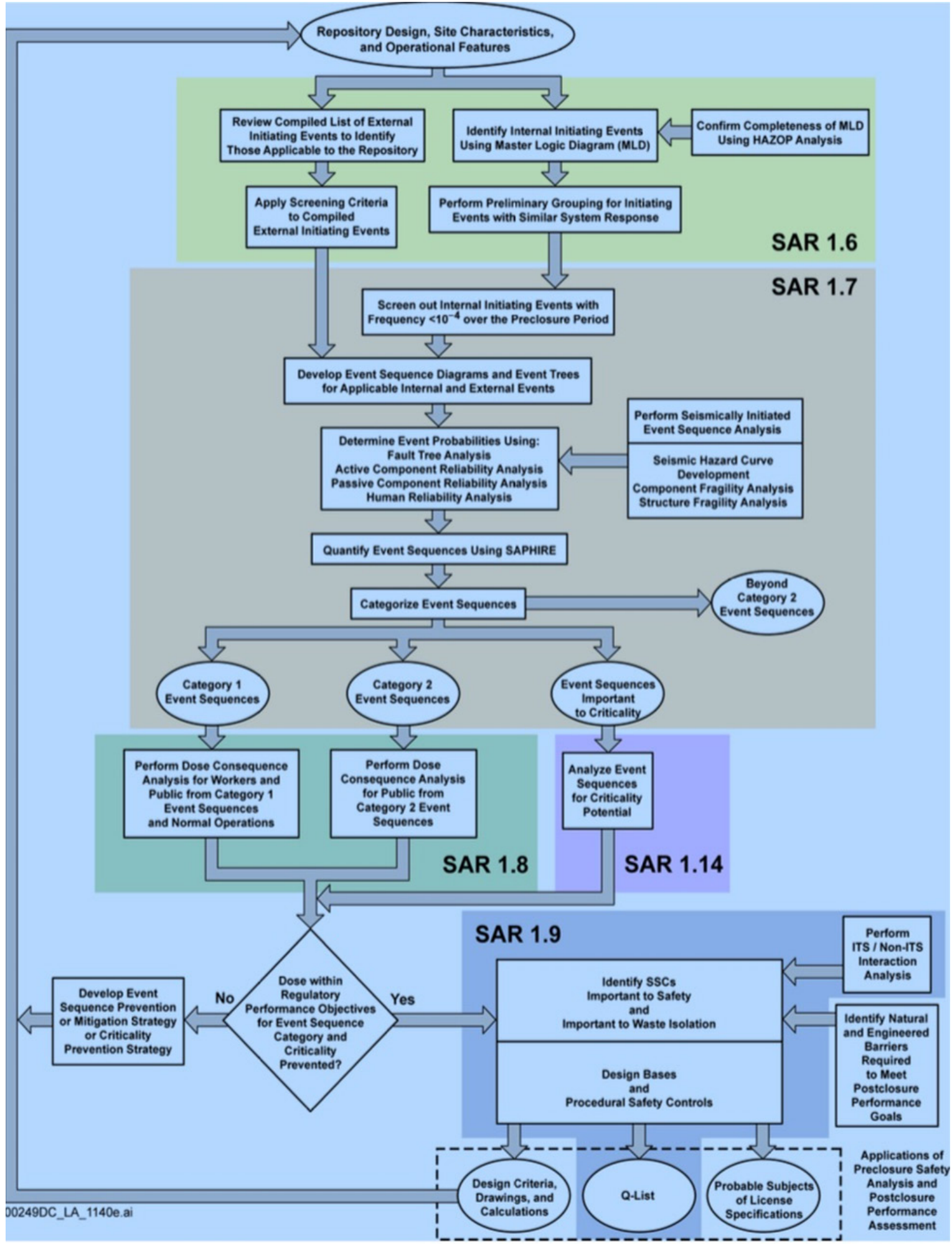

Figure A-3. Use of the PCSA for Risk Management of Repository Design

The PCSA, as is typical of a high-quality PRA, may be thought of as a simulation of how a facility with its systems and personnel acts and reacts when something goes wrong (i.e. in response to an initiating 
event). The PCSA identified design bases and procedural safety controls for ITS SSCs that prevent (i.e., reduce the likelihood of) or mitigate (i.e., reduce the severity of) event sequences. The PCSA also provided inputs for developing license specifications as well as management, maintenance, training, and operations programs that ensure the availability of ITS SSCs. The PCSA was a collaborative effort with repository design groups. Preliminary event sequences were identified early in the design, and safeguards were incorporated into the design to reduce event sequence probabilities, including those that involved human error as well as hardware. As a practical matter, the safety analysis staff reviewed every aspect of the design relevant to the nuclear safety model (e.g., the event sequences and hazard analyses). As more detail was added to the design, more detail was added to the models. When a performance objective was in jeopardy, the safety analysis staff defined what needed to be changed either to mitigate or prevent the event sequence or hazard. It was then the responsibility of the engineering and design staff to modify the design criteria and specifications to achieve the nuclear safety objectives. The PCSA, therefore, was an integral part of the design process.

Design, site, and operational information from various disciplines were inputs to the PCSA. Design information used to identify the initiating events and to conduct the event sequence analyses was obtained from design documents, such as design drawings, design reports, piping and instrumentation diagrams, control logic diagrams, and design calculations. Design information on locations and amounts of radioactive material present was used in performing consequence and criticality analyses. Site information, such as wind patterns, proximity of potentially hazardous materials, and seismicity, was also used in the PCSA particularly for natural and man-made on-site and off-site hazard analyses. Representative waste containers, rather than those of specific vendors, were analyzed for their failure potential associated with event sequences. A range of radioactive HLW and SNF container dimensions and materials and internal configurations were considered within these representative analyses.

The experience with the PCSA at Yucca Mountain was found to be very useful in defining the approach to selecting LBEs described in Section 4 including the use of frequency and dose criteria to evaluate the risk of LBEs. The fact that a PRA approach was used to meet safety and licensing requirements for a new facility that lacked any relevant deterministic regulatory precedents is significant. In addition, the NRC regulations for this new facility included specific numerical criteria for the frequencies and doses of LBEs that were used to establish the original licensing basis. Finally, the Yucca Mountain Pre-Closure Facility licensing approach included the development of performance requirements for SSCs in the prevention and mitigation of accidents. This work was also found to be useful in develop the PRA approach for LBE selection, the RIPB approaches to SSC safety classification and DID that are discussed more fully in companion reports.

\section{A.1.4 REGULATORY FOUNDATION FOR ESTABLISHING RIPB FREQUENCY-CONSEQUENCE TARGETS}

The focus of this section is to define criteria that establish evaluation criteria on the frequencies or consequences of LBEs and LBE categories that must be considered in the design and operation of a nuclear power plant in order to assure public safety and to assess the adequacy of the performance of SSCs that perform safety functions during these LBEs. F-C Targets serve as evaluation criteria to evaluate the risk significance of LBEs in terms of their frequencies and consequences. This evaluation is performed in order to assure public safety and to assess the adequacy of the performance of SSCs that perform safety functions during these LBEs. The F-C Targets are based on the following objectives:

1. Provide direct public health and safety acceptability evaluation boundaries in terms of individual radiological consequences, i.e., performance-based criteria 
2. Are independent of reactor type and site

3. Provide well-defined, quantifiable risk criteria

The following primary sources have been identified as containing criteria or concepts that can be used to establish evaluation boundaries on the risk or consequences of potential radiological releases from nuclear power plants in the United States. Each was considered in this report. Not all are equivalent or individually additive, thus requiring some judgment in synthesizing the F-C Targets for the LMP.

- Reactor Safety Goal Policy Statement: ${ }^{[11]}$ On August 4, 1986, the NRC adopted a safety goal policy for the operation of nuclear power reactors. The objective of this policy is to establish goals that broadly define an acceptable level of radiological risk. Two qualitative safety goals supported by two Quantitative Health Objectives were established. These two supporting objectives are based on the principle that nuclear risks should not be a significant addition to other societal risks.

This policy limits public safety risk resulting from nuclear power plant operation. Limits are stated in the form of the maximum allowable risk of immediate death and the risk of delayed mortality from exposure to radiological releases of all types from nuclear power plants.

- 10 CFR 20, "Standards for Protection against Radiation (Subpart C, Occupational Dose Limits)": ${ }^{[52]}$ The regulations promulgated under 10 CFR 20 establish standards for protection against ionizing radiation resulting from activities conducted under licenses issued by the NRC. Event sequences expected to occur within the plant lifetime, considering multiple reactor modules, are classified as AOOs. AOOs are evaluated against the dose limits derived from annual dose limits in 10 CFR 20.

- 10 CFR 20, "Standards for Protection against Radiation (Subpart D, Radiation Dose Limits for Individual Members of the Public)": [53] These criteria (\$20.1301) specify annual dose limits for releases associated with relatively high frequency events that occur as part of normal plant operations.

- 10 CFR 50, Appendix I, "Numerical Guides for Design Objectives and Limiting Conditions for Operation to Meet the Criterion 'As Low as is Reasonably Achievable' for Radioactive Material in Light-Water-Cooled Nuclear Power Reactor Effluents": ${ }^{[54]}$ This appendix provides explicit annual limits on doses from planned discharges that meet the NRC's definition of ALARA.

- 10 CFR 52, Subpart C, “Combined Licenses": ${ }^{[55]}$ Under the provisions of 10 CFR 52.79, ${ }^{[56]}$ an application for a combined license must include the principal design criteria for a proposed facility. The principal design criteria establish the necessary design, fabrication, construction, testing, and performance requirements for SSCs important to safety; that is, SSCs that provide reasonable assurance that the facility can be operated without undue risk to the health and safety of the public. This standard repeats the same dose requirements as specified in 10 CFR 100 and 10 CFR 50.34(a)(ii)(d).

- 40 CFR 190, “Environmental Radiation Protection Standards for Nuclear Power Operations": ${ }^{[57]}$ These standards provide the generally applicable exposure limits for members of the general public from all operations except transportation and disposal or storage of spent fuel associated with the generation of electrical power by nuclear power plants.

- 10 CFR 100, "Reactor Site Criteria (Subpart B, Evaluation Factors for Stationary Power Reactor Site Applications on or After January 10, 1997)": ${ }^{[58]} § 100.20$ defines the EAB and low population zones (LPZs) of a nuclear reactor site, and requires that the combination of the site and reactor located on that site be capable of meeting the dose and dose rate limitations set forth in 10 CFR $\S 50.34(a)$. 
- 10 CFR §50.34(a)(ii)(d), “Contents of Applications: Technical Information": ${ }^{[59]}$ This section of the regulation specifies dose limits for evaluating the acceptance of the engineered safety features that are intended to mitigate the radiological consequences of accidents. These dose limits are consistent with those utilized in 10 CFR 100 for determining the extent of the EAB and Emergency Planning Zone (EPZ).

- NUREG-0800, Standard Review Plan Chapter 15.0 Introduction - Transient and Accident Analyses: ${ }^{[3]}$ This document specifies acceptance criteria for AOOs and states the principle that the risks of AOOs and postulated accidents as defined by the product of the frequency and consequence should be about the same. The acceptance criteria permit the doses from lower frequency AOOs to be greater than the annual dose limits in 10 CFR 20 as long as other acceptance criteria, including the need to avoid restrictions on uncontrolled areas are met.

- NUREG-1860 Frequency-Dose Criteria: ${ }^{[21]}$ This document, reviewed in the previous section, proposes an approach for identifying and classifying licensing basis events into frequency categories, and frequency-dose criteria for evaluating the risks of radiological exposures.

- United Kingdom Safety Assessment Principles: ${ }^{[39]}$ The UK SAPs, reviewed in the previous section, provide frequency-consequence criteria for evaluation of risks at the reactor level and at the integrated site level.

Each of these primary sources is discussed in greater detail below and their use in the selection of frequency-consequence evaluation criteria in Section 3. The U.S. regulations have been grouped into three sets of criteria, consistent with the category of event(s) to which they apply. The U.K. SAPs, which span the full spectrum of LBEs are discussed in a separate section.

\section{A.1.4.1 F-C TARGETS RELATED TO NORMAL OPERATION AND AOOS}

10 CFR 50.34, 10 CFR 20, and Appendix I of 10 CFR 50 all provide guidance on the limits for radiological releases from reactors during normal operations.

The regulations do not define the term "normal operation" in quantitative terms, i.e., the expected frequency of specified anticipated occurrences. However, Appendix A to 10 CFR 50 defines AOOs as "those conditions of normal operation... expected to occur one or more times during the life of a nuclear power plant."

NUREG-0800 SRP Chapter 15.0 specifies acceptance criteria for AOOs and states the principle that the risks of AOOs and postulated accidents as defined by the product of the frequency and consequence should be the same. The acceptance criteria permit the doses from a specific AOO to be greater than the annual dose limits in 10 CFR 20 as long as other acceptance criteria are met. The following quotes from NUREG-0800 Chapter 15.0 elaborate on these points:

"If the risk of an event is defined as the product of the event's frequency of occurrence and its consequences, then the design of the plant should be such that all the AOOs and postulated accidents produce about the same level of risk (i.e., the risk is approximately constant across the spectrum of AOOs and postulated accidents). This is reflected in the general design criteria $(G D C)$, which generally prohibit relatively frequent events (AOOs) from resulting in serious consequences, but allow the relatively rare events (postulated accidents) to produce more severe consequences."

In specifying the F-C Targets for the least likely AOO events, referred to by the ANS classification "Condition III" events, the following criterion is provided: 
"For PWRs, the release of radioactive material may exceed guidelines of 10 CFR Part 20, but shall not be sufficient to interrupt or restrict public use of those areas beyond the exclusion radius.

For BWRs, the offsite release of radioactive material is limited to a small fraction of the guidelines of 10 CFR Part 100, which may be the result of the failure of a small fraction ${ }^{*}$ of the fuel elements in the reactor."

The combination of these statements supports the view that the annual dose limits in 10 CFR 20 should be interpreted as annual risk limits and not limits to be applied to individual AOO events of low frequency. For the least likely (Condition III) events in the AOO category, these acceptance criteria clearly suggest that dose per event limits can be proportionally greater as long as the annual risk limit is not exceeded. This insight is used to revisit the derivation of the F-C Targets that were developed in the NGNP LBE white paper ${ }^{[1]}$, as discussed more fully in Section 4.

10 CFR $\$ 20.1301$ requires that the TEDE for a member of the public be limited to 100 mrem "from licensed operation... in a year" and 2 mrem in any 1 hour, in unrestricted areas. Presumably, unrestricted area means off-site and at the site boundary. This regulation provides the applicable criteria for limiting dose to the general public from anticipated and unanticipated events associated with the normal (nonaccident) operation of a nuclear power plant.

10 CFR 50, Appendix I, identifies dose and dose rate limits and limits on planned releases from the operation of nuclear power plant rad-waste systems during normal operation, to maintain exposures ALARA. These criteria provide implementation guidance for applying the requirements of 10 CFR 50.34(a) and 10 CFR 50.36(a), for planned releases from the radwaste systems of nuclear power plants to the general environment to be ALARA. These requirements specify the following limits:

"The applicant shall provide reasonable assurance that the following design objectives will be met.

A. The calculated annual total quantity of all radioactive material above background to be released from each light-water-cooled nuclear power reactor to unrestricted areas will not result in an estimated annual dose or dose commitment from liquid effluents for any individual in an unrestricted area from all pathways of exposure in excess of 3 millirems to the total body or 10 millirems to any organ.

B.1. The calculated annual total quantity of all radioactive material above background to be released from each light-water-cooled nuclear power reactor to the atmosphere will not result in an estimated annual air dose from gaseous effluents at any location near ground level which could be occupied by individuals in unrestricted areas in excess of 10 millirads for gamma radiation or 20 millirads for beta radiation."

\section{A.1.4.2 F-C TARGETS RELATED TO DBES}

10 CFR $\$ 50.34(a)(1)$ contains NRC's regulations governing the design of new reactors and the means provided to protect against DBAs. This LBE selection approach uses the term "events" in lieu of

\footnotetext{
*Although the term "small fraction" is not quantified in this part of the SRP, in Chapter 15.0.3 the term "small fraction" of another dose limit is defined as $10 \%$ of the referenced dose limit.
} 
"accidents" (as found in the regulations discussed below) for the identification of unplanned, off-normal events not expected in the plant lifetime.

10 CFR $§ 50.34(a)(1)$ requires that any reactor be designed such that:

"An individual located at any point on the EAB would not receive a radiation dose in excess of 25 rem TEDE for any 2-hour period following the onset of a postulated fission product release. An individual located at any point on the outer boundary of the LPZ, exposed to the radioactive cloud resulting from a postulated fission product release, would not receive a radiation dose for any 30-day period in excess of 25 rem TEDE."

10 CFR $\S 50.34(a)(i i)(D)$ requires that these consequence limits be used when evaluating the acceptability of the features included in the plant design (i.e., engineered safety features and fission product barriers) for mitigating accident radioactive releases. The footnote pertaining to this section states that the fission product release to be assumed should be based "upon a major accident... postulated from consideration of possible accidental events." 10 CFR $§ 100.21(c)(2)$, "Reactor Site Criteria: non-seismic site criteria," requires that the radiological dose consequences of postulated accidents meet the criteria stated in 10 CFR $\S 50.34(\mathrm{a})(1)$ for the type of facility located at the site in question.

In general, NRC's regulations do not define the type of events that comprise the category of DBAs. For LWRs, the GDC (Appendix A to 10 CFR 50) indicates that LOCAs must be considered as postulated accidents when designing safety systems. However, the Standard Review Plan $§ 15.0$ includes a list of generic events appropriate for LWRs some of which may be applicable to other reactor types. It is stated in the SRP that the applicant is expected to define the appropriate "limiting" events for its design and sufficient design information for the staff to review the event selection. However, there is no method specified or referenced for ensuring that the appropriate set of "limiting" design specific events have been identified.

The regulations do not define DBAs in terms of their expected frequencies of occurrence, but 10 CFR $\$ 50.34(a)(i)(2)$ articulates the expectation that the design, construction, and operation of nuclear power reactors will be such as to produce an "extremely low probability of occurrence" for accidents that could release significant quantities of radioactive fission products. No quantitative definition of the term "extremely low probability" is provided in the regulation.

In the licensing of advanced non-LWRs, the NRC has introduced review acceptance criteria that clarifies the association of the 10 CFR $\$ 50.34$ (a)(i)(2) dose thresholds with the extremely unlikely large LOCA DBA, but expects that more likely DBAs be held to more stringent dose thresholds. Table A-5 lists different events with different dose thresholds: ${ }^{[63]}$ 
Table A-5. Accident-Dose Criteria (Table 1 from Reference [63])

\begin{tabular}{|c|c|c|}
\hline Accident or Case & $\begin{array}{l}\text { EAB and LPZ } \\
\text { Dose Criteria } \\
25 \text { rem TEDE }\end{array}$ & $\begin{array}{c}\text { Analysis Release Duration } \\
30 \text { days for all leakage pathways }\end{array}$ \\
\hline $\begin{array}{l}\text { BWR Main Steam Line Break } \\
\text { Fuel Damage or Pre-incident Spike } \\
\text { Equilibrium lodine Activity }\end{array}$ & $\begin{array}{l}25 \text { rem TEDE } \\
2.5 \text { rem TEDE }\end{array}$ & $\begin{array}{l}\text { Instantaneous puff, until MSIV } \\
\text { isolation }\end{array}$ \\
\hline BWR Rod Drop Accident & 6.3 rem TEDE & 24 hours \\
\hline Small Line Break Accident & 2.5 rem TEDE & $\begin{array}{l}\text { Until isolation, if capable, or until } \\
\text { cold shutdown is established }\end{array}$ \\
\hline $\begin{array}{l}\text { PWR Steam Generator Tube Rupture } \\
\text { Fuel Damage or Pre-incident Spike } \\
\text { Coincident lodine Spike } \\
\end{array}$ & $\begin{array}{l}25 \text { rem TEDE } \\
2.5 \text { rem TEDE }\end{array}$ & $\begin{array}{l}\text { Affected SG: time to isolate; } \\
\text { Unaffected SG(s): until cold } \\
\text { shutdown is established }\end{array}$ \\
\hline $\begin{array}{l}\text { PWR Main Steam Line Break } \\
\text { Fuel Damage or Pre-incident Spike } \\
\text { Coincident lodine Spike } \\
\end{array}$ & $\begin{array}{l}25 \text { rem TEDE } \\
2.5 \text { rem TEDE }\end{array}$ & Until cold shutdown is established \\
\hline PWR Locked Rotor Accident & 2.5 rem TEDE & Until cold shutdown is established \\
\hline PWR Rod Ejection Accident & 6.3 rem TEDE & $\begin{array}{l}30 \text { days for containment leakage } \\
\text { pathway; Until cold shutdown is } \\
\text { established for secondary } \\
\text { pathway }\end{array}$ \\
\hline Fuel Handling Accident or Cask Drop & 6.3 rem TEDE & 2 hours \\
\hline
\end{tabular}

The explanation for assigning different dose criteria given for this table is as follows:

"The dose acceptance criteria in Table 1(Table 5 below) of this SRP section are fractions of the 10 CFR 50.34(a)(1) dose reference values for accidents other than the LOCA, as has been done historically. For events having a moderate frequency of occurrence, any release of radioactive material must be such that the calculated offsite doses are a small fraction of the 10 CFR 50.34(a)(1) reference values. A small fraction is defined as less than $10 \%$ of the 10 CFR 50.34(a)(1) reference values, or 2.5 rem TEDE. The plant site and dose mitigating engineered safety features are acceptable with respect to the radiological consequences of a postulated control rod drop accident (BWR), control rod ejection accident (PWR), fuel handling accident or cask drop accident if the calculated offsite doses are well within the dose reference values in 10 CFR 50.34(a)(1). 'Well within' is defined as 25\% of the 10 CFR 50.34(a)(1) reference values, or 6.3 rem TEDE."

The assumptions made by the staff in associating events with either a $25 \%$ dose threshold or $10 \%$ dose threshold to a numerical frequency of occurrence are not specified. A search of the supplied references was not successful in establishing the staff's estimates of the event frequencies or other basis for using these more limiting dose thresholds for DBAs more likely than the limiting event. As a result, this information is not directly used in developing frequency-consequence criteria. However, the idea that the acceptable doses should be linked to the frequency of occurrence of any event is utilized in selecting the LMP F-C Targets. 


\section{A.1.4.3 F-C TARGETS RELATED TO POLICY GUIDANCE FOR BDBES}

Current policy and guidance require that certain events outside the scope of the normal operation and DBE categories be considered in the design of nuclear power plants.

The NRC's "Policy Statement on Severe Reactor Accidents Regarding Future Designs and Existing Plants" states the Commission's intent to "...take all reasonable steps to reduce the chances of occurrence of a severe accident involving substantial damage to the reactor core and to mitigate the consequences of such an accident should one occur." As noted earlier, this policy statement specifically addresses the Commission's intent to resolve safety issues associated with "accidents more severe than design basis accidents." This policy statement provides the following criteria for evaluating new designs for safety adequacy for addressing severe accident potential.

"The Commission believes that a new design for a nuclear power plant (as well as a proposed custom plant) can be shown to be acceptable for severe accident concerns if it meets the following criteria and procedural requirements:

a. Demonstration of compliance with the procedural requirements and criteria of the current Commission regulations, including the Three Mile Island requirements for new plants as reflected in the CP Rule (10 CFR 50.34(f)):

b. Demonstration of technical resolution of applicable unresolved Safety Issues, including a special focus on assuring the reliability of decay heat removal systems and the reliability of both AC and DC electrical supply systems:

c. Completion of a Probabilistic Risk Assessment (PRA) and consideration of the severe accident vulnerabilities the PRA exposes along with the insights that it may add to the assurance of no undue risk to public health and safety:

d. Completion of a staff review of the design with a conclusion of safety acceptability using an approach that stresses deterministic engineering analysis and judgment complemented by PRA."

In addition to its Severe Accident Policy, the Commission has issued NUREG-0880, "Safety Goals for Nuclear Power Plant Operation" and the related policy statement entitled "Safety Goals for the Operation of Nuclear Power Plants."[11] Two qualitative safety goals are used to express the Commission's policy regarding the acceptable level of radiological risk from nuclear power plant operation as follows:

- "Individual members of the public should be provided a level of protection from the consequences of nuclear power plant operation such that individuals bear no significant additional risk to life and health.

- Societal risks to life and health from nuclear power plant operation should be comparable to or less than the risks of generating electricity by viable competing technologies and should not be a significant addition to other societal risks."

The following QHOs were identified as the basis for determining achievement of the above safety goals:

"The risk to an average individual in the vicinity of a nuclear power plant of prompt fatalities that might result from reactor accidents should not exceed one-tenth of one percent (0.1 percent) of the sum of prompt fatality risks resulting from other accidents to which members of the U.S. population are generally exposed. 
The risk to the population in the area near a nuclear power plant of cancer fatalities that might result from nuclear power plant operation should not exceed one-tenth of one percent (0.1 percent) of the sum of cancer fatality risks resulting from all other causes."

The statement of risks provided in the Safety Goal Policy envelops the spectrum of allowable risk associated with the operation of a nuclear power plant. As such, it clearly defines the outermost boundaries of acceptable risk associated with any event that has the potential to produce a radiological release affecting the environment or the health and safety of the general public.

\section{A.1.4.4 CRITERIA FOR CLASSIFYING LBES BASED ON FREQUENCY OF OCCURRENCE}

In its June 26, 1990, SRM on SECY-90-16, ${ }^{[35]}$ the Commission endorsed a core damage frequency (CDF) goal of $10^{-4}$ per year for advanced reactors. Since accidents involving severe core damage are considered beyond the design basis this implies that DBAs in general have a collective frequency greater than $10^{-4}$ per year. It is noted CDF is a risk metric that has been defined in PRA standards ${ }^{[31]}$ and regulatory guides $^{[37]}$ in terms that are only meaningful for LWRs. Hence, CDF as a risk metric is not applicable to advanced non-LWRs. However, it provides a measure of the design objective for the frequency of beyond design basis accidents*

In the NRC Safety Goal Policy, the following performance guideline on the frequency of a large release is provided:

"Consistent with the traditional defense-in-depth approach and the accident mitigation philosophy requiring reliable performance of containment systems, the overall mean frequency of a large release of radioactive materials to the environment from a reactor accident should be less than 1 in 1,000,000 per year of reactor operation."

Although this large release frequency (LRF) metric is framed in terms of reactors with LWR containment systems, it is not unreasonable to conclude that the frequency of a large release can be used as a reactor technology metric, so long as the performance objective for a large release is defined. A proposed risk metric for demonstrating compliance with the Commission's LRF goal is included as part of the LMP LBE evaluation criteria as discussed in Section 4.

The NRC has not established a lower bound for the frequency of severe accidents that need to be considered. However, in general, the NRC does not require consideration of accidents that are not deemed to be "credible." Additionally, Regulatory Guide 1.174, Section A.2.4, states that an increase in core damage frequency of less than $10^{-6}$ per year and an increase in large early release frequency of $<10^{-7}$ per year are considered "very small" and consistent with the Commission's Safety Goal Policy. These criteria are repeated in Section III.2.2.5 of SRP 19, "Use of Probabilistic Risk Assessment in PlantSpecific Risk-Informed Decision-making: General Guidance." Additionally, SRP 19 states that a PRA may have a truncation limit that, depending on the level of PRA detail (module level, component level, or piece-part level), may be from $10^{-12}$ to $10^{-8}$ per reactor-year. Similarly, Regulatory Guide 1.200 , “An Approach for Determining the Technical Adequacy of Probabilistic Risk Assessment Results for Riskinformed Activities," Section 1.2.5, states that an external event may be screened out of a PRA if it can be

\footnotetext{
* Although some degree of core damage is assumed in the formulation of design basis source terms for evaluating containment leak rate and siting criteria, severe core damage phenomena such as direct containment heating, hydrogen conflagrations, steam explosions, over-pressurization, containment bypass, and other phenomena known to contribute to containment failure probability are explicitly considered as part of the design basis.
} 
shown that the mean value of the frequency of the corresponding design-basis hazard used in the plant design is less than $10^{-5}$ per year and that the conditional core-damage probability is less than $10^{-1}$, given the occurrence of the design-basis hazard. These guidelines indicate that events that have a frequency lower than $\sim 10^{-6}$ or $10^{-7}$ per year do not need to be evaluated, and that events with a frequency of less than about $10^{-8}$ may be screened from the PRA.

\section{A.1.4.5 UNITED KINGDOM SAFETY ASSESSMENT PRINCIPLES NUMERICAL TARGETS}

As previously discussed, the U.K. SAPs appear to roughly correspond to a collection of U.S. regulatory requirements, General Design Criteria, regulatory guides, standard review plan, and safety goals. The SAPs call for a fault analysis to be performed first for each separate nuclear facility that is submitted for a Generic Design Assessment and then for the site as a whole. The fault analysis supports three types of analyses that are expected to be done in an integrated fashion and in a manner that complements each other. The analyses are referred to as Design Basis Accident Analysis, PSA, ${ }^{*}$ and Severe Accident Analyses. The analyses are performed against a set of deterministic requirements set forth in the SAPs as well as numerical targets on the frequencies, consequences, and risks to individuals on-site and off-site. The numerical risk targets reflected in the SAPs are listed in Table A-6.

\footnotetext{
* IAEA and SAP documents use the term Probabilistic Safety Analysis (PSA) to mean the same thing as Probabilistic Risk
} Analysis according to the IAEA safety term glossary in Reference [38]. 
Table A-6. United Kingdom Safety Assessment Principles Numerical Risk Targets ${ }^{[39]}$

\begin{tabular}{|c|c|c|c|c|c|c|}
\hline No. & $\begin{array}{c}\text { Applicable State } \\
\text { or Event }\end{array}$ & Applicable to & $\begin{array}{l}\text { Facility or } \\
\text { Site Based }\end{array}$ & $\begin{array}{c}\text { Basic Safety } \\
\text { Objective (BSO) }\end{array}$ & $\begin{array}{c}\text { Basic Safety Limit } \\
\text { (BSL) }\end{array}$ & $\begin{array}{c}\text { Applicable Event } \\
\text { Frequency or } \\
\text { Consequence }\end{array}$ \\
\hline \multirow{2}{*}{1} & \multirow{2}{*}{$\begin{array}{l}\text { Normal } \\
\text { Operation }\end{array}$} & Any person on site (Radiation worker) & Site & $<1 \mathrm{mSv} /$ year & $<20 \mathrm{mSv} /$ year & \multirow{4}{*}{ Annual limits } \\
\hline & & Any person on site (Other employees) & Site & $<0.1 \mathrm{mSv} /$ year & $<2 \mathrm{mSv} /$ year & \\
\hline 2 & $\begin{array}{l}\text { Normal } \\
\text { Operation }\end{array}$ & Any group on site (Radiation workers) & Site & $<0.5 \mathrm{mSv} /$ year & $<10 \mathrm{mSv} /$ year & \\
\hline 3 & $\begin{array}{l}\text { Normal } \\
\text { Operation }\end{array}$ & Any person off site & Site & $0.02 \mathrm{mSv} /$ year & $1 \mathrm{mSv} /$ year & \\
\hline \multirow{6}{*}{4} & \multirow{6}{*}{$\begin{array}{l}\text { Design Basis } \\
\text { Accidents }\end{array}$} & \multirow{3}{*}{ Any person on site } & \multirow{6}{*}{ Facility } & \multirow{3}{*}{$<0.1 \mathrm{mSv} /$ event } & $20 \mathrm{mSv} /$ event & $>10^{-3} /$ year \\
\hline & & & & & 200mSv/event & $10^{-3}$ to $10^{-4} /$ year \\
\hline & & & & & 500mSv/event & $10^{-4}$ to $10^{-5} /$ year \\
\hline & & \multirow{3}{*}{ Any person off site } & & \multirow{3}{*}{$<0.01 \mathrm{mSv} /$ event } & $1 \mathrm{mSv} /$ event & $>10^{-3} /$ year \\
\hline & & & & & $10 \mathrm{mSv} / \mathrm{event}$ & $10^{-3}$ to $10^{-4} /$ year \\
\hline & & & & & 100mSv/event & $10^{-4}$ to $10^{-5} /$ year \\
\hline 5 & All accidents & Any person on site & Site & $<10^{-6} /$ year & $<10^{-4} /$ year & fatality \\
\hline \multirow{4}{*}{6} & \multirow{4}{*}{ All accidents } & \multirow{4}{*}{ Any Person on site } & \multirow{4}{*}{ Facility } & $<10^{-3} /$ year & $<10^{-1} /$ year & $2-20 \mathrm{mSv}$ \\
\hline & & & & $<10^{-4} /$ year & $<10^{-2} /$ year & $20-200 m S v$ \\
\hline & & & & $<10^{-5} /$ year & $<10^{-3} /$ year & $200-2000 \mathrm{mSv}$ \\
\hline & & & & $<10^{-6} /$ year & $<10^{-4} /$ year & $>2,000 \mathrm{mSv}$ \\
\hline 7 & All accidents & Any person off-site & Site & $<10^{-6} /$ year & $<10^{-4} /$ year & fatality \\
\hline \multirow{5}{*}{8} & \multirow{5}{*}{ All accidents } & \multirow{5}{*}{ Any Person off site } & \multirow{5}{*}{ Facility } & $<10^{-2} /$ year & $<1 /$ year & $0.1-1 \mathrm{mSv}$ \\
\hline & & & & $<10^{-3} /$ year & $<10^{-1} /$ year & $1-10 \mathrm{mSv}$ \\
\hline & & & & $<10^{-4} /$ year & $<10^{-2} /$ year & $10-100 \mathrm{mSv}$ \\
\hline & & & & $<10^{-5} /$ year & $<10^{-3} /$ year & $100-1,000 \mathrm{mSv}$ \\
\hline & & & & $<10^{-6} /$ year & $<10^{-4} /$ year & $>1,000 \mathrm{mSv}$ \\
\hline 9 & All accidents & All persons on and off-site & Site & $<10^{-7} /$ year & $<10^{-5} /$ year & $\begin{array}{l}\geq 100 \text { early or } \\
\text { latent fatalities }\end{array}$ \\
\hline
\end{tabular}


There is a long history in the development of numerical risk targets for evaluating nuclear reactor safety which started with a paper by Reginald Farmer. ${ }^{[64]}$ Dr. Farmer was actually responsible for performing the first PSA of reactor accidents in the 1960s which predated by a decade the first LWR PRA in WASH1400. What is significant to appreciate about the UK experience with the development of the SAPs is that they are reactor-technology neutral, having been applied to two different types of graphite moderated gascooled reactors, an operating LWR at Sizewell, and current being used to evaluate a number of ALWR designs as well as several liquid metal cooled fast reactor designs.

The numerical risk targets reflected in the SAPs provide extremely useful guidance in the development of the proposed F-C Target approach as explained more fully in Section 4 of this report.

\section{A.1.5 REGULATORY FOUNDATION PRECEDENT REVIEW SUMMARY}

The following observations and conclusions are made in this review of the regulatory foundation for selection of LBEs for advanced non-LWRs. These observations and conclusions shape the development of an approach for LBE selection that is provided in Section 4 of this report.

- Existing NRC Policy and Strategy statements fully support the greater use of RIPB practices. This vision is clearly articulated in NUREG-2150. There has been partial development of RIPB methods for the backfit, operation, oversight, and modification of existing LWRs. However, little or no guidance for RIPB decision making has been established for new, non-LWR advanced designs.

- The current U.S. regulations and regulatory guidance ("framework") for LWR-based designs do not include or provide a reproducible approach for selecting LBEs for advanced non-LWRs nor for ensuring that advanced non-LWRs of differing designs would be treated in a consistent manner for establishing their design and licensing bases.

- The only reactor technology-inclusive set of regulatory documents that was identified in this regulatory review is that reflected in the U.K. SAPs. The SAPs include numerical targets for evaluating LBE frequencies and consequences which differentiate between those to be applied to each reactor unit and those which apply to the site as a whole. Different targets are expressed for regulatory evaluation boundaries and design objectives, thereby capturing the notion that risk are not to be used a strict pass-fail acceptance test.

- PBMR and NGNP projects, as well as the approach used for PRISM for LBE selection, provide an appropriate baseline from which to develop the LBE selection process for advanced reactor design and licensing. An LBE selection approach proposed in NUREG-1860 was also reviewed for insights to help define desirable attributes of an effective LBE selection process. This regulatory foundation review provides guidance for refining and advancing these approaches.

- The RIPB approach advanced in the MHTGR, PBMR and NGNP projects has been reflected in a design standard for MHRs in ANS 53.1. This standard provides specific design criteria for implementing the approach that is consistent with the approach described in the NGNP white papers. These include criteria for evaluating the adequacy of defense-in-depth which contributes to the deterministic input to RIPB design decisions.

- There are a number of international precedents, including those from the U.S., IAEA, and the U.K. SAPs, and reflected in the NRC reviews of MHTGR, PRISM, and NGNP, that support the view that LBE selection is best accomplished through a risk-informed and performance-based process which includes both deterministic and probabilistic inputs and preserves the principle of defense-in-depth.

- A key challenge of any LBE selection process is to systematically define the initiating events that are appropriate for the reactor design, and the event sequences that realistically model the plant 
response to the initiating events. This is necessary in order to derive the appropriate and limiting DBAs for that design. Simply removing inapplicable events from existing LWR events is not sufficient to define the events that are uniquely appropriate for a given design.

- The LBE definition and selection process must be clear in making the distinction between initiating events and event sequences. A given initiating event may result in different event sequences each having a different frequency of occurrence and level of severity in challenging the reactor safety defenses. Simply assuming the "worst active single failure" and concurrent loss of offsite power in combination with an initiating event does not necessarily yield the appropriate limiting accidents to define the licensing basis.

- As emphasized in NUREG-1860, PRA plays an important role in the identification and evaluation of uncertainties in the definition of event sequences and in the estimation of their frequencies and consequences. This information on sources of uncertainty and their influences on the risk assessment are important inputs to establishing adequate consideration of the principles of defense-in-depth in the selection and evaluation of LBEs and other RIPB decisions.

- In order to provide the technical basis for managing the risks of accidents that involve two or more reactors or radionuclide sources, by preventing and mitigating such accidents, it is necessary to consider such accidents in the definition of LBEs and to measure frequencies on a per (multi-reactor module) plant ${ }^{*}$-year basis, rather than reactor-year basis.

- The development of F-C Targets for the LMP project greatly benefits from the approach most recently advanced in the NGNP LBE white paper as well as similar frequency-consequence criteria originally proposed by Farmer. Useful guidance is also available from NUREG-1860 the U.K. SAPs for event consequences, frequencies and threshold for event evaluation.

- A key challenge in interpreting the current U.S. regulations for limiting radiological exposures for normal operation and LBEs is the lack of explicit numerical criteria for categorizing events by expected frequency of occurrence. However, the classification of LBEs into AOOs, DBEs, and BDBEs based on expected frequency of occurrence is consistent with LBE classifications that were identified in this regulatory review including NGNP, PRISM, NUREG-1860, NUREG2150, Yucca Mountain Pre-Closure Safety Analysis, and the U.K. SAPs.

- There are a number of NRC criteria that explicitly constrain the risk and/or allowable consequences of radiological releases from nuclear power plants. These criteria include requirements to evaluate the adequacy of the proposed design of the plant against specific limits. Some of the regulatory dose requirements are intended for evaluation of individual events, whereas others are expressed in terms of annual exposure limits, frequency of a given magnitude of release, and individual risks for the population in the vicinity of the plant site. The review of these criteria that was performed in the NGNP LBE white paper ${ }^{[1]}$ has been extended in this report and has yielded some new insights that are reflected in the proposed LBE selection approach as discussed in the next section.

The above key points have been used to guide the development of the LBE selection process as discussed more fully in Section 4.

\footnotetext{
* "Plant," as the term is used in this document means a nuclear plant that may or may not employ a modular design. "Modular design" means a nuclear power station that consists of two or more essentially identical nuclear reactors (modules) and each module is a separate nuclear reactor capable of being operated independent of the state of completion or operating condition of any other module co-located on the same site, even though the nuclear power station may have some shared or common systems. ${ }^{[2]}$
} 


\section{A.2 REFERENCES}

[1] Idaho National Laboratory, "Next Generation Nuclear Plant Licensing Basis Event Selection White Paper," INL/EXT-10-19521, September 2010, [ADAMS Accession No. ML102630246].

[2] 10 CFR 52.1, "Licenses, Certifications, and Approvals for Nuclear Power Plants - Definitions," 2015.

[3] NUREG-0800, Standard Review Plan, Chapter 15.0, "Introduction - Transient and Accident Analyses," Revision 3 March 2007.

[4] 10 CFR 50, Appendix A, "General Design Criteria for Nuclear Power Plants," Code of Federal Regulations, Office of the Federal Register, 2007.

[5] 10 CFR 50.62, "Requirements for reduction of risk from anticipated transients without scram (ATWS) events for light-water-cooled nuclear power plants," 2015.

[6] 10 CFR 50.63, "Loss of all alternating current power," 2015.

[7] 10 CFR 50.44, "Combustible gas control for nuclear power reactors,", 2015.

[8] 10 CFR 50.150, "Aircraft impact assessment," 2015.

[9] U.S. Nuclear Regulatory Commission, "Policy Statement on the Regulation of Advanced Reactors," 73 FR 60612, October 14, 2008.

[10] U.S. Nuclear Regulatory Commission, "Policy Statement on Use of Probabilistic Risk Assessment Methods in Nuclear Regulatory Activities" 60 FR 42622, August 16, 1995.

[11] U.S. Nuclear Regulatory Commission, 'Policy Statement on Safety Goals for Operation of Nuclear Power Plants," 51 FR 28044 August 21, 1986.

[12] U.S. Nuclear Regulatory Commission, "Policy Statement on Severe Reactor Accidents Regarding Future Designs and Existing Plants," 50 FR 32138, August 8, 1985.

[13] SECY 2002-0076, "Semi-Annual Update of the Future Licensing and Inspection Readiness Assessment (FLIRA)," May 8, 2002.

[14] SECY 2003-0047, "Policy Issues Related to Licensing Non-Light Water Reactor Designs," U.S. Nuclear Regulatory Commission, March 28, 2003.SECY 2002-0139.

[15] SRM 2003-0047, "Staff Requirements Memorandum for SECY 03-0047-Policy Issues Related to Licensing Non-Light Water Reactor Designs," U.S. Nuclear Regulatory Commission, June 26, 2003.

[16] SECY-2005-0006, "Second Status Paper on the Staff's Proposed Regulatory Structure for New Plant Licensing and Update on Policy Issues Related to New Plant Licensing,” January 7, 2005.

[17] SECY-2010-0034, "Potential Policy, Licensing, and Key Technical Issues for Small Modular Nuclear Reactor Designs," March 28, 2010.

[18] SECY-2011-0079, "License Structure for Multi-Module Facilities Related to Small Modular Nuclear Power Reactors," June 12, 2011.

[19] NUREG-0800, Standard Review Plan, Chapter 19.0, "Probabilistic Risk Assessment and Severe Accident Evaluation for New Reactors," Revision 3, December 2015.

[20] SECY 2016-0012, "Accident Source Terms and Siting for Small Modular Reactors and Non-Light Water Reactors," February 7, 2016.

[21] NUREG-1860, "Feasibility Study for a Risk-Informed and Performance-Based Regulatory Structure for Future Plant Licensing," U.S. Nuclear Regulatory Commission, December 2007. 
[22] NUREG-2150, “A Proposed Risk Management Regulatory Framework,” U.S. Nuclear Regulatory Commission, April 2012.

[23] U.S. Nuclear Regulatory Commission, "The Near-Term Task Force Review of Insights from the Fukushima Dai-Ichi Accident," July 12, 2011.

[24] NUREG/BR-0303, Guidance for Performance Based Regulation, U.S. Nuclear Regulatory Commission, December 2002.

[25] ANS-53.1-2001, "Nuclear Safety Design Process for Modular Helium-Cooled Reactor Plants," American Nuclear Society, December 2011.

[26] Idaho National Laboratory, "Next Generation Nuclear Plant Defense-in-Depth Approach White Paper," INL/EXT-09-17139, March 2009, [ADAMS Accession No. ML102630246].

[27] Department of Energy, "Yucca Mountain Repository Safety Analysis Report," Chapters 1.6 - 1.9 and 1.14, Docket No. 63-001, DOE/RW-0573, Rev. 1, February 2009.

[28] NUREG-2108, “Technical Evaluation Report on the Content of the U.S. Department of Energy's Yucca Mountain Repository License Application: Pre-closure Volume: Repository Safety Before Permanent Closure," Nuclear Regulatory Commission, Office of Nuclear Material Safety and Safeguards, September 2011.

[29] 10 CFR 63, "Disposal of High-Level Radioactive Wastes in a Geologic Repository at Yucca Mountain, Nevada," 2016.

[30] NUREG-1804, "Yucca Mountain Review Plan," Revision 2, July 2003.

[31] ASME/ANS RA-Sb-2013, “Addenda to ASME/ANS RA-S-2008 Standard for Level 1/Large Early Release Frequency Probabilistic Risk Assessment for Nuclear Power Plant Applications," American Society of Mechanical Engineers and American Nuclear Society, September 2013.

[32] ASME/ANS RA S-1.4-2013, "Probabilistic Risk Assessment Standard for Advanced Non-LWR Nuclear Power Plants," American Society of Mechanical Engineers and American Nuclear Society, December 2013.

[33] 10 CFR 63.111, "Performance objectives for the geologic repository operations area through permanent closure," 2015.

[34] 10 CFR 63.112, "Requirements for Pre-closure Safety Analysis of the Geologic Repository Operations Area," 2015.

[35] SRM 90-16, "Staff Requirements Memorandum for SECY 90-16 Evolutionary Light Water Reactor Certification Issues and Their Relationship to Current Regulatory Requirements," January 1990.

[36] Regulatory Guide 1.174, "An Approach for Using Probabilistic Risk Assessment in Risk-informed Decisions on Plant Specific Changes to the Current Licensing Basis," Federal Register, U.S. Nuclear Regulatory Commission, Revision 2, May 2011.

[37] Regulatory Guide 1.200, "An Approach for Determining the Technical Adequacy of Probabilistic Risk Assessment Results for Risk-informed Activities,” Federal Register, U.S. Nuclear Regulatory Commission, Revision 2, March 2009.

[38] International Atomic Energy Agency, "Safety Standards Series: Safety of Nuclear Power Plants Design Requirements,” NSR-1, September 2000.

[39] United Kingdom Office of Nuclear Regulation, "Safety Assessment Principles for Nuclear Facilities," 2014 Edition Revision 0.

[40] U.S. Department of Energy, Preliminary Safety Information Document for the Standard MHTGR, DOE-HTGR-86-024, September 1988. 
[41] U.S. Department of Energy, "Probabilistic Risk Assessment for the Standard Modular High Temperature Gas-Cooled Reactor,” DOE-HTGR-86-011, Revision 5, April 1988.

[42] NUREG-1338, "Draft Preapplication Safety Evaluation Report for the Modular High Temperature Gas-Cooled Reactor,” U.S. Nuclear Regulatory Commission, March 1989.

[43] NUREG-1368, "Preapplication Safety Evaluation Report for the Power Reactor Innovative Small Module (PRISM) Liquid-Metal Reactor, Final Report,” U.S. Nuclear Regulatory Commission, February 1994.

[44] Edward G. Wallace letter to NRC Document Control Desk, "PBMR White Paper: LBE Selection," July 3, 2006.

[45] NRC letter to PBMR, "Requests for Additional Information Regarding Pebble Bed Modular Reactor (PBMR) Pre-Application White Papers," September 24, 2007.

[46] PBMR Limited letter to NRC, "Response to Requests for Additional Information," March 21, 2008.

[47] Exelon Generation Company Letter, Subject: "Proposed Licensing Approach for the Pebble Bed Modular Reactor in the United States," January 31, 2002.

[48] U.S. Nuclear Regulatory Commission Letter, Subject: "NRC Staff's Preliminary Findings Regarding Exelon Generation's (Exelon's) Proposed Licensing Approach for The Pebble Bed Modular Reactor (PBMR)," March 26, 2002. See also SECY-95-0299, "Licensing Approach for Exelon's Pebble Bed Modular Reactor,” December 19, 1995.

[49] INL/EXT-13-28205, “NRC Licensing Status Summary Report for NGNP," Idaho National Laboratory, November 2014.

[50] Letter from J. Sam Armijo, Chairman U.S. Nuclear Regulatory Commission Advisory Committee on Reactor Safeguards to Mr. R.W. Borchardt, Executive Director for Operations, U.S. Nuclear Regulatory Commission Letter, "Next Generation Nuclear Plant (NGNP) Key Licensing Issues", May 15, 2013.

[51] SECY 2011-0152, "Development of an Emergency Planning and Preparedness Framework for Small Modular Reactors," October 28, 2011.

[52] 10 CFR 20, "Standards for Protection against Radiation Subpart C, Occupational Dose Limits", 2016.

[53] 10 CFR 20, "Standards for Protection against Radiation Subpart D, Radiation Dose Limits for Individual Members of the Public," 2016.

[54] 10 CFR 50, Appendix I, "Numerical Guides for Design Objectives and Limiting Conditions for Operation to Meet the Criterion 'As Low as is Reasonably Achievable' for Radioactive Material in Light-Water-Cooled Nuclear Power Reactor Effluents,” 2015.

[55] 10 CFR 52, Licenses, Certifications, And Approvals for Nuclear Power Plants, Subpart C "Combined Licenses," 2016.

[56] 10 CFR 52.79, "Contents of applications; technical information in final safety analysis report," 2015.

[57] 40 CFR 190, "Environmental Radiation Protection Standards for Nuclear Power Operations," 2016.

[58] 10 CFR 100, "Reactor Site Criteria (Subpart B, Evaluation Factors for Stationary Power Reactor Site Applications on or After January 10, 1997)," 1996.

[59] 10 CFR 50.34, "Contents of Applications: Technical Information - Radiological Dose Consequences," 2015. 
[60] 10 CFR 50.34a, "Design objectives for equipment to control releases of radioactive material in effluents - nuclear power reactors," 2015.

[61] 40 CFR 190.10, "Standards for normal operations," 2016.

[62] Idaho National Laboratory, "Next Generation Nuclear Plant Probabilistic Risk Assessment White Paper,” INL/EXT-11-21270, Idaho National Laboratory, September 2011.

[63] NUREG-0800, Standard Review Plan, Chapter 15.0.3 "Design Basis Accident Radiological Consequence Analyses for Advanced Light Water Reactors," March 2007.

[64] Farmer, F., "Reactor Safety and Siting: A Proposed Risk Criterion," Nuclear Safety; pp. 539-48, 1967.

[65] American Nuclear Society, ANSI/ANS-53.1-2011, "Nuclear Safety Design Process for Modular Helium-Cooled Reactor Plants," December 21, 2011.

[66] NEDO-33201, Revision 6, ESBWR Design Certification Probabilistic Risk Assessment, Chapter 10.4 Comparison of Results to Goals, (Adams Accession No. ML102880536).

[67] U.S. Department of Energy, Licensing Basis Events for the Standard MHTGR, DOE-HTGR-86034, February 1987.

[68] GE Hitachi Nuclear Energy, "Final Scientific/Technical Report: Development/Modernization of an Advanced Non-LWR Probabilistic Risk Assessment,” Federal Grant DE-NE0008325, 2017.

[69] Letter from Mario V. Bonaca, Chairman U.S. Nuclear Regulatory Commission Advisory Committee on Reactor Safeguards to Honorable Nils Diaz, Chairman, U.S. Nuclear Regulatory Commission Letter, "Options and Recommendations for Policy Issues Related to Licensing NonLight Water Reactor Designs,” April 22, 2004.

[70] SECY 2013-0029, "History of The Use and Consideration of the Large Release Frequency Metric by The U.S. Nuclear Regulatory Commission", March 22, 2013.

[71] NUREG-2122, Glossary of Risk-Related Terms in Support of Risk-Informed Decisionmaking, November 2013.

[72] NRC Glossary, from https://www.nrc.gov/reading-rm/basic-ref/glossary.html. 


\section{APPENDIX B-TECHNICAL BASIS OF THE LMP FREQUENCY-CONSEQUENCE TARGET}

\section{B.1 LMP Documentation}

\section{Overview}

The purpose of the LMP Frequency-Consequence (F-C) Target, shown in Figure B-1, is to evaluate the risk significance of individual Licensing Basis Events (LBEs). The risk of an event includes the frequency of occurrence and its consequences and is often expressed as the product of the frequency and consequence. The F-C Target represents a design objective to control the risks of a range of off normal and unplanned events including those that may occur in the lifetime of the plant (Anticipated Operational Occurrences or AOOs), infrequent events considered in formulating the plant design basis (Design Basis Events or DBEs), and rare events beyond the design basis (Beyond Design Basis Events or BDBEs).

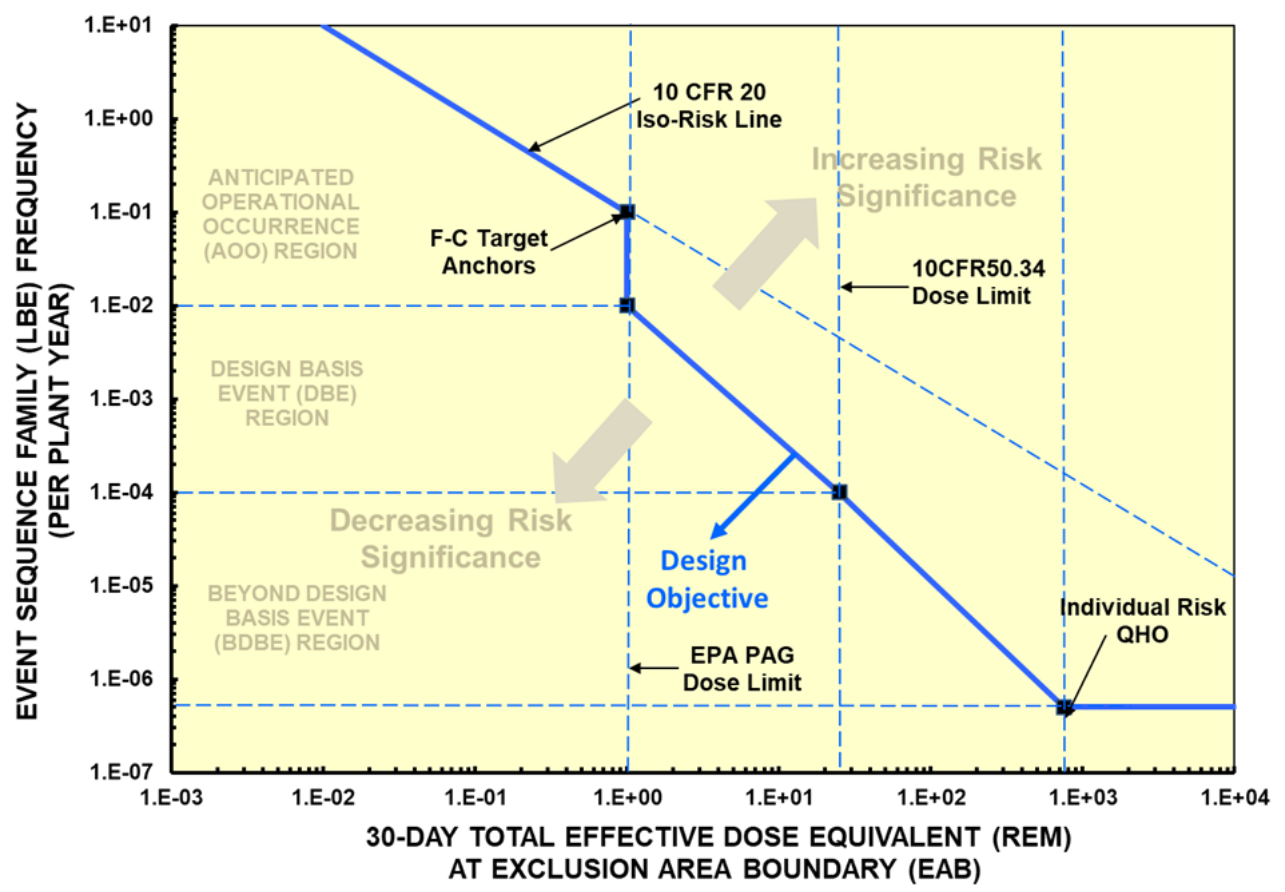

Figure B-1. LMP F-C Target for Evaluating the Risk Significance of Licensing Basis Events

The philosophy behind the definition of the F-C Target is reflected in the following statement in the SRP Chapter 15 that is used to develop acceptance criteria for AOOs and postulated accidents.

"If the risk of an event is defined as the product of the event's frequency of occurrence and its consequences, then the design of the plant should be such that all the AOOs and postulated accidents produce about the same level of risk (i.e., the risk is approximately constant across the spectrum of AOOs and postulated accidents). This is reflected in the general design criteria (GDC), which generally prohibit relatively frequent events (AOOs) from resulting in 
serious consequences, but allow the relatively rare events (postulated accidents) to produce more severe consequences."

In formulating the LMP F-C Target, a number of key inputs were considered including:

- $\quad 10$ CFR 20 which limits public exposures from normal operation and anticipated events to $100 \mathrm{mrem} /$ year

- $\quad$ The 1-rem EPA Protective Action Guide dose, which if exceeded would lead to offsite protective actions

- $\quad 10$ CFR 50.34 which limits exposures from the most severe and least likely design basis accidents to 25 rem

- $\quad$ NRC Safety goal Quantitative Health Objectives which limit the individual risks to the population within 1 mile of the site boundary to $5 \times 10^{-7} /$ year

The F-C target is comprised of an iso-risk curve in the AOO region covering anticipated events with a frequency of $10^{-2} /$ plant year and above limiting the combination of frequency and dose to $100 \mathrm{mrem} /$ year. The F-C Target caps the dose levels at the EPA PAG level so as to avoid the need for offsite protective actions for AOO events. In the DBE and BDBE regions, the F-C Target is set so that the acceptable risk target decreases with decreasing frequency to incorporate the principle of risk aversion that was identified in the first example of a F-C Target by Farmer. ${ }^{1}$ This is reflected in Table A-1. As seen in this table, the risk target at the lower end of the DBE region at $10^{-4}$ /plant-year is set to 40 times lower than the AOO risk target and the risk target at the lower end of the BDBE region is more than 260 times lower than the AOO risk target. Hence the risk management philosophy reflected in the LMP F-C Target is conservative in relation to the quoted SRP statement. It is important to keep in mind that the points along the F-C Target do not represent acceptable dose limits, but rather acceptable risk limits. The dose levels on along the target should not be evaluated outside the context of the event frequency.

Table A-1. Risk Target to Risk Limit Ratio for LBE Categories

\begin{tabular}{|c|c|c|c|c|}
\hline LBE Category & $\begin{array}{c}\text { Frequency } \\
\text { (per plant-year) }\end{array}$ & $\begin{array}{c}30 \text { Day Site } \\
\text { Boundary Dose } \\
\text { Limit (rem) }\end{array}$ & $\begin{array}{c}\text { Risk Limit } \\
\text { (mrem/plant- } \\
\text { year) }\end{array}$ & $\begin{array}{c}\text { Ratio AOO Risk } \\
\text { Target to Risk } \\
\text { Limit }\end{array}$ \\
\hline AOO & 1 & 0.1 & 100 & 1 \\
\hline DBE & $1.00 E-04$ & 25 & 2.5 & 40 \\
\hline BDBE & $5.00 E-07$ & 750 & 0.375 & 267 \\
\hline
\end{tabular}

More details on the derivation of the LMP F-C Target and comparisons with alternative targets are found in the Section 3 of this report.

In the following, some frequently asked questions regarding the bases for the LMP risk targets are addressed.

\footnotetext{
${ }^{1}$ Farmer, R., "Reactor Safety and Siting: A Proposed Risk Criterion," Nuclear Safety 1967; pp. 539-48.
} 


\section{B.2 Basis for Lower Limit of BDBE Region at 5×10-7/plant-year}

The technical basis for excluding events of lower frequency in the BDBE region is based on the LMP finding that events of lower frequency, regardless of consequence level, are too rare to challenge the capability to meet the NRC Safety Goal Quantitative Health Objectives for individual risk. Although events whose upper bound frequencies do not exceed $5 \times 10^{-7} /$ plant year are not classified as BDBEs, such events are not excluded from consideration in the LMP methodology. As stated in NEI-18-04:

"Event sequences with frequencies less than $5 \times 10^{-7}$ /plant-year are retained in the PRA results and used to confirm there are no cliff edge effects. They may also be taken into account in the RIPB evaluation of defense-in-depth.

As stated, events below this cut-off will not significantly contribute to the QHO individual risk metric and this conclusion is independent of the level of consequences associated with the event. This conclusion is supported by the industry's previous conversations with the staff and by the example that is discussed in response to the question about the F-C Target anchor point at the bottom of the BDBE region that is discussed below,

Although the AOOs, DBEs, and BDBEs are classified based on the mean frequency estimates of the underlying event sequences, the uncertainties about these mean frequency estimates are considered to ensure that the classification is robust in light of uncertainties. This is reflected in the following statements made in NEI 18-04 in the text for Task 7a for Figure 3-1:

The mean values of the frequencies are used to classify the LBEs into AOOs, $D B E s$, and BDBE categories. However, when the uncertainty bands ${ }^{2}$ defined by the $5^{\text {th }}$ percentile and $95^{\text {th }}$ percentile of the frequency estimates straddles a frequency boundary, the $L B E$ is evaluated in both LBE categories. An LBE with mean frequency above $10^{-2} /$ plant-year and $5^{\text {th }}$ percentile less than $10^{-2} /$ plant-year is evaluated as an AOO and DBE. An LBE with a mean frequency less than $10^{-4} /$ plant-year with a $95^{\text {th }}$ percentile above $10^{-4} /$ plant-year is evaluated as a BDBE and a DBE. An event sequence family with a mean frequency less than $5 \times 10^{-7} /$ plant year but with a $95 \%$ tile frequency estimate above $5 \times 10^{-7} /$ plant-year is evaluated as a BDBE. Uncertainties about the mean values are used to help evaluate the results against the frequencyconsequence criteria and to identify the margins against the criteria. The uncertainties about these means are considered as part of the RIPB DID evaluation in Task 7 e.

\footnotetext{
${ }^{2}$ It is recognized that the PRA may not fully resolve the impacts of all sources of uncertainty, such as modeling uncertainty. The LMP approach to PRA recommends following the guidance in NUREG-1855 to address uncertainties. Uncertainties not quantified in the PRA are important inputs to the evaluation of defense-in-depth adequacy in Task 7e.
} 
It should be noted that this argument has been previously reviewed by NRC staff and the ACRS during the NGNP reviews and more recently as part of the NRC staff's review of the LMP methodology.

The role of the use of the frequency bins within the LMP methodology is put into the following context with the following statements in DG-1353:

"The F-C target and related discussions in NEI 18-04 include a frequency of $5 \times 10^{-7}$ per plant-year to define the lower range of beyond design basis events. This demarcation of lowest event frequencies on the F-C target and category definitions should not be considered a hard and fast cutoff but should instead be considered in the context of other parts of the methodology described in NEI 18-04. These other considerations include the role of the integrated decision-making panel, DID assessments, accounting for uncertainties, and assessing for potential cliff-edge effects."

When interpreting the frequency bins for the LBE categories in NEI 18-04 the following considerations should be taken into account:

- $\quad \mathrm{AOO}, \mathrm{DBE}$, and BDBE frequencies are measured on a per plant-year basis to accommodate the inclusion of event sequences that may involve two or more reactor modules or radionuclide sources. For event sequences that impact individual reactor modules, a frequency of $5 \times 10^{-7} /$ plant-year would be equivalent to $5 \times 10^{-8} /$ reactor-year for a 10 module plant.

- $\quad$ Any $\mathrm{AOO}, \mathrm{DBE}$, or $\mathrm{BDBE}$ is considered risk significant if its frequency at a given level of consequence is within $1 \%$ of the F-C Target (see Figure 3-4 in NEI 18-04). Risk significant LBEs are given special emphasis in the evaluation of defense-in-depth (DID) adequacy.

- Uncertainty ranges surrounding the estimation of LBE frequencies and consequences that are found to be risk significant, including modeling uncertainties, are addressed in the evaluation of DID adequacy. The identification of meaningful DID protective strategies to compensate for risk-significant uncertainties is an integral part of the LMP methodology.

In summary, the F-C Target is a design objective to control the risks of individual LBEs. Cumulative risk targets within the LMP methodology are used to control the total integrated risks from all the LBEs. Risk targets are not regulatory limits. The regulatory limits for implementing the LMP methodology are derived from the NRC regulations and the Safety Related Design Criteria that are defined within the LMP methodology for Safety Related SSCs. In addition, all the safety significant SSCs which are covered in the SR and NSRST SSCs will have reliability and capability targets supported by a risk-informed and performance based evaluation of defense-in-depth adequacy to support each reactor's safety case. Hence, the use of the F-C Target is just one element of a risk-informed licensing process. 


\section{B.3 Basis for the F-C Target Anchor Point at a Frequency of $5 \times 10^{-7} /$ plant-year and Dose of 750 rem}

To put into context the role of the F-C Target in implementing the LMP methodology, the following statement is made in DG-1353.

"The staff emphasizes the cautions in NEI 18-04 that the F-C target figure does not depict acceptance criteria or actual regulatory limits. The anchor points used for the figure are surrogates for other measures that may be expressed in different units, time scales, or distances. The F-C target provides a reasonable approach to be used within a broader, integrated approach to determine risk significance and support SSC classification and confirm the adequacy of DID."

The frequency basis for setting the lower limit for BDBEs of $5 \times 10^{-7} /$ plant-year was explained in the answer to the previous question. The dose of 750 rem at this frequency was selected on the basis that it would yield a high probability of death due to prompt radiation syndrome to a person standing at the $\mathrm{EAB}$ for 30 days without any evacuation or sheltering, however the frequency was sufficiently low at this low level to challenge the NRC safety goals. This limit was selected as a reasonable and conservative dose level to ensure that an event at this frequency or lower and this dose or higher would not challenge the capability to meet the NRC Safety Goal QHO for individual risk. This QHO is applicable to all the individuals located within 1 mile of the site boundary, not just to individuals that may be right on the site boundary. That is, the dose target at the specified frequency level provides a point on the F-C Target as a quantitative risk measure that assures the capability to meet the QHO.

In order to estimate the individual risk for comparison to the QHOs it is necessary to estimate the frequency of each event sequence, the doses to each individual within 1 mile of the site boundary, and the number of early fatalities in this region due to these exposures. After all the fatalities are estimated, weighted by the frequency of each event sequence and summed, the early fatality risk is divided by the initial population at risk to obtain the individual risk for comparison to the QHO which is $5 \times 10^{-7}$ per person per year within 1 mile. This risk metric can expressed as the sum of the frequencies of the LBEs times the fraction of the population within 1 mile of the site boundary that incurs a prompt fatality. For a given LBE to just reach the individual risk QHO its frequency times the fraction of the 1 mile population that incurs prompt fatality must exceed $5 \times 10^{-7} /$ year. If the frequency is, say, $5 \times 10^{-5} /$ year the fraction of the population that incurs prompt fatality would have to be above 0.01 to exceed the QHO. If the frequency is $5 \times 10^{-7} /$ year, the fraction would have to be 1.0 just to reach the QHO.

If the dose at the site boundary at the center line of the plume is $750 \mathrm{rem}$, the doses will fall off rapidly down the center line of the plume from the site boundary to 1 mile beyond that. The dose will also fall with angle off the centerline of the plume and individuals outside the plume will get no dose. If it is assumed that the plume has a width of $24^{\circ}$, the fraction of the population inside the plume would be about $7 \%$, leaving the remaining $93 \%$ outside the plume with no dose. 
To evaluate the dose behavior inside the plume without considering the effects of any evacuation or sheltering, a rough estimate was made using information from NUREG-1140 using a conservative atmospheric dispersion model consistent with weather conditions that could yield high radiation doses. Figure B-2 provides a chart from NUREG-1140 showing that radiation doses, which are proportional to chi/Q, would be highest with Category F, $0.1 \mathrm{~m} / \mathrm{sec}$ wind speed and no buoyancy assumed.

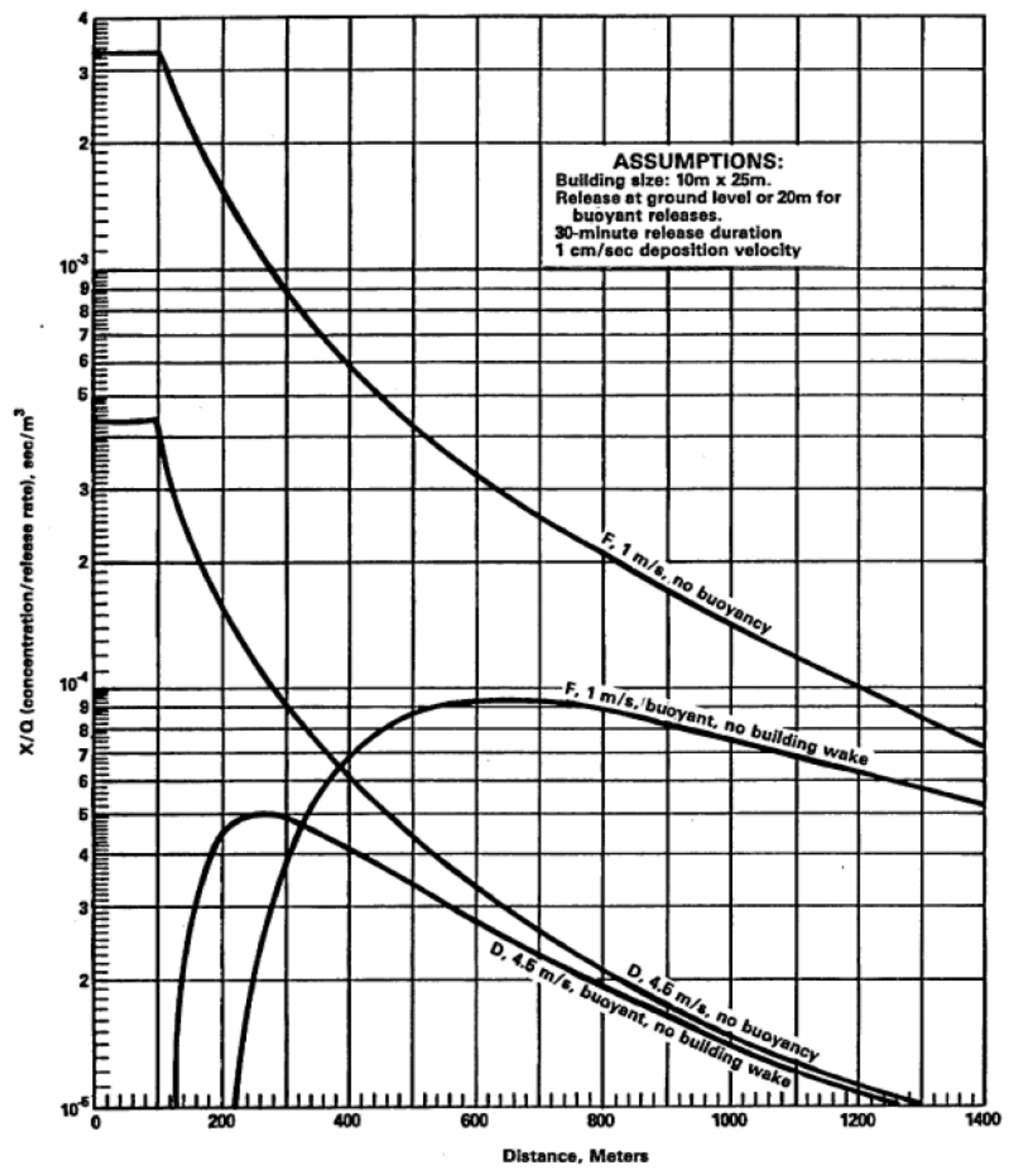

Figure B-2. Figure from NUREG-1140 - Atmospheric Dispersion (chi/Q) Versus Distance

Using this weather model, a plot of the dose vs. distance behavior down the center line of the plume was obtained by fixing the dose at an assumed 400 meter EAB to 750 rem and using the 
conservative chi/Q model described above. The result is shown in Figure B-3. As seen in this figure, the dose falls off very rapidly with distance over the 1 mile area for the QHO calculation. The dose drops below the threshold for early fatality of 300rem at about 800 meters. This means that less than $1 \%$ of the area within 1mile of the EAB would be subject to doses above 300rem. This is obtained by comparing the area from $400 \mathrm{~m}$ to $800 \mathrm{~m}$, to that of the entire QHO "doughnut" from $400 \mathrm{~m}$ to $2000 \mathrm{~m}$. At the midpoint of the 1 mile area which is about 1200 meters from the release the dose drops down to about $125 \mathrm{rem}$ and at the edge of the 1 mile region it is down to about 30rem. All these doses are down the center line of the plume.

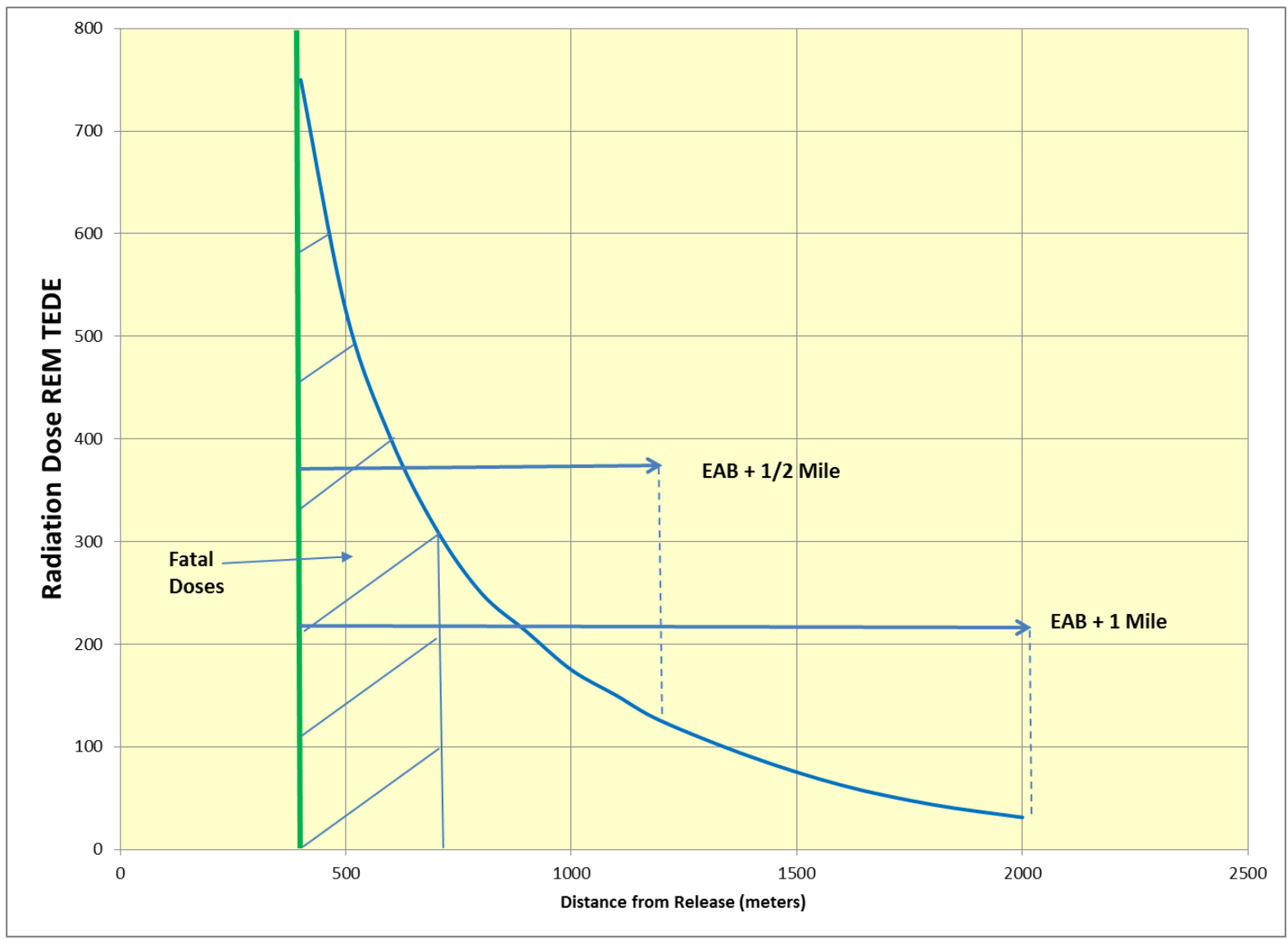

Figure B-3. Dose vs. Distance for 750rem at 400m EAB with Category F Stability, Low Wind Speed, and No Buoyancy

In the above analysis it is seen that if the dose at the plume centerline is at 750rem the vast majority of the population within 1 mile of the site boundary would not get a dose sufficient to produce early fatality. Less than $10 \%$ would get any dose and less than $1 \%$ would get a dose above 300rem.

It is noted that the doses are carried out for the F-C Target for a 30-day exposure, whereas doses for calculating the QHOs are typically limited to 24 hour exposures. Finally, at the frequency of $5 \times 10^{-7}$ per year where the 750rem dose is applied to draw the F-C Target, it would be possible to expose a large fraction of the 1 mile population to potentially fatal doses and still meet the 
QHOs. Hence, the selection of the risk target with the dose level of 750rem at the $5 \times 10^{-7} /$ year frequency level provides reasonable confidence that the resulting QHO for individual risk would be met. 


\section{APPENDIX C-LMP DOCUMENTATION AND FREQUENTLY ASKED QUESTIONS}

\section{C.1 LMP Documentation}

The LMP team prepared independent reports on each of the four major LMP elements. Additionally, the LMP team produced a narrative report describing the processes, events, and documents involved in producing the ultimate project deliverable product, NEI 18-04 "RiskInformed Performance-Based Technology Inclusive Guidance for Non-Light Water Reactor Licensing Basis Development." Finally, the LMP team produced a report based on the experiences of early adopters of the LMP RIPB process which includes best practices, lessons learned, and frequently asked questions and responses. Table C-1 lists the Southern Company document numbers of each of these reports. The documents are available via the DOE's Office of Scientific and Technical Information (OSTI) public document repository (https://www.osti.gov).

Table C-1. LMP Reports and Document Numbers

\begin{tabular}{|c|c|c|}
\hline Report Title & $\begin{array}{l}\text { Southern Company } \\
\text { Document Number }\end{array}$ & $\begin{array}{c}\text { DOE OSTI } \\
\text { Document Number }\end{array}$ \\
\hline Selection and Evaluation of Licensing Basis Events & SC-29980-100 Rev 1 & TBD \\
\hline Probabilistic Risk Assessment Approach & SC-29980-101 Rev 1 & TBD \\
\hline $\begin{array}{l}\text { Safety Classification and Performance Criteria for } \\
\text { Structures, Systems, and Components }\end{array}$ & SC-29980-102 Rev 1 & TBD \\
\hline $\begin{array}{l}\text { Risk-Informed and Performance-Based Evaluation of } \\
\text { Defense-in-Depth Adequacy }\end{array}$ & SC-29980-103 Rev 1 & TBD \\
\hline Final Project Report & SC-29980-105 Rev. 1 & TBD \\
\hline $\begin{array}{l}\text { LMP Lessons Learned, Best Practices, and Frequently } \\
\text { Asked Questions }\end{array}$ & SC-29980-106 Rev 0 & TBD \\
\hline
\end{tabular}

\section{Licensing Basis Event Selection Approach}

Inputs to the selection of LBEs are derived from a PRA of an advanced non-LWR plant. These inputs together with deterministic inputs, such as design selections and selection of SafetyRelated (SR) SSCs, are used as part of the selection and evaluation of LBEs. As part of the LBE selection and evaluation process described in the LBE report, the engineering and safety analysis effort will result in a selection of a set of SR SSCs that are necessary and sufficient to perform the PRA Safety Functions (PSFs) required to keep the Design Basis Events (DBEs) within the Frequency-Consequence (F-C) target, and to prevent any high-consequence Beyond Design Basis Event (BDBE) from migrating into the DBE region and exceeding the F-C Target. The SR SSCs are then relied upon to mitigate all the Design Basis Accidents (DBAs) within the dose limits of 10 CFR 50.34 using conservative assumptions. 


\section{Probabilistic Risk Assessment Approach}

This report outlines the approach to develop a PRA for advanced non-LWR plants in support of risk-informed and performance-based (RIPB) applications. Future advanced non-LWR license applications will include a design-specific PRA that is capable of supporting the applications for NRC permit(s) or license(s). When introduced at an early stage of the design, the PRA is expected to result in a more efficient risk management process. This report outlines the relevant regulatory policy and guidance for this type of PRA, describes the approach to be followed for the development of the PRA, and sets forth PRA topics that need to be addressed in order to facilitate successful design and more safety focused preparation and review of the license application.

\section{SSC Safety Classification and Performance Requirements Approach}

Information developed from and used in the development of the PRA to define event sequences and evaluate their frequencies and consequences is an input to the SSC safety classification and development of SSC performance targets. Information from the PRA is used to establish the necessary and sufficient conditions of SSC capability and reliability in order for LBE frequencies, consequences, and uncertainties to stay within the frequency-consequence evaluation criteria derived from the TLRC and to implement risk management strategies to control the total integrated risk of the plant. Reliability targets for SSCs are determined based on the need to maintain each LBE within its LBE category (Anticipated Operational Occurrence, Design Basis Event, or Beyond Design Basis Event). RIPB SSC capability targets are defined in part by the selected design margins between the LBE frequencies and dose limits for that LBE category. Special treatment requirements for SSCs are derived to achieve the necessary and sufficient degree of reliability and capability of the SSCs. This is discussed in a companion report on the LMP SSC safety classification approach.

\section{Defense-in-Depth Adequacy}

The PRA models and supporting assumptions are based in part on the plant capabilities for DID reflected in the design, as well as assumptions about the limits placed on design and operation of the plant by assumed programmatic DID measures. Information developed in the PRA is used to help evaluate the SSCs responsible for preventing and mitigating accidents. The PRA also plays an important role in the identification of key sources of uncertainty, and this supports a feedback loop to identify possible enhancements to plant capability and programmatic aspects of DID. Hence, the PRA provides important input to the risk-informed evaluation of DID, complements the NRC's deterministic approach and traditional DID philosophy, and provides a more objective, RIPB means to systematically demonstrate DID adequacy and preservation. This is discussed in a companion report on the LMP approach to evaluating DID adequacy.

\section{LMP Final Report}

The LMP team produced a narrative report describing the processes, events, and documents involved in producing the ultimate Project deliverable product, NEI 18-04 "Risk-Informed Performance-Based Technology Inclusive Guidance for Non-Light Water Reactor Licensing Basis Development." This report contains a wealth of references to documents that future users of the LMP RIPB process may find useful. Tables within the report provide references to the 
NRC Agencywide Document Management System (ADAMS) Accession Numbers of many industry and NRC documents that future permit and license applicants may wish to reference in their own applications.

\section{LMP Lessons Learned, Best Practices, and Frequently Asked Questions and Responses}

The LMP team produced a report based on the experiences of early adopters of the LMP RIPB process which includes best practices, lessons learned, and frequently asked questions and responses. This report provides guidance to reactor designers on how to efficiently implement the LMP RIPB processes within their own organization and answers to 32 frequently asked questions from reactor designers.

\section{C.2 Frequently Asked Questions}

\section{Probabilistic Risk Assessment Frequently Asked Questions}

PRAQ1. How can the use of PRA technology to risk-inform the licensing of advanced nonLWRs be justified given the lack of operating experience with these reactors?

PRAQ2. How to develop adequate PRA data for initiating events and frequencies, component failure rates, maintenance unavailability, and other PRA data needs?

PRAQ3. What is the role of the PRA in the SSC safety classification process and how does safety classification influence the PRA models and data?

PRAQ4. What is the role of absolute and relative risk significance criteria in the LMP methodology?

PRAQ5. What is the applicability of 10 CFR 50 Appendix B to PRA in the LMP methodology?

PRAQ6. What is the available guidance for the systematic search for initiating events for the PRA on advanced non-LWRs?

PRAQ7. How does the LMP methodology identify and evaluate "cliff edge" effects?

PRAQ8. How does the structure of the PRA event tree logic impact the identification of the Required Safety Functions and the selection of the SR SSCs?

PRAQ9. How can the PRA standard requirements be met during the design stage when as-built and as-operated information is not available?

PRAQ10. What is the available guidance on how RSFs are determined, how they relate to FSFs?

PRAQ11. What guidance is available on the PRA treatment of safety functions provided via passive means and utilizing inherent reactor features?

PRAQ12. How can the LMP methodology be applied using dynamic PRA method? 
PRAQ13. How does LMP address events that are not modeled in the PRA?

\section{Licensing Basis Events Frequently Asked Questions}

LBEQ1. What is the available guidance for how to develop mechanistic source terms using the PRA and supporting deterministic processes?

LBEQ2. How is the safety classification and special treatment of SSCs influenced by the placement of LBEs as AOOs vs. DBEs or BDBE?

LBEQ3. Is there additional information available on the selection of the F-C Target anchor points for evaluating the risk-significance of LBEs?

LBEQ4. What insights were obtained for using the F-C charts from the LMP tabletop exercises and from discussions with the NRC Staff regarding DG-1353 and SECY-19-0117?

\section{SSC Classification Frequently Asked Questions}

SSCQ1. What guidance is available on how to select among candidates for SR SSCs and possible conflicts with ARDCs?

SSCQ2. What guidance is available for how to classify NSRST SSCs and how to come up with STs.

SSCQ3. What guidance is available for how to consider whether an SSC is classified as NSRST as necessary for adequate DID?

SSCQ4. What guidance is available for how to address the full scope of SSCs in a plant including I\&C, support systems, active SSCs, passive SSCs relying on inherent features, and SSCs necessary to implement safety significant operator actions?

SSCQ5. What guidance is available for how to consider the need to protect SR SSCs against DBEHLs and how to consider the requirements for NSR and NSRST SSCs?

SSCQ6. What guidance is available to discuss how SSC classification flows down from RSFs to major components and subcomponents to establish SRDC at the lowest level?

SSCQ7. What guidance is available on how to set reliability and capability targets for safety significant SSCs?

SSCQ8. What is the relationship between the Maintenance Rule scope and the LMP SSC approach to assuring reliability and capability targets for NSRST and NSR components?

SSCQ9. IEEE standards for I\&C design only consider two safety classifications, 1E or non-1E. $1 \mathrm{E}$ is for safety functions or supporting systems that perform safety functions. Software QA for $1 \mathrm{E}$ is very complex and expensive. $1 \mathrm{E} \mathrm{V} \& \mathrm{~V}$ is also complex and difficult (i.e. exploration for unintended functions and behavior). The same concept of existing industrial codes and standards having binary rules for safety-related and non-safety-related SSC, but not addressing the 
"middle" NSRST, is encountered often across standards development organizations. Should equipment classified by LMP as NSRST be treated as 1E or non-1E (or, as safety-related or nonsafety-related) and why?

\section{Defense-in-Depth Frequently Asked Questions}

DIDQ1. What guidance is available on how to examine the results, limitations, uncertainties, and omissions from the PRA for making IDP decisions that impact SSC safety classification and ST or deciding on practical compensatory actions?

DIDQ2. What guidance is available on how to organize the IDP and update the DID baseline through design iterations?

DIDQ3. What is the distinction between the IDP and the IDPP and why is it important?

DIDQ4. What additional guidance is there regarding the evaluation of Plant Capability DID for low dose or no dose (zero consequences) LBEs and the determination of NSRST SSCs?

\section{Project Management Frequently Asked Questions}

PMQ1. What guidance is available for how to manage the iterative process of design development, PRA development, and selection of codes and standards for SSCs?

PMQ2. How does a designer know that they are completely done implementing the LMP RIPB process with a reactor design? What is the definitive "pencils down" "finish line" event? 